\title{
LYMPHOCYTE SUBSETS AND MACROPHAGES IN THE MALE GENITAL TRACT IN HEALTH AND DISEASE \\ by
}

MOSTAFA IBRAHIM METWALLYY EL-DEMIRY

M.B. B.Ch., M.Sc. (Urol.)

Thesis presented for the degree of Doctor of Philosophy University of Edinburgh

1987

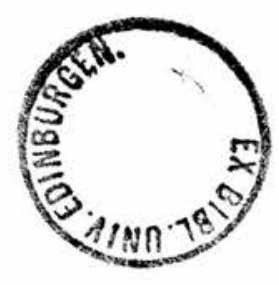




\section{THE UNIVERSITY of EDINBURGH}

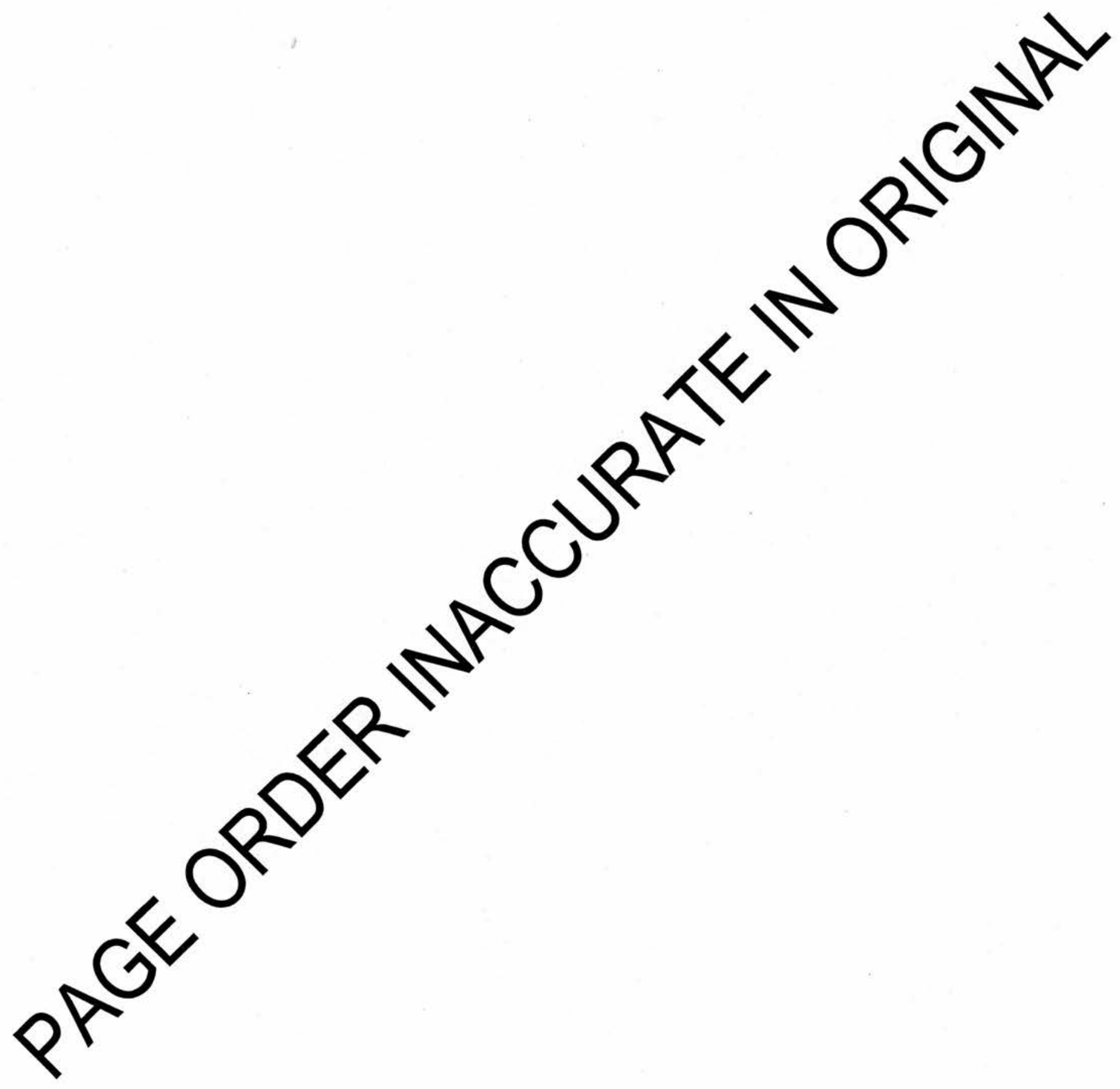




\section{LIST OF CONTENTS}

List of Figures

vii

List of Tables

$\mathrm{xi}$

Dedication

xiv

Declaration

$\mathrm{xv}$

Abstract

xvi

CHAPTER I: GENERAL INTRODUCTION

I.A Introduction

I.B Anatomical and Functional Aspects of the Male Reproductive Organs

I.B.1 The Testes

I.B.2 The Rete Testis

I.B.3 The Epididymis

I.B.4 The Ductus Deferens (Vas Deferens)

$\underline{\text { Page }}$

I.B.5 The Prostate

I.B.6 Seminal Vesicles

I.C Autoimmunity to Sperm

I.C.1 Historical Perspective

I.C.2 Antigens in Human Sperm and

I.C.3 The Immune Response to Sperm Antigens

and the Mechanism involved in

Immunological Infertility

I.C.3.1 Humoral immunity

I.C.3.2 Cell-mediated immunity 16

I.C. 4 Aetiology of Sperm Autoimmunity

I.C.5 Genital Tract Infection

I.D The Lymphoid System and Lymphocyte

Subpopulations

I.D.1 Introduction

I.D.2 The Organs of the Immune System

I.D.2.1 The thymus gland

I.D.2.2 The bursa of Fabricius and bursa equivalents

I.D.2.3 Spleen

I.D.2.4 Lymph node

I.D.2.5 Other secondary lymphoid tissues 
I.D.3 The Principal Cells of the Immune System

I.D.3.1 T-1ymphocytes

I.D.3.2 B-lymphocytes

27

I.D.3.3 Phagocytic cells

I.D.3.4 Antigen-presenting cells

IE Immunohistological identification of leucocyte Subpopulations

I.E.1 Introduction

I.E.2 Immunocytochemical Methods

I.E.3 Selection of the Immunohistological Staining Technique

I.E.4 Modes of Application of Immunoperoxidase Procedures

I.F Aims of the Study

CHAPTER II: MATERIALS AND METHODS

II.A Collection and Processing of Tissue Specimens

II.A.1 Normal Tissue Specimens 44

II.A.2 Clinical Specimens 44

II.A.2.1 Testicular biopsies from subfertile patients

II.A.2.2 Epididymal biopsies

II.A.2.4 Urinary bladder biopsy specimens

II.A.3 Tissue Preparation Techniques

II.A.3.1 Handling of tissue specimens 47

II.A.3.2 Cryostat sectioning 48

II.A.3.3 Fixation and storage of

frozen sections

II.B Collection of Semen Specimens 48

II.B.1 Patients 48

II.B.2 Semen 50

II.B.3 Microbiological Screening of

II.B.4 Processing of Semen Specimens 51

II.B.4.1 Introduction 51

II.B.4.2 Preparation of a single cell suspension of the ejaculate 52

II.B.4.3 Preparation of cytocentrifuge smears of washed ejaculate 53

II.B.4.4 Preparation of mononuclear and polymorph nuclear smears from human peripheral blood 
II.C Monoclonal Antibodies

II.C.1 Preparation and Storage of Monoclonal Antibodies

II.C.2 Titration of Antibody Concentration 59

II.C.3 Other Cytochemical Reagents

II.C.3.1 Peroxidase conjugate

60

II.C.3.2 3-3'diaminobenzidine

tetrahydrochloride

II.D Immunoperoxidase Staining of Tissue Sections and Cell Smears

II.D.1 Procedure

61

II.D.2 Specificity

II.D.3 Interpretation of Labelling Results

II.E Immunohistological Analysis and Scoring

II.E:1 Tissue Sections

II.E.2 Ejaculate Smears

II.E.3 Statistical Analysis

II.E.4 Photomicrography

CHAPTER III: OPTIMIZATION OF TECHNIQUES

III.A Routine Assessment of Monoclonal Antibodies 72

III.A.1 Technical Quality of Immunoperoxidase Stained Sections and Smears 72

III.A.1.1 Staining patterns 72

III.A.1.2 The optimum working dilutions of the antibodies

III.A.1.3 Specificity controls

III.A.1.4 Background staining

III.B Lymphocyte Subpopulations in Human Lymphoid

Tissues and Blood

III.B.1 Lymph Nodes and Tonsils 77

III.B.2 Spleen

81

III.B.3 Blood Leucocytes/Ejaculate Smears

III.C

Discussion

III.C.1 Technical Aspects

III.C.2 Lymphocyte Subpopulations in Lymphoid

III.C.3 Leucocytes/Ejaculate Smears

CHAPTER IV: LYMPHOCYTE SUBSETS AND MACROPHAGES IN THE TISSUES OF THE MALE REPRODUCTIVE TRACT

IV.A The Testis 
IV.A.2 Testicular Biopsies from Infertile Men 97

IV.B The Epididymis 105

IV.B.1 Normal Epididymal Tissues 105

IV.B.1.1 Histology 105

IV.B.1.2 T-Lymphocytes 105

IV.B.1.3 B-Lymphocytes 110

IV.B.1.4 Macrophages 110

IV.B.1.5 Anti-HLA-DR Reactive Cells 111

$\begin{array}{lll}\text { IV.B.2 Epididymal Biopsies from Patients } & \\ & \text { with Testicular Obstruction } & 114\end{array}$

IV.B.2.1 Histology 114

IV.B.2.2 Immunhistology 114

IV.C The Vas Deferens 121

IV.C.1 Lymphocytes 121

IV.C.1.1 The vasal part 121

IV.C.1.2 The ampulla of the vas 126

IV.C.2 Macrophages 126

IV.C.3 The Anti-HLA-DR Reactive Cells 126

IV.D The Seminal Vesicles 128

IV.D.1 Lymphocytes 128

IV.D.2 Macrophages 128

IV.D.3 The Anti-HLA-DR Reactive Cells 128

$\begin{array}{ll}\text { IV.E The Prostate } & 131\end{array}$

IV.E.1 Normal Prostatic Tissue 131

IV.E.1.1 Lymphocytes 131

IV.E.1.2 Macrophages 134

IV.E.1.3 Anti-HLA-DR reactive cells 134

$\begin{array}{lll}\text { IV.E.1.4 Anti-Leu } 7 \text { reactivity to } & \\ \text { normal prostatic tissues } & 134\end{array}$

IV.E.2 Benign Prostatic Hyperplasia Tissues 137

IV.E.2.1 Lymphocytes 137

IV.E.2.2 NK-cells 141

$\begin{array}{ll}\text { IV.E.2.3 } & \begin{array}{l}\text { Macrophages and anti-HLA-DR } \\ \text { reactive cells }\end{array} \\ & 141\end{array}$

IV.F T-Lymphocyte Subsets in the Male Genital Tract: Summary of Results

$\begin{array}{ll}\text { IV.G Discussion } & 150\end{array}$

IV.G.1 Lymphocytes 150

IV.G.2 Macrophages 158

IV.G.3 Patients with Testicular Obstruction 160 
CHAPTER V: LYMPHOCYTE SUBSETS IN UROTHELIUM

$\begin{array}{ll}\text { V.A Introduction } & 165\end{array}$

V.B Normal Urothelium 165

V.B.1 T-Lymphocytes 165

V.B.2 B-Lymphocytes 170

V.B.3 Monocytes and Macrophages 170

V.C Bladder Biopsies from Patients with Carcinoma

in Situ 172

V.C.1 T-Lymphocytes 172

V.C.2 B-Lymphocytes 172

V.C.3 Monocytes/Macrophages 172

V.C.4 Anti-HLA-Dr Reactive Cells 174

$\begin{array}{lll}\text { V.D Discussion } & 174\end{array}$

CHAPTER VI: IDENTIFYING LEUCOCYTES AND LEUCOCYTE SUBPOPULATIONS IN SEMEN USING MONOCLONAL ANTIBODY PROBES

VI.A Introduction

VI.B Immunoperoxidase Staining of Cytocentrifuged

Smears of Semen

VI.C Leucocytes in the Semen 179

V.C.1 Fertile Men 179

V.C.2 Patients with an Infertile Marriage 179

VI.D Microbiological Investigations 183

VI.E Relationship of Leucocytes to Bacterial Counts 185

VI.F Relationship of Leucocytes to Antisperm Antibodies

VI.G Relationship of Leucocytes to Sperm Density, Motility and Subsequent Fertility 188

$\begin{array}{lll}\text { VI.H Discussion } & 188\end{array}$

$\begin{array}{ll}\text { VI.I Conclusion } & 194\end{array}$ 
CHAPTER VII: CONCLUDING DISCUSSION

Acknowledgements

Bibliography

200

Appendices 


\section{LIST OF FIGURES}

Figure No.

Page

I.1 a) Immature germinal cells in the ejaculate 21

b) Leucocytes and epithelial cells in the ejaculate 21

I.2 Stages of T-cell differentiation in human beings 30

II.1 Exploratory scrototomy revealing unilateral epididymal obstruction

II.2 A Teddington type QR cryostat 49

II.3 A Shandon cytocentrifuge 55

II.4 A Car1 Zeiss Jena optic microscope fitted with an automatic photomicrographic camera ( $\mathrm{mF}$ AKS)

III.1 Immunoperoxidase staining of a cryostat section of 1ymph node for B-1ymphocytes using the monoclonal antibody anti-Leu 14

III.2 Immunoperoxidase staining of a cryostat section of tonsil for T-1ymphocytes using the monoclonal antibody Leu 4

III.3 Immunoperoxidase staining of a cryostat section of lymph node for helper/inducer T-cells using the monoclonal antibody Leu $3 a$

III.4 Immunoperoxidase staining of a cryostat section of tonsil for NK-cells using the monoclonal antibody Leu $11 b$

III.5 Immunoperoxidase staining of a cryostat section of spleen for T-cells using the monoclonal antibody Leu 4

III.6 Immunoperoxidase staining of a cytocentrifuge smear of human peripheral blood with the antihuman leucocyte monoclonal antibody

III.7 Immunoperoxidase staining of a cytocentrifuge smear of human ejaculate with the antihuman leucocyte monoclonal antibody

IV.1 Immunoperoxidase staining of cryostat sections of rete testis for (a) suppressor/cytotoxic T-cells and (b) helper/inducer T-cells using the monoclonal antibodies Leu $2 \mathrm{a}$ and Leu $3 \mathrm{a}$ 
IV.2 Immunoperoxidase staining of cryostat sections of normal testicular tissues with (a) Leu M3 and (b) 3.9 monoclonal antibodies which define monocytes/ macrophages

IV.3 HLA-DR positive cells in normal testicular tissue. (Cryostat section, immunoperoxidase, $\mathrm{x} 80$ )

IV.4 (a) Suppressor/cytotoxic T-cells (Leu $2 \mathrm{a}^{+}$) in a testicular biopsy from a patient with obstructive azoospermia; (b) helper/inducer T-cells (Leu $\mathrm{3a}^{+}$) in a testicular biopsy from a vasectomy reversal patient. (Cryostat sections, immunoperoxidase, $\mathrm{x}$ 160)

IV.5 T-lymphocyte subsets in patients with testicular obstruction

IV.6a Immune histological staining of a cryostat section of the body of the epididymis for T-1ymphocytes using the monoclonal antibody Leu 4.

IV.6b Suppressor/cytotoxic T-cells (Leu $2 \mathrm{a}^{+}$) within the epithelium of the head of the epididymis

IV.6c Helper/inducer T-cells (Leu $3 \mathrm{a}^{+}$) in the tail of the epididymis

IV.7 (a) Leu $\mathrm{M3}^{+}$cells (monocytes/macrophages) in normal epididymis; (b) the same field stained for T-1ymphocytes. (Cryostat sections, immunoperoxidase, $x$ 160)

IV.8 Immunoperoxidase staining of a cryostat section of the tail of the epididymis stained with the anti HLA-DR antibody

IV.9 T-1ymphocyte subsets in normal epididymis and epididymal biopsies from patients with testicular obstruction (mean counts per 100 epithelial cells)

IV.10 T-1ymphocyte subsets in normal epididymis and epididymal biopsies from patients with testicular obstruction (mean counts in lamina propria per cross section of epididymal duct)

IV.11 T-1ymphocyte subsets in normal epididymis and epididymal biopsies from patients with testicular obstruction (mean counts per HPF in interstitial tissue) 
IV.12 Immunoperoxidase staining of cryostat sections of epididymal biopsy from a vasectomy reversal patient stained with (a) Leu 4 antibody for all T-cells, and (b) Leu 3a antibody for helper/ inducer T-cells.

IV.13 Leu $\mathrm{M3}^{+}$cells (macrophages) in epididymal

biopsies from: (a) a vasectomy reversal patient;

(b) a unilaterally obstructed patient.

(Cryostat sections, immunoperoxidase, x 160)

IV.14 Immunoperoxidase staining of cryostat sections of vas deferens stained for (a) T-1ymphocytes and (b) HLA-DR antigens

IV.15 T-1ymphocyte subsets in normal vas deferens (mean counts per 100 epithelial cells)

IV.16 T-1ymphocyte subsets in normal vas deferens (mean counts in lamina propria per cross section)

IV.17 Immunoperoxidase staining of a cryostat section of seminal vesicles stained for suppressor/cytotoxic T-cells

IV.18 (a) Leu $4^{+}$cells (T-1ymphocytes) in normal prostate are seen in the lining epithelium, in the lumina of ductsand in the interstitium

(b) Leu $2 \mathrm{a}^{+}$cells (suppressor/cytotoxic

T-1ymphocytes) in the lining epithelium of prostatic acini. (Cryostat sections, immunoperoxidase, $\mathrm{x} 160$ and $x$ 320)

IV.19 Leu $\mathrm{M}^{+}$cells (macrophages) in normal prostate

IV.20 HLA-DR ${ }^{+}$cells in normal prostate

IV.21 Immunoperoxidase staining of a cryostat section of prostate tissue stained with Leu 7 antibody

IV.22 Immunoperoxidase staining of cryostat sections of $\mathrm{BPH}$ tissues stained for:

(a) suppressor/cytotoxic T-cells (Leu $2 \mathrm{a}^{+}$)

(b) helper/inducer T-cells (Leu $3 \mathrm{a}^{+}$)

IV.23 Immunoperoxidase staining of cryostat sections of prostatic tissue, obtained from a 54 year old patient with BPH and chronic prostatitis, stained for (a) activated T-cells $\left(1 \mathrm{~L} 2-\mathrm{r}^{+}\right.$) and (b) B-lymphocytes (Leu $14^{+}$) 
IV.24 T-1ymphocyte subsets in prostatic epithelium

IV.25 T-1ymphocyte subsets in the connective tissue stroma of the prostate

IV.26 Immunoperoxidase staining of cryostat sections of $\mathrm{BPH}$ tissue with Leu $11 \mathrm{~b}$ antibody

IV.27) T-1ymphocytes within the epithelium of the male

) genital tract

IV.28) T suppressor/cytotoxic cells within the epithelium

) of the male genital tract

IV.29) T helper/inducer cells within the epithelium of

) the male genital tract

IV.30) The ratio of $\mathrm{T}$ suppressor/cytotoxic to $\mathrm{T}$ helper/

) inducer cells within the epithelium of the male

) genital tract

V.1 $\mathrm{T}^{+}$(suppressor/cytotoxic T-1ymphocytes) within normal urothelium: a) ureter; b) urinary bladder

V.2 HLA-DR ${ }^{+}$cells within the epithelium and lamina propria of normal human ureter

V.3 Immunoperoxidase staining of cryostat sections of bladder biopsy obtained from a patient with carcinoma in situ stained with a) $\mathrm{T} 4$ and b) B1 monoclonal antibodies

VI.1 Immunoperoxidase staining of cytocentrifuge smears of semen stained with: a) antihuman leucocyte antibody for all leucocytes and b) Leu $2 \mathrm{a}$ antibody for suppressor/cytotoxic cells 


\section{LIST OF TABLES}

Table No.

$\underline{\text { Page }}$

I.1 Immunocytochemical methods

II.1 Source of male genital tract tissues used in these studies

II.2 Monoclonal antibodies to human leucocyte surface antigens used in these studies

III.1 Intensity of specific and background staining with the indirect immunoperoxidase technique using different dilutions of antihuman leucocyte monoclonal antibodies

III.2 Intensity of staining with indirect immunoperoxidase method using different dilutions of peroxidase conjugated rabbit-anti-mouse antibody

III.3 Percentages of 1ymphocyte subpopulations in normal peripheral blood (cytocentrifuge preparation of mononuclear cells)

IV.1 Reactivity of monoclonal antibodies specific for T-cells with rete testis - epithelium

IV.2 Reactivity of monoclonal antibodies specific for T-cells with rete testis - connective tissue

IV.3 Reactivity of monoclonal antibodies specific for T-cells with testicular biopsies from patients with obstructive azoospermia

IV.4 Reactivity of monoclonal antibodies specific for T-cells with testicular biopsies from postvasectomy patients

IV.5 Reactivity of monoclonal antibodies specific for T-cells with testicular biopsies from patients with unilateral testicular obstruction

IV.6 Mean (S.D.) 1ymphocyte counts per 100 epithelial cells within the epithelium of epididymis from 8 donors and 6 patients

IV.7 Mean (S.D.) 1ymphocyte counts per cross section of epididymal duct in lamina propria of epididymis from 8 donors and 6 patients 
IV. 8 Mean (S.D.) 1ymphocyte counts per HPF (x160) in interstitial tissue of epididymis from 8 donors and 6 patients

IV.9 T-1ymphocyte subsets in the vas deferens

IV.10 T-1ymphocyte subsets in the ampulla of the vas deferens

IV.11 T-lymphocytes subsets in the seminal vesicles

IV.12 (a) T-1ymphocyte subsets in normal prostatic epithelium

(b) T-lymphocyte subsets in the connective tissue stroma of normal prostate

IV.13a T-1ymphocyte subsets in the prostatic epithelium in BPH tissues

IV.13b T-1ymphocyte subsets in the connective tissue stroma of BPH tissues

IV.14 T-1ymphocyte subsets within the normal epithelium of the male genital tract

V.1 T-1ymphocyte subsets within the epithelium and lamina propria of normal human ureter

V.2 T-1ymphocyte subsets within the epithelium and lamina propria of normal human bladder

VI.la Leucocytes in semen detected by antihuman leucocyte (HLe-1) monoclonal antibody

VI.1b T-1ymphocytes in semen detected by anti-Leu 4 monoclonal antibody (a pan T-cell marker)

VI.2 Number of bacterial species isolated per semen sample

VI.3 Isolation rate of bacteria from 73 semen samples

VI.4 Quantitative bacteriology of most frequently isolated organisms

VI.5 Relationship between number of bacterial species per semen sample and leucocyte count

VI.6 Relationship between presence of anaerobes and leucocyte count 
VI.7 Relationship between ureaplasma culture and leucocyte count in infertile men

VI.8 Relationship between leucocyte counts and sperm antibodies

VI.9 Relationship between number of bacterial species per semen sample and sperm motility

VI.10a Relationship of total leucocyte counts to subsequent fertility

VI.10b Relationship of T-1ymphocytes to subsequent fertility 
To the Soul of my Mother,

To my Father, and

To my Wife

- xiv - 


\section{Declaration}

I declare that I have composed and written this thesis and that the work described in this thesis was entirely my own and performed by me unless otherwise acknowledged.

Mostafa I.M. E1-Demiry

April 1987 


\begin{abstract}
The ability of sperm cells to induce specific auto- and iso-immunity was reported as early as the turn of this century. However the mechanism controlling autoreactivity to sperm is not well known. As lymphocytes constitute the major cellular components of the immune system, determination of their anatomical location within the tissues of the male genital tract may be of considerable importance in understanding immunological infertility and other urogenital disorders. A series of monoclonal antibodies that react with human lymphoreticular cells was therefore used in an indirect immunoperoxidase technique to study their distribution throughout the male genital tract. The normal human tissues investigated were: testis, epididymis, vas deferens, prostate and seminal vesicles obtained from multi-organ transplant donors. The clinical specimens examined included surgical biopsies of testis, epididymis and prostate obtained during surgical procedures directed at the investigation and treatment of subfertile males and other patients.
\end{abstract}

All normal tissues, apart from the testis, were found to contain appreciable numbers of T-1ymphocytes (Leu $4^{+}$). T-cells of the suppressor/cytotoxic phenotype (Leu $2 \mathrm{a}^{+}$) were more abundant in the intraepithelial compartment while T-cells of the helper/inducer phenotype (Leu $3 \mathrm{a}^{+}$) were more common in the interstitial areas. With the exception of the prostate, very few B-cells were observed. Macrophages (Leu $\mathrm{M3}^{+}$) were identified within normal testicular tissues as well as the rest of the male genital tract. HLA-DR ${ }^{+}$ cells were also identified and the HLA-DR antigens were normally 
expressed on the lining epithelium of the rete testis, epididymis and vas deferens. Derangement of this pattern was observed in clinical specimens. Testicular biopsies from patients with testicular obstruction showed marked infiltration with 1ymphocytes mainly of the T-cell type. Biopsies from patients with benign prostatic hyperplasia showed increased infiltration with the helper/ inducer T-cells and other cell types such as natural killer cells (Leu $11 \mathrm{~b}^{+}$) and activated T-cells $\left(1 \mathrm{~L} 2-\mathrm{r}^{+}\right)$. These patterns of 1ymphoid cell distribution could provide an insight into both normal immunohomeostatic mechanisms and pathological events within the male genital tract.

The presence and distribution of 1ymphocyte subpopulations and macrophages within human urothelium in health and disease were also examined. T-1ymphocyte subsets and macrophages were identified in normal urothelium and shown to have a similar pattern of distribution to that seen in normal epithelium of the genital tract. The existence of these cell populations may contribute to the health and protection of urothelium, particularly in resistance to infection and tumour surveillance.

The presence of leucocytes and their subpopulations was also studied in the ejaculate from 69 men with an infertile marriage and 12 fertile men. Leucocytes were found in large numbers in the fertile men compared with the patients. Lymphocytes were found in $20 \%$ of the patients. There was no correlation between leucocyte counts and growth of microorganisms. These results cast doubt on the conventional criteria of subclinical genital tract infection, namely positive culture and excess leucocyte counts. 
CHAPTER I

GENERAL INTRODUCTION 


\section{I.A INTRODUCTION}

Fertility has been one of man's most desired attributes since the beginning of recorded history, and still remains of crucial importance to young couples today. Primary infertility has been estimated to affect $10 \%$ of married couples in the United Kingdom (Buxton and Southam, 1958). Furthermore, a male factor is present in, or contributes to, approximately $50 \%$ of the observed infertility problems (Ross, 1983). In spite of this problem, infertility is under-researched and current treatments are generally empirical.

Sperm antibodies are thought to account for some cases of infertility. Evidence for this is: 1) these antibodies are found more frequently in the infertile population when compared with a fertile one (Hargreave et al., 1980); 2) long-term follow-up of couples with sperm antibodies has shown that, when antibodies are detected above a certain level, the chance of fertility is very low (Rumke et al., 1974); 3) steroid treatment in some cases appears to result in fertility (Hendry et al., 1979, 1981).

The incidence of significant levels of sperm antibodies in the male partner of an infertile marriage has been reported to be approximately 9-10\% (Hendry et al., 1977; Hargreave et al., 1982a). This group of patients represents one of the few potentially treatable groups because treatments already exist for modulating immune responses. However, immunosuppression is a potentially dangerous treatment and our understanding of immune infertility is so limited that it is questionable if it is ethical to prescribe dangerous treatment in light of our current understanding. 
Considerably more data are needed before we can define clearly which patients require treatment and which treatment is to be preferred.

During the past few years our understanding of the complex cellular and molecular interactions involved in the immune responses has dramatically increased. This has been attributable in part to the development of monoclonal antibody probes (Kohler and Milstein, 1976) which have facilitated the identification, quantitation and isolation of both cellular and molecular components of the immune system. Of paramount importance in this respect have been those monoclonals which recognise lymphocyte subsets and other cells of the immune system. Several panels of monoclonal antibodies against surface antigens on $\mathrm{T}-$ and $\mathrm{B}-1 \mathrm{ymphocyte}$ subsets are commercially available. Such reagents are invaluable diagnostic tools for the immunophenotyping of lymphocytes and for the analysis of immune dysfunction. Monoclonal antibodies can determine the proportion and absolute numbers of $\mathrm{T}-$ cells and $\mathrm{T}$-cell subsets. The ratio of inducer/suppressor cells appears to be a particularly significant parameter since it provides a measure of the inter subpopulation balance within the immune system. The size of T-cell population and further division of these lymphocytes into the helper/inducer and suppressor/cytotoxic subsets have also been studied in many autoimmune diseases including rheumatoid arthritis, systemic lupus erythematosus, multiple sclerosis and thyroid diseases (Schlossman and Reinherz, 1984). From these studies has emerged a new conceptual framework for viewing autoimmune diseases.

Nevertheless, in spite of this progress, there are still large gaps in our overall understanding of the immune system, especially in certain areas of local immunity. Local modulation of 
the immune response is of vital importance in both male and female reproductive tracts. In the case of the male genital tract, there is an ever increasing incidence of clinical problems in which the functioning or otherwise of the local immune system could be of crucial importance. Included among these problems, in addition to immune infertility, are sexually transmitted diseases such as acquired immunodeficiency disease (AIDS), malignancies of the prostate, testis, and a variety of inflammatory conditions of undefined aetiology such as prostatitis.

Although the antigenicity of sperm was recognized as early as 1899 (Landsteiner, 1899; Metchnikoff, 1899), the mechanism controlling autoreactivity to sperm is not well understood. To date, most studies in humans on autoimmunity to sperm membrane antigens have dealt with humoral response to sperm antigens. Relatively little attention has been given to the possible role of cell-mediated immunity to sperm. This has been hampered by the lack of suitable techniques to trace the cellular markers with any precision. The advent and availability of monoclonal antibodies have prompted us to investigate in detail the presence in the male genital tract of the major cellular components of the immune system, namely lymphocytes and macrophages. These studies were embarked upon because it was believed they would shed light on both normal immune homeostatic mechanisms and the pathological events within the male reproductive tract. This may enable a better understanding of male immune infertility with the hope that such understanding may yield new approaches to patient management. 


\section{I.B ANATOMICAL AND FUNCTIONAL ASPECTS OF THE MALE REPRODUCTIVE ORGANS}

\section{I.B.1 The Testes}

The testes are the male reproductive organs. They have two functional components, seminiferous tubules and interstitial cells. Sperm production is the primary function of the seminiferous tubules. Interstitial cells, located between the seminiferous tubules are primarily responsible for hormone production.

\section{I.B.1.1 Seminiferous tubules}

The fibrous tunica propria (the tubular wall) enveloping the seminiferous tubule consists of several concentric layers: an inner acellular zone (basal lamina), an intermediate myoid layer and an outer adventitial region (Bustos-Obregon, 1976). Within the tubular wall, the seminiferous epithelium comprises two types of cells: Sertoli or supporting cells (Sertoli, 1865) and germ cells.

Sertoli cells: These form a continuous and complete lining within the tubular wall where they extend from the basal lamina to the lumen of the seminiferous tubules (Fawcett, 1975; Nistal et al., 1982; Schulze, 1984). Tight junctions and other components of junctional specialization between adjacent Sertoli cell membranes first arise during puberty with the development of the first germatocytes (Kormano, 1967; Vitale et al., 1973). These tight junctions are the morphological site of the blood-testis barrier (Koskimies et al., 1973; Setchell et al., 1980). They divide the 
germinal epithelium into a basal and an adluminal compartment in many species (Dym and Fawcett, 1970), including man (de Kretser and Burger, 1972). The basal compartment contains spermatogonia and early spermatocytes, whereas the adluminal compartment contains late spermatocytes and spermatids. By isolating these haploid germ cells within the adluminal compartment, the blood-testis barrier ensures that their specific antigens remains separated from the immune system and may provide protection during meiosis from circulating toxic substances.

Germ cells: Within the seminiferous tubules the germ cells occupy the space between the basal lamina and the lumen of the tubule. Morphological analysis (Clermont, 1963) revealed the presence of different types of germ cells in the human testis, including spermatogonia (types A and B), primary spermatocytes, secondary spermatocytes and spermatids.

The process of sperm production is called spermatogenesis which can be subdivided into three phases:

1) Spermatogonial proliferation and stem cell renewal (Clermont, 1972): the division of some type A spermatogonia gives rise to cells destined to become spermatocytes (B-spermatogonia), whereas other, apparently identical, cells give rise to more stem cells.

2) Meiosis: the type B spermatogonia divide mitotically to form the primary spermatocytes that will undergo meiosis (Kerr and de Kretser, 1981); a reductional division, followed by an equatorial division, resulting in the production of daughter cells with the haploid chromosome number: the round Sa spermatids. 
3) Spermiogenesis: during spermiogenesis, the round Sa spermatids metamorphose into mature spermatids and finally transform into highly organised spermatozoa.

Extensive changes occur in both the spermatid cytoplasm and nucleus (Kerr and de Kretser, 1981) and include formation of the acrosome, formation of the flagellum and loss of cytoplasm. Though partially visible by light microscopy, many of these changes can only be observed by electron microscopy (Holstein, 1976a).

The entire process of spermatogenesis takes approximately 70 days (Heller and Clermont, 1964), and it is not very efficient in that only one out of five of the mitotic offspring of the spermatogonia successfully matures to a spermatocyte (Huckins, 1978). This redundancy usually would be of small consequence, but excess sperm antigenic material that is potentially foreign to the host must be degraded and cleared in a manner that avoids immune stimulation of the host.

\section{I.B.1.2 Interstitial (intertubular) tissue}

The interstitial tissue between the seminiferous tubules contains Leydig cells (Leydig, 1850), fibroblasts, macrophages, occasional mast cells, blood vessels and nerves (Christensen, 1975; Connel1 and Conne11, 1977). 


\section{I.B.2 The Rete Testis}

The seminiferous tubules are long V-shaped tubules, both ends of which usually terminate in the rete testis (Roosen-Runge and Holstein, 1978). The rete testis extends along the longitudinal axis of the testis parallel to the axis of the epididymis and opens into the ductuli efferentes at the cranial pole of the testis. The ducts and channels of the rete testis are lined by squamous epithelium.

The rete testis is more permeable than the seminiferous tubule to the egress of sperm antigens or molecules and cells that are involved in immune processes (Haas and Beer, 1986). The concentration of sperm in the rete is only one-half that found in more distal segments of the male genital tract (Jones, 1977).

\section{I.B.3 The Epididymis}

In man, the ductuli efferentes - that arise from the rete testis - gradually coalesce in the distal region of the head (caput) of the epididymis to form the ductus epididymis (Holstein, 1976b). The ductus epididymis is a single highly convoluted duct which forms the remainder of the caput epididymis and all of the corpus and cauda epididymis. The caput epididymis (the head), which contains the ductuli efferentes and the initial part of the ductus epididymis, receiving spermatozoa and fluid from the testicular efferent ducts represents the principal zone of fluid resorption and the initiation of sperm maturation (Cooper, 1986). The corpus epididymis (the body) is the principal region for 
completion of sperm maturation and the cauda epididymis (the tail) functions as a region for sperm storage (Amann and Howards, 1980). Approximately 12 days are necessary for transport of sperm through the 12-15 foot long epididymis. During this time there are changes in sperm morphology, chemical composition, antigenicity and metabolism that are associated with increased fertility (Urry, 1981). De novo synthesis of surface components during epididymal passage has been detected recently using monoclonal antibodies (Eddy et al., 1985).

The epithelium lining the lumen through which the sperm cells pass consists of a variety of cell types, the main functions of which are the resorption and modification of testicular fluid entering the epididymis and the elaboration of epididymal secretions. Resorption of fluids increases the concentration of sperm cells and that of secreted compounds (Cooper, 1986). A simple columnar epithelium consisting mostly of ciliated and non ciliated principal cells lines the ductuli efferentes (Holstein, 1969). The epithelium of the ductus epididymis is of the prismatic pseudostratified type consisting of two major cell types: the principal cells and basal cells (Horstmann, 1962; Holstein, 1976b; Vendrely, 1981). Intraepithelial 1ymphocytes, originally described as halo cells (Reid and Cleland, 1957), are found throughout the epididymis. In man, they have recently been identified as T-1ymphocytes (Ritchie et al., 1984a).

The lamina propria enclosing the epithelium of the ductus epididymis consists of a basal lamina and an outer layer of connective tissue elements. 


\section{I.B.4 The Ductus Deferens (Vas Deferens)}

The ductus deferens is a distal extension of the ductus epididymis. It carries spermatozoa and seminal fluid from the cauda epididymis to the ejaculatory duct where it forms the excretory duct of the seminal vesicle. It plays an important role not on $1 y$ in sperm transport but a 1 so in the maintenance of epididymal sperm reserve. Ejaculated sperm are derived from the vas deferens and cauda epididymis in a $2 / 3$ ratio (Overstreet, 1986). Histologically, the ductus deferens is divided into two segments of very unequal length: the vasal part and the ampulla (Vendrely, 1981). The former is cylindrical in shape and its structure resembles that of the cauda epididymis. The latter is dilated and fusiform with a morphology similar to that of the seminal vesicle (Riva, 1982).

The wall of the vasal part is made up of a mucosa, three concentric layers of smooth muscles and an adventitia (Hoffer, 1976). The mucosa (the epithelium together with the underlying connective tissue of the lamina propria) is thrown up into a series of longitudinal folds, a feature which distinguishes the vas from the adjacent cauda epididymis. The epithelium within the mucosa is tall columnar pseudo-stratified epithelium with long stereocilia. According to Hoffer (1976), four types can be distinguished: principal cells, dark or pencil cells, mitochondrion rich cells and basal cells. 


\section{I.B.5 The Prostate}

The prostate is the largest accessory sex gland of the male. The gland is composed of alveoli that are lined with tall columnar secretory epithelial cells. The acini of these alveoli drain by a system of branching ducts mainly into the distal part of the prostatic urethra in close proximity to the orifices of the ejaculatory ducts (McNeal, 1981). This permits the secretions of the prostate to be added to other seminal plasma components arriving through the ejaculatory ducts, which bring spermatozoa and secretions from the testes, epididymis, ampulla and seminal vesicles. The prostatic fluid contributes more than $30 \%$ of the fluid volume of the ejaculate, with a normal volume of secretion of $0.5 \mathrm{ml}$. The bulk of the first portion of an ejaculate is derived from prostatic secretions, and the subsequent portion is derived from the seminal vesicles (Mann and Mann, 1981).

The alveoli and ducts of the prostate are embedded in a tissue matrix composed of stromal components, including fibromuscular, vascular and connective tissue. The prostatic epithelium is composed of three types of cells: the secretory (principal) epithelial cells, the basal cells, and/or the stem cells (Aumuller, 1983). 


\section{I.B.6 Seminal Vesicles}

The seminal vesicles in humans are two glands whose content is diversed in the terminal part of the ductus deferens, immediately above the ejaculatory ducts. The seminal vesicles were so named because it was erroneously believed that they store semen and sperm. Although their secretions contribute $46-80 \%$ of the ejaculate, with an average volume of $2.5 \mathrm{ml}$ (Mann and Mann, 1981), they do not store sperm or secretions made elsewhere. Fructose from the seminal vesicles is the major energy source for ejaculated sperm. In addition, the seminal vesicles provide a number of proteins which cause coagulation of the ejaculate (Jenkins et al., 1978). The gland consists of a villous mucosa (epithelium and lamina propria), a layer of smooth muscle and an external sheath of connective tissue (adventitia). The mucosa is thrown into a series of folds and contains pseudostratified columnar or simple columnar epithelium. Two main types of epithelial cells can be recognised: the principal secretory cells and the basal cells. 


\section{I.C AUTOIMMUNITY TO SPERM}

\section{I.C.1 Historical Perspective}

The history of sperm immunology goes back to the turn of the century when the immunogenicity of spermatozoa was demonstrated, first in heterologous systems independently by Landsteiner (1899) and Metchnikoff (1899) and shortly afterwards in a homologous system by Metlanikoff (1900). Similar studies were carried out by Von Moxter (1900) during the same period. Half a century passed before Rumke (1954) and Wilson (1954) reported male autoimmunity to sperm. Each author reported, independently of the other, on two patients who had remained childless and whose serum contained sperm agglutinating antibodies and thereby they stimulated intensive clinical research in this area. Nevertheless, the presence of sperm reactive antibodies in the serum of fertile members of many species raised the question of whether such antibodies could play a role in infertility. Experiments by Katsh (1959); Edwards (1964); McLaren (1964) and Bell and McLaren (1979) demonstrated that immunity to sperm induced by intraperitoneal inoculation of females with spermatozoa of the same or heterologous species impaired reproductive performance. In vitro exposure of spermatozoa to antisera also reduced their egg-penetrating ability (Menge, 1971; Seki and Mettler, 1982). Studies by Tung et al. (1980) and Yanagimachi et al. (1981) have shown that autoantibodies induced in male guinea pigs impair the acrosome reaction in vitro and prevent zona penetration by acrosome-reacted sperm. 
At the same time that the experimental basis of immunity to spermatozoa was first documented to cause induced infertility in animals, Rumke and Hellinger (1959) found that a significant number of men with infertility manifested an autoimmunity to spermatozoa. Subsequent1y, Franklin and Dukes (1964) also demonstrated sperm agglutinins in the sera of women whose failure to conceive was otherwise unexplained.

\section{I.C.2 Antigens in Human Sperm and Seminal P1asma}

Shulman (1975) has classified the antigens of the male reproductive tract into two systems. The first antigenic system includes the testis, epididymis and spermatozoa. The second is that of the prostate gland and other accessory glands of reproduction, namely seminal vesicles and bulbourethral glands. The semen represents a joining of the components of these two antigenic systems, and has been described as "an antigenic nightmare" (Jones, 1980).

Human seminal plasma and sperm have heterogeneous antigenic profiles (Shulman, 1975). Seminal plasma contains a vast array of antigens to which autoantibodies never seem to have been demonstrated (Hjort, 1983). This includes sperm-coating antigens which are seminal plasma components adsorbed so firmly to the surface of spermatozoa that they cannot be washed (e.g. ABO blood group determinants and lactoferrin). However, sperm-coating antigens have been shown to be potent immunogens in heterologous immunisation (Weil, 1960). 
Of great interest in terms of immunological infertility, however, are the intrinsic cell surface antigens of the spermatozoa itself. During the various stages of spermatogonial maturation, there are continual additions and deletions of spermatogenic cell surface antigens. It has been estimated that up to $50 \%$ of the antigens on mature spermatozoa were not present on spermatogonia (Romrel1 and O'Rand, 1978). The amount of sperm specific antigens exponentially increases from approximately $4 \%$ to greater than $96 \%$ by the late spermatid stage (Romrell and $0^{\prime}$ Rand, 1978). As a result of this evolution of sperm antigens, because of the changes that occur during the maturation process of spermatozoa, mature spermatozoa would not be recognised as self by the host.

With the use of the antibody localization technique, the spermatozoon has been shown to possess intrinsic antigens on the acrosome, mid-piece and tail, some of which may provoke immunological infertility (Hamerlynck, 1970). Three distinctive human autoantigens to which spontaneously occurring sperm antibodies are directed have been partially purified (D'Almeida et al., 1981). Monoclonal antibodies directed against specific plasma membrane antigens of rabbit, mouse and human spermatozoa have been shown to impair fertilization in vitro (Saling et al., 1983). Sperm membranes possess not only sperm specific but also HLA (Halim et al., 1982), HY and adsorbed antigens (Shulman, 1975).

Sperm enzymes also contribute to the antigenicity of sperm. Hyaluronidase, acrosin and lactic acid dehydrogenase - LDH-X (or LDH-C4) have been studied extensively, but of these only the last enzyme presents itself on the surface of intact spermatozoa (Erickson et al., 1975). 


\section{I.C.3 The Immune Response to Sperm Antigens and the Mechanism involved in Immunological Infertility}

The classic reaction of the body to invasion by a foreign immunogenic substance is by humoral (antibody) and cellular immune responses. The $\mathrm{B}-$ and T-1ymphocytes are required for the generation of immune responses. B-lymphocytes are responsible for the expression of the humoral immunity, the production of antibodies, while T-1ymphocytes are the main effector cells of cellmediated immune reactions.

\section{I.C.3.1 Humoral immunity}

Most studies in humans on autoimmunity to sperm membrane antigens have dealt with humoral immunity by detection of sperm agglutination, immobilisation or cytotoxicity. In male sera the antibodies causing these phenomena belong mainly to the IgG class (Fjallbrant, 1969; Boettcher et al., 1971; Husted, 1975). Antibodies in seminal plasma, bound to the surface of spermatozoa in the ejaculate, are predominantly of the IgA class (Jager et al., 1980). Several studies have reported that the majority of seminal plasma IgA has a molecular weight associated with the dimeric immunoglobulin molecule produced by mucosal surfaces elsewhere in the body (Uehling, 1971; Witkin et a1., 1981). However, it is thought that transudation of circulating antisperm antibodies occurs via the prostate or that the prostate gland is capable of mounting a 
local immune response following antigen stimulation (Ablin et al., 1972).

There are several mechanisms by which sperm antibodies could interfere with the reproductive process: (1) interference with sperm motility and penetration of cervical mucus (Rumke and Hellinger, 1959); (2) lysis of antibody-coated sperm by complement (Beer and Neaves, 1978); (3) interference with sperm capacitation (Beer and Neaves, 1978); (4) blocking of sperm-oocyte interaction (Haas et al., 1980) and (5) leucocyte phagocytosis of antibodyopsonised sperm (London et al., 1985).

\section{I.C.3.2 Ce11-mediated immunity}

It must be remembered that antisperm antibodies are only one aspect of the host's specific immune responsiveness. Relatively little has been done to study immune infertility possibly associated with other major components of specific immunity, cell-mediated immunity. A recent summary by Dondero (1982) updates the limited amount of information available on this subject. The role of cell-mediated immunity in human infertility is not clear, even though it has been well-documented in certain experimentally induced allergic orchitis states. Active immunization with autologous or homologous testicular antigen preparations in Freund's adjuvant of men with prostatic carcinoma resulted in induction of humoral and cellular immunity as well as aspermatogenesis in some cases (Mancini et a1., 1965). 
Because of the importance of T-lymphocytes and delayed hypersensitivity against sperm antigens in the animal model (Rumke, 1982), several investigators attempted to demonstrate cellular immunity against sperm antigens. Various techniques have been used and in several studies patients had indications of cell-mediated immunity to sperm more often than controls (Dondero et al., 1980). Studies with either lymphocyte transformation tests or leucocyte migration tests have provided evidence for the occurrence of cell-mediated immunity to sperm in $20-50 \%$ of men from infertile couples, vasectomised men or men with various testicular disorders, whereas few normal men revealed positive reactions (E1-Alfi and Bassili, 1970; Nagarkatti and Rao, 1976; Thestrup-Pedersen et al., 1976; Dondero et a1., 1980).

Distinct alterations in the cells of the immune system were recently observed in infertile males and females with cytotoxic and/or haemagglutinating sperm antibodies in contrast to fertile and infertile subjects without sperm antibodies. Subsets of circulating lymphocytes from 20 fertile and 135 infertile couples were defined using monoclonal antibodies. The number of $\mathrm{T}$-helper/inducer cells as well as T-helper/T-suppressor ratio were significantly decreased, while B-1ymphocytes were increased in infertile males and females with sperm antibodies as contrasted with the control fertile and infertile groups without sperm antibodies (Mathur, 1985)

Nevertheless, autoimmunity at the level of the testis and/or the tissues of the reproductive tract will probably have to come from immunohistological studies of testis biopsies (Rumke, 1982). 


\section{I.C.4 Aetiology of Sperm Autoimmunity}

Several factors are probably responsible for the initiation of autoimmunity against spermatozoa. It is commonly accepted that the development of male autoimmunity is due to a breakdown in the blood-testis barrier, exposing the hitherto sequestered spermatozoal antigens to the general immune system. Conditions that result in a major disruption of the blood-testis barrier are associated with the formation of sperm antibodies. Although vasectomy is the most common cause of antigen leakage (Alexander and Anderson, 1979), other injuries, such as vas obstruction (Hendry et a1., 1982), testicular trauma (Haensch, 1973), torsion (Mastrogiacomo et al., 1982) biopsy study (Hjort et al., 1974) and malignancy (Guazziero et al., 1985), all have been associated with an increase in the incidence of sperm antibodies.

Recently, it has been reported that the subjects with immunity to sperm may genetically be predisposed to autoimmunity and have immune alterations consistent with their altered immunity. HLA-B7 and B8 antigens occurred together at a significant 1 y increased frequency in infertile males with sperm antibodies as contrasted with the sperm antibody negative control groups (Mathur, 1985).

Several studies have suggested an association between sperm antibodies and epididymitis (Fjallbrant, 1965), prostate vesiculitis (Fjallbrant and Obrant, 1968; Quesada et a1., 1968), and non-specific urethritis (Witkin and Toth, 1983). Genital tract infection might, through the creation of local inflammation, allow 
access of immune competent cells to sperm antigens to which they are not tolerant. However, the whole matter of genital tract infection would appear to be less clinically relevant than was formerly believed. In 1981 Fowler reviewed the literature and found little evidence to support infection as a frequent cause of infertility.

\section{I.C.5 Genital Tract Infection}

Male genital tract infection may be difficult to diagnose due to the paucity of symptoms and the lack of clear clinical, bacteriological, and seminal fluid criteria.

Some studies have shown no difference in the results of culture for microorganisms from semen samples from fertile and infertile populations and no correlation between sperm measurements (motility and density) and bacteriology. Comparing semen characteristics of fertile and infertile groups, McGowan et al. (1981) found that infection in the semen did not significantly affect the count, motility or volume of the specimen. Comhaire et al. (1980) and Naessens et a1. (1986) also found no significant difference in the semen motility and density of infertile males with and without infection. The mere presence of bacteria in the seminal plasma is an inadequate criterion for the diagnosis of infection (Comhaire et al., 1980).

Others believe that there is a substantial proportion of infertile men with very minor symptoms but significant infection. Eliasson et al. (1967) found that approximately $40 \%$ of infertile men in Sweden had cytological or bacteriological findings indicating 
infection or inflammation of the accessory genital glands. They suggested that asymptomatic genital infection may be a major cause of infertility (Eliasson and Johannisson, 1978). Quesada et al. (1968), Moberg et al. (1980) and Swenson et al. (1980) also reported that infection of the semen may reduce sperm motility and density and hence affect fertility.

There are two important reasons for this controversy. The first is a lack of agreement about criteria to diagnose subclinical genital tract infection and the second is that in many studies there has been a failure to culture the complete range of pathogenic microorganisms; in most studies chlamydia and ureaplasma have been omitted.

Traditional diagnosis is made by finding an excess of leucocytes in the ejaculate. However, the importance and upper limit of normality of white cells in the semen as well as the prostate vesicular fluid has not been clearly established (Morton, 1968; Johannisson and Eliasson, 1978). Also, several types of immature germ cells are found in semen including spermatids, spermatocytes and even spermatogonia (Holstein, 1983), and these sperm precursors may be confused with white blood cells when ordinary staining techniques are used. These early non separated spermatids that share a common cytoplasm may be mistaken for PMN-leucocytes, while round spermatids (Sa-b) and occasionally spermatocytes may be mistaken for 1ymphocytes (Fig. I.1). Because of these problems new techniques have been developed to facilitate discrimination between white blood cells and immature germ cells. For example, Endtz (1972) has applied a histochemical technique based on the use of benzidine for staining leucocytes while Nahoum 

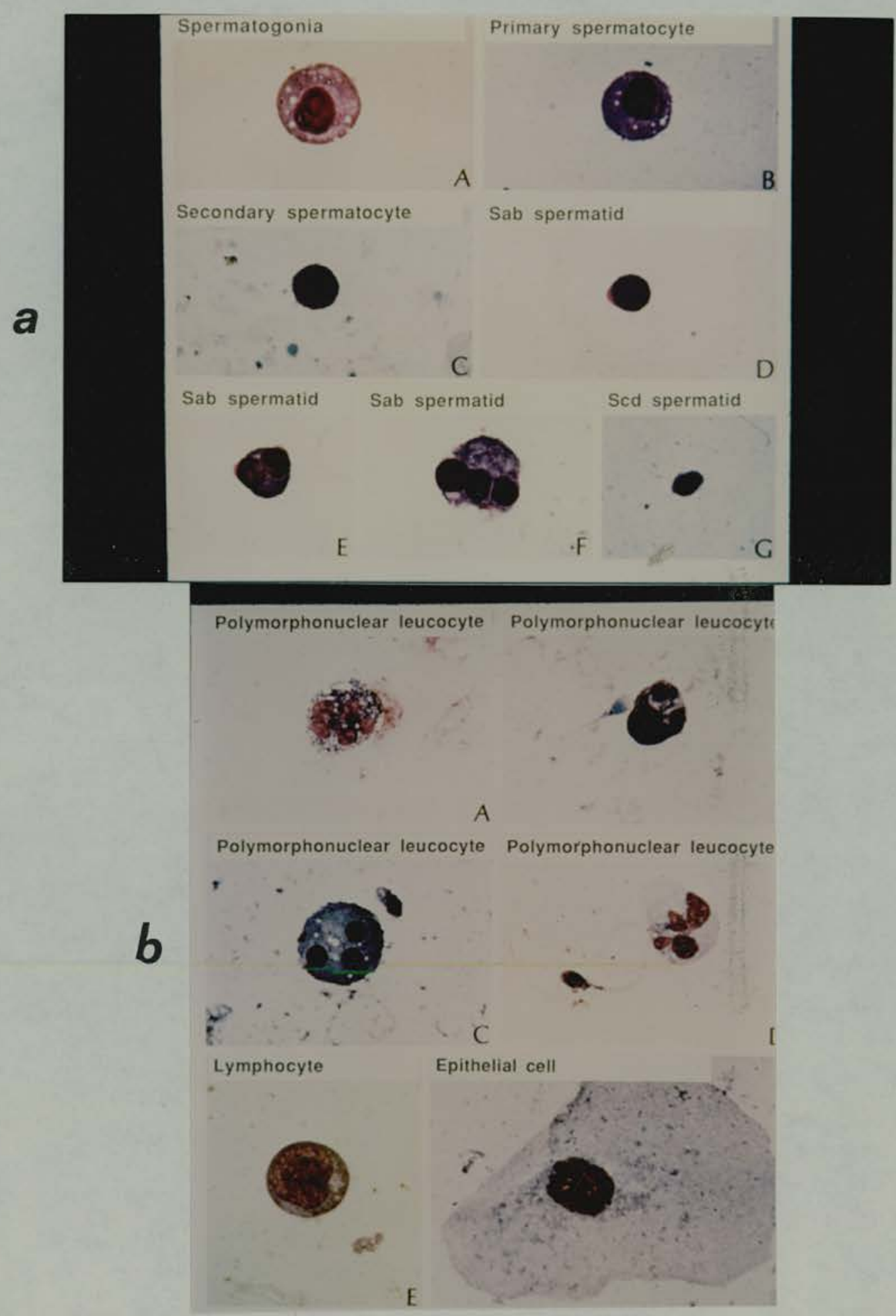

Figure I.1: a) Immature germinal cells in the ejaculate

b) Leucocytes and epithelial cells in the ejaculate (Source: Belsey et al., 1980) 
and Cardazo (1980) have proposed the use of 0-toluidine as a substitute for benzidine to detect these cells. Couture et al. (1976) have used a combination of Bryan's sperm stain (Bryan, 1970), which reacts with the acrosomal cap of the spermatozoa and of the spermatids, and Leishman's blood stain which interacts with leucocytes (Lillie, 1965).

These techniques, which depend mainly on the endogenous peroxidase activity of leucocytes, have certain limitations. Lymphocytes do not show peroxidase activity at any stage of maturation; basophils do not stain; and monocytes show faint diffuse cytoplasmic staining (Nelson and Davey, 1977). Furthermore, PMN leucocyte peroxidase activity depends on the age and condition of the cell and on the freshness of the smear at the time of staining (Belsey et al., 1980). Thus older PMN leucocytes and old smears may not exhibit this granular staining. Peroxidase activity also is unstable in light. In addition, peroxidase reaction may be negative in the neutrophils which appear in the peripheral blood in response to severe infection (Nelson and Davey, 1977), and this may also occur in the semen.

The attempt of Couture et al. (1976) to differentiate 1ymphocytes from Sa-b spermatids using Bryan's sperm stain (Bryan, 1970) for the acrosomal cap is also unreliable because the acrosome may not be fully developed and is difficult to demonstrate by light microscopy (Clermont, 1970). Also in abnormal semen there are many aberrations, and the number of immature spermatids with loosened or missing acrosomes may increase.

In view of the above a new accurate technique for precise identification of leucocytes in the genital tract secretions is needed before we can define clearly the role of genital tract 
infection in male infertility. Obviously the technique must also be able to differentiate leucocytes from immature germ cells.

\section{I.D. THE LYMPHOID SYSTEM AND LYMPHOCYTE SUBPOPULATIONS}

\section{I.D.1 Introduction}

The lymphoid system is composed of tissues and organs in which lymphocyte precursors and their derivatives originate, mature and localize. The various organs which comprise the lymphoid system may be classified as central or peripheral lymphoid organs.

The primary lymphoid tissues are the sites where lymphocytes are produced. Lymphocytes are derived from pluripotent haemopoietic stem cells that give rise to all other blood cells. The earliest blood-forming cells appear first in the yolk sac, later in the foetal liver and spleen, and finally in the bursa of Fabricius and the bone marrow, In an adult mammal, the bone marrow is the major source of haemopoietic stem cells; in birds, stem cells are found in the bone marrow and in the bursa of Fabricius. Lymphocytes differentiate from lymphoid stem cells, proliferate and mature into functional effector cells. In mammals, T-1ymphocytes are produced in the thymus gland from T-cell precursors while B-1ymphocyte precursors are believed to mature into B-cells in both the foetal liver and bone marrow. In birds, there is a specialized site of B-cell generation - the bursa of Fabricius. 


\section{I.D.2 The Organs of the Immune System *}

\section{I.D.2.1 The thymus gland}

On leaving the bone marrow and/or the foetal liver some of the progeny of lymphoid stem cells migrate to the thymus gland, where they undergo further differentiation and at this site are referred to as thymocytes. The human thymus consists of two lobes and each lobe is divided into small lobules. Each lobule, which is made up of a meshwork of epithelial cells packed with thymocytes, is arranged into an outer cortex and an inner medulla. The cortex is tightly packed with the majority of the relatively immature proliferating thymocytes whilst the medulla contains more mature thymocytes. Epithelial cells are more tightly aggregated in the medulla than in the cortex with superimposed interdigitating cells. Thymic epithelium produces several thymic hormones that cause T-cell maturation. Some of the epithelial cells, in the medulla, are arranged concentrically into thymic, Hassall's corpuscles. The lymphocytes that finally are released from the thymus into the circulation are referred to as thymus-derived or T-1ymphocytes (T-cells).

Within the thymus the T-1ymphocyte subsets (helper T-cells and cytotoxic T-cells) display a complex type of antigenic specificity in which they co-recognise a foreign antigen and an

The discussion in this section is based on reviews by Klein (1982); Holborow and Papamichail (1983); and Butcher and Weissman (1984). 
autologous membrane protein, coded for the major histocompatibility complex (MHC).

\section{I.D.2.2 The bursa of Fabricius and bursa equivalents}

The bursa of Fabricius, which is present in birds, is a lymphoepithelial organ located near the cloaca. Histologically, the bursal lymphoid follicles, within the lining epithelium, are organised into peripheral cortex and central medulla. Within the bursal follicles, the lymphocyte precursors transform into mature cells that leave the bursa as bursa-derived or B-lymphocytes (B-cells).

Mammals have no bursa, and the mammalian bursa equivalent may be located multifocally in various organs largely within the lymphoid tissue of the gastrointestinal tract.

Soon after $\mathrm{T}-$ and B-1ymphocytes are formed in the central 1ymphoid organs, they enter the circulation and migrate to secondary lymphoid tissues such as spleen, lymph nodes, and other peripheral lymphoid tissues. In these organs they localize, as described below, in a specific manner.

\section{I.D.2.3 Spleen}

Histologically, the tissue of the spleen is organised into red pulp and white pulp. Within the red pulp there are thin-walled blood vessels, the splenic sinusoids and the splenic cords. Within the white pulp there is the lymphoid tissue, arranged around a central arteriole, which comprises the periarteriolar lymphoid 
sheath (PALS) and the lymphoid follicles. Secondary follicles contain "mantles" and "germinal centres". The area between the white and red pulp is referred to as the marginal zone. The follicles contain mostly B-lymphocytes and the PALS mostly T-lymphocytes. Secondary follicles contain dendritic reticular cells in their germinal centre. The red pulp and marginal area contain plasma cells as well as macrophages.

\section{I.D.2.4 Lymph node}

Lymph nodes are small organs composed of reticular stroma and 1ymphoid parenchyma. Each node is loosely enclosed in a capsule. Beneath the capsule there is a subcapsular lymph space (sinus) into which the different lymphatics empty. The tissue of a lymph node is organised into an outer cortex and an inner medulla. The cortex is separated from the medulla by a paracortical region. The cortex contains more lymphocytes than the medulla, many of them aggregated into follicles either primary or secondary with germinal centres and mantle zones. The lymphocytes in the follicles are mainly B-cells and the germinal centres are the site of B-cell proliferation and differentiation. Most lymphocytes in the paracortical and interfollicular regions are T-cells. Within the medulla, there are medullary cords, separated by large sinuses, containing the majority of the plasma cells in the lymph node. 


\section{I.D.2.5 Other secondary 1ymphoid tissues}

In addition to the highly organised lymphoid organs, such as the lymph nodes and the spleen, the body contains other types of lymphoid tissue. This tissue can either be diffuse or organised into follicles. For example, dispersed aggregates of nonencapsulated lymphoid tissue are frequently found in the subepithelial connective tissue layer (lamina propria) of the gastrointestinal and respiratory tract. This mucosal associated lymphoid tissue is involved in the local immunity at mucosal surfaces.

The aggregated or solitary lymphoid follicles include the tonsils and the peyer's patches of the small intestine and the vermiform appendix of the large intestine.

\section{I.D.3 The Principal Cells of the Immune System}

\section{I.D.3.1 T-1ymphocytes}

A thymic microenvironment is necessary for the differentiation of T-1ymphocytes in all species. It appears that precursor bone marrow cells, prothymocytes, migrate to the thymus gland where they are processed, become functionally competent and are then exported into the peripheral lymphoid compartment which includes spleen, lymph and blood (Moore and Owen, 1967; Owen and Ritter, 1969; Owen and Raff, 1970). Moreover, profound changes in cell surface antigens accompany the various stages of T-cell 
ontogeny (Konda et al., 1973). Although initially identified by the presence of sheep erythrocyte binding receptors, it is not appreciable that leucocytes other than T-1ymphocytes may possess this receptor (West et al., 1978). The ability to define cell surface antigens that appear at specific stages of T-cell differentiation has allowed for the orderly dissection of T-cells into subclasses.

The most effective technique for identifying and separating subpopulations of peripheral T-cells are those employing specific antibodies to lymphocyte surface antigens. Thus, using such reagents, Cantor and Boyse (1975) were able to show that the mouse peripheral T-cell pool contains at least three separate T-cell sets. These are referred to as the Ly123 set, Ly1 set and Ly23 set, and they account for 50,30 and $10 \%$ respectively of the peripheral T-cell pool. Functional studies revealed that cells of the Lyl set are genetically programmed to induce (help) other cell types to divide and/or differentiate. Thus they induce B-cells to secrete antibody and stimulate monocytes, mast cells and precursor of T-killer cells to participate in cell-mediated immune responses. In contrast, cells of the L23 set are genetically programmed to kill or suppress other cells (Cantor and Boyse, 1975).

Functional subsets of human $\mathrm{T}$-cells were subsequently defined on the basis of the cell surface expression of Fc receptors for either IgM ( $T_{m}$ cells) or $I g G\left(T_{g}\right.$ cells). $T_{m}$ cells induced human polyclonal driven B-cell differentiation in vitro, whereas $\mathrm{T}_{\mathrm{g}}$ cells actively suppressed Ig synthesis (Moretta et al., 1977). However, these T-cell surface Fc receptors were subsequently shown 
to be unstable (Pichler et al., 1978) and are not as T-cell specific as originally believed.

Recently, using the technique of Kohler and Milstein (1976), monoclonal antibodies reactive with a variety of human 1ymphocyte cell surface differentiation antigens have been produced which enable the identification of a number of human lymphocyte subsets (Reinherz and Schlossman, 1980a). The cellular expression of the antigens defined by these monoclonal antibodies is summarised in Fig. I.2 and discussed briefly in the following paragraphs.

Unlike the majority of the thymocyte population which lack T1 and $\mathrm{T} 3$ antigens, all circulating peripheral $\mathrm{T}$-cells are $\mathrm{T}^{+}$and $\mathrm{T}^{+}$ (Reinherz et al., 1980a). This mature population could be further divided into distinct subsets of cells: $\mathrm{T}^{+}$and $\mathrm{T}^{+}$subsets. $\mathrm{T} 4$ antigen is expressed on approximately $60 \%$ of peripheral T-cells while the T5 antigen is present on $20 \%$ of peripheral T-cells. These two subsets correspond to the previously defined TH2- helper and $\mathrm{TH}_{2}{ }^{+}$suppressor cells respectively (Evans et al., 1978).

The T4 positive subset: It has been postulated that $\mathrm{T}_{4}^{+}$cells are necessary for the production of lymphokines in response to antigen (Meuer et al., 1982a), for the induction of B-cells to proliferate and differentiate into immunoglobulin-containing cells (Reinherz et al., 1980b), for the induction of precursor cytotoxic T-cells to become cytotoxic (Friedman et al., 1981), and for the generation of suppressor T-cells (Yachie et al., 1982). It has also been demonstrated that, with the appropriate target cells, a subset of $\mathrm{T}^{+}$lymphocytes may be cytotoxic as well (Moretta et al., 1981). 


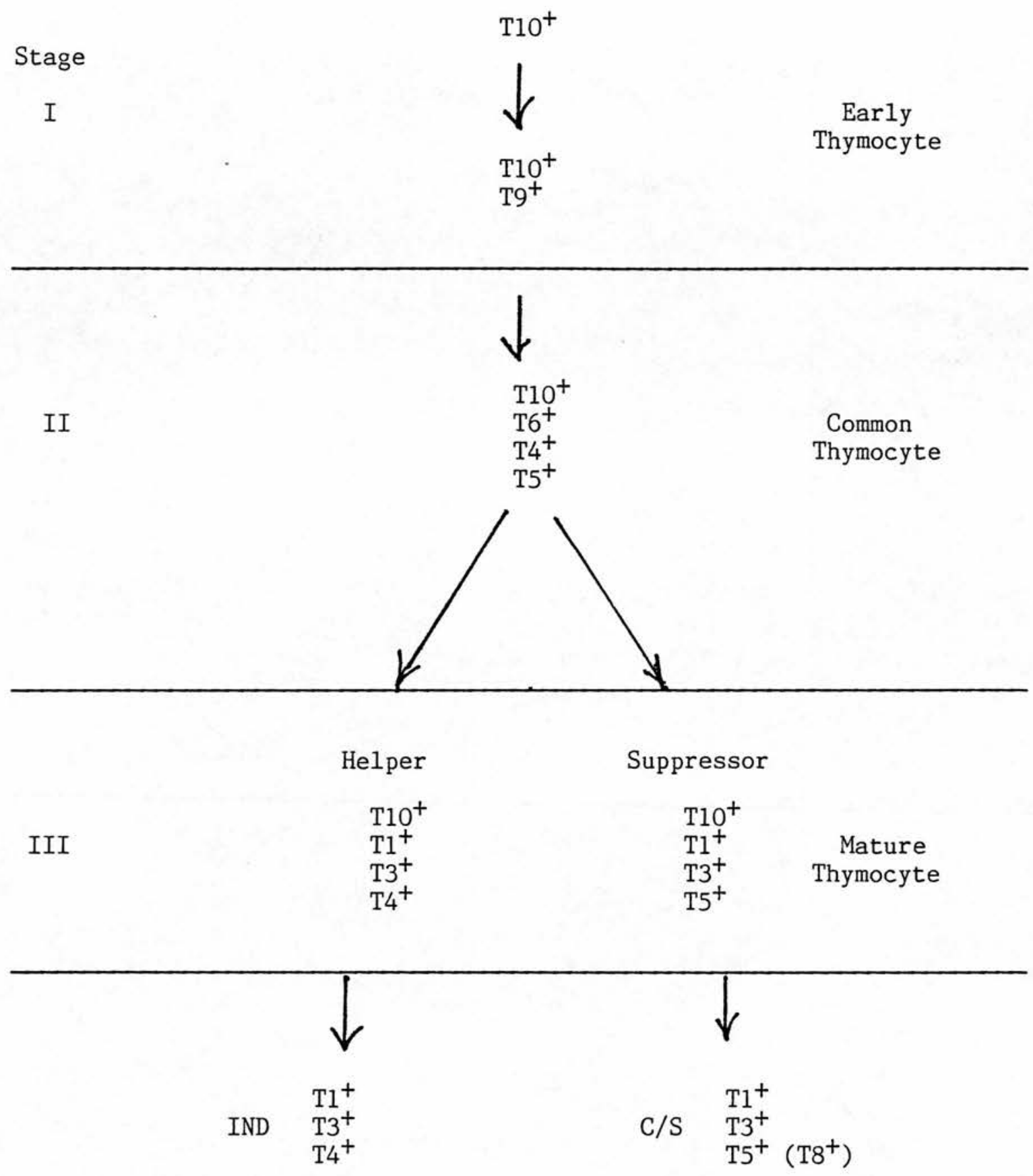

Figure I.2: Stages of T-cell differentiation in human beings.

Three discrete stages of thymic differentiation can be defined on the basis of reactivity with monoclonal antibodies. The most mature thymocyte population (Stage III) gives rise to the peripheral T-cell inducer (IND) and cytotoxic/suppressor (C/S) subsets. The cell surface antigens expressed during $\mathrm{T}$-cell ontogeny are shown (Reinherz et al., 1980a). 
Furthermore, within the $\mathrm{T}^{+}$population, cells with suppressor function have been identified (Thomas et al., 1981).

The $\mathrm{T} 5$ ( $\mathrm{T} 8$ ) positive subset: $\mathrm{The} \mathrm{T}^{+}\left(\mathrm{T} 8^{+}\right) \mathrm{T}-$ cell subset contains a mature population of cells with both suppressor and cytotoxic functions. It has been shown that the $\mathrm{T}^{+}$suppressed $\mathrm{B}-\mathrm{ce} 11$ immunoglobulin production (Reinherz et al., 1980b) and also suppressed autologous T-cell proliferative responses to alloantigens following activation with Conc. A (Reinherz and Schlossman, 1979).

\section{I.D.3.1.1 Subsets of human T-cells: correlation with}

\section{functional abilities}

The two major functionally defined subsets of T-cells, i.e. the helper/inducer and the suppressor/ cytotoxic subsets were identified by the T4 (Leu 3) and T8 (Leu 2) monoclonal antibodies, respectively (Reinherz et a1., 1979; Reinherz et a1., 1980c; Engleman et a1., 1981). However, this initial subdivision has turned out not to be absolute, but rather should be considered a useful generalisation. For example, cells with cytotoxic function have now been found within both the Leu 2 (T5/T8) and Leu 3 (T4) subsets (Moretta et al., 1981). A further distinction has been made between these subsets. Cytotoxic cells directed against Class I (HLA-A, B,C) MHC express Leu 2 while cytotoxic cells against Class II (HLA-DR) MHC express Leu 3 (Heuer et al., 1982; Meuer et al., 1982b). In addition, Leu $3 / T 4$ antigen expression is not exclusively restricted to T-1ymphocytes in that it is present in low density on human monocytes (Wood et al., 1983). Similarly, Leu 2 
is present on a subset of human natural killer (NK) cells, which do not express pan T-cell antigens such as Leu 4 or T3 (Lanier et al., 1983; Perussia et al., 1983). However these cells do not show MHC restriction in their cytotoxic function.

Attempts to generate single monoclonal antibodies which discriminate between cytotoxic versus suppressor cells, or monoclonals which demonstrate an absolute correlation with functional ability have not as yet succeeded. Hence, the most precise identification of a cell subset is not by the presence of a single antigen but rather by the correlated presence or absence of a number of different antigens.

\section{I.D.3.1.2 T-cell activation}

T-cell activation, which is an essential step in the initiation of the immune response, is induced only by antigen presented by an accessory cell that displays the Class II molecules (Ia-DR antigens) (Benacerrof, 1981). The macrophage or the antigen presenting cell must bind, process and present antigen in an immunogenic form to initiate an antigen-specific, T-cell dependent immune response (Ziegler and Unanue, 1981). At the same time, interleukin-1 (IL-1), an antigen-non specific macrophage-derived T-cell activating peptide, is synthesized and appears to induce the synthesis and secretion in turn of another T-cell lymphokine, interleukin-2 (IL-2) (Smith et al., 1980; Mizel, 1982). IL-2 is thought to be a primary stimulus for T-cell production of IFN-Y (Kasahara et al., 1983). The products of antigen-stimulated T-cells also appear to induce Ia expression by previously Ia-negative macrophages (Scher et al., 1982). There is evidence 
that IFN-Y may also induce macrophage Ia expression (Basham and Merican, 1983), the appearance of IL-2 receptors on T-cells as well as the secretion of IL-1 by monocytes (Steeg et al., 1982).

\section{I.D.3.2 B-Lymphocytes}

Morphologically, B-cells look the same as T-cells. However they can be distinguished by various markers. The most distinctive and useful marker for B-cells is their surface immunoglobulin (SIg). This surface immunoglobulin is present on all mature B-cells and is the receptor for specific antigen. B-lymphocyte precursors (pre-B) contain cytoplasmic IgM but do not express it on their surface (Osmond, 1980) while the most immature B-cells express surface IgM molecules (Cooper et al., 1980). With further maturation, B-cells begin to express surface IgD or multiple Ig classes and other membrane-bound glycoproteins, such as receptors for complement components and Fc portion of secreted IgG antibodies. The majority of B-cells express SIgM and SIgD. Small numbers express one of the other heavy chain types such as IgG and IgA. Within each B-cell class, some B-cells switch from the expression of $\operatorname{IgM}$ (and $\operatorname{IgD}$ ) to the expression of an IgG, IgA, or IgE immunoglobulin isotype. The B-1ymphocytes undergoing a heavy chain isotype switch may simultaneously express as many as three different immunoglobulin isotypes, e.g. IgM, IgD, and IgA (Abney et al., 1978).

In contrast to the variable heavy chain class of the SIg, each B-cell and all its progeny express only one light chain class and only one immunoglobulin variable region antigen-binding site 
(idiotype) and these are considered to be "clonal" markers for B-cells. Of the two, idiotype is much more specific but at present much more difficult to identify.

One of the most important surface components expressed by B-cells is the so-called Ia or DR antigens (mouse and human respectively). In mice, Ia determinants are acquired during B-cell maturation (Kearney et a1., 1977), whereas in man the equivalent HLA-DR molecules are expressed on both pre-B and B-cells (Kamps and Cooper, 1982).

The mature B-1ymphocytes, when activated to divide by antigenic stimulation and T-cell help, enlarge into blasts, divide and then transform back into small lymphocytes or the memory B-cells. These memory B-cells are relatively long-lived and more easily triggered on subsequent encounters with the same antigen than are virgin B-cells. Along with their T-cell counterparts, memory B-cells are responsible for the prompt, heightened antibody responses that result from secondary exposure to an antigen.

The final step in B-cell differentiation is to the mature plasma cells, immunoglobulin secreting cells. The progression from activated B-lymphocyte is accompanied by a gradual loss of surface immunoglobulin molecules, with the conversion from synthesis of membrane-type to secretory immunoglobulin molecules.

\section{I.D.3.3 Phagocytic cells}

The phagocytes of the body consist of two specialised groups of cells: the myeloid cells (neutrophils), and cells of the mononuclear phagocyte system (blood monocytes and tissue 
macrophages). The main function of neutrophils is to kill bacteria and other infectious agents by phagocytosis. The mononuclear phagocyte system has two main functions, performed by two different types of cells (Roitt et al., 1985): phagocytic macrophages whose predominant role is to remove particulate antigens, and antigen-presenting cells (APC) whose role is to present antigen to specific antigen-sensitive 1ymphocytes.

\section{I.D.3.4 Antigen-presenting cells}

A11 of the macrophage/monocyte lineage represent an important cell population in lymphocyte interactions in the immune response. The current consensus of opinion is that there are two lineages of macrophage/dendritic cells which are responsible for antigen presentation to lymphocytes (Friedman et al., 1981; Unanue, 1981).

Firstly, the follicular dendritic cells (FDC) of 1 ymph nodes and spleen germinal centres (Kotani et al., 1977), which possess, like tissue macrophages, $\mathrm{Fc}$ and $\mathrm{CP}_{3}$ receptors but lack cell surface Class II MHC molecules (Humphrey and Grennan, 1982). In the periphery they are highly phagocytic and migrate via afferent lymphatics into lymph node germinal centres carrying their phagocytosed antigenic load (Kotani et al., 1977, 1982).

The second lineage of accessory or antigen-presenting cells is the interdigitating reticulum cells (IDC) (Veerman, 1974) or dendritic cells (Steinman, 1981). Within 1ymphoid tissue they are localised to the lymph node paracortex and periarteriolar sheath of the spleen, but are absent from B-cell areas (Kamperdijk et al., 
1978). This cell population differs from FDC in that they all seem to possess cell surface Class II ( $\mathrm{Ia} / \mathrm{Dr}-1 \mathrm{kke}$ ) antigens which are thought to be essential for the successful presentation of antigen to T-cells (Kappler and Marrack, 1976). In contrast, Fc and $\mathrm{C}_{3}$ receptors are absent from the interdigitating reticulum or dendritic cells of man (Van Voorhis et al., 1982).

Other cell types, possibly variants of the macrophage lineage, may be involved in antigen presentation to B-cells. These cell types probably do not express Class II molecules and therefore presumably do not present antigen to T-cells (Roitt et al., 1985).

\section{I.E IMMUNOHISTOLOGICAL IDENTIFICATION OF LEUCOCYTE SUBPOPULATIONS}

\section{I.E.1 Introduction}

For over 100 years or so, pathologists have extracted an extraordinary amount of information from formaldehyde-fixed, paraffin embedded sections of human tissues by staining them with dyes such as haematoxylin and eosin and matching the patterns therein created with pre-existing diagnostic schemata. While these methods continue to be the primary form of analysis of tissue by the pathologist, it is clear that new methodologies can extract other types of information from the same material. Furthermore, there are occasions when additional information is required.

Histological studies using a variety of immunological reagents were, for many years, limited to the interested research 
worker. However, with improved equipment more refined techniques and high quality reagents, such studies are rapidly being integrated into general histopathological practice. It is now possible to stain for a wide variety of antigens, hormones, tumour markers, and immunoglobulins which not only enable a diagnosis to be made, but also give valuable additional information about the pathogenesis of a particular disease.

Certainly one of the most dramatic advances in recent years was the success of Kohler and Milstein (1976) in the in vitro production of antibodies with predetermined specificities, i.e. monoclonal antibodies. These antibodies, together with the more improved immunocytochemical techniques, have been successfully introduced into histopathological practice, especially in various aspects of cancer diagnosis, and research. The special and unique function of immunocytochemistry is to demonstrate the microscopic distribution of antigen in cells and tissues. These techniques utilising monoclonal antibodies are now currently used to study lymphocyte subpopulations in lymphoid tissue and other tissue sections in health and disease (Mason et al., 1982).

\section{I.E.2 Immunocytochemical Methods}

Immunocytochemical methods exploit the specific binding of antibody with its corresponding antigen in order to detect and localise cell or tissue antigens and render their presence directly visible utilizing a variety of labelled antibody methods. Histochemical methods were introduced in the later part of the 19th 
century by pathologists searching for more objective methods, aside from the examination of morphological features, for cellular recognition and diagnosis. Initially fluorescein was the on1y label available (Coons et al., 1941). The introduction of enzyme labels, such as horseradish peroxidase, was a major step forward (Nakane and Pierce, 1966) as they lack the major disadvantages of immunofluorescence, namely instability of stain, requirement for fresh tissue or live cells and specialised microscopy. In addition the background fluorescence is relatively difficult to exclude completely. More recently, immunological methods have been developed to bind the label to the antibody, e.g. the unlabelled antibody enzyme bridge method (Mason et al., 1969) and the peroxidase-antiperoxidase (PAP) complex method (Sternberger et al., 1970). In addition, numerous modifications of the original methods have been developed. The most important modifications are listed in Table 1.1 .

of these, immunoperoxidase methods are now the most extensively used methods in surgical pathology. The most common1y used peroxidase methods employ direct or indirect antibody conjugates or the peroxidase-antiperoxidase complex technique. In the direct peroxidase methods, horseradish peroxidase is directly conjugated to the primary antibody specific for the antigen under study. The labelled primary antibody is applied to the section and identifies the antigenic sites. The indirect methods involve the use of peroxidase conjugated antiserum with specificity directed to the globulin fraction of the primary antiserum (Nakane and Kawaoi, 1974). In this method the primary, unlabelled antiserum having specificity against the antigen is applied initially, followed by a 
TABLE I.1:

IMMUNOCYTOCHEMICAL METHODS

\begin{tabular}{ll}
\hline Method & Source \\
\hline
\end{tabular}

\section{Pure immunological methods}

1. Direct labelled antibody

2. Indirect labelled antibody

3. Unlabelled antibody enzyme bridge

4. Peroxidase-antiperoxidase (PAP) complex

5. Hapten labelled antibody

6. Simultaneous double-labelling

7. Labelled antigen

8. Immunogold staining
Nakane and Pierce (1966)

Nakane and Pierce (1966)

Mason et a1. (1969)

Stenberger et a1. (1970)

Jasani et al. (1981)

Mason and Sammons (1978)

Mason and Sammons (1979)

De Waele et al (1983)

\section{Mixed immunological/non immunological methods}

1. Labelled protein A

2. Avidin-biotin
Dubois Dalq et a1. (1977) Roth et al. (1978)

Guesdon et al. (1979)

Hsu and Raine (1981) 
second, labelled (peroxidase-conjugated), antibody from a second species, directed against the immunoglobulin components of the primary antiserum, the first antibody now acting as an immunoglobulin antigen. The primary antigenic site is thus revealed.

This technique is analogous to the indirect immunofluorescent method (Sternberger, 1970). It has certain advantages in that it is more sensitive than the direct method; it is more versatile by substitution of primary antisera against a wide variety of antigens; and it can be subjected to additional specificity controls by replacement of the primary antiserum by other antisera of the same origin but irrelevant specificity.

In the peroxidase-antiperoxidase technique, a soluble peroxidase-antiperoxidase complex rather than a peroxidase conjugated antiserum is used. The reaction sequence involves the application of the primary antiserum, an antiserum with specificity directed to the globulin fraction of the primary antiserum, and finally the peroxidase-antiperoxidase soluble complex which is prepared in the same species as the primary antiserum. This method was initially introduced by Sternberger et al. (1970) as an alternative to peroxidase conjugate and immunofluorescence procedures, with an additional advantage of enhanced sensitivity.

\section{I.E.3 Selection of the Immunohistological Staining Technique}

Several procedures, both immunological and non-immunological, are available to visualise antibodies that have 
bound to a tissue section. The indirect staining procedures are regarded as more sensitive than the direct procedures. As previously stressed, indirect methods have the advantage that the same procedure can be used in combination with different primary antisera, provided they are all raised in the same species. Methods using protein A or avidin-biotin complex as second steps appear to be promising but are still at developmental stage and more studies are required before a final judgement of their applicability can be reached (Bosman, 1983). Most workers accept that, among the indirect procedures, peroxidase-antiperoxidase is the most sensitive technique, followed by the indirect labelled antibody method (peroxidase conjugate method), and the fluorescent conjugate in that order. However, the validity of this assumption has been questioned (Boorsma et al., 1976) and many studies have shown that the peroxidase-antiperoxidase method is not significantly more sensitive than the indirect labelled antibody method (Heyderman and Neville, 1977; Bergroth et al., 1980). Furthermore, the indirect labelled antibody method is one stage shorter and the commercial peroxidase conjugates available today are considerably better in terms of strength of reactivity and lack of non specific background labelling.

Peroxidase markers are widely used due to the stability of 3,3-diaminobenzidine (DAB) product. Recently several means of enhancing the intensity of the DAB stain have been reported, such as by impregnation with silver salts (Gallyas et al., 1982) after the $\mathrm{DAB}$ reaction or by the addition of $0.01 \mathrm{M}$ imidazole to the $\mathrm{DAB}$ substrate solution (Straus, 1982). 
In view of the above, the choice therefore lies between the indirect conjugate method and the peroxidase antiperoxidase method. As in fact the end-results of the PAP method are not always better than those of the indirect conjugate method, and it is one stage longer, we have chosen the two-step indirect conjugate method. It is simple, practical, not time consuming, sensitive, versatile and subjected to additional specificity control by replacement of the primary antiserum by other antisera of the same origin but irrelevant specificity.

\section{I.E.4 Modes of Application of Immunoperoxidase Procedures}

Immunoperoxidase procedures have been used for the detection and demonstration of cell surface and intracellular antigens. The detection of antigen in different localisations is dependent on the mode of exposure of the cell population to the immunoperoxidase system. Tissue sections and fixed cell smears have been used for the demonstration of intracellular and cell surface antigen by immunoperoxidase methods. The use of cell surface antigens (surface markers) to identify and characterise subpopulations of cells has depended heavily on progress in developing monoclonal antibodies against cell surface determinants on different cells. An extensive range of monoclonal antibodies against human leucocyte surface markers has been developed. The combination of monoclonal antibodies to human leucocyte surface markers and immunoperoxidase techniques has made possible the study of lymphocyte subpopulations in frozen sections of normal 1ymphoid tissues (Poppema et al., 1981) 
providing important information regarding the anatomic compartmentalization of $\mathrm{B}$ - and T-1ymphocytes. These techniques also have been extended to the study of the distribution of 1 ymphocyte subpopulations in different systems in the body (Selby, 1981; Ritchie et al., 1984b; Allen and Hogg, 1985).

It should be stressed that there is, at present, a general consensus that frozen sections are to be preferred for the demonstration of lymphocyte differentiation antigens and surface immunoglobulin (Falini and Taylor, 1983), and that the antigenicity of $\mathrm{T}$ - and B-cell surface antigens defined by conventional or monoclonal antibodies can be destroyed in fixed paraffin embedded tissues, but not in frozen sections.

\section{I.F $\quad$ AIMS OF THE STUDY}

1) To investigate the presence and distribution of 1ymphocyte subsets and macrophages in the tissue and secretions of the normal male genital tract.

2) To ascertain if this pattern was altered in various clinical conditions, particularly in subfertile patients. 
CHAPTER II

MATERIALS AND METHODS 


\section{II.A COLLECTION AND PROCESSING OF TISSUE SPECIMENS}

\section{II.A.1 Normal Tissue Specimens}

Specimens of normal human testis, epididymis, vas deferens, prostate, seminal vesicles and urinary bladder were obtained over a two year period from eight fertile men with a mean age of 28.7 (19-40) years who had died in road traffic accidents. Relatives had given permission for multi-organ donation for transplantation and research purposes. Specimens of spleen and abdominal lymph nodes were also obtained from the same donors. In addition, specimens of the vas deferens were obtained from five fertile men requesting vasectomy, and specimens of the ureter were obtained from five donors for living related kidney transplant. Their mean age was 35.0 (31-39) years and $35.2(30-40)$ years respectively (Table II.1).

\section{II.A.2 Clinical Specimens}

These included surgical biopsies from the testis, the epididymis, the prostate, and the urinary bladder. It should be stressed that these biopsies were taken when there was sufficient remaining tissue after all the normal investigations necessary for the treatment of the patients had been performed. 
TABLE II.1:

SOURCE OF MALE GENITAL TRACT TISSUES USED IN THESE STUDIES

\begin{tabular}{|c|c|c|c|}
\hline $\begin{array}{l}\text { Source and/or } \\
\text { clinical problem }\end{array}$ & Tissues & $\begin{array}{l}\text { No. of } \\
\text { specimens } \\
\text { examined }\end{array}$ & $\begin{array}{l}\text { Average age } \\
\quad \text { (yrs) }\end{array}$ \\
\hline \multirow[t]{5}{*}{$\begin{array}{l}\text { Multi-organ transplant } \\
\text { donors }\end{array}$} & Testis & 8 & \\
\hline & Epididymis & 8 & \\
\hline & Vas deferens & 8 & \\
\hline & Prostate & 8 & $28.7(19-40)$ \\
\hline & $\begin{array}{l}\text { Seminal vesicles } \\
\text { and ampulla of } \\
\text { the vas }\end{array}$ & 4 & \\
\hline Oligozoospermia & Testis & 10 & $27.5(21-35)$ \\
\hline \multirow[t]{2}{*}{$\begin{array}{l}\text { Bilateral epididymal } \\
\text { obstruction }\end{array}$} & Testis & 6 & $35.6(23-45)$ \\
\hline & Epididymis & 3 & $37.0(32-44)$ \\
\hline \multirow[t]{2}{*}{$\begin{array}{l}\text { Unilateral epididymal } \\
\text { obstruction }\end{array}$} & Testis & 2 & 31 \\
\hline & Epididymis & 1 & 31 \\
\hline \multirow[t]{2}{*}{ Vasectomy reversal } & Testis & 6 & $34.6(31-39)$ \\
\hline & Epididymis & 2 & $33.0(32-34)$ \\
\hline $\begin{array}{l}\text { Benign prostatic } \\
\text { hypertrophy (BPH) }\end{array}$ & Prostate & 8 & $70.6(54-78)$ \\
\hline
\end{tabular}




\section{II.A.2.1 Testicular biopsies from subfertile patients}

These were obtained from 24 patients with a mean age of $33.7 \pm 6$ years undergoing investigation and/or treatment of male infertility (Table II.1). Ten biopsies were taken from men who, after three semen analyses, were found to have a sperm density of less than $10 \mathrm{million} / \mathrm{ml}$. Biopsies were also taken from eight other patients, six of whom (H.W., J.K., A.S., B.G., S.B., and B.W.) were diagnosed as having obstructive azoospermia and two (A.H. and R.T.) as having unilateral testicular obstruction. The diagnosis of unilateral testicular obstruction was established by exploratory scrototomy (Figure II.1). A further six biopsies were taken from six previously healthy men (F.T., J.B., I.G., I.P., D.D., and S.L.) who had undergone vasectomy. The mean duration of obstruction in these latter patients was 3.6 (3-6) years. Biopsies from patients with testicular obstruction were taken at the time of epididymo-vasostomy or at the time of vasectomy reversal. In all these patients agglutinating sperm antibodies were measured in serum and in seminal plasma before biopsy using the tray slide agglutination test (TAT) (Friberg, 1974).

\section{II.A.2.2 Epididymal biopsies}

These were obtained from three of the patients with obstructive azoospermia (H.W., J.K. and B.G.) and from one of the patients with unilateral obstruction (R.T.) and from two of the patients with vasectomy reversal (I.G. and D.D.). The mean age of these six patients was 34.6 (31-44) years (Table II.1). 


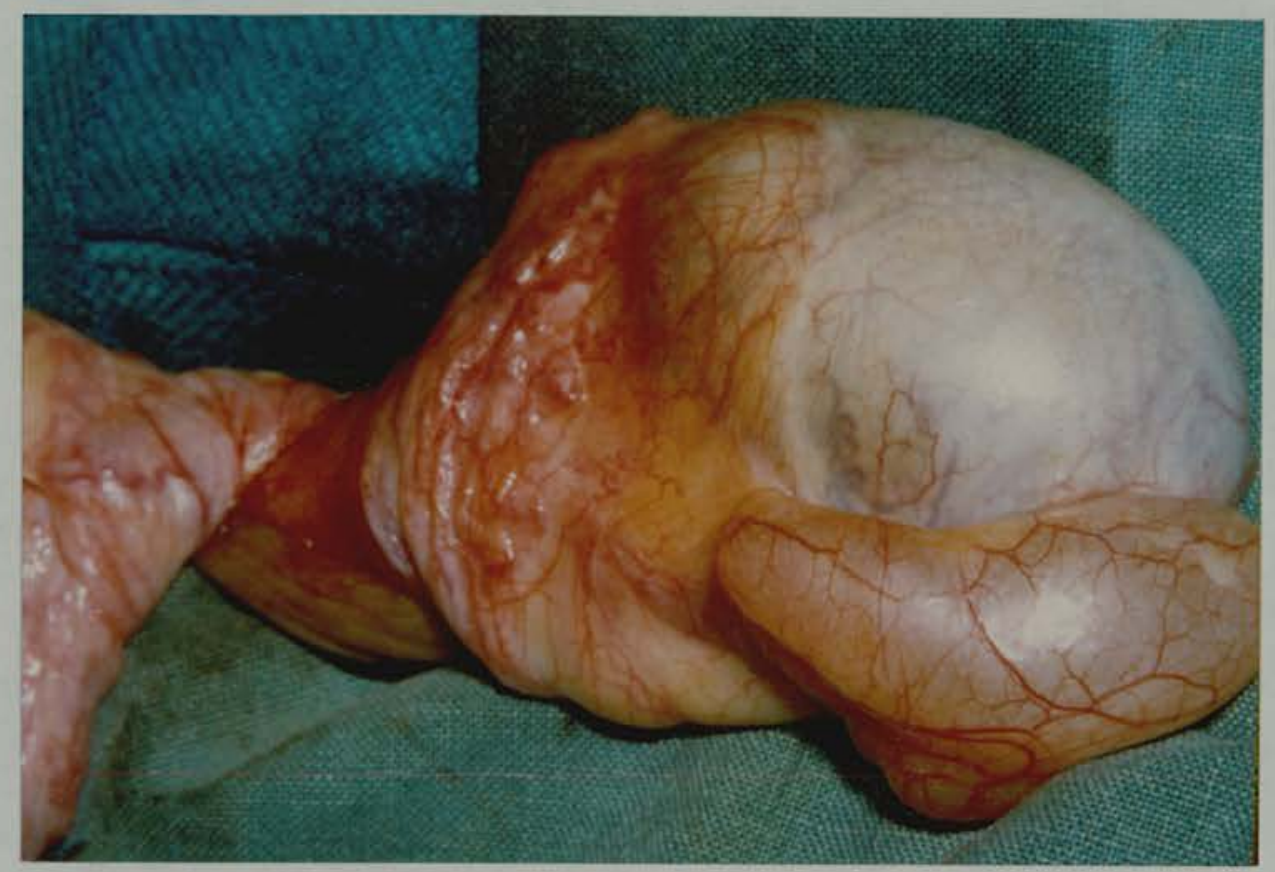

Figure II.1: Exploratory scrototomy revealing unilateral epididymal obstruction 


\section{II.A.2.3 Prostatic biopsy specimens}

Prostatic biopsy specimens were obtained following open operations for benign prostatic hypertrophy (BPH). These were obtained from seven patients with a mean age of 70.6 (64-78) years. A further biopsy was taken from a patient (54 years) who was diagnosed as having chronic prostatitis, in addition to $\mathrm{BPH}$.

\section{II.A.2.4 Urinary bladder biopsy specimens}

Endoscopic bladder biopsies were taken from three patients with histologically proved carcinoma in situ of the urothelium. The age range was 48 to 72 years, with a mean of 66 years.

\section{II.A.3 Tissue Preparation Techniques}

\section{II.A.3.1 Handling of tissue specimens}

Immediately after surgical removal, every normal tissue specimen was sliced into small representative blocks $5-10 \mathrm{~mm}$ in thickness. Every tissue sample was put in a plastic universal tube which was marked with the name of the patient, the hospital number and the date of the operation.

The fresh unfixed tissue samples were snap frozen by immersing the tubes containing the tissue samples directly in liquid nitrogen within 1-2 hours of surgical removal. The tissues were then stored at $-70^{\circ} \mathrm{C}$ until used. 


\section{II.A.3.2 Cryostat sectioning}

Five um cryostat sections were cut from each tissue sample/block, using a Teddington type QR cryostat (Brights Refrigeration Service, Huntington, England) (Figure II.2). Serial sections were picked up on alcohol washed glass slides and then air dried for 1-2 hours under a hair dryer.

\section{II.A.3.3 Fixation and storage of frozen sections}

After air drying, sections were fixed in acetone for 10 minutes at room temperature and air dried again. The sections were either used immediately or wrapped in aluminium foil and stored at $-20^{\circ} \mathrm{C}$ until used.

\section{II.B COLLECTION OF SEMEN SPECIMENS}

\section{II.B.1 Patients}

This study was carried out on 81 men, 69 of whom were patients with a mean age of $30.1 \pm 4.8$ years attending the infertility clinic complaining of an infertile marriage. The remainder were fertile men with a mean age of $30.8 \pm 10.2$ years attending for vasectomy. The fertile men had fathered at least two children and at the time of testing had one child under two years of age. All were given clearly written instructions about the 


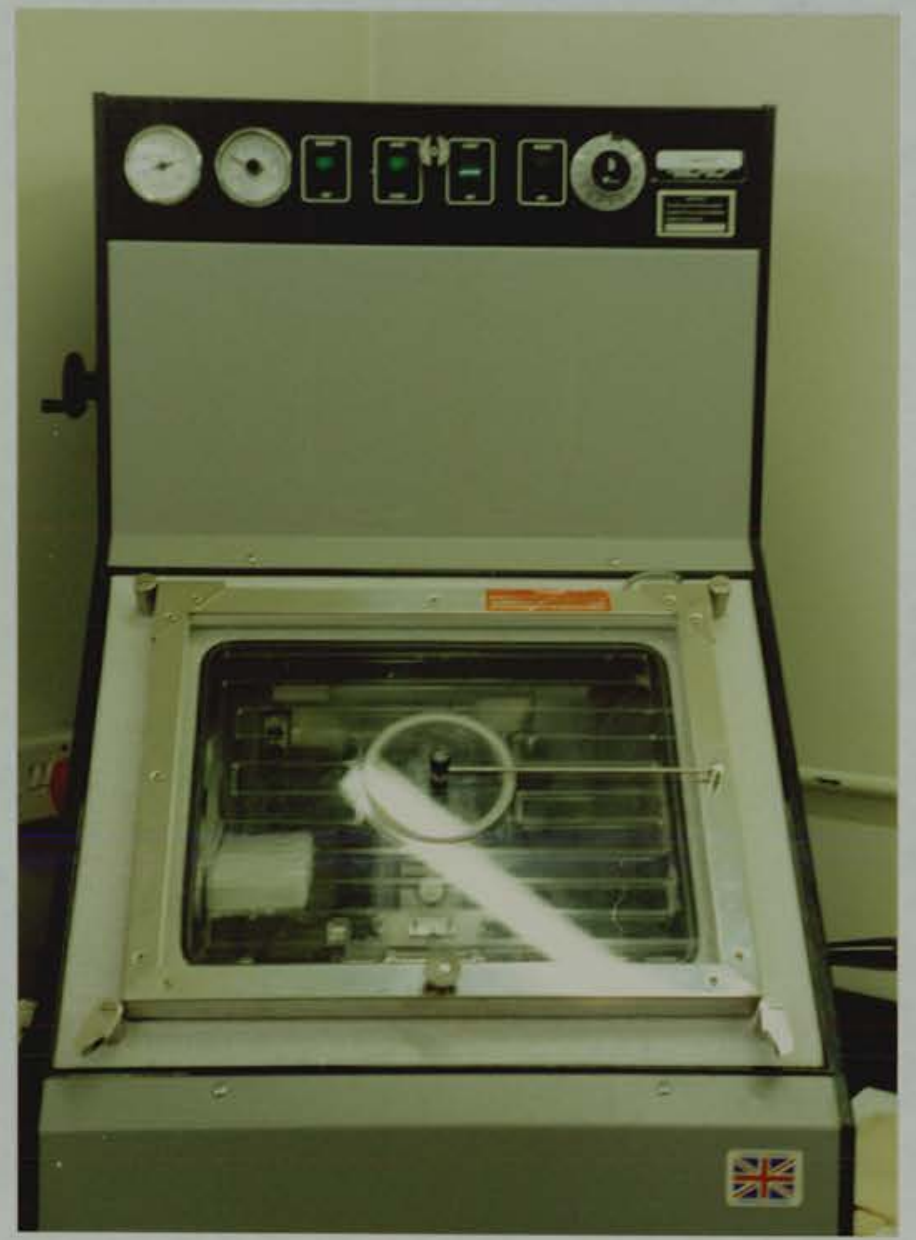

Figure II.2: A Teddington type QR cryostat 
production and delivery of the semen specimens. In addition a full medical history was obtained and a complete physical examination was performed.

\section{II.B.2 $\underline{\text { Semen }}$}

Semen was collected by masturbation into sterile plastic containers after three days of sexual abstinence. Using sterile precautions two aliquots were extracted from each specimen, one for standard semen analysis and the other for bacterial cultures. The remainder of the specimen was prepared for immunocytochemical staining.

Semen analysis was performed as described in the World Health Organization (WHO) Laboratory Manual (Belsey et al., 1980). This included sperm density, motility and morphology. Anti-sperm antibody measurement was done using the TAT (Friberg, 1974) and the MAR (mixed antiglobulin reaction) tests (Jager et al., 1978).

\section{II.B.3 Microbiological Screening of Semen Specimens}

This was performed in the University Department of Microbiology by $\mathrm{Dr} \mathrm{H}$. Young. All the specimens were tested for aerobic and anaerobic micro-organismms, Ureaplasma urealyticum and Chlamydia trachomatis. The methods used have been described by Young et al.( 1983) and were briefly as follows. 
Bacterial cultures were performed by serially diluting 100 ul semen in ten-fold steps in peptone water up to a dilution of $10^{5}$ and spreading 20 ul of each dilution onto blood agar and gentamycin blood agar. The blood agar plates were incubated in $5 \% \mathrm{CO}_{2}$ for 24 hours. The gentamycin blood agar was incubated anaerobically for 48 hours. The identification of the aerobic and anaerobic organisms was based on the criteria outlined by Cowan (1974) and on the methods of Duerden et al. (1980) respectively. Chlamydia trachomatis was isolated by the method of Thomas et al. (1977) with cycloheximide-treated cells. For the isolation and identification of Ureaplasma urealyticum, $200 \mathrm{ul}$ semen were used to initiate a series of ten-fold dilutions in $1.8 \mathrm{ml}$ volumes of TALC colour change medium (Young et al., 1981). The final dilution was $10^{5}$. The presence of Ureaplasma urealyticum was indicated by a change of colour of the medium. One colour changing unit (CCU) of activity was defined as the highest dilution of Ureaplasma suspension that produced a colour change (Taylor-Robinson et al., 1971). After allowing for the appropriate dilutions, the results were expressed as $\mathrm{CCU} / \mathrm{ml}$ of semen.

\section{II.B.4 Processing of Semen Specimens}

\section{II.B.4.1 Introduction}

Immunoperoxidase methods of staining have been extensively used in the study of tissue sections and subsequently this approach has been extended to the study of fixed smears of cell suspensions 
in clinical haematology and/or diagnostic cytology (Nadji, 1980; Moir et a1., 1983; Erber et al., 1984). In this context immunoperoxidase staining was felt to offer a similar advantage, that is, immunocytochemical detection of leucocyte subpopulations in fixed smears of cell suspensions of the ejaculate. Therefore, to establish this technique single cell suspensions were prepared of blood leucocytes, ejaculate and mixtures of both. Cytocentrifuge smears were then prepared from each suspension and stained, immunocytochemically, to identify leucocyte subpopulations. Having established the optimum conditions of the technique, in particular the preparation of single cell suspensions of the ejaculate with minimal loss of, and damage to, the cells, semen specimens from subfertile patients were stained similarly. Leucocyte smears were used throughout the study, in addition to establishing the technique, as positive controls.

\section{II.B.4.2 Preparation of a single cell suspension of the ejaculate}

The steps involved in the preparation of a single cell suspension from the ejaculate were as follows:

1) One $\mathrm{ml}$ of the freshly collected ejaculate was mixed with $9 \mathrm{ml}$ of Hanks Balanced Salt Solution (HBSS) (Gibco), calcium and magnesium free, in a $10 \mathrm{~m} 1$ conical centrifuge tube (Sterilin tube, $10 \mathrm{ml}$. Feltham, Middlesex, England), and centrifuged at $200 \times \mathrm{g}$ for 10 minutes at room temperature.

2) The supernatant was decanted and the cell pellet resuspended in $10 \mathrm{ml} \mathrm{HBSS}$ and the total sperm count was determined using a Neubauer counting chamber. 

10 minutes and finally the cell pellet was resuspended at a concentration of 5-10 million sperm/m1 in HBBS or in RPM1-1640 medium (Gibco) supplemented with $10 \%$ fetal calf serum (RPM1/FCS).

It should be noted that this procedure, which removed seminal plasma proteins and resulted in minimal loss of, and damage to cells, was adopted following extensive background studies.

Counting cells in suspension was performed using a haemocytometer (Neubauer) counting chamber. Immediately before the second wash, $100 \mathrm{ul}$ of the cell suspension were mixed with $100 \mathrm{ul}$ of $10 \%$ Nigrosine dye in a small clean glass tube. After standing for 3-5 minutes, a drop of the diluted well-mixed cell suspension was transferred to the counting chamber, covered with a coverslip and allowed to settle. Counts of live cells, i.e. non stained, were determined in duplicate and the mean was taken. The total number of viable cells in a $4 \mathrm{~mm}^{2}$ area (the four large corner squares) were counted and the number obtained multiplied by 2 (the dilution) $\mathrm{x} 10$ (the depth) and divided by $4\left(\mathrm{~mm}^{2}\right) \times 10^{3}$ to give the number of viable cells per $10^{6}$ per $\mathrm{ml}$ of suspension.

\section{II.B.4.3 Preparation of cytocentrifuge smears of washed ejaculate}

One hundred ul of the cell suspension, prepared as previously described, were placed in each cytospin block and spun down onto alcohol cleaned glass slides using a Shandon cytocentrifuge (Shandon-Elliot, Southern Instruments Ltd., England) 
(Figure II.3). This was achieved by spinning at $800 \mathrm{rpm}$ for 10 minutes at room temperature.

The smears were circled with a diamond marker, air dried for one hour under a hair dryer and then fixed in acetone for 10 minutes at room temperature. The air-dried, acetone-fixed smears were either stained immediately or stored, wrapped in aluminium foil and kept at $-20^{\circ} \mathrm{C}$ until used.

\section{II.B.4.4 Preparation of mononuclear and polymorph nuclear leucocyte smears from human peripheral blood}

Mono-Poly-Resolving Medium (M-PRM), obtained from Flow Laboratories Ltd., Irvine, Scotland, was used for the separation of white blood cells. This medium is composed of polysaccharide (Ficol1 400) and a radio opaque contrast medium (Hypaque 85) in a specific ratio to yield a density of $1.114 \pm 0.002$. It enables the resolution of both mononuclear and polymorph nuclear leucocytes into two distinct bands in one step. Both bands are also free of contaminating erythrocytes (Ferrante and Thong, 1980).

The procedure involved in the isolation of mononuclear and polymorph nuclear leucocytes from peripheral blood was essentially similar to that described by the manufacturer and involved the following steps:

1) Human venous blood, $15-20 \mathrm{ml}$, was obtained from a healthy donor by venipuncture and mixed with $2 \mathrm{ml}$ of $2 \%$ EDTA in a $25 \mathrm{ml}$ universal tube.

2) Five $m 1$ of the fresh, anticoagulant-treated blood were layered over $4 \mathrm{ml}$ of $\mathrm{M}-\mathrm{PRM}$ in a $10 \mathrm{ml}$ centrifuge tube, and 


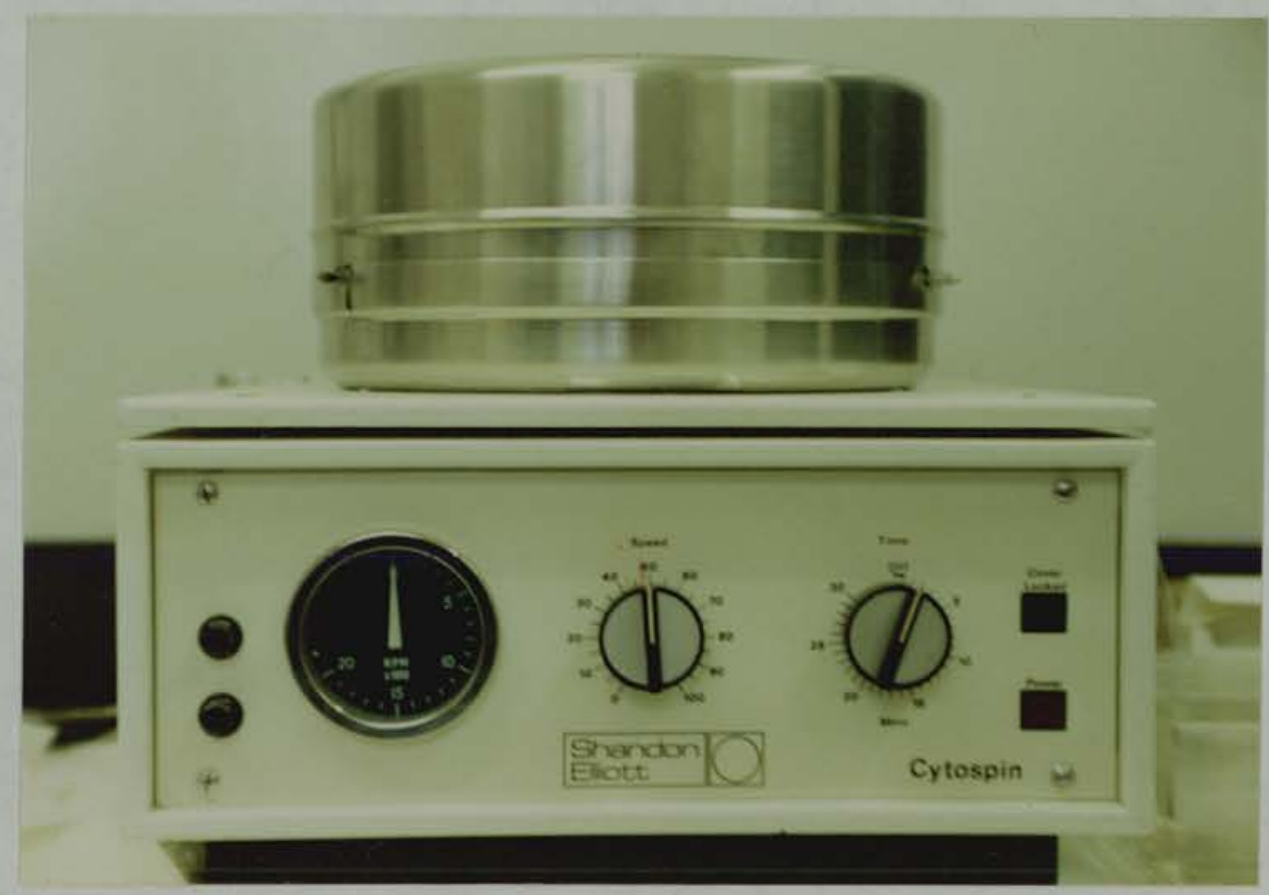

Figure II.3: A Shandon cytocentrifuge 
centrifuged at $300 \times \mathrm{g}$ for 30 minutes at room temperature.

3) The mononuclear leucocyte band (at the plasma-medium interface) and the polymorph nuclearfleucocyte band (below the interface) were aspirated with separate pipettes and transferred into separate clean tubes.

4) The separated cell preparations were washed twice in $10 \mathrm{~m} 1$ of HBSS and harvested by centrifugation at $200 \mathrm{x} g$ for 10 minutes.

5) Every cell pellet was resuspended at a concentration of 2-5 million/ml in HBSS or in $\mathrm{RPMl} / \mathrm{FCS}$.

6) Each cell suspension and a mixture of both were used to prepare cytocentrifuge smears as described previously.

Mononuclear cells were also isolated using the method of Boyum (1968) with minor modifications. The fresh blood, with added anticoagulant, was diluted 1:1 with HBSS (calcium and magnesium free). The diluted blood was layered over $15 \mathrm{ml}$ of "Lymphocyte separation medium", obtained from Flow Laboratories, Irvine, Scotland, in $25 \mathrm{ml}$ centrifuge tubes. The tubes were centrifuged at $400 \mathrm{x} \mathrm{g}$ for 25 minutes and the cells at the interface region aspirated and then washed twice in HBSS. Finally, the cells were resuspended at a concentration of $3-5 \mathrm{million} / \mathrm{ml}$ in HBSS or in RPM1/FCS medium.

In order to identify phagocytic cells, smears were also prepared from peripheral blood leucocyte suspensions preincubated with fluorescent latex microspheres. An aliquot of the leucocyte rich cell suspension ( 5 million cells $/ \mathrm{ml}$ ) was incubated with an excess of fluorescent microspheres at $37^{\circ} \mathrm{C}$ for 1 hour in RPM1/FCS. The particles were obtained from Polysciences Inc., Warrington, P.A. 
as Fluoresbrite carboxylate microspheres $(2.5 \%$ solid latex with a diameter of $0.73 \mathrm{um})$. The cells were then washed three times using low-speed centrifugation at $200 \mathrm{x} g$ for 10 minutes, so that free uningested latex particles did not pellet and were discarded in the wash supernatants. Finally, the cells were resuspended in HBSS to prepare cytocentrifuge smears.

\section{II.C MONOCLONAL ANTIBODIES}

A range of mouse monoclonal antibodies, with well-defined specifities to human lymphoreticular cell surface markers, was used in this study. These antibodies are detailed in Table II.2, which also indicates their source, specificity and origins.

\section{II.C.1 Preparation and Storage of Monoclonal Antibodies}

The monoclonal antibodies purchased from Becton Dickinson were supplied as 100 ug of purified immunoglobulin in $0.5 \mathrm{ml}$ of phosphate buffered saline, PBS, (200 ug/ml) with the exception of the anti-Leu $3 \mathrm{a}$ and anti-Leu $2 \mathrm{a}$ monoclonal antibodies which were at

a concentration of $100 \mathrm{ug} / \mathrm{ml}$. Each antibody was diluted to a concentrate of $20 \mathrm{ug} / \mathrm{ml}$ or volume in medium RPM1-1640 containing $5 \%$ fetal calf serum and $0.1 \%$ sodium azide. The diluted antibody was divided into $0.5 \mathrm{ml}$ aliquots and kept in small plastic tubes (Nunc Tubes, InterMed, Denmark) at $-20^{\circ} \mathrm{C}$ until used. 
TABLE II.2:

MONOCLONAL ANTIBODIES TO HUMAN LEUCOCYTE SURFACE ANTIGENS USED IN THESE STUDIES

\begin{tabular}{|c|c|c|c|c|c|}
\hline Monoclonal antibody & $\begin{array}{c}\text { CD } \\
\text { Number }\end{array}$ & $\begin{array}{l}\text { Molecular } \\
\text { weight in } \\
\text { kilo dalton }\end{array}$ & Specificity & Source & Reference \\
\hline Anti-leucocyte (HLE-1) & - & $180-220$ & All leucocytes & BD & Beverley, 1980 \\
\hline T6 & CD 1 & 49 & $\begin{array}{l}\text { Thymocytes } \\
\text { Langerhans cells }\end{array}$ & $\mathrm{CE}$ & Reinherz et al., 1980d \\
\hline T11 & CD 2 & 50 & All T-cells & $C E$ & Kamoun et al., 1981 \\
\hline $\begin{array}{ll}\text { T3 } & \text { ) } \\
\text { Leu } 4 & \text { ) }\end{array}$ & $\mathrm{CD} 3$ & $19-29$ & $\begin{array}{l}\text { All T-cells } \\
\text { All T-cells }\end{array}$ & $\mathrm{BD}$ & $\begin{array}{l}\text { Reinherz et al., 1980d } \\
\text { Ledbetter et al., } 1981\end{array}$ \\
\hline $\begin{array}{l}\text { T4 } \\
\text { Leu } 3 a \\
\text { Leu } 3 a+b)\end{array}$ & CD 4 & $56-62$ & Helper/inducer T-cells & $\begin{array}{l}\mathrm{CE} \\
\mathrm{BD} \\
\mathrm{BD}\end{array}$ & $\begin{array}{l}\text { Reinherz et al., } 1979 \\
\text { Evans et al., } 1981 \\
\text { Evans et al., } 1981\end{array}$ \\
\hline $\begin{array}{ll}\mathrm{T} 8 & \text { ) } \\
& \text { ) }\end{array}$ & CD 8 & $32-34$ & $\begin{array}{l}\text { Suppressor/cytotoxic } \\
\text { T-cells }\end{array}$ & $\mathrm{CE}$ & Reinherz et al., $1980 \mathrm{c}$ \\
\hline Leu $2 a \quad ）$ & & & & $\mathrm{BD}$ & Engleman et al., 1981 \\
\hline $\begin{array}{ll}\text { Leu } 11 \mathrm{~b} & \text { ) } \\
\text { Leu } 7 & \text { ) }\end{array}$ & $\mathrm{CD} 16$ & $\begin{array}{l}50-70 \\
110\end{array}$ & $\begin{array}{l}\text { Large granular } \\
\text { lymphocytes (NK-cells) }\end{array}$ & $\begin{array}{l}\mathrm{BD} \\
\mathrm{BD}\end{array}$ & $\begin{array}{l}\text { Lanier et al., } 1983 \\
\text { Abo and Balch, } 1981\end{array}$ \\
\hline $\begin{array}{ll}\text { B1 } & \text { ) } \\
\text { Leu } 14 & \text { ) }\end{array}$ & CD 20 & 35 & B-cells & $\begin{array}{l}\mathrm{CE} \\
\mathrm{BD}\end{array}$ & Stashenko et al., 1980 \\
\hline Interleukin-2 receptors & $\mathrm{CD}-25$ & 55 & Activated T-cells & $\mathrm{BD}$ & Uchiyama et al., 1981 \\
\hline Leu M3 & - & - & ) & BD & $\begin{array}{l}\text { Dimitriu-Bona et al., } \\
1983\end{array}$ \\
\hline $\begin{array}{l}3.9 \\
24\end{array}$ & $\begin{array}{c}\mathrm{CD} 11 \mathrm{C} \\
-\end{array}$ & $\begin{array}{l}150,95 \\
175\end{array}$ & Monocytes/Macrophages ) & $\begin{array}{l}\text { ICR } \\
\text { ICR }\end{array}$ & $\begin{array}{l}\text { Hogg et al., } 1986 \\
\text { Hogg and Selvendran, } \\
1985\end{array}$ \\
\hline HLA-DR & - & $28-34$ & $\begin{array}{l}\text { Non-polymorphic } \\
\text { Class II antigens }\end{array}$ & $\mathrm{BD}$ & Lampson and Levy, 1980 \\
\hline
\end{tabular}

$\mathrm{BD}=$ Becton Dickinson Lab. Impex Ltd., Twickenham, England.

$\mathrm{CD}=$ Coulter Electronics Ltd., Luton, England.

ICR = Imperial Cancer Research Fund (Macrophage Laboratory), London, England.

$C D$ = Cluster of differentiation as proposed by the Committee on Human Leucocyte Differentiation Antigens (1984). 
Coulter clone monoclonal antibodies were supplied as lyophilized reagents in vials. The contents of each vial was sufficient for 100 tests. Each lyophilized antibody was reconstituted by adding $0.5 \mathrm{ml}$ of distilled water. The reconstituted antibody was then diluted in $10 \mathrm{~m} 1 \mathrm{PBS}$ (see Appendix A) with $2 \%$ fetal calf serum to yield one half of the working dilution as recommended by the manufacturers. The diluted antibody was aliquoted $(0.5 \mathrm{ml})$ into small Nunc plastic tubes and kept at $-20^{\circ} \mathrm{C}$ until used.

Prior to use, the frozen antibody was allowed to thaw at room temperature after which it was diluted to its final working dilution, for staining. The diluent used was $2 \%$ normal rabbit serum (NRS) in $0.05 \mathrm{M}, \mathrm{pH} 7.6$, Tris-buffered saline (TBS) (see Appendix A).

\section{II.C.2 Titration of Antibody Concentration}

Optimal dilutions of the reagents and the reliability of the staining procedure were assessed by repeated attempts at staining frozen sections of normal human spleen, lymph nodes and tonsils, as well as cytocentrifuge smears of human peripheral blood leucocytes. Serial sections of lymphoid tissues and leucocyte smears were stained with increasing dilutions of each monoclonal antibody using a range of concentration from $1: 40$ to $1: 320$, followed by a fixed concentration of the secondary antibody. Titration of the second step reagent, the peroxidase conjugated antibody, was performed using a range of concentration from $1: 25$ to $1: 200$ on sections and 
smears preincubated with fixed concentrations of the primary antibodies. The optimal combination of dilutions, represented by the slides with the most intense specific staining and the least amount of background staining, was selected.

\section{II.C.3 Other Cytochemical Reagents}

\section{II.C.3.1 Peroxidase conjugate}

Immunoperoxidase staining involves the use of antibodies and the enzyme peroxidase. Peroxidase reacts with its specific substrate, hydrogen peroxide $\left(\mathrm{H}_{2} \mathrm{O}_{2}\right)$, to form a primary complex. This complex reacts with an electron donor to produce the end products of the reaction, a coloured molecule and and water. In the indirect application an unconjugated antibody will bind to the antigen in the specimen. To localise this attachment, a peroxidase conjugated antibody is needed to bind the first antibody. High quality peroxidase-conjugated anti-mouse immunoglobulin reagents are now available commercially. The peroxidase conjugated rabbit anti-mouse immunoglobulin obtained from Dakopatt (Copenhagen, Denmark) was used in this study. Normal human serum was added at a concentration of $1: 25$ to this reagent in order to block crossreactivity against human immunoglobulin. 


\section{II.C.3.2 3-3'diaminobenzidine tetrahydrochloride (DAB)}

DAB was introduced by Graham and Carnovsky (1966) for histochemical detection of peroxidase. DAB is an electron donor to the peroxidase/hydrogen peroxide complex. The free bonds of the oxidation product of $\mathrm{DAB}$ react with each other to form an insoluble brown phenazine polymer. DAB is very toxic and possibly carcinogenic.

DAB was obtained, in sealed vials each containing $10 \mathrm{mg}$, from BDH Chemicals, Poole, Dorset. Using a disposable syringe, the contents of a vial were dissolved in $20 \mathrm{ml}$ of TBS to give a final concentration of $0.5 \mathrm{mg} / \mathrm{ml}(0.05 \% \mathrm{w} / \mathrm{v})$. A fresh $\mathrm{DAB}$ solution was used before each session of staining. Immediately before use $20 \mathrm{ul}$ of $30 \% \mathrm{H}_{2} \mathrm{O}_{2}$ (100 volume) was added to give a final concentration of $0.01 \%$. Because of the inherent toxicity of the DAB, a mask and gloves were always worn while it was being dispensed/handled.

\section{II.D IMMUNOPEROXIDASE STAINING OF TISSUE SECTIONS AND}

\section{CELL SMEARS}

\section{II.D.1 Procedure}

The indirect labelled (two stage) antibody method of Nakane and Pierce (1966) was applied with modifications. The steps involved were as follows: 
Preparation of slides (sections/smears) for staining:

Immediately before staining the stored slides were removed from the freezer, brought to room temperature and unwrapped. The slides were air dried for 10 minutes under a hair dryer, and then washed in $0.05 \mathrm{M}$, $\mathrm{pH} 7.6$, TBS for 5 minutes.

\section{Incubation with monoclonal antibodies:}

The serial sections and/or the smears were overlayed with NRS diluted 1 in 4 in TBS for 10 minutes, to block non specific binding of protein, after which they were drained off. The sections/smears were then overlayed with the appropriate working dilutions of the primary mouse monoclonal antibodies and incubated for 30 minutes in moist chambers.

\section{Controls:}

Negative control sections were either treated with TBS only or with another irrelevant primary antiserum to a different antigen, e.g. an antiprostatic acid phosphatase antibody. As positive controls, the primary antibodies were applied to frozen sections of human spleen, lymph nodes and tonsils or to leucocyte smears (see specificity controls II.D.2).

\section{Incubation with secondary antibody:}

After washing for 5 minutes in two changes of TBS, to remove unbound antibody, the sections/smears were overlayed with the peroxidase conjugate rabbit anti-mouse antibody containing normal human serum, diluted 1 in 50 in TBS. Incubation was continued for 30 minutes, followed by washing in two changes of TBS. 


\section{Development of the peroxidase reaction:}

The final (colour) reaction was achieved by incubating the sections/smears for 5-8 minutes in $0.01 \% \mathrm{H}_{2} \mathrm{O}_{2}$ and $0.05 \% \mathrm{DAB}$, freshly prepared in TBS. The staining reaction and its progress was followed under a microscope to minimise background staining. The reaction was terminated by rinsing the slides in tap water. To enhance and increase the intensity of staining, the slides were incubated in $2 \% \mathrm{CuSO}_{4}$ in distilled water for 4 minutes, and washed in tap water.

\section{Counter-staining:}

The sections/smears were counter-stained in Mayer's haematoxylin for 30-60 seconds, washed in tap water and mounted in Apathy's aqueous mounting medium. Alternatively, sections were dehydrated in graded alcohol, cleared in xylene and mounted in DPX (Distrene Plastisier (dibutyl phthalate) Xylene) (Raymond A. Lamb, Waxes and General Laboratory Supplies, London).

It should be noted that the above procedure was adopted following extensive background studies performed to achieve maximal intensity of specific staining with absent or minimal background staining. No attempt was made to routinely block the endogenous peroxidase activity in this study since this was not anticipated to be a problem within the tissues to be studied. However, occasionally we preincubated some sections and smears with $0.3 \% \mathrm{H}_{2} \mathrm{O}_{2}$ in absolute methanol for 10 minutes to block endogenous peroxidase activity . 


\section{II.D.2 Specificity}

Strict specificity of an immunochemical reaction is one of the most important prerequisites for reliable immunocytochemistry. Originally, all the monoclonal antibodies used in this study were obtained as purified immunoglobulins and with well defined specificities (for references see Table II.2). Furthermore, a monoclonal antibody reacts with only one single antigenic determinant and this characteristic should theoretically guarantee absolute specificity. However, specificity was conventionally tested by including a standard set of control sections with each staining sequence.

Specificity controls:

The following control sections were used:

1. Sections in which the primary antibody was omitted and treated only with TBS (buffer), while other steps are kept the same. This is to ensure that the second step reagent, the peroxidase conjugated rabbit anti-mouse antibody shows no affinity for the tissue section.

2. Sections incubated with a "non-immune serum", normal mouse serum, or with an unrelated antibody, a mouse antiprostatic acid phosphatase antibody (which has no specificity for any antigens in the tissue under investigation). This is to assess whether binding of immunoglobulin in the primary antibody is causing non specific staining or to ascertain whether the immuno-cytochemical reagents alone cause staining of the tissue, i.e. Method Specificity (Pool et a1., 1983). 
3. Staining tissue sections taking a particular antigen to which the primary antibody is specific, e.g. normal testicular tissue sections stained with antilymphocyte antisera. This is to demonstrate that positive staining is due to specific antibody and is not due to contaminated antibody or due to non specific binding, i.e. serum specificity.

This was also tested by the use of at least one alternative antibody of the same specificity, i.e. staining suppressor T-cells by monoclonal antibodies anti-Leu $2 \mathrm{a}$ and T8. If two antisera from independent sources give the same staining patterns, this provides compelling evidence that the reaction is specific, since the two reagents are unlikely to contain the same contaminating antibody.

4. Sections of normal lymphoid tissue: spleen, lymph nodes and tonsil (against which the reactivity of each antiserum has already been evaluated) were always included as a positive control. This is to ensure that the staining technique is working and also that the method of tissue processing is satisfactory for that antigen.

5. Sections incubated only with diaminobenzidine/hydrogen peroxide substrate to check for endogenous peroxidase activity. 


\section{II.D.3 Interpretation of Labelling Results}

Immunoperoxidase staining in combination with a specific antibody enables localization of cellular antigens via the enzyme peroxidase. The substrate $(D A B)$ is added which will react with the enzyme and precipitate at the site of antigen representing specific positive staining. Positive staining for surface markers appears on the periphery of the cell. Intracytoplasmic antigens may be visualised in particular when cell smears are stained (Mason et al., 1986). Interpretation of the staining results should be made based on the presence of positive and negative controls, allowing comparison of the specific and non specific staining pattern of the unknown with that of the control. Interpretation should also be made based on properly fixed specimens where the antigen is preserved and also the morphology.

Artifacts/as precipitates, tissue artifact, cell artifact and specific background staining might interfere with correct interpretation. This could be prevented by proper handling of the specimens and proper staining techniques. 


\section{II.E IMMUNOHISTOLOGICAL ANALYSIS AND SCORING}

\section{II.E.1 Tissue Sections}

Sections were examined by light microscopy (Car1-Zeiss model) using a x 160 magnification. The positively labelled cells were counted within the epithelium, within the subepithelial connective tissue (the lamina propria) and within the interstitial connective tissue of the urogenital organs. In every case the number of cells reacting with any individual monoclonal lymphocyte antibody was determined in 10 different fields in identical areas of the serial sections. In the case of the epididymis, the positively stained lymphocytes were counted in a minimum of 20 cross-sections of each region of the epididymis or prostatic glands, using an eye-piece with a graticule. The total number of the mucosal epithelial cells were also determined by counting cell nuclei. At least 200 labelled cells (with any individual antibody) and 1000 mucosal epithelial cells were located in similar areas of the serial sections. On the basis of these counts the following data were obtained:

a) the percentage of mucosal epithelial cells which stained with the individual monoclonal antibodies and

b) the ratio of the suppressor/cytotoxic T-cells to the helper/ inducer T-cells in various histological compartments of each organ.

The results are expressed as the mean \pm standard deviation (S.D.) of the number of positively stained cells per 100 mucosal 
epithelial cells (for the epithelium), or per cross section of the epididymal duct or the vas deferens (for the lamina propria), or per single field ( $0.8 \mathrm{~mm}^{2}$ tissue) for the interstitial tissues.

\section{II.E.2 Ejaculate Smears}

Smears were examined using a magnification of $x 320$. The cells which stained with any individual monoclonal antibody were counted in 10 different fields for each smear. The average was taken and multiplied by the dilution (the number of $\mathrm{ml}$ in which the cell pellet was finally resuspended). Results were expressed as the mean count together with the standard deviation (S.D.). The resulting numbers of leucocytes reported in the tables and the figures do not have any independent meaning but they do allow comparison between patients and distinction of those with high or low leucocyte numbers.

\section{II.E.3 Statistical Analysis}

Selection of the appropriate statistical methods and statistical analysis was undertaken by Dr R.A. Elton of the Medical Statistics Unit, University of Edinburgh.

Wilcoxon rank sum tests were used to compare the mean leucocyte or 1ymphocyte subset counts in the normal donors and the different groups of patients. Comparison of lymphocyte counts between the head, body and tail of the epididymis were made using 
Wilcoxon signed rank tests. The significance of relationships between different measurements was assessed by Kendal1 rank correlation coefficients.

Comparison of 1ymphocyte counts in different tissues were made by unbalanced mixed model analysis of variance, using the program BMDP3V (Dixon, 1985). This allowed a fully efficient comparison in cases where some (but not all) donors had measurements made in both tissues. The significance levels obtained are for the pair-wise tests of each pair of tissues (organs). This analysis was carried out on the logarithm of lymphocyte counts in order to equalise variance in the different tissues.

\section{II.E.4 Photomicrography}

Stained sections were examined using a Carl Zeiss Jena optic microscope fitted with an automatic photomicrographic camera $(\mathrm{mF}$ AKS) (Figure II.4). Photomicrography was performed at the same time to illustrate the microscopical images. The focusing eye-piece $(x$ 6.3) is fitted with a framing graticule with two formal figures to indicate the image scale. These formal figures are sized so that the image section reliably fits into a $24 \mathrm{~mm} \times 36 \mathrm{~mm}$ format. This eye-piece was used in combination with high power objective lenses $\mathrm{x} 12.5, \mathrm{x} 25$ and $\mathrm{x} 50$ to produce pictures at magnifications $\mathrm{x} 80, \mathrm{x} 160$ and $\mathrm{x} 320$ respectively.

Black and white photography was performed using Panchromatic $35 \mathrm{~mm}$ films (Kodak, technical pan film, Estar-AH Base). To increase the contrast between the brown-stained cells and the blue 


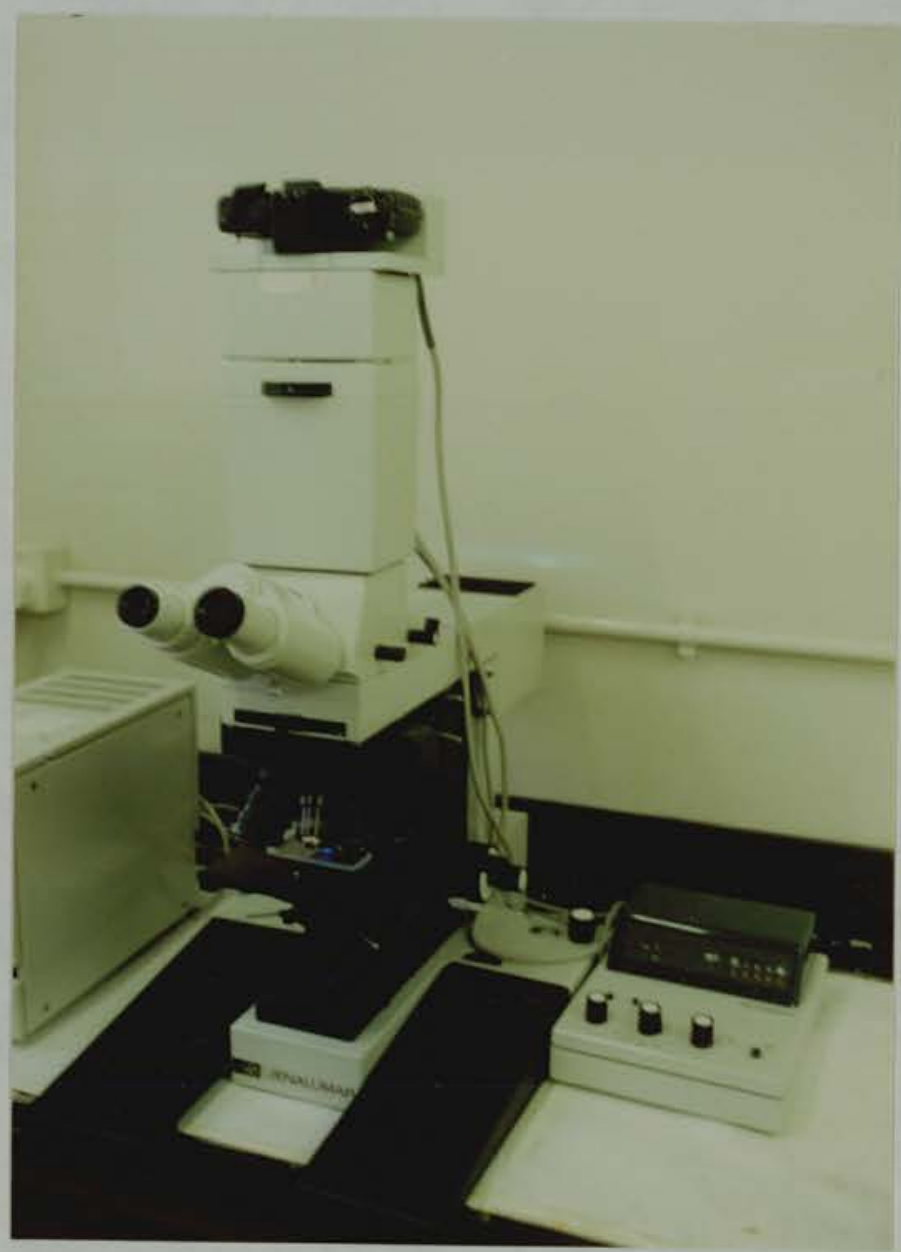

Figure II.4: A Carl Zeiss Jena optic microscope fitted with an automatic photomicrographic camera ( $\mathrm{mF}$ AKS) 
haematoxylin counter-stained areas, the exposure was made through a green coloured filter.

Coloured photography was performed using reversal films (Kodak EKa Chrome, Tungesten colour reversal film - 50) which provide colour transparencies. Colour prints were then produced from the positive transparencies.

Pictures were also obtained on colour negative films (Koda Color Gold 100) which normally are used to obtain colour prints. A blue coloured correcting filter was used. 
R E S U L T S 
CHAPTER III

OPTIMIZATION OF TECHNIQUES 


\section{III.A ROUTINE ASSESSMENT OF MONOCLONAL ANTIBODIES}

\section{III.A.1 Technical Quality of Immunoperoxidase Stained Sections and Smears}

\section{III.A.1.1 Staining patterns}

In the lymphoid tissues, two morphological staining patterns were clearly demonstrable when using specific monoclonal antibodies in the indirect immunoperoxidase technique. The first consisted of a distinctive membrane labelling: the cell periphery was clearly delineated by a dark brown margin contrasting with a pale cytoplasm. Such a ring pattern of staining was obtained with all the anti-lymphocyte antibodies. The second pattern was represented by a diffuse staining with an abolition of the nuclear counter-staining. This was obtained with the anti-macrophage, the anti-HLADR and the anti-interleukin 2 receptor antibodies. Diffuse intracytoplasmic staining was also demonstrated when cell smears were stained.

\section{III.A.1.2 The optimum working dilutions of the antibodies}

When interpreting dilution results, two criteria were usually under consideration: specific antigen staining and non specific background staining. The relation between the specific staining, background staining and the different dilutions of the antisera used is presented in Tables III.1 and III.2. Low 
TABLE III.1:

INTENSITY OF SPECIFIC AND BACKGROUND STAINING WITH THE INDIRECT IMMUNOPEROXIDASE TECHNIQUE USING DIFFERENT DILUTIONS OF ANTIHUMAN LEUCOCYTE MONOCLONAL ANTIBODIES

\begin{tabular}{|c|c|c|c|c|c|}
\hline \multirow{2}{*}{$\begin{array}{l}\text { Monoclonal } \\
\text { antibody }\end{array}$} & \multicolumn{5}{|c|}{ Intensity of Staining ${ }^{*}$} \\
\hline & & $1: 40$ & $1: 80$ & $1: 160$ & $1: 320$ \\
\hline Anti-human & specific & ++ & +++ & +++ & ++ \\
\hline leucocyte & background & ++ & + & - & - \\
\hline \multirow[t]{2}{*}{ Leu 4} & specific & +++ & +++ & +++ & ++ \\
\hline & background & ++ & + & - & - \\
\hline \multirow[t]{2}{*}{ Leu $3 a$} & specific & ++ & +++ & +++ & + \\
\hline & background & +++ & ++ & + & - \\
\hline \multirow[t]{2}{*}{ Leu $2 a$} & specific & +++ & +++ & +++ & ++ \\
\hline & background & ++ & - & - & - \\
\hline \multirow[t]{2}{*}{ Leu 14} & specific & +++ & +++ & +++ & ++ \\
\hline & background & ++ & + & - & - \\
\hline \multirow[t]{2}{*}{ Leu M3 } & specific & ++ & +++ & +++ & + \\
\hline & background & ++ & + & - & - \\
\hline \multirow[t]{2}{*}{ HL-ADR } & specific & +++ & +++ & ++ & +++ \\
\hline & background & +++ & ++ & - & - \\
\hline \multirow[t]{2}{*}{$1 \mathrm{~L} 2-\mathrm{r}$} & specific & +++ & +++ & +++ & ++ \\
\hline & background & ++ & + & - & - \\
\hline \multirow[t]{2}{*}{ Leu $11 \mathrm{~b}$} & specific & +++ & +++ & +++ & ++ \\
\hline & background & +++ & + & - & - \\
\hline \multirow[t]{2}{*}{ Leu 7} & specific & +++ & +++ & +++ & ++ \\
\hline & background & +++ & + & - & - \\
\hline
\end{tabular}

* Graded as intense +++; moderate ++; weak +; negative. 


\section{TABLE III.2:}

INTENSITY OF STAINING* WITH INDIRECT IMMUNOPEROXIDASE METHOD USING DIFFERENT DILUTIONS OF PEROXIDASE CONJUGATED RABBIT-ANTIMOUSE (RAM) ANTIBODY

\begin{tabular}{|c|c|c|c|}
\hline $\begin{array}{l}\text { First antibody } \\
\text { dilution } \mathbf{x}\end{array}$ & $\begin{array}{l}\text { RAM } \\
\text { dilution }\end{array}$ & $\begin{array}{l}\text { Specific } \\
\text { staining }\end{array}$ & $\begin{array}{l}\text { Background } \\
\text { staining }\end{array}$ \\
\hline $1: 40$ & $\begin{array}{l}1: 25 \\
1: 50 \\
1: 100\end{array}$ & $\begin{array}{l}++ \\
++ \\
+\end{array}$ & $\begin{array}{l}+++ \\
++ \\
+\end{array}$ \\
\hline $1: 80$ & $\begin{array}{l}1: 25 \\
1: 50 \\
1: 100\end{array}$ & $\begin{array}{l}++ \\
+++ \\
+\end{array}$ & $\begin{array}{l}++ \\
+ \\
-\end{array}$ \\
\hline $1: 160$ & $\begin{array}{l}1: 25 \\
1: 50 \\
1: 100\end{array}$ & $\begin{array}{l}++ \\
+++ \\
+\end{array}$ & $\begin{array}{l}+ \\
- \\
-\end{array}$ \\
\hline $1: 320$ & $\begin{array}{l}1: 25 \\
1: 50 \\
1: 100\end{array}$ & $\begin{array}{l}+ \\
++ \\
+\end{array}$ & $\begin{array}{l}+ \\
- \\
-\end{array}$ \\
\hline
\end{tabular}

\footnotetext{
Notes: * Graded as +++ intense; ++ moderate; + weak; - negative. $\mathrm{x}$ Anti-leu 2a was used as first antibody.
} 
dilutions $(1: 40)$ of the primary antisera of the Leu series gave the greatest background staining and specific staining began to diminish with high antisera dilutions (1:320). The maximum intensity, clarity and uniformity of specific staining with absent or minimal background staining were obtained with 1:160 dilutions. This dilution was selected as the optimum working dilution for the Becton Dickinson reagents.

The Coulter clone monoclonal antibodies T6, T11, T3, T4, T8 and B1 gave similar patterns of staining to that of the Leu series when used diluted 1:40 as recommended by the manufacturer. However the Leu series, being used at higher dilutions, 1:160, gave slightly better results with respect to sensitivity, intensity of the specific staining and lower background staining.

In general the strongest staining was obtained with the anti-Leu 2 a and T8 monoclonal antibodies which define the suppressor/cytotoxic T-cell subset. Specific staining for the helper/inducer T-cell subset using either the anti-Leu $3 \mathrm{a}$ or T4 antibodies was less intense as compared to that of the suppressor/ cytotoxic subset. Using the anti-Leu $3 a+b$ antibody, staining of the helper T-cells became more intense.

\section{III.A.1.3 Specificity controls}

Control sections in which a buffer or normal mouse serum (a non-immune serum) was substituted for the primary antisera were consistently negative on staining. Replacement of the primary antisera with a prostatic acid phosphatase monoclonal antibody (unrelated antibody which has no specificity for any antigen in the 
lymphoid tissue sections), resulted in negative staining in the lymphoid tissue sections and positive staining in prostatic tissue sections. This confirmed the specificity of the method. Sections of normal lymphoid tissue (spleen, lymph node and tonsils) stained with both the Leu antisera and the Coulter clone antibodies (see later) confirming the reactivity of these antibodies.

\section{III.A.1.4 Background staining}

Non specific staining was seen in the germinal centres of the lymphoid follicles, as weak intracellular staining, the smooth muscles of blood vessels and in the red pulp areas of the spleen. This staining occurred in the control sections treated with $\mathrm{DAB} / \mathrm{H}_{2} \mathrm{O}_{2}$ substrate alone and was therefore regarded an endogenous peroxidase staining. This was overcome by preincubating sections with $0.3 \%$ $\mathrm{H}_{2} \mathrm{O}_{2}$ in methanol for $10-15$ minutes.

It should be stressed that background staining was absent or very minimal in the negative control sections of the main tissues of the study, i.e. tissues of the genito-urinary organs, as endogenous peroxidase activity was not a problem in such tissues. In addition, the use of higher concentration salt in the buffer solution, normal rabbit serum, in the diluent, and optimum antibody dilution resulted in absence of background staining. 


\section{III.B LYMPHOCYTE SUBPOPULATIONS IN HUMAN LYMPHOID TISSUES}

\section{AND BLOOD}

In the lymphoid tissues, the reactivity of the different specific monoclonal antibodies was as follows:

\section{III.B.1 Lymph Nodes and Tonsils}

The staining patterns with the anti-B and anti-T antibodies in lymph nodes and tonsils were essentially identical.

The monoclonal antibodies anti-Leu 14 and $\mathrm{Bl}$, which define B-1ymphocytes, stained the majority of the cells in the primary follicles and in the mantle zones of the secondary follicles (follicles with germinal centres). Cells in the germinal centres showed cytoplasmic as well as membrane staining. Very few scattered $14^{+}\left(\mathrm{BI}^{+}\right)$cells were seen in the interfollicular and paracortical areas (Figure III.1).

The Leu 4 monoclonal antibody, which is a pan T-cell marker, stained the majority of cells in the paracortical and interfollicular areas (Figure III.2). Leu $4^{+}$cells were also seen in the follicles and in the mantle zones. The endothelial cells of the sinusoids showed no staining. The majority of the Leu $4^{+}$cell population also reacted with the anti-Leu 3 a antibody (Figure III.3), which defines the helper/inducer T-cells. In contrast, the anti-Leu 2a antibody, which defines the suppressor/ cytotoxic T-cells, stained a minority of cells of the Leu $4^{+}$population in the 


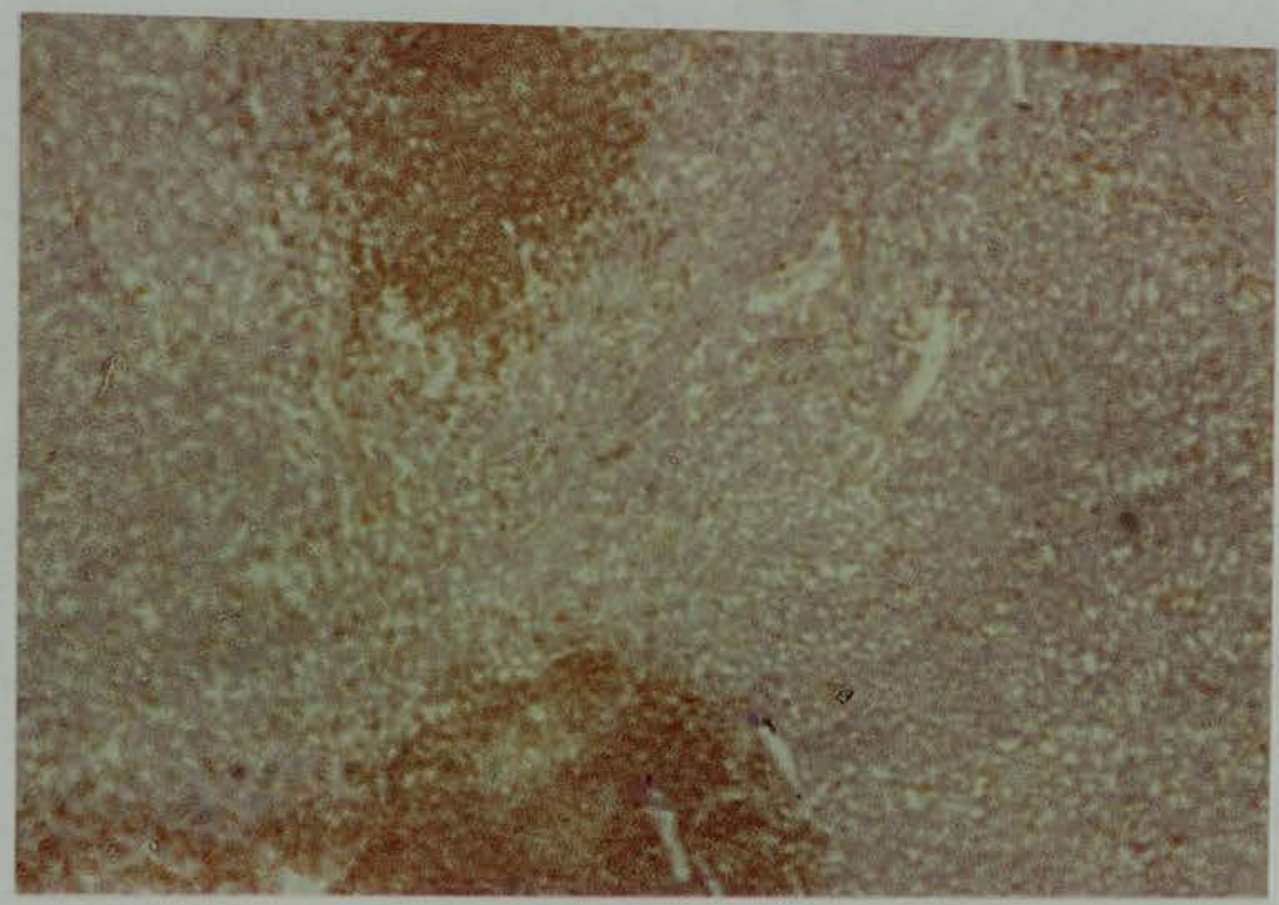

Figure III.1: Immunoperoxidase staining of a cryostat section of 1ymph node for B-1ymphocytes using the monoclonal antibody anti-Leu 14. (x 160) 


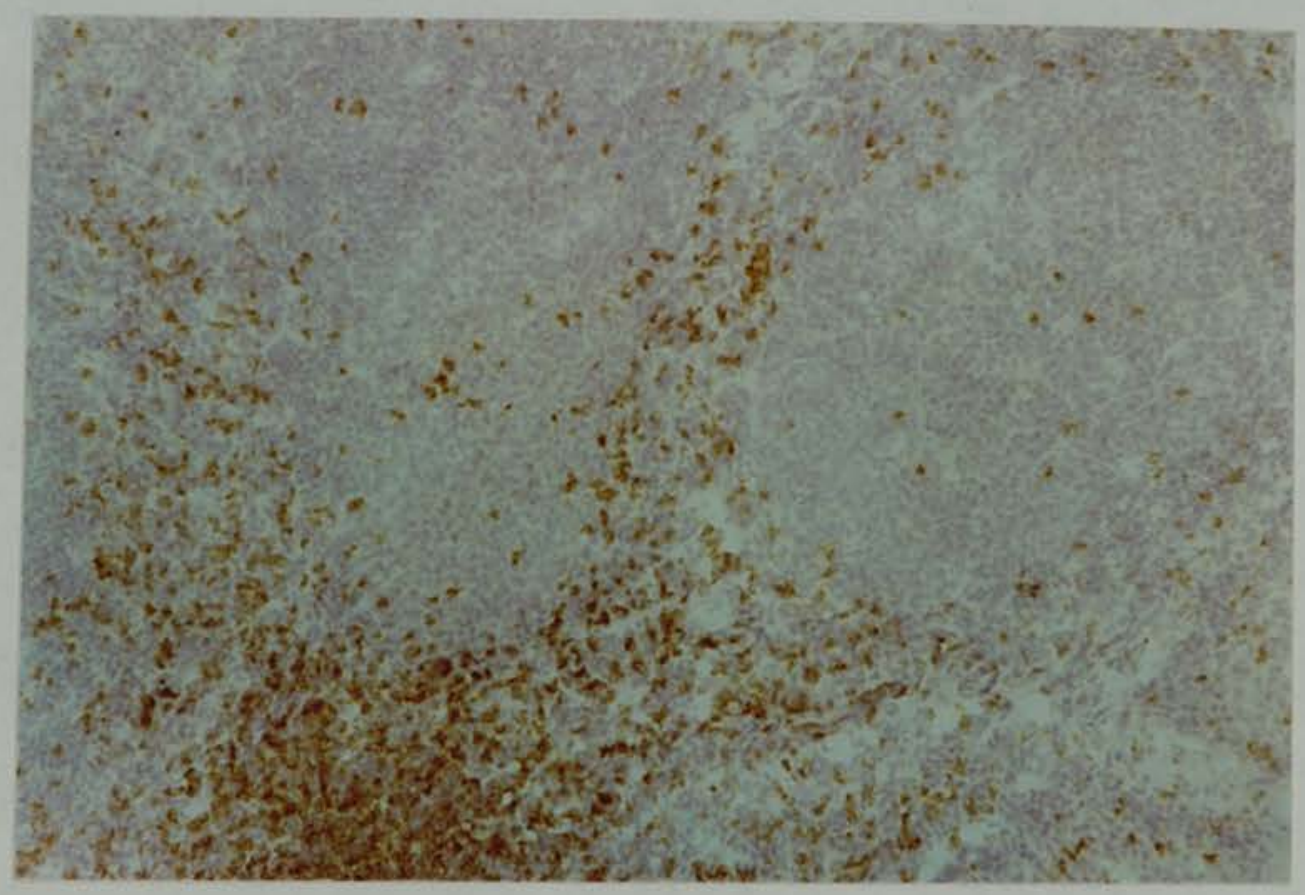

Figure III.2: Immunoperoxidase staining of a cryostat section of tonsil for T-1ymphocytes using the monoclonal antibody Leu 4. Note that the majority of stained cells are in the interfollicular areas. (x 160) 


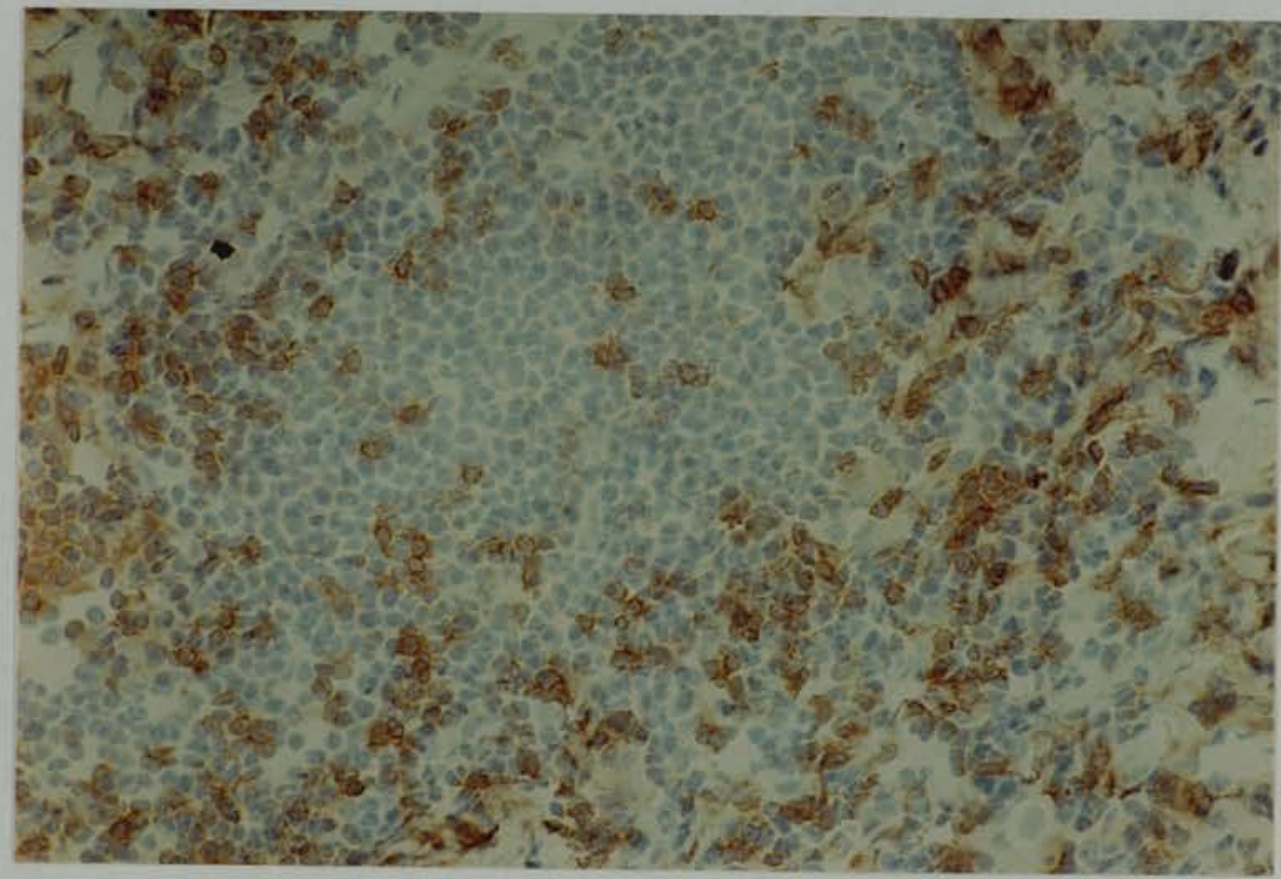

Figure III.3: Immunoperoxidase staining of a cryostat section of lymph node for helper/inducer T-cells using the monoclonal antibody Leu $3 \mathrm{a}$. Note the presence of Leu $3 \mathrm{a}^{+}$cells in the B-cell area. ( $\mathrm{x}$ 160) 
paracortical and interfollicular areas. Very few Leu $2 a^{+}$cells were detected within the follicles and the mantle zones. The monoclonal antibodies T3, T4, T8 gave similar patterns of staining to that of Leu 4, Leu $3 \mathrm{a}$ and Leu $2 \mathrm{a}$ antibodies.

The anti-1L2-r monoclonal antibody, which reacts with activated T-cells, stained a substantial number of cells in the paracortical and interfollicular regions, i.e. the T-cell areas. A few $1 \mathrm{~L}-2 \mathrm{r}^{+}$cells were also seen within the follicles, i.e. the B-cell areas. The positively stained cells showed specific membrane as well as cytoplasmic staining.

Cells reacting with the anti-Leu $M_{3}$ antibody, which defines monocytes/macrophages, were demonstrated in the interfollicular areas. The follicles showed no positive staining. Cells reacting with the Leu 7 and Leu 11 b antibodies, which define the natural killer (NK) cells, were more abundant and located mainly in the germinal centres of large secondary follicles (Figure III.4).

\section{III.B.2 Spleen}

The anti-Leu 14 antibody stained the majority of cells in the germinal centre, corona and the marginal zone of the follicles (white pulp), the B-cell area. Several cells in the periarteriolar lymphoid sheath (PALS) and in the red pulp were also stained. Leu 4 antibody stained almost all 1ymphocytes in the PALS, the T-cell areas (Fig. III.5), and scattered cells in the red pulp, and also a few cells in the follicles. A majority of these Leu $4^{+}$ 


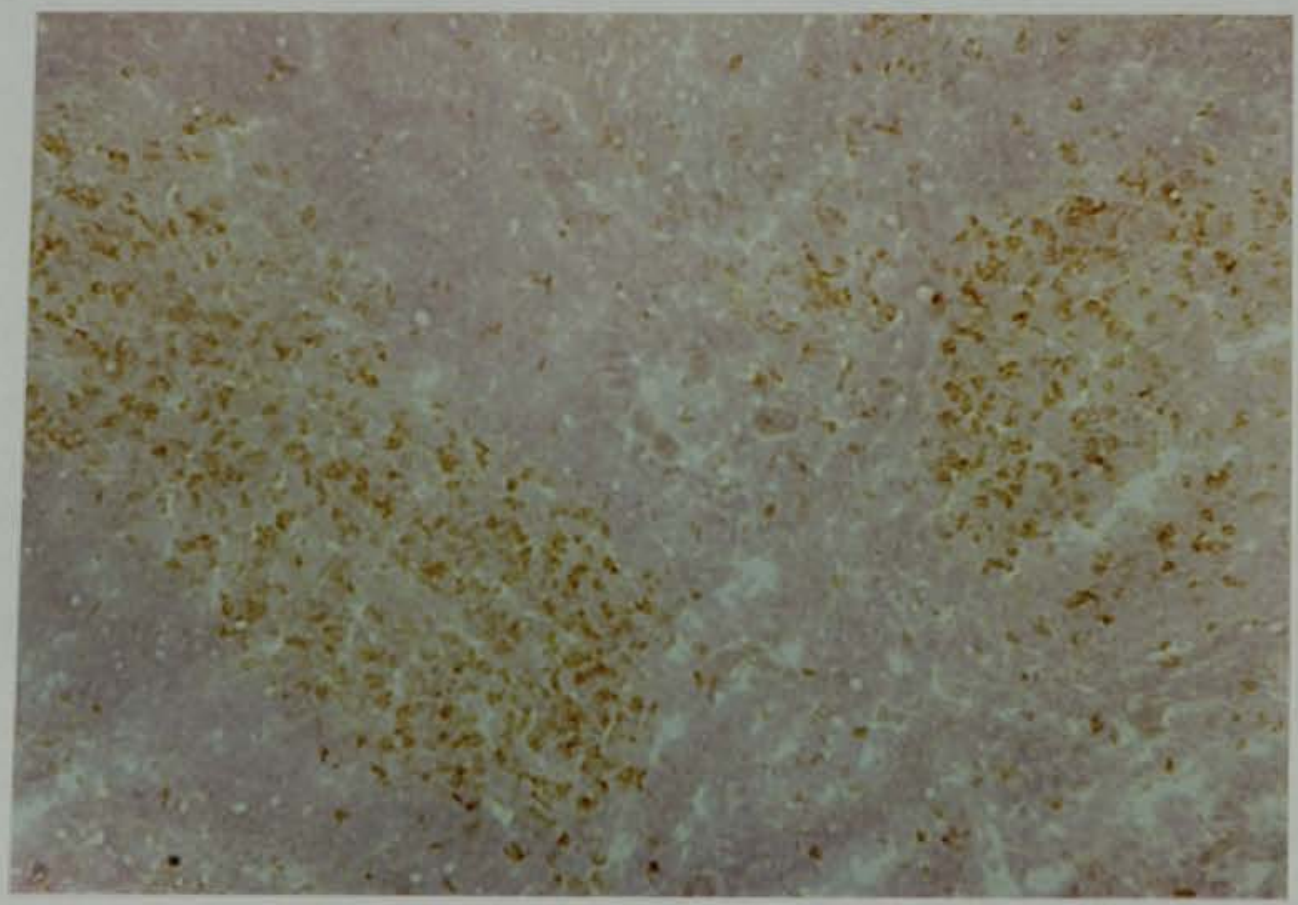

Figure III.4: Immunoperoxidase staining of a cryostat section of tonsil for NK-cells using the monoclonal antibody Leu $11 \mathrm{~b}$. Note that Leu $11 \mathrm{~b}^{+}$cells are located mainly in the germinal centres of secondary follicles. (x 160) 


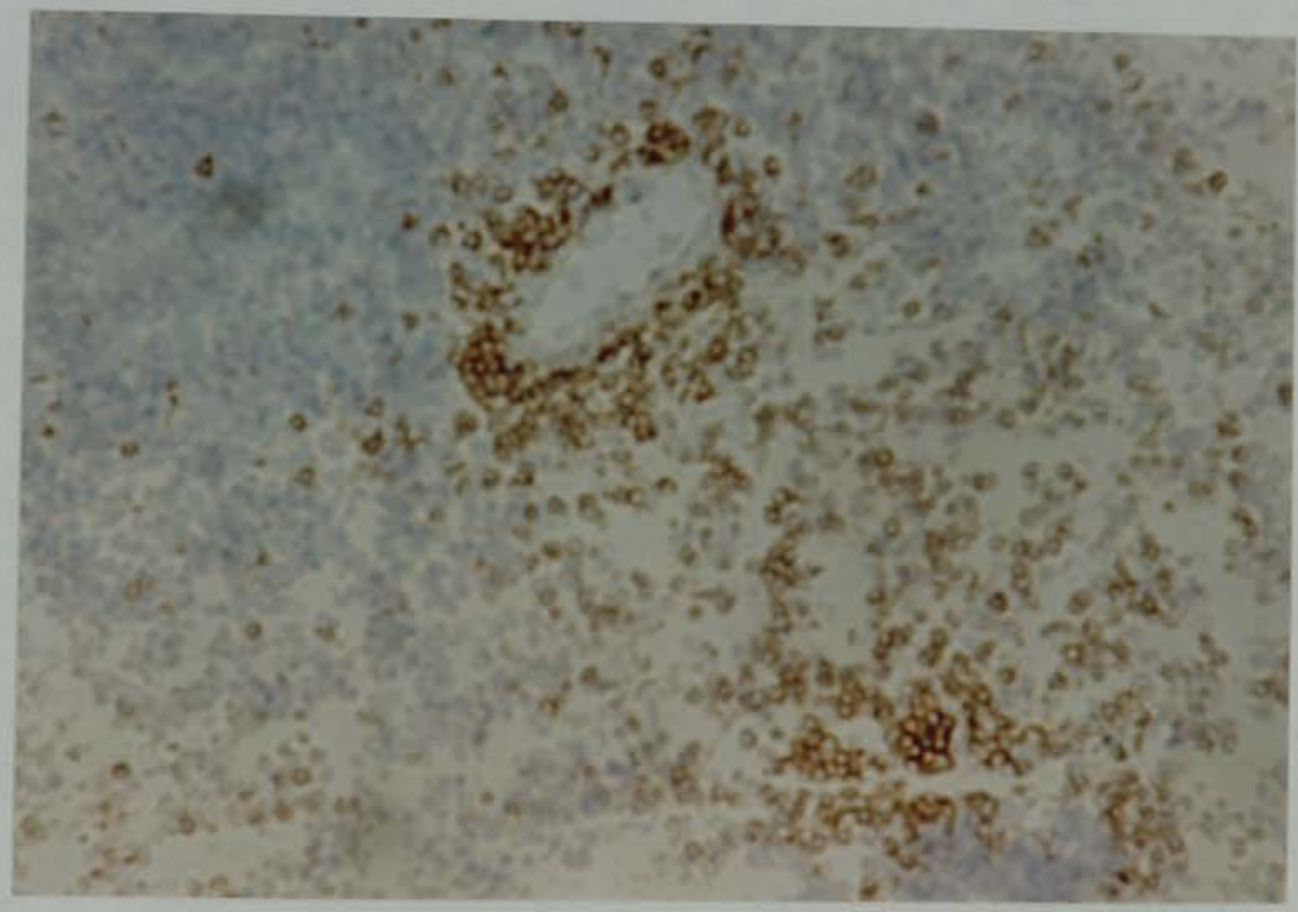

Figure III.5: Immunoperoxidase staining of a cryostat section of spleen for T-cells using the monoclonal antibody Leu 4. Note that most stained cells are located in the periarteriolar lymphoid sheath. (x 160) 
(T-cells) stained with the anti-Leu $3 a$ antibody whilst the minority stained with the anti-Leu $2 \mathrm{a}$ antibody. Almost all Leu $4^{+}$cells in the red pulp were Leu $2 \mathrm{a}^{+}$. A few cells in the PALS as well as in the red pulp reacted with the anti 1L2-r antibody which reacts with activated T-cells. Leu 7 and Leu 11b labelled a minority of cells in the follicles and in the red pulp. Leu $\mathrm{M}^{+}$(monocytes/ macrophages) were largely seen in the red pulp.

\section{III.B.3 Blood Leucocytes/Ejaculate Smears}

On staining of the cytocentrifuge smears of peripheral leucocytes, the positive cells were stained very strongly and contrasted clearly with the unstained cells. The positively staining cells showed specific membranes as well as cytoplasmic staining (Figure III.6). Negative control smears showed no staining, indicating that non specific staining did not occur. The morphological features as well as the staining properties of the cells preincubated with the latex microspheres were not altered. Polymorph nuclear leucocytes and monocytes showed specific staining after phagocytosis of the microspheres. The reactivity of the anti-lymphocyte antibodies with mononuclear cell smears is detailed in Table III.3.

The staining pattern seen in the ejaculate smears was similar to that seen in peripheral blood leucocyte smears (Figure III.7). Positively stained leucocytes were easily identified from other cells in the semen. 


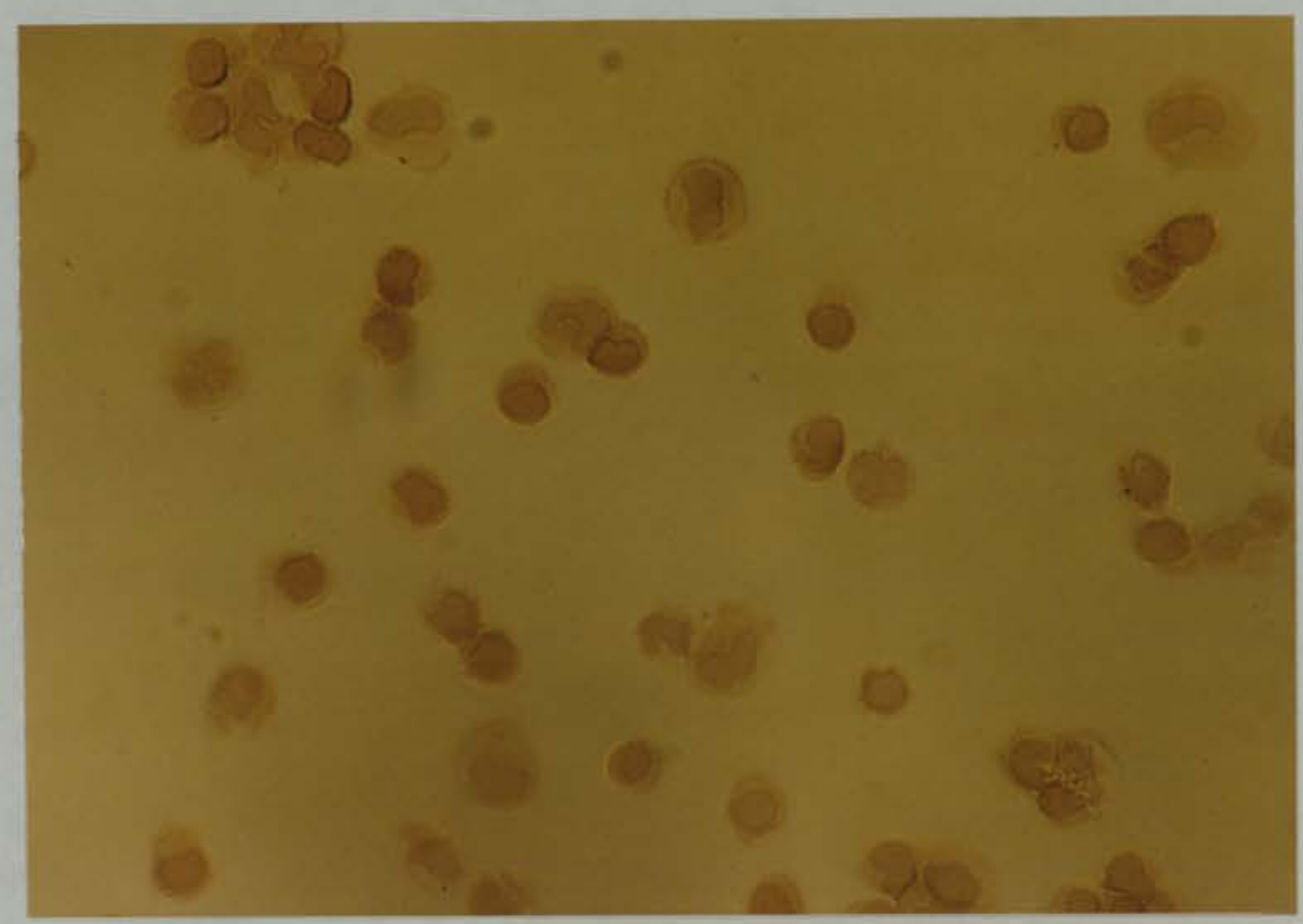

Figure III.6: Immunoperoxidase staining of a cytocentrifuge smear of human peripheral blood with the antihuman leucocyte monoclonal antibody.

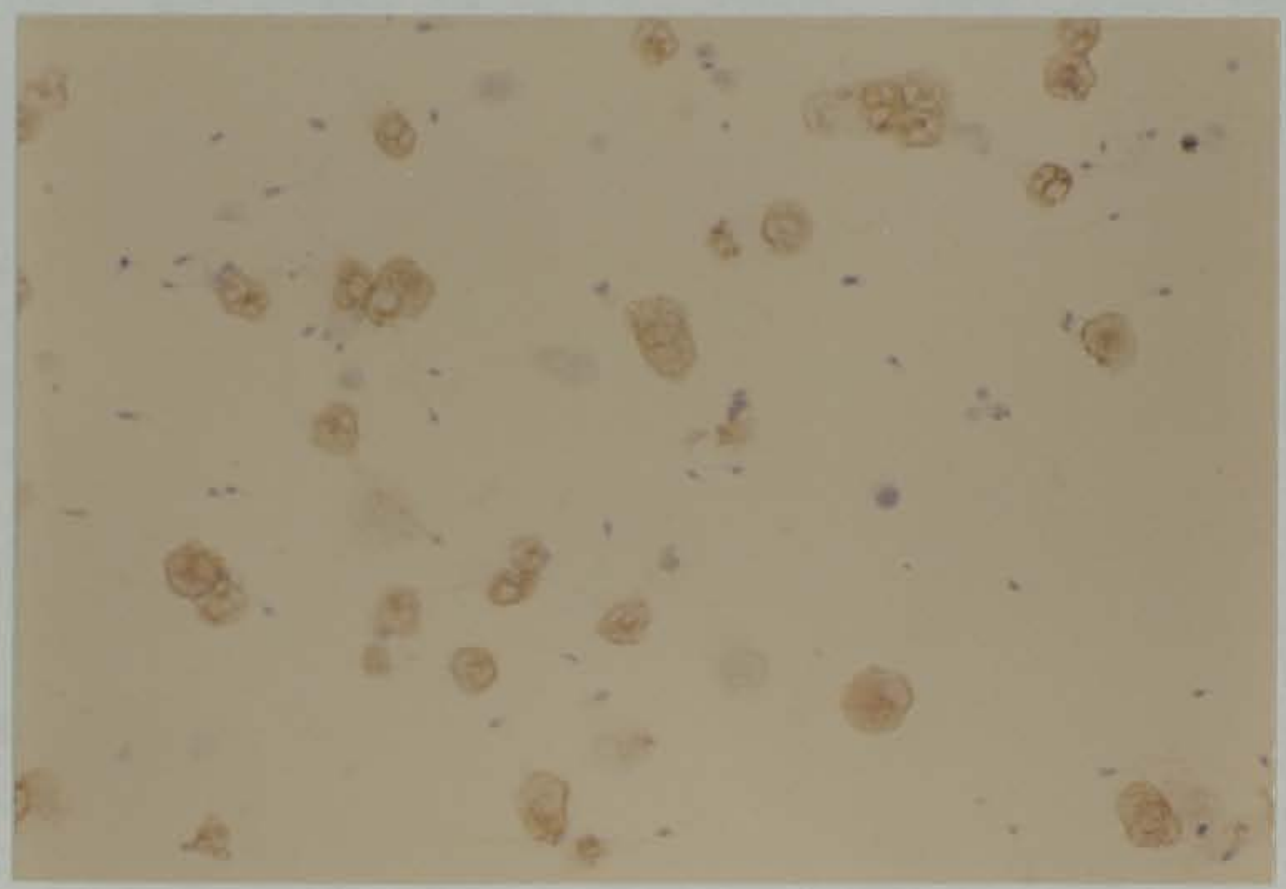

Figure III.7: Immunoperoxidase staining of a cytocentrifuge smear of human ejaculate with the antihuman leucocyte monoclonal antibody 
TABLE III.3:

PERCENTAGES OF LYMPHOCYTE SUBPOPULATIONS IN NORMAL PERIPHERAL BLOOD (CYTOCENTRIFUGE PREPARATION OF MONONUCLEAR CELLS)

\begin{tabular}{|c|c|c|c|c|c|}
\hline Donor & $\begin{array}{c}\text { Leu } 12^{+} \\
(\mathrm{B}-\mathrm{cell} \text { s })\end{array}$ & $\begin{array}{l}\text { Leu } 4^{+} \\
(\mathrm{T}-\mathrm{cell} \text { s) }\end{array}$ & $\begin{array}{l}\text { Leu } 3 \mathrm{a}^{+} \\
\text {( } \mathrm{T} \text { helper } / \\
\text { inducer) }\end{array}$ & $\begin{array}{c}\text { Leu } 2 \mathrm{a}^{+} \\
\text {( } \mathrm{T} \text { suppressor/ } \\
\text { cytotoxic) }\end{array}$ & $\begin{array}{l}\text { Leu } 3 a / \\
\text { Leu } 2 a\end{array}$ \\
\hline 1 & 17 & 71 & 50 & 29 & 1.72 \\
\hline 2 & 15 & 75 & 48 & 35 & 1.37 \\
\hline 3 & 20 & 80 & 55 & 27 & 2.03 \\
\hline 4 & 18 & 81 & 51 & 28 & 1.82 \\
\hline 5 & 25 & 65 & 45 & 30 & 1.50 \\
\hline 6 & 30 & 70 & 52 & 24 & 2.16 \\
\hline 7 & 12 & 73 & 47 & 28 & 1.67 \\
\hline 8 & 22 & 68 & 56 & 32 & 1.75 \\
\hline 9 & 16 & 70 & 49 & 33 & 1.48 \\
\hline 10 & 17 & 76 & 54 & 25 & 2.16 \\
\hline $\begin{array}{l}\text { A11 } \\
\text { Donors } \\
\text { (S.D.) }\end{array}$ & $19.2 \pm 5.26$ & $72.9 \pm 5.13$ & $50.7 \pm 3.5$ & \multicolumn{2}{|c|}{$1.766 \pm 0.279$} \\
\hline
\end{tabular}

Note: Results represent percentages of positive 1ymphocytes for 10 different specimens obtained from 10 healthy donors. 


\section{III.C DISCUSSION}

\section{III.C.1 Technical Aspects}

The use of the two layered indirect immunoperoxidase technique provided sufficient intensity of staining to reproducibly detect the lymphoreticular cells in the 1ymphoid tissue sections and in the cytocentrifuge smears of peripheral blood leucocytes. The method provided specific cell membrane staining of cells reacting with the specific monoclonal antibodies as revealed by well-formed dark brown rings outlining the membrane of the positively stained cells, e.g. lymphocytes. Specific cytoplasmic staining was also obtained when blood smears were stained. The use of proper controls was necessary for the validation of the immunoperoxidase staining technique. The presence of specific staining in the lymphoid tissue sections and the leucocyte smears (the positive controls) ensured not only that the immunoperoxidase technique was working but also that the method of tissue processing was satisfactory for the leucocyte antigens. It has been reported that the antigenicity of $\mathrm{T}$ - and $\mathrm{B}$-cell surface differentiation antigens defined by conventional or monoclonal antibodies is destroyed in fixed paraffin embedded tissues, but not in frozen sections (Falini and Taylor, 1983). Freezing of the tissues followed by sectioning on a cryostat offered the best preservation of immunoreactivity as indicated by the specific staining obtained. . The possible loss of tissue antigens during the tissue processing was prevented by proper tissue fixation in acetone prior to the 
staining procedure. It appears probable that these antigens, present in small amounts on the cell surface, are better preserved and detected in frozen sections, subjected to minimal fixation in acetone.

The negative controls were also useful in the evaluation of the validity of the immunoperoxidase staining technique. The absence of staining in the negative controls indicated that this immuno-enzymatic revealing technique is specific and is not giving rise to non specific labelling. Background staining was negligible or nearly absent and this is attributed to many factors:

1) Proper handling of the tissues in terms of freezing, sectioning and fixation. Similarly, proper preparation of leucocyte and ejaculate smears in respect of elimination of protein contamination and minimizing cell damage.

2) Blocking of the non specific reactivity by preincubating the sections/smears with non immune serum obtained from the same species as the second antibody, i.e. NRS. Non specific reactivity was further reduced by incorporating the normal rabbit serum into the primary antisera.

3) Blocking cross-reactivity with human immunoglobulin by adding normal human serum to the second antibody.

4) The use of high and proper dilutions of the primary antibodies.

5) Blocking of the endogenous peroxidase occasionally However this was not routinely done as this method meets with no complete success on frozen sections and can destroy the antigenicity of the cells. 
6) Increasing the intensity of the specific staining by incubating the slides in $2 \% \mathrm{CuSO}_{4}$ in normal saline.

The technique throughout this study has proved satisfactory for a wide variety of tissue antigens. Its advantages were that it is relatively quick to perform and that a single commercially available peroxidase conjugate was used.

\section{III.C.2 Lymphocyte Subpopulations in Lymphoid Tissues}

This study, utilizing specific monoclonal antibodies, with an indirect immunoperoxidase technique, shows that lymphoid organs are highly organised structures consisting of specific B- and T-cell compartments. In all three lymphoid organs - lymph nodes, tonsils and spleen - almost all cells of the follicles, whether primary or secondary, were B-lymphocytes. The follicles also showed T-lymphocyte specific staining, most of which were of the helper/inducer phenotype (Leu $3 \mathrm{a}^{+}$). NK-cells (Leu $7^{+}$) were also detected in the follicles. These Leu $3 a^{+}$and Leu $7^{+}$cells may play a regulatory role in maturation and differentiation of B-cells (James and Ritchie, 1984).

The distribution of $\mathrm{T}$-cell subsets was different in the three lymphoid organs. In the lymph nodes and tonsils both subsets of $\mathrm{T}$-cells were found in one compartment, the interfollicular areas. However, in the spleen the suppressor cytotoxic T-cells (Leu $2 \mathrm{a}^{+}$) were localized mainly in the red pulp, whereas the helper/inducer T-cells (Leu $3 \mathrm{a}^{+}$) were present predominantly in the PALS. In general, the results of this study are in agreement with the 
findings in previous reports of normal human lymph nodes, tonsils and spleen (Poppema et al., 1981; Ritchie et al., 1983; Timens and Poppema, 1985).

\section{III.C.3 Leucocytes/Ejaculate Smears}

Leucocyte smears and ejaculate/leucocyte smears showed a clear distinction between positive and negative cells and there was no background labelling of the antigen negative cells. Positive cells stained an intense brown which contrasted clearly with the haematoxylin counter-stain. The simplicity of the immunocytochemical technique used in this study, and the clarity with which it identifies different leucocyte cell populations in both leucocytes and ejaculate smears, suggests that it is an ideal means of identifying leucocyte subpopulations in human ejaculate (see Chapter VI).

The results of our study on mononuclear cells smears confirm the wide variation in the proportion of $\mathrm{T}-$ and $\mathrm{B}-\mathrm{cell}$ s in the blood of normal subjects. The results we obtained using the immunoperoxidase technique are in agreement with those obtained by Erber et al. (1984) and Hui and Lawton (1984). 
CHAPTER IV

LYMPHOCYTE SUBSETS AND MACROPHAGES IN THE TISSUES OF THE

MALE REPRODUCTIVE TRACT 
IV.A THE TESTIS

\section{IV.A.1 Normal Testicular Tissues}

\section{IV.A.1.1 Testicular histology}

Haematoxylin and eosin staining of each of the specimens from the eight cadaver donors showed intact germinal epithelium in a11 the seminiferous tubules with active meiotic activity throughout. Mature spermatozoa were identified in the majority of the tubules. The Leydig cells were unusually represented. No inflammatory changes and no vascular abnormalities were seen.

\section{IV.A.1.2 Immunohistology}

The use of the indirect immunoperoxidase technique provided sufficient intensity of staining to allow detection of 1ymphoreticular cells in frozen tissue sections. The reactivity of the primary monoclonal antibodies used in this study with sections of human spleen and 1ymph nodes (the positive control) was confirmed as similar to that reported in the previous chapter. Negative control sections showed no staining.

\section{IV.A.1.2.1 Anti-1eucocyte reactive cells}

Cells reacting with the pan anti-leucocyte (HLe-1) monoclonal antibody were consistently detected in both the 
peripheral testis and the rete testis. This antibody reacts with all human leucocytes in peripheral blood, spleen and tonsil, all thymocytes, infiltrating leucocytes in other tissues, B, T and myeloid cell lines (Beverley, 1980). These positively labelled cells were further characterised using monoclonal antibodies specific for lymphocyte subsets and tissue macrophages.

\section{IV.A.1.2.2 Lymphocytes}

In the normal peripheral testicular tissues none of the antilymphocyte monoclonal antibodies used gave a detectable reaction indicating absence of lymphocytes in these tissues. In marked contrast T-1ymphocytes were visualised throughout the rete testis with the anti-Leu 4 monoclonal antibody which is regarded as a pan T-cell marker. These T-1ymphocytes were located both within the lining epithelium of the tubules of the rete testis and within the stromal connective tissue of the rete testis (Figures IV.la and b). Studies with subset specific antisera revealed differences in distribution of the T-1ymphocyte subsets. Thus T-1ymphocytes of the suppressor/cytotoxic phenotype (Leu $2 \mathrm{a}^{+}$) were more abundant within the lining epithelium (Table IV.1) while the helper/inducer T-cells (Leu $3 \mathrm{a}^{+}$) predominated in the connective tissue stroma (Table IV.2). Only a few Leu $14^{+}$cells (B-cells) were demonstrated in the connective tissue stroma of the rete testis. 

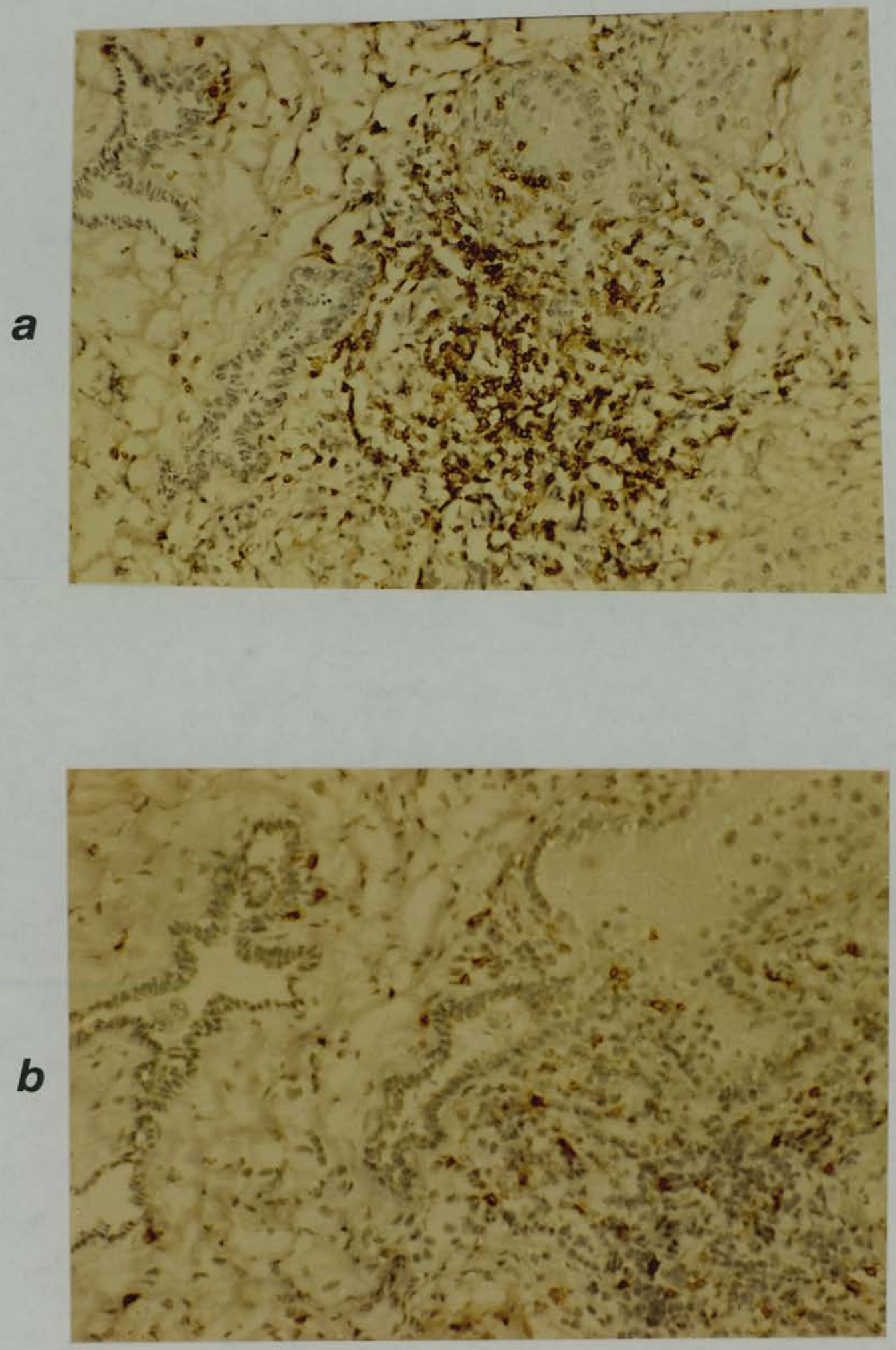

Figure IV.1: Immunoperoxidase staining of cryostat sections of rete testis for (a) suppressor/cytotoxic T-cells and (b) helper/inducer T-cells using the monoclonal antibodies Leu $2 \mathrm{a}$ and Leu $3 \mathrm{a}$ (x 160) 
TABLE IV.1:

REACTIVITY OF MONOCLONAL ANTIBODIES SPECIFIC FOR T-CELLS WITHIN THE EPITHELIUM OF THE RETE TESTIS

\begin{tabular}{llccl}
\hline Deu $4^{+}$cells & $\begin{array}{c}\text { Leu } 2 \mathrm{a}^{+} \text {cells } \\
\text { (T suppressor/ } \\
\text { cytotoxic) }\end{array}$ & $\begin{array}{c}\text { Leu } 3 \mathrm{a}^{+} \text {cells } \\
\text { ( } \mathrm{T} \text { helper/ } \\
\text { inducer) }\end{array}$ & $\begin{array}{l}\text { Leu 2a/ } \\
\text { Leu } 3 \mathrm{a}\end{array}$ \\
\hline
\end{tabular}

Mean counts of intraepithelial 1ymphocytes/100 epithelial cells

$\begin{array}{lllll}1 & 6.6 \pm 2.1 & 4.8 \pm 1.2 & 2.7 \pm 1.0 & 1.77 \\ 2 & 5.9 \pm 1.6 & 4.6 \pm 2.3 & 1.7 \pm 1.1 & 2.7 \\ 3 & 8.6 \pm 2.2 & 6.8 \pm 1.4 & 2.2 \pm 0.9 & 3.09 \\ 4 & 8.6 \pm 2.3 & 5.9 \pm 2.0 & 1.7 \pm 1.3 & 3.47 \\ 5 & 6.3 \pm 1.7 & 3.1 \pm 1.3 & 1.06 \pm 0.7 & 3.1 \\ 6 & 5.4 \pm 3.2 & 4.6 \pm 1.8 & 1.1 \pm 0.8 & 4.18 \\ 7 & 5.7 \pm 2.3 & 3.8 \pm 1.9 & 0.8 \pm 0.5 & 4.75 \\ 8 & 8.0 \pm 3.3 & 6.7 \pm 3.8 & 1.5 \pm 1.0 & 4.46\end{array}$
A11 donors
$6.9 \pm 1.3$
$5.0 \pm 1.3$
$1.6 \pm 0.6$
$3.44 \pm 0.99$

TABLE IV.2:

REACTIVITY OF MONOCLONAL ANTIBODIES SPECIFIC FOR T-CELLS WITHIN THE INTERTUBULAR CONNECTIVE TISSUE OF THE RETE TESTIS

\begin{tabular}{|c|c|c|c|c|}
\hline Donor & $\begin{array}{l}\text { Leu } 4^{+} \text {cells } \\
\text { (A11 T-cells) }\end{array}$ & $\begin{array}{l}\text { Leu } 2 \mathrm{a}^{+} \text {cells } \\
\text { (T suppressor } / \\
\text { cytotoxic) }\end{array}$ & $\begin{array}{c}\text { Leu } 3 \mathrm{a}^{+} \text {cells } \\
\text { (T helper/ } \\
\text { inducer) }\end{array}$ & $\begin{array}{ll}\text { Leu } 2 a / \\
\text { Leu } 3 a\end{array}$ \\
\hline
\end{tabular}

Average number of positive cells $/ 0.8 \mathrm{~mm}^{2}$ of intertubular connective tissue $(x$ 160) \pm S.D.

$\begin{array}{rrrrr}1 & 12.3 \pm 2.7 & 6.3 \pm 2.7 & 7.8 \pm 2.5 & 0.8 \\ 2 & 7.3 \pm 1.7 & 2.6 \pm 0.5 & 6.2 \pm 1.5 & 0.4 \\ 3 & 14.4 \pm 1.9 & 5.1 \pm 1.7 & 8.2 \pm 0.9 & 0.6 \\ 4 & 17.7 \pm 2.8 & 7.5 \pm 3.5 & 10.1 \pm 1.7 & 0.7 \\ 5 & 6.7 \pm 2.8 & 1.7 \pm 0.8 & 4.7 \pm 2.4 & 0.4 \\ 6 & 6.4 \pm 1.0 & 2.5 \pm 1.6 & 2.9 \pm 1.4 & 0.9 \\ 7 & 6.8 \pm 1.5 & 2.2 \pm 1.4 & 4.5 \pm 1.0 & 0.5 \\ 8 & 15.3 \pm 4.7 & 5.0 \pm 2.9 & 12.5 \pm 4.8 & 0.4\end{array}$
A11 donors
$10.8 \pm 4.6$
$4.1 \pm 2.1$
$7.1 \pm 3.1$
$0.6 \pm 0.2$ 


\section{IV.A.1.2.3 Macrophages}

Cells reacting with the anti-Leu M3 monoclonal antibody which is believed to bind specifically to monocytes/macrophages (Dimitriu-Bona et a1., 1983), were detected in large numbers in between the seminiferous tubules (Figure IV.2a). In order to substantiate this observation, staining was also performed with other monoclonal antibodies designated 3.9 and 24 known to bind to tissue macrophages and cells of interdigitating or dendritic appearance (Hogg and Selvendran, 1985; Hogg et al., 1986). These antisera also bound cells with a similar location to that of Leu $\mathrm{M3}^{+}$ cells (Figure IV.2b). These Leu M3 ${ }^{+}, 3.9^{+}$and $24^{+}$cells were largely arranged in a concentric fashion around the seminiferous tubules and in very close proximity to the outer aspect of their walls (the tunica propria). No positively stained cells were detected within the component cells of germinal epithelium. Other labelled cells were lying within the interstitial tissues, being abundant around the blood vessels. Labelled cells of this type were also seen in the rete testis: within the epithelium and within the connective tissue stroma.

The anti T-6 antibody which reacts with the epidermal Langerhan's cell gave no detectable reaction with normal testis. This suggests that the Leu $\mathrm{M}^{+}, 3.9^{+}$and $24^{+}$cells within the testis are not of the Langerhan's cell type. 

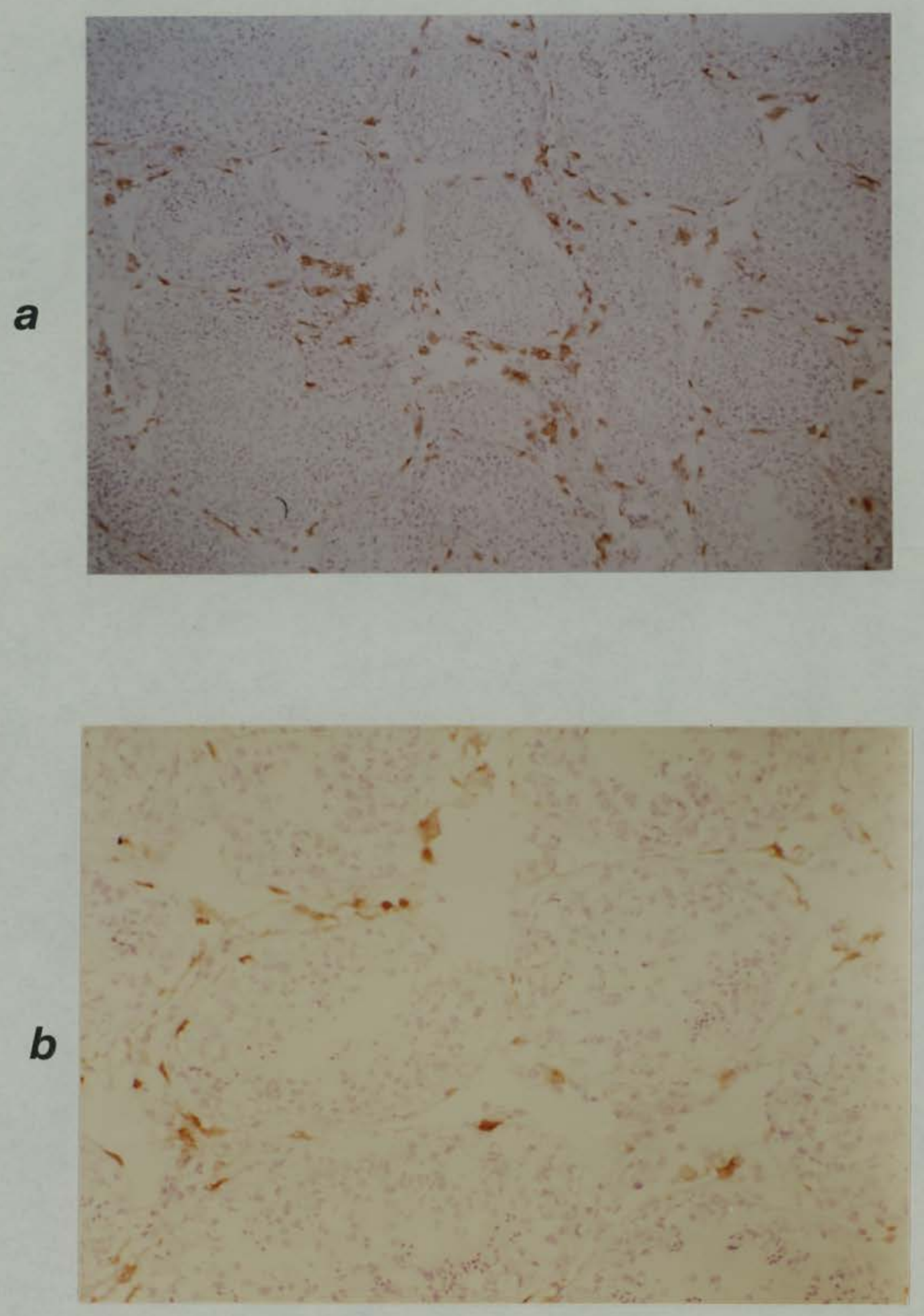

Figure IV.2: Immunoperoxidase staining of cryostat sections of normal testicular tissues with (a) Leu M3 and (b) 3.9 monoclonal antibodies which define monocytes/ macrophages (x 160) 


\section{IV.A.1.2.4 The anti-HLA-DR reactive cells}

Cells staining with the anti-HLA-DR monoclonal antibody exhibited a similar location and distribution to that of the Leu $\mathrm{M}^{+}, 3.9^{+}$and $24^{+}$cells (Figure IV.3). The staining pattern of the anti-HLA-DR antibody overlapped that of the antimacrophage antisera. This suggests that the majority of these cells are macrophages expressing HLA-DR antigens. Unlike the 1 ining epithelium of the seminiferous tubules (the germ cells and Sertoli cells) which was HLA-DR negative, the lining epithelium of the rete testis tubules was HLA-DR positive. The endothelial surface of the blood cells was also stained.

\section{IV.A.2 Testicular Biopsies from Infertile Men}

While no lymphocytes were detected in normal peripheral testicular tissues, 1ymphocytes were detected in all the testicular biopsies from infertile males (24/24).

In patients with oligozoospermia $(n=10)$, Leu $4^{+}$cells (T-1ymphocytes) were detected as single cells in the interstitial tissues between the seminiferous tubules. These T-cells were almost always of the suppressor/cytotoxic phenotype (Leu $2 \mathrm{a}^{+}$) and were few in number. None of these patients had detectable sperm-antibody in his serum or seminal plasma.

In patients with testicular obstruction ( $=14)$, intratesticular 1 ymphocytes were demonstrated in large numbers and they were essentially all Leu $4^{+}$cells (T-1ymphocytes). A few Leu $14^{+}$cells (B-lymphocytes) were seen. The lymphocytes were found in 


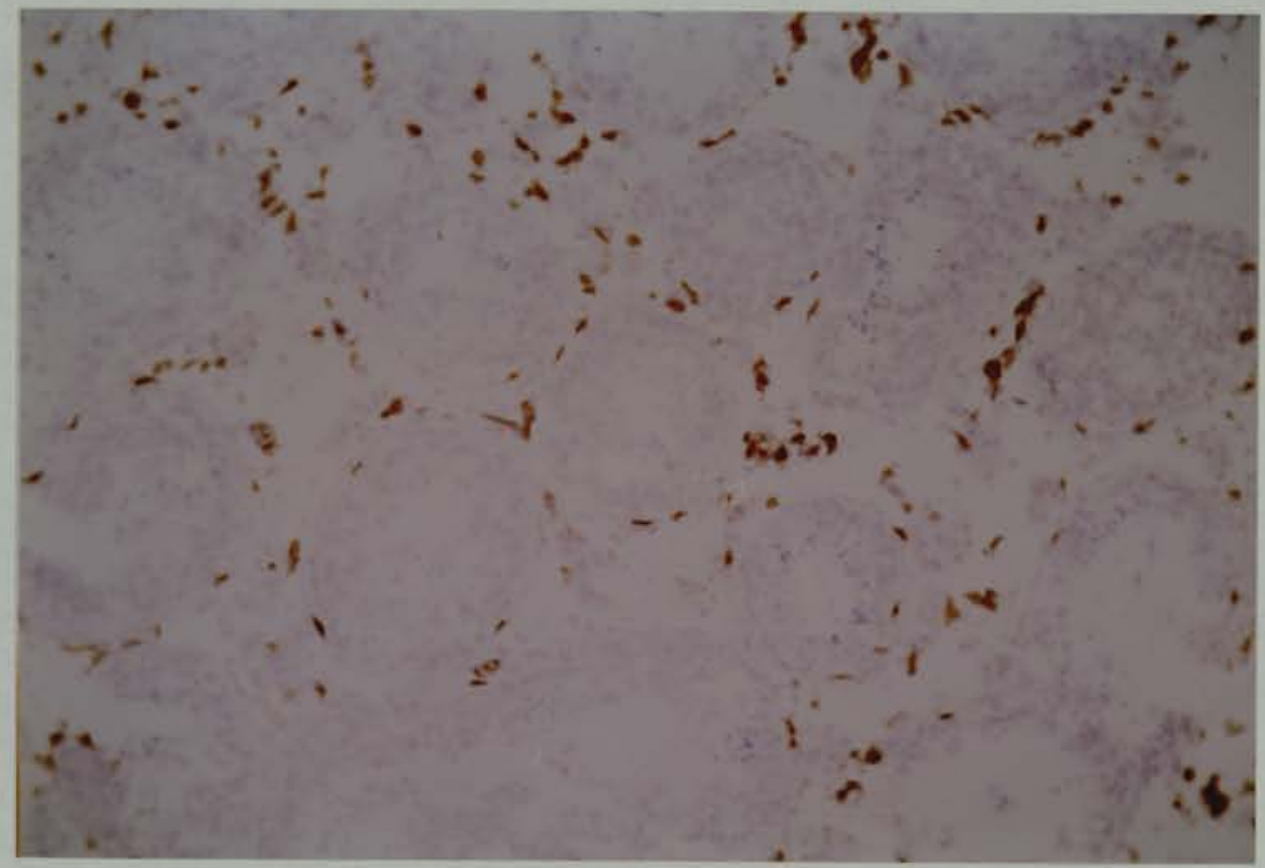

Figure IV.3: HLA-DR positive cells in normal testicular tissue.

Note that the staining pattern of the anti HLA-DR antibody overlapped completely that of the antimacrophage antibodies. (Cryostat section, immunoperoxidase, x 80). 
two locations. Isolated cells were noted between the fibrous tunica propria and the germinal epithelium of the seminiferous tubules (Figure IV.4a). No labelled cells were seen within the germinal epithelium. The other lymphocytes were arranged in small aggregates and follicle-like clusters within the intertubular connective tissue (Figure IV.4b).

In biopsies from patients with obstructive azoospermia ( $\mathrm{n}=$ 6), T-1ymphocytes of the suppressor/cytotoxic phenotype (Leu $2 \mathrm{a}^{+}$) predominated, making the average T-suppressor/cytotoxic to the T-helper/inducer ratio (Leu $2 \mathrm{a}^{+} /$Leu $3 \mathrm{a}^{+}$) approximately 4 to 1 (Table IV.3). The Leu $2 \mathrm{a}^{+} /$Leu $3 \mathrm{a}^{+}$ratios were significantly higher in these patients than in those with unilateral obstruction $(P<0.05)$ or vas reversal $(P<0.01)$. However the total number of Leu $4^{+}$and of Leu $2 \mathrm{a}^{+}$cells did not differ significantly among the three patient groups of testicular obstruction. Of these six patients with obstructive azoospermia, only one patient had sperm antibody detected at low titre $(1 / 8)$ in his serum.

In contrast, in the biopsies from vas reversal (post vasectomy) patients $(n=6)$ and patients with unilateral testicular obstruction, the helper/inducer T-cells (Leu $3 \mathrm{a}^{+}$) predominated. Levels of the Leu $3 a$ positive cells were significantly higher $(P<0.01)$ in these patients than in those with obstructive azoospermia (Figure IV.5) B-1ymphocytes (Leu $14^{+}$) and activated $\mathrm{T}-\mathrm{cel1s}\left(1 \mathrm{~L} 2-\mathrm{r}^{+}\right)$were also noted more frequent1y in comparison to the previous groups. All these patients had sperm antibody detected in high titres in their serum and semen (Tables IV.4 and 5). 

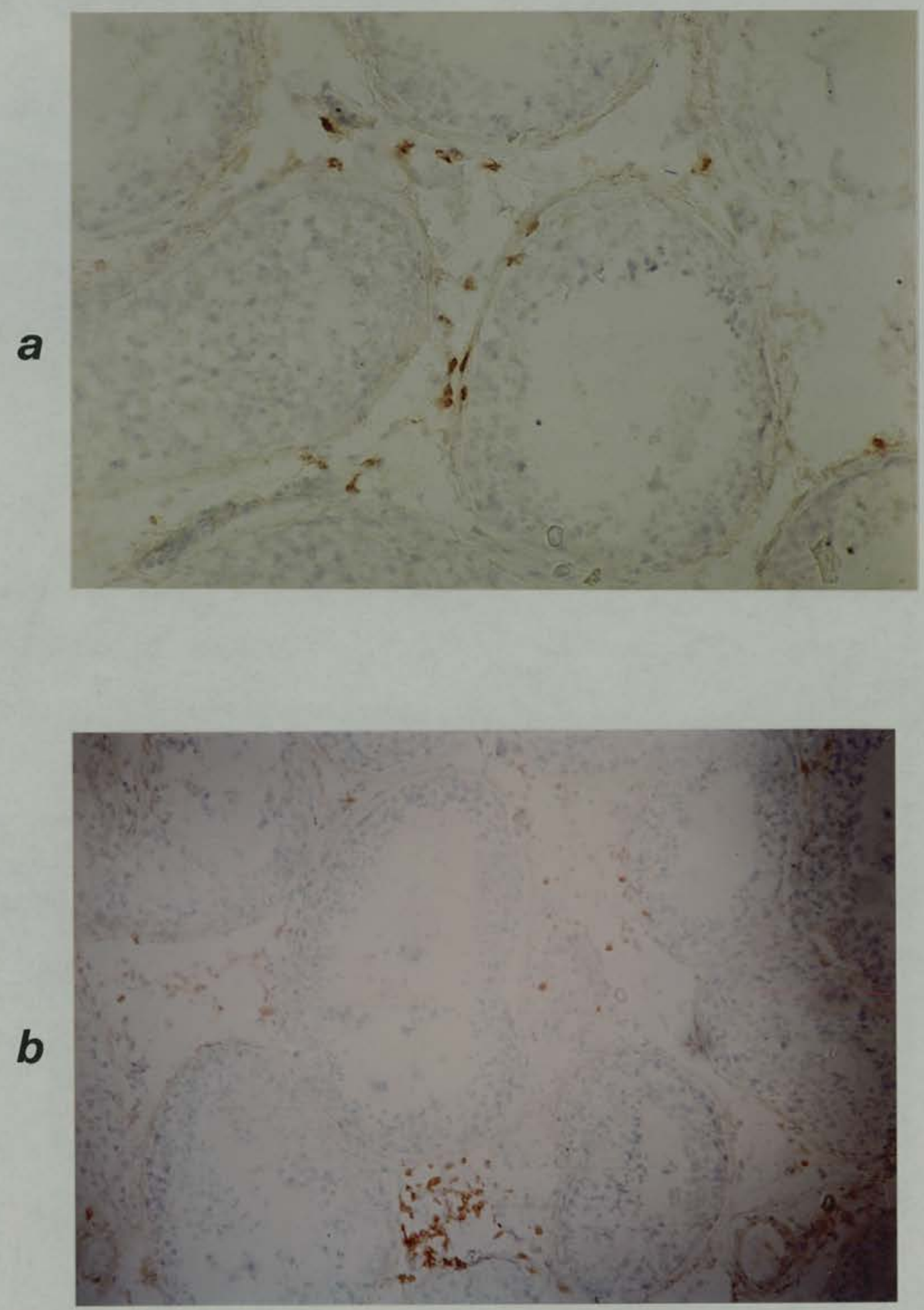

Figures IV.4: (a) Suppressor/cytotoxic T-cells (Leu $2 \mathrm{a}^{+}$) in a testicular biopsy from a patient with obstructive azoospermia; (b) helper/inducer T-cells (Leu $3 \mathrm{a}^{+}$) in a testicular biopsy from a vasectomy reversal patient.

(Cryostat sections, immunoperoxidase, $x$ 160) 


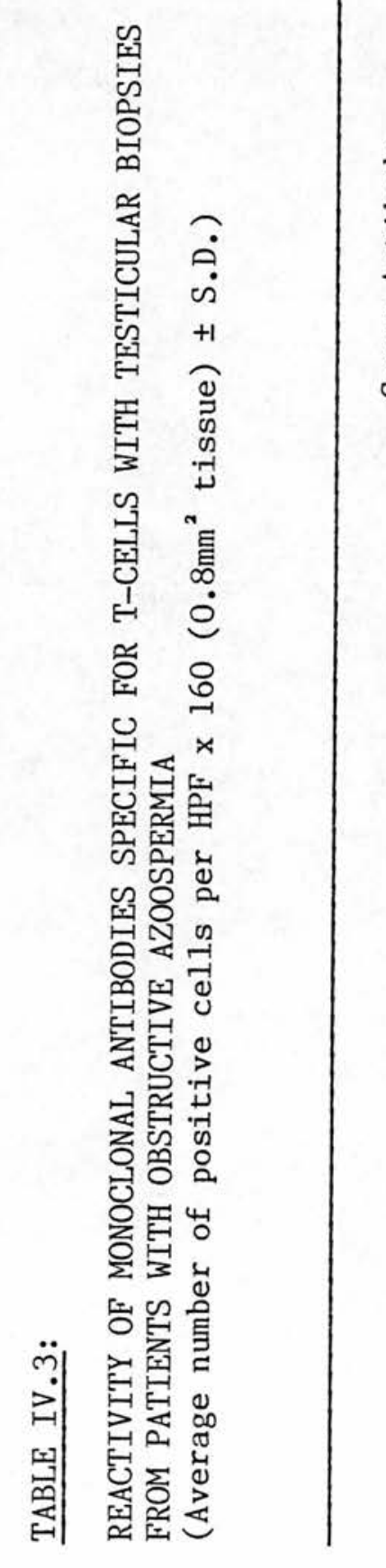

\begin{tabular}{|c|c|c|c|c|c|c|}
\hline 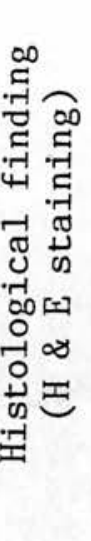 & 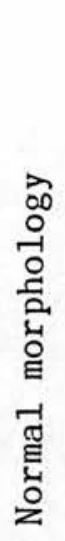 & 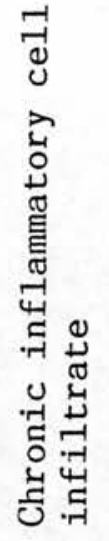 & 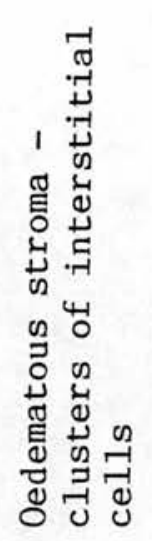 & 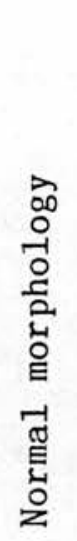 & 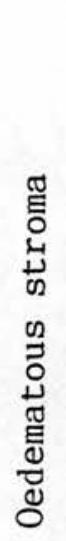 & 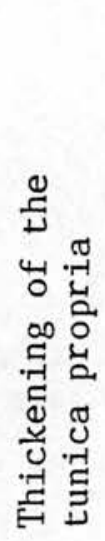 \\
\hline $\begin{array}{l}\text { む } \\
\text { ङ } \\
\text { ळ }\end{array}$ & 0 & 0 & 0 & 0 & 0 & 0 \\
\hline 疋 & 0 & 0 & 0 & 0 & $\infty$ & 0 \\
\hline త్త & $\stackrel{v}{\vartheta}$ & $\begin{array}{l}0 \\
\dot{j}\end{array}$ & $\stackrel{\sim}{\dot{j}}$ & $\begin{array}{l}\circ \\
\dot{j}\end{array}$ & $\stackrel{9}{\dot{m}}$ & $\stackrel{0}{\sim}$ \\
\hline 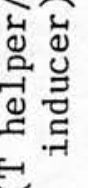 & $\begin{array}{l}0 \\
-\dot{1} \\
+1 \\
\infty \\
-\dot{-1}\end{array}$ & $\begin{array}{l}-\ddot{1} \\
\dot{+1} \\
\text { ஸे } \\
\dot{v}\end{array}$ & $\begin{array}{l}\stackrel{\leftrightarrow}{\dot{H}} \\
\stackrel{+1}{+1} \\
\dot{\sim}\end{array}$ & $\begin{array}{l}\text { ? } \\
\dot{+} \\
+1 \\
\text { m. }\end{array}$ & $\begin{array}{l}\stackrel{9}{0} \\
\stackrel{+1}{+} \\
\stackrel{-}{-1}\end{array}$ & $\begin{array}{l}\ddot{-} \\
\dot{+1} \\
\dot{0}\end{array}$ \\
\hline 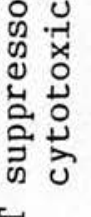 & $\begin{array}{l}\stackrel{\sim}{\sim} \\
\stackrel{+}{+} \\
\stackrel{\sim}{\rightleftharpoons}\end{array}$ & $\begin{array}{l}\underset{\sim}{\sim} \\
\underset{+}{+} \\
\stackrel{\sim}{\sim}\end{array}$ & $\begin{array}{l}\sim \\
\stackrel{+}{+1} \\
\infty \\
\infty \\
\infty\end{array}$ & $\begin{array}{l}\stackrel{0}{\dot{H}} \\
\dot{+} \\
\dot{\sim}\end{array}$ & $\begin{array}{l}0 \\
\ddot{+} \\
\dot{+1} \\
\dot{0}\end{array}$ & $\stackrel{\text { ? }}{\stackrel{+1}{+}}$ \\
\hline 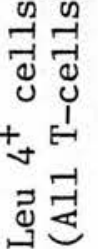 & $\begin{array}{l}\stackrel{+}{\sim} \\
\stackrel{+}{+} \\
\stackrel{\sim}{\sim} \\
\stackrel{-}{1}\end{array}$ & $\begin{array}{l}0 \\
\dot{\oplus} \\
+1 \\
\stackrel{+}{+} \\
\dot{y}\end{array}$ & 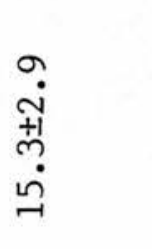 & $\begin{array}{l}\stackrel{?}{2} \\
\dot{+1} \\
\dot{0}\end{array}$ & $\begin{array}{l}\stackrel{9}{+-1} \\
\stackrel{+1}{a} \\
\dot{\sigma}\end{array}$ & 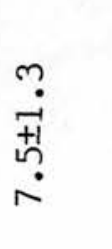 \\
\hline$\stackrel{\vec{\omega}}{\tilde{\omega}}$ & 㪯 & 弚 & 蛋 & ". & ซै & 孞 \\
\hline
\end{tabular}




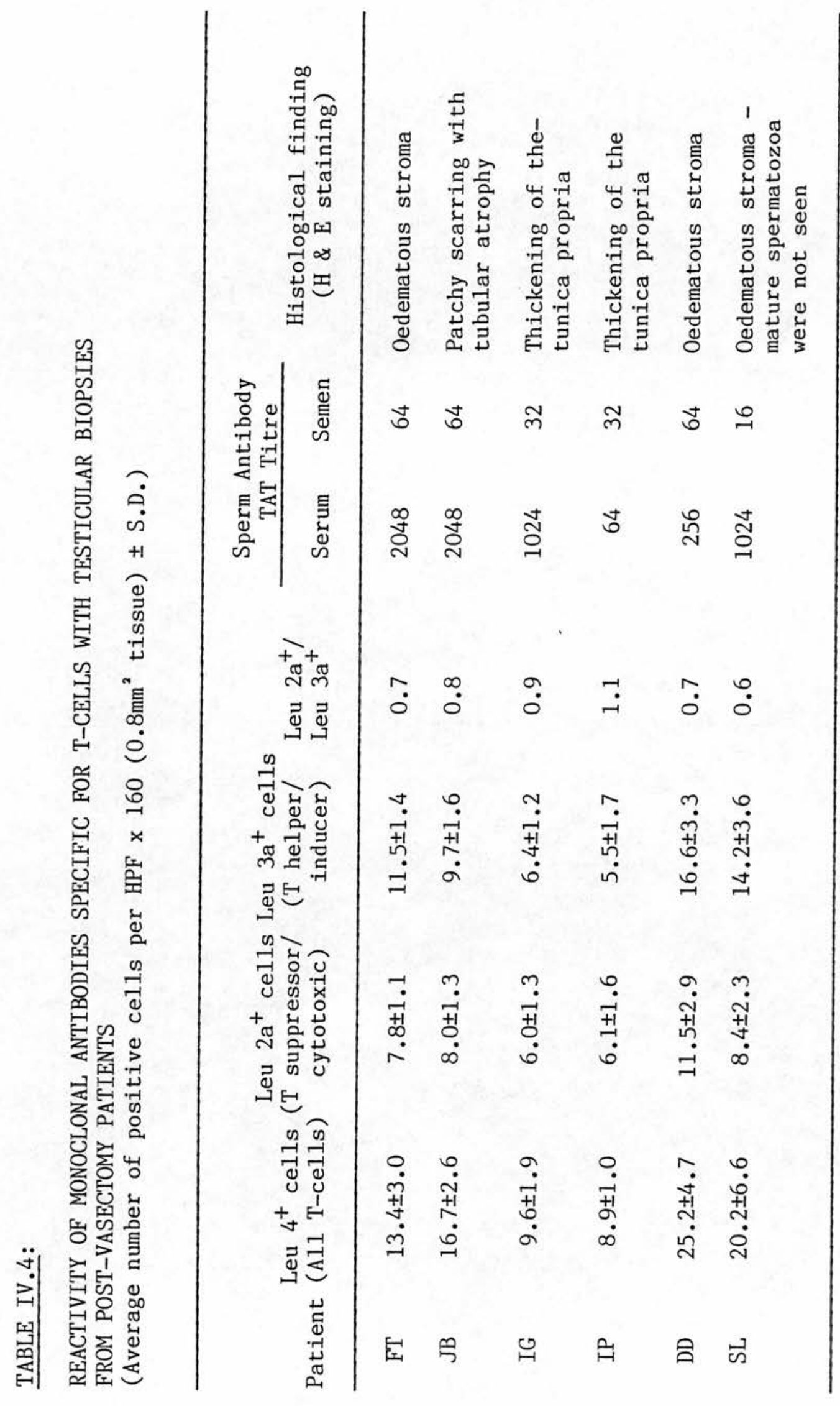




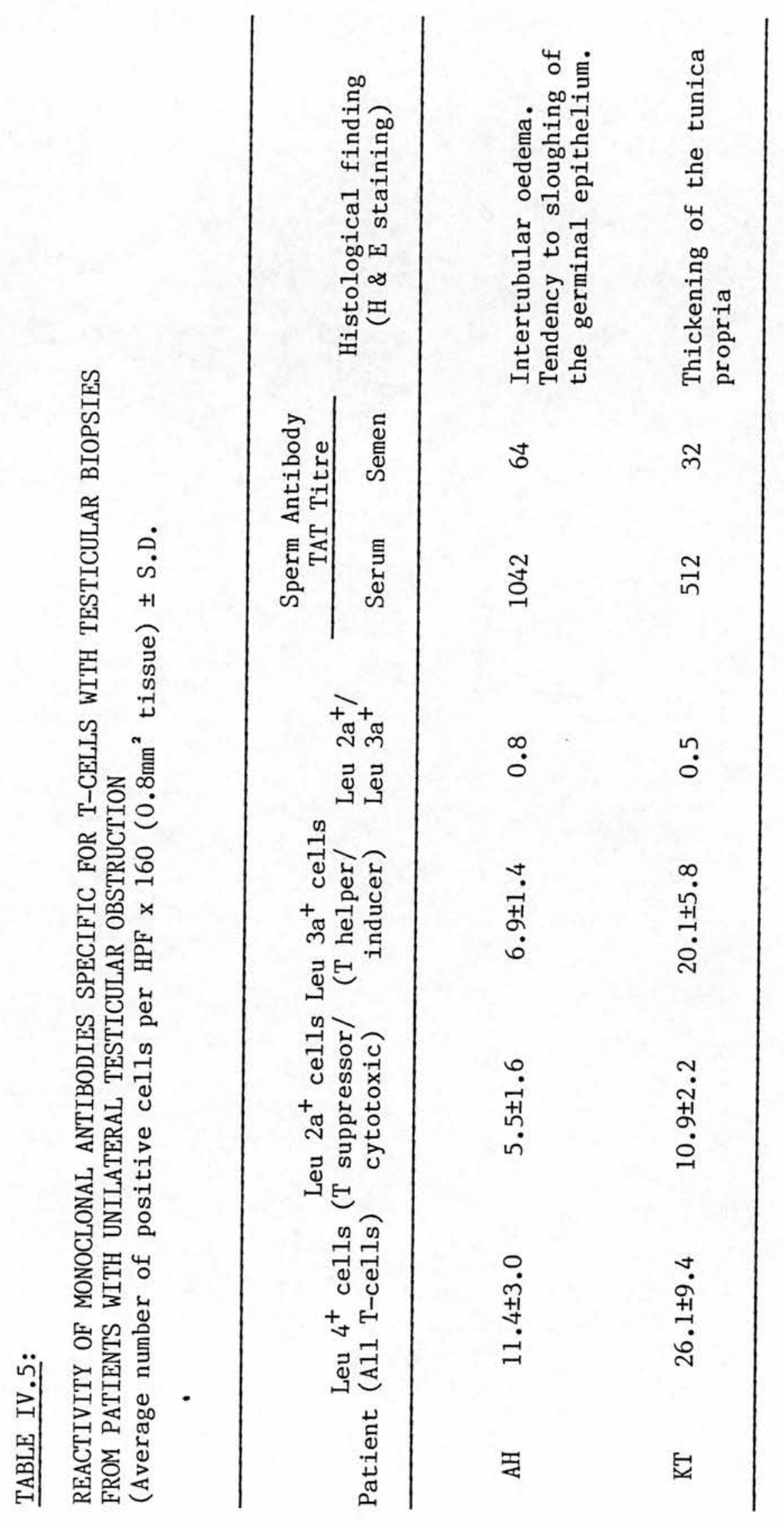


FIGURE IV 5

T-lymphocyte subsets in patients with testicular obstruction

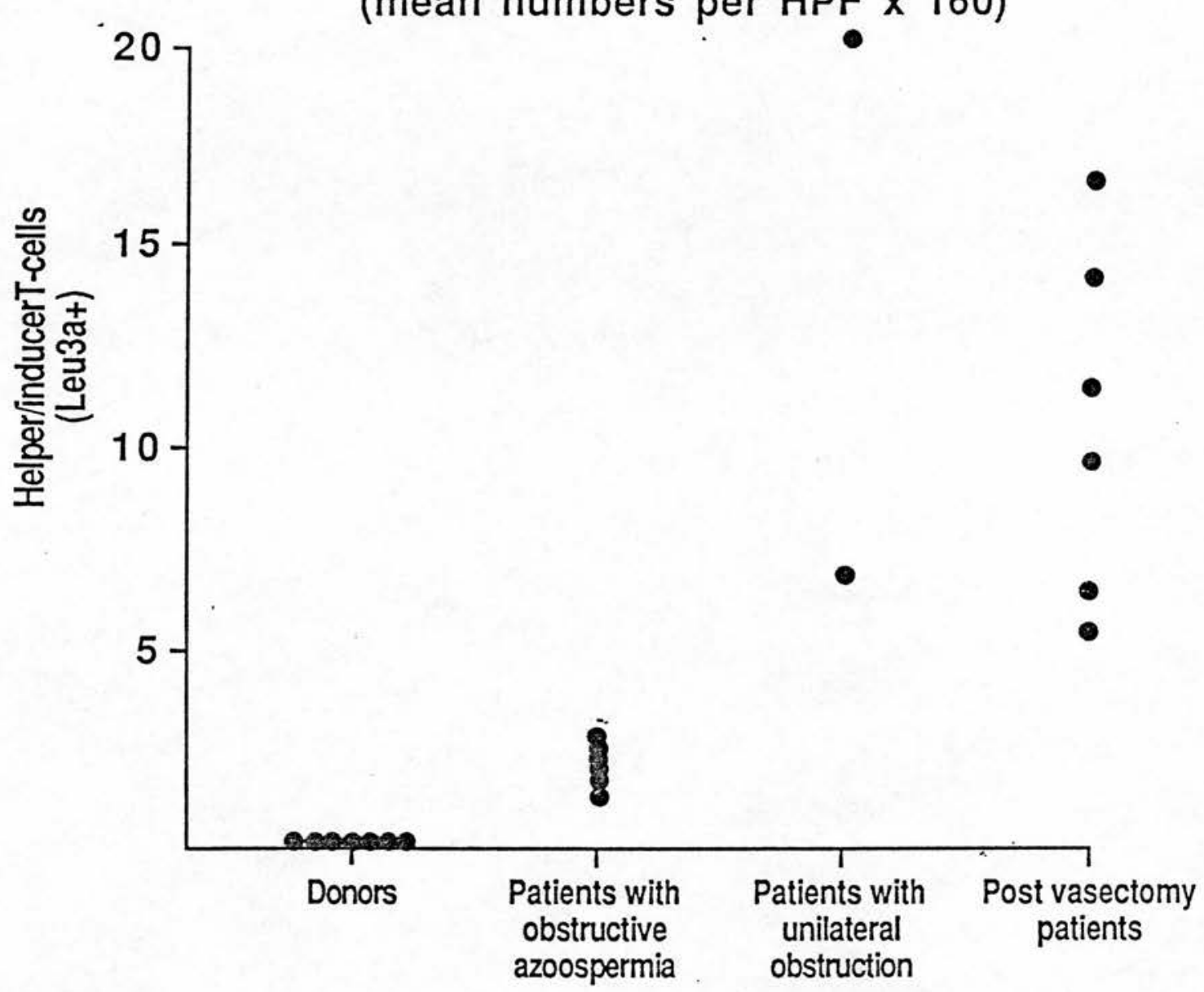
(mean numbers per HPF $x$ 160)

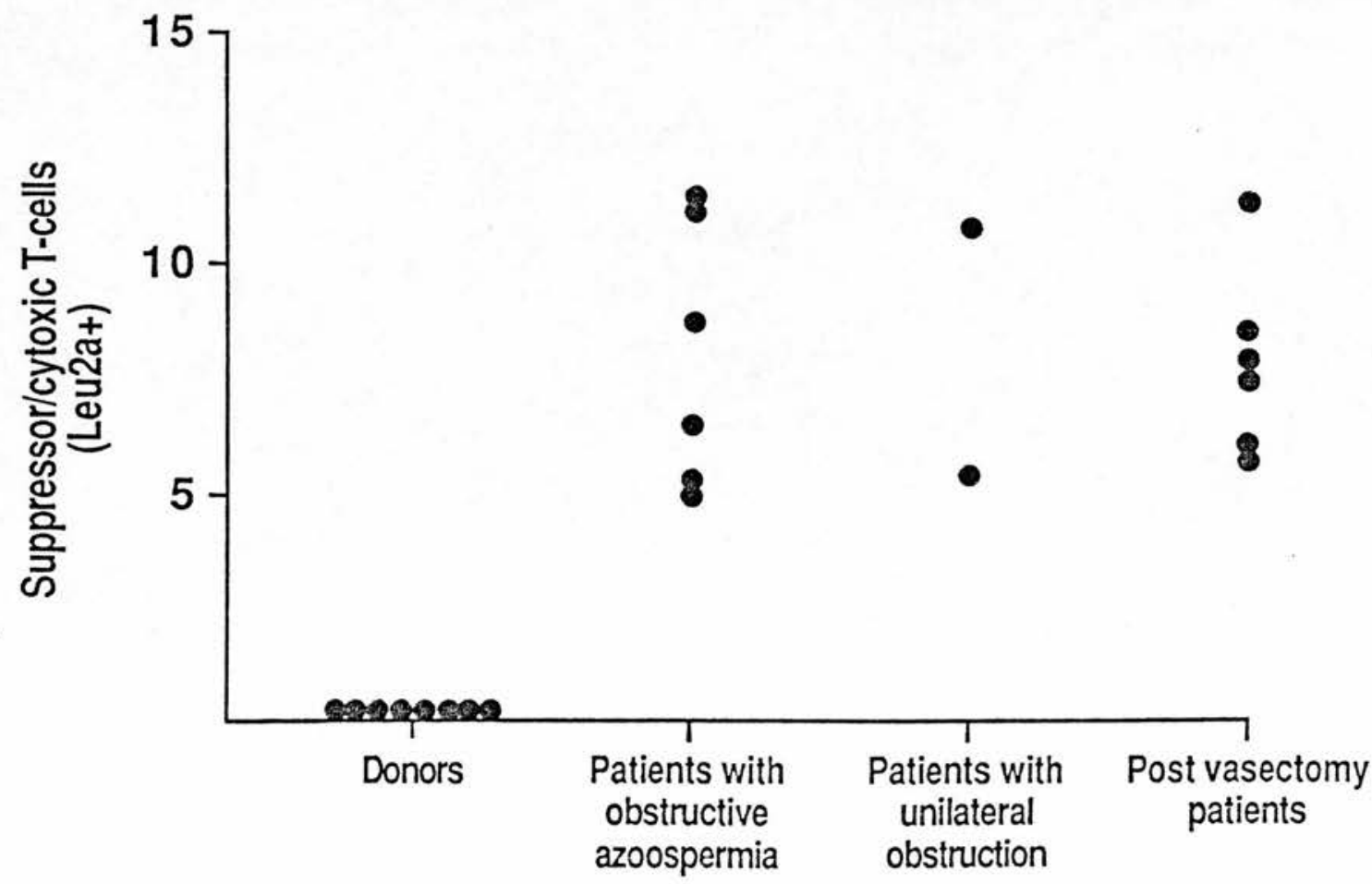




\section{IV.B. THE EPIDIDYMIS}

\section{IV.B.1 Normal Epididymal Tissues}

\section{IV.B.1.1 Histology}

The sections from the different epididymal regions showed no microscopical abnormalities on haematoxylin and eosin staining.

\section{IV.B.1.2 T-1ymphocytes}

Leu $4^{+}$cells (T-1ymphocytes) were demonstrable throughout the length of the epididymis within the actual epithelial lining, in the subepithelial connective tissue surrounding the epididymal ducts (lamina propria), and in the interstitial connective tissues. The greatest proportion of these T-cells were identifiable within the epithelium where they were situated alongside the epithelial cells and often in close proximity to the basement membrane. No positively stained cells were seen within the lumina of the epididymis (Figures IV.6a, b and c).

Within the epithelium, T-lymphocytes were estimated to represent $11.6 \%$ of the entire epithelial cell population. Up to $87.7 \%$ of these T-cells exhibited the suppressor/cytotoxic phenotype, Leu $2 \mathrm{a}^{+}$(Table IV.6). Counts of the T-1ymphocytes (Leu $4^{+}$cells) and $\mathrm{T}$-1ymphocyte subsets (Leu $2 \mathrm{a}^{+}$and Leu $3 \mathrm{a}^{+}$cells) within the epithelium showed no significant differences between the head, body and tail of the epididymis. 


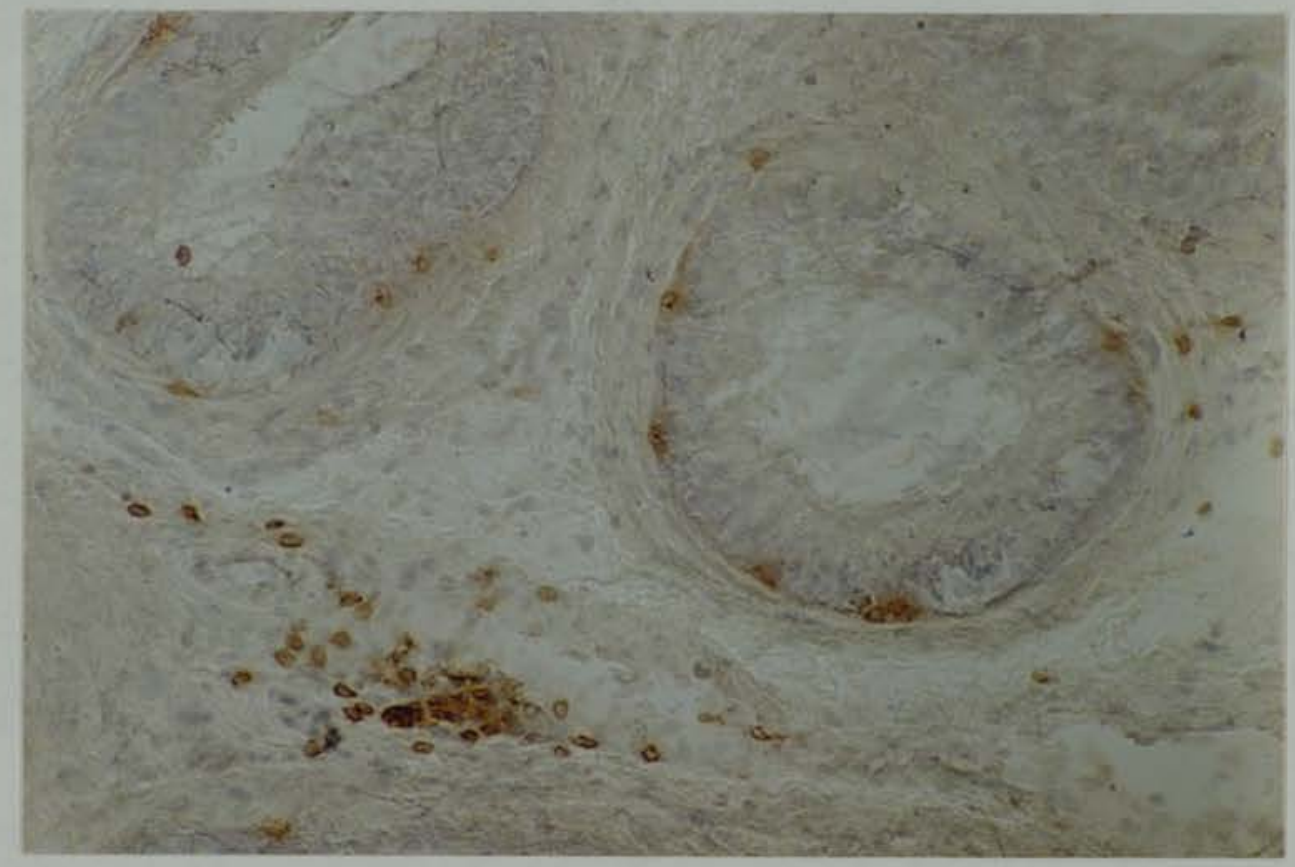

Figure IV.6a: Immune histological staining of a cryostat section of the body of the epididymis for T-1ymphocytes using the monoclonal antibody Leu 4 .

Note that these Leu $4^{+}$cells are located in the epithelium, lamina propria and in the interstitial connective tissue ( $x 160$ ). 


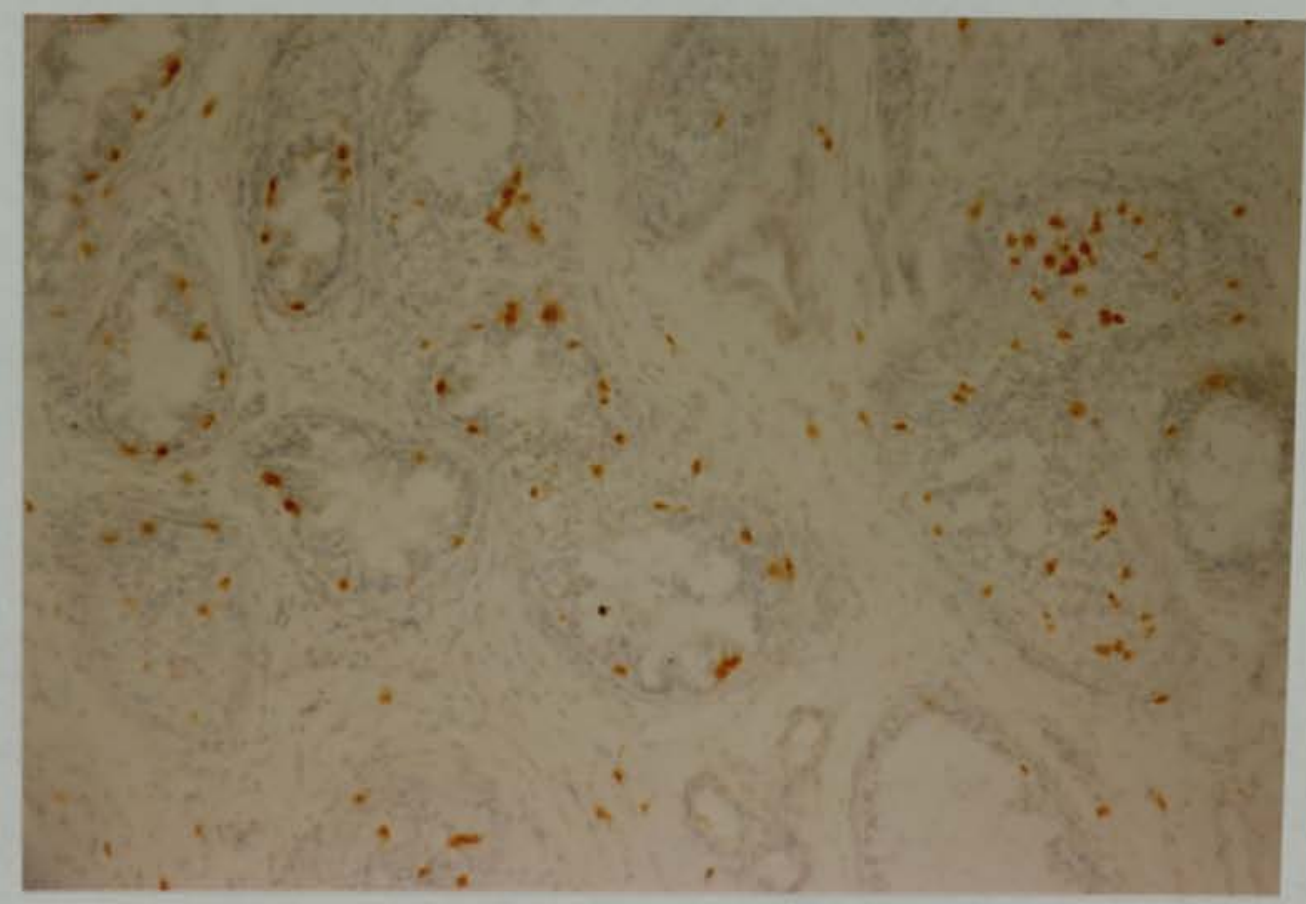

Figure IV.6b: Suppressor/cytotoxic T-cells (Leu $2 \mathrm{a}^{+}$) within the epithelium of the head of the epididymis.

Note the predominant location of these cells in the basal layer of the lining epithelium. (Cryostat section, immunoperoxidase, x 160). 


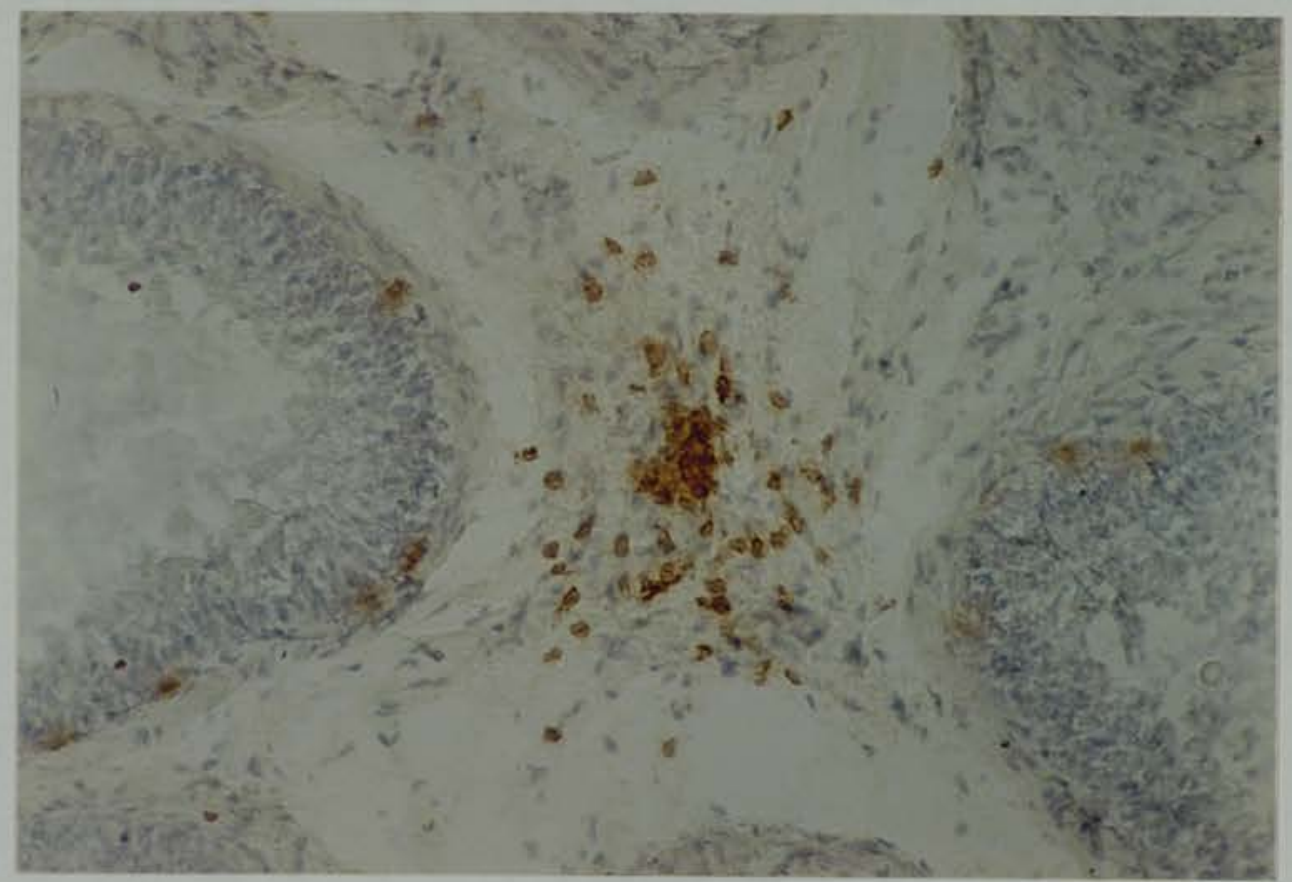

Figure IV.6c: Helper/inducer T-cells (Leu $3 a^{+}$) in the tail of the epididymis.

Note that these Leu $3 \mathrm{a}^{+}$cells are located mainly in the interstitial tissues. (Cryostat section, immunoperoxidase, x 160). 
TABLE IV.6:

MEAN (S.D.) LYMPHOCYTE COUNTS PER 100 EPITHELIAL CELLS WITHIN THE EPITHELIUM OF EPIDIDYMIS FROM 8 DONORS AND 6 PATIENTS

$\begin{array}{lll}\text { Leu } 2 \mathrm{a}^{+} \text {cells Leu } 3 \mathrm{a}^{+} \text {cells } \\ \text { Leu } 4^{+} \text {cells (T suppressor/ (T helper/ } & \text { Leu 2a/ } \\ \text { (All T-cells) cytotoxic) } & \text { inducer) } & \text { Leu 3a }\end{array}$

\begin{tabular}{|c|c|c|c|c|}
\hline$\overline{\text { Head }}$ ) & $12.3(3.3)$ & $10.1(2.7)$ & $2.4(0.7)$ & $4.45(1.17)$ \\
\hline Body ) & $10.7(2.6)$ & $8.2(2.0)$ & $2.4(1.3)$ & $4.10(1.70)$ \\
\hline Tail) & $11.8(1.2)$ & $9.1(1.2)$ & $2.7(1.1)$ & $3.95(2.03)$ \\
\hline$\frac{\text { Patients }}{\text { (epididymal }}$ & & & & \\
\hline biopsies) & $8.0(1.5)$ & $5.0(2.4)$ & $2.5(0.7)$ & $2.20(1.51)$ \\
\hline
\end{tabular}

TABLE IV.7:

MEAN (S.D.) LYMPHOCYTE COUNTS PER CROSS SECTION OF EPIDIDYMAL DUCT IN LAMINA PROPRIA OF EPIDIDYMIS FROM 8 DONORS AND 6 PATIENTS

\begin{tabular}{|c|c|c|}
\hline $\begin{array}{l}4^{+} \text {cells } \\
11 \text { T-cells) }\end{array}$ & $\begin{array}{c}\text { Leu } 2 \mathrm{a}^{+} \text {cells } \\
\text { ( } \mathrm{T} \text { suppressor } \\
\text { cytotoxic) }\end{array}$ & $\begin{array}{c}\text { Leu } 3 \mathrm{a}^{+} \text {cells } \\
\text { (T helper/ } \\
\text { inducer) }\end{array}$ \\
\hline
\end{tabular}

\begin{tabular}{ccccc} 
Donors & & & & \\
\hline Head ) & $1.7(0.3)$ & $1.3(0.3)$ & $0.9(0.2)$ & $1.50(0.30)$ \\
$\begin{array}{c}\text { Body ) } \\
\text { Tail ) }\end{array}$ & $2.3(0.6)$ & $1.6(0.4)$ & $1.1(0.4)$ & $1.68(0.84)$ \\
$\begin{array}{l}\text { Patients } \\
\begin{array}{l}\text { (epididyma1 } \\
\text { biopsies) }\end{array}\end{array}$ & $6.6(2.9)$ & $2.6(0.6)$ & $1.5(0.4)$ & $1.74(0.33)$ \\
\hline
\end{tabular}

TABLE IV.8:

MEAN (S.D.) LYMPHOCYTE COUNTS PER HPF ( $x$ 160) IN INTERSTITIAL TISSUE OF EPIDIDYMIS FROM 8 DONORS AND 6 PATIENTS

$\begin{array}{lcc}\text { Leu } 2 \mathrm{a}^{+} \text {cells } & \text { Leu } 3 \mathrm{a}^{+} \text {cells } \\ \text { Leu } 4^{+} \text {cells } & \text { (T suppressor/ (T helper/ } & \text { Leu 2a/ } \\ \text { (A11 T-cells) cytotoxic) } & \text { inducer) } & \text { Leu 3a }\end{array}$

\begin{tabular}{ccccc} 
Donors & & & & \\
\hline Head ) & $8.9(6.2)$ & $3.4(1.6)$ & $7.7(5.6)$ & $0.52(0.17)$ \\
Body ) & $7.4(4.1)$ & $2.8(1.4)$ & $5.9(3.2)$ & $0.52(0.17)$ \\
$\begin{array}{c}\text { Tail } \\
\text { Patients }\end{array}$ & $8.5(2.6)$ & $3.0(1.0)$ & $6.3(2.9)$ & $0.56(0.29)$ \\
$\begin{array}{l}\text { (epididymal } \\
\text { biopsies) }\end{array}$ & $35.3(15.1)$ & $8.6(4.0)$ & $28.6(12.6)$ & $0.31(0.08)$ \\
\hline
\end{tabular}

(See Appendix B) 
Within the lamina propria the suppressor/cytotoxic T-cells, Leu $2 \mathrm{a}^{+}$, predominated at all levels of the epididymis (Table IV.7). Furthermore, the Leu $4^{+}$cells, all T-cells, and the Leu $2 \mathrm{a}^{+}$cells were present at significantly higher counts $(P<0.05)$ within the epididymal tail as compared with the body or the head.

There was a random distribution of $\mathrm{T}$-1ymphocytes within the interstitial connective tissues. They usually occurred singly but occasional small, perivascular and perilymphatic lymphoid aggregates were noted. The T-1ymphocytes at this site were predominantly of the helper/inducer phenotype, Leu $3 \mathrm{a}^{+}$cells (Table IV. 8 and Figure IV.6c). There were no significant differences in the T-cell counts between the head, body and tail.

None of the T-1ymphocytes showed a detectable reaction within the anti-interleukin-2 receptor monoclonal antibody, which detects activated T-cells.

\section{IV.B.1.3 B-1ymphocytes}

The B-1ymphocytes (Leu $14^{+}$cells) were rarely encountered in the epithelium of the epididymis. However, occasional Leu $14^{+}$ cells were seen in the lamina propria and in the interstitial connective tissues.

\section{IV.B.1.4 Macrophages}

Similar patterns of staining were obtained with the Leu M3, 3.9 and 24 monoclonal antibodies. Leu M3 ${ }^{+}, 3.9^{+}$and $24^{+}$cells were detected (like the T-lymphocytes) within the epithelium, within the 
lamina propria and within the interstitial connective tissues around the blood vessels and lymphatics (Figure IV.7).

\section{IV.B.1.5 Anti-HLA-DR reactive cells}

Along the length of the epididymis, the cell membranes of all types of the epithelial cells and vascular endothelia were stained with the anti-HLA-DR monoclonal antibody. Spermatozoa within the lumen of the epididymis were HLA-DR negative. Cells with a strong staining intensity were seen within the epithelium, lamina propria and the interstitial tissues. The labelling pattern of the anti-HLA-DR antibody (Figure IV.8) overlapped completely that of the antimacrophage (Leu M3, 3.9 and 24) antibodies. This suggests that the majority of these cells are macrophages expressing HLA-DR antigens. 

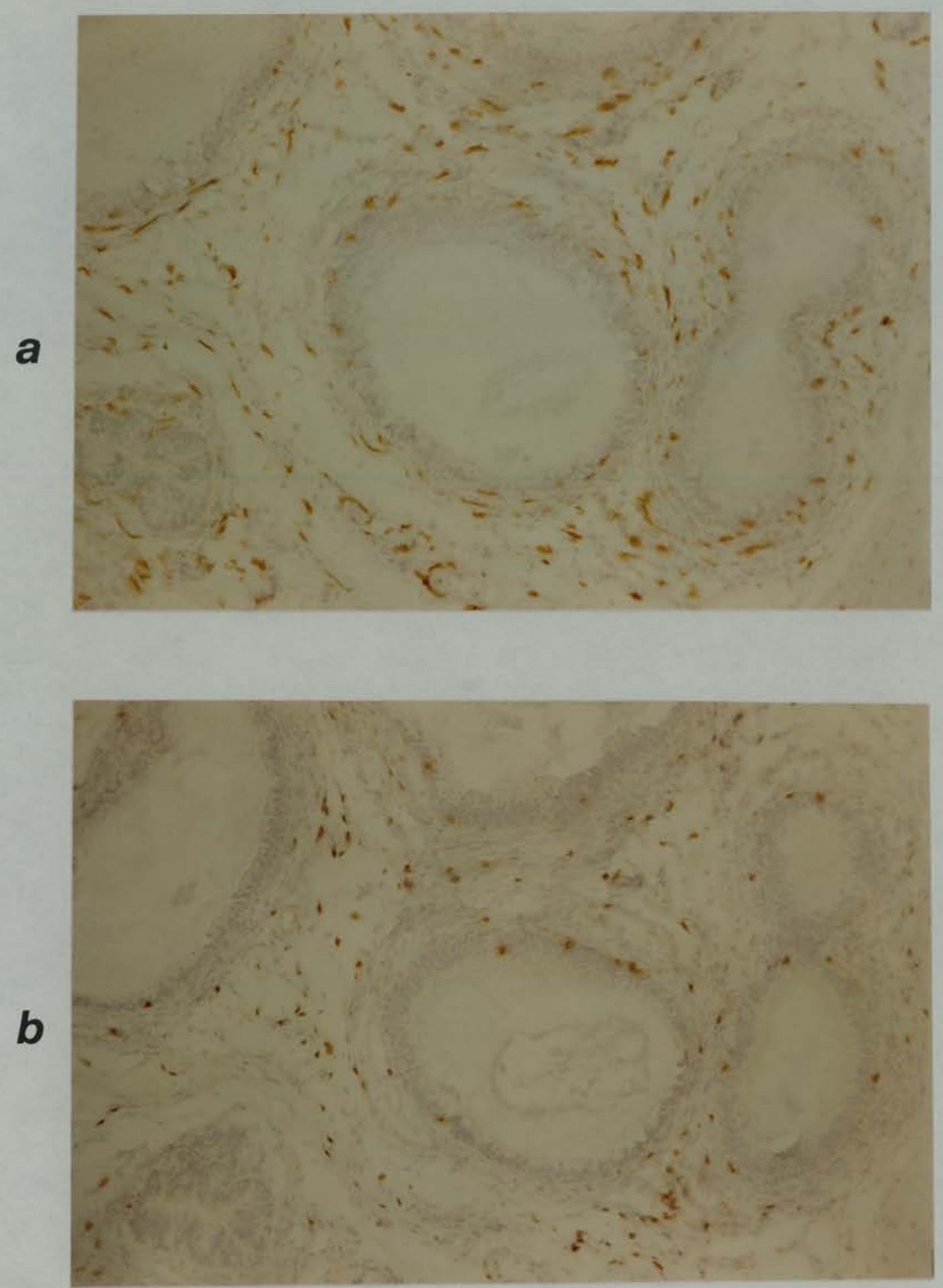

Figure IV.7: (a) Leu $\mathrm{M3}^{+}$cells (monocytes/macrophages) in normal epididymis. Note that these cells are located, like T-cells, within the epithelium, lamina propria and interstitial tissues. See Figure (b), the same field stained for T-1ymphocytes.

(Cryostat sections, immunoperoxidase, x 160) 


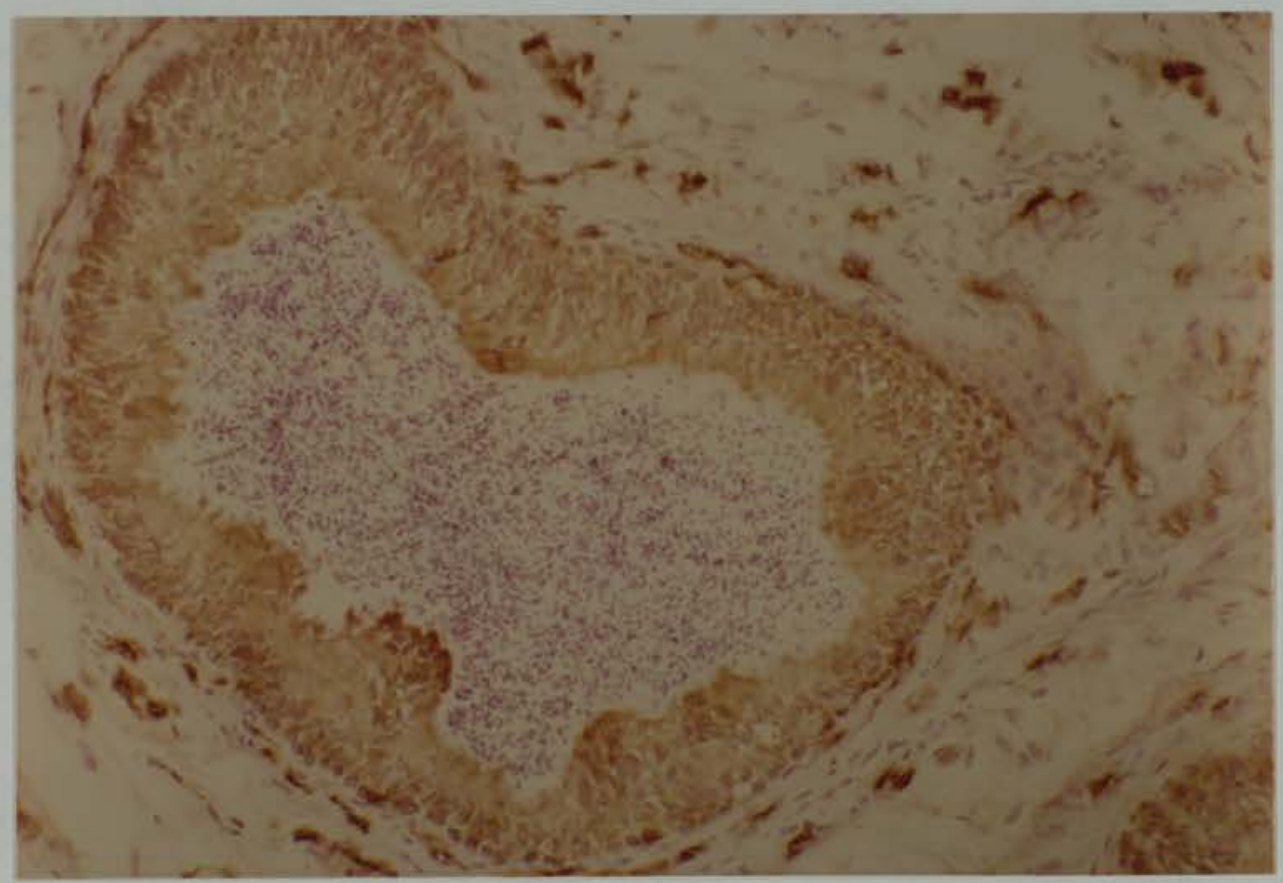

Figure IV.8: Immunoperoxidase staining of a cryostat section of the tail of the epididymis stained with the anti HLA-DR antibody.

Note the HLA-DR ${ }^{+}$cells in the lamina propria and the interstitial tissue, the expression of HLA-DR antigens on the lining epithelium and the lack of this expression on the luminal spermatozoa. 


\section{IV.B.2 Epididymal Biopsies from Patients with Testicular}

\section{Obstruction}

\section{IV.B.2.1 Histology}

Haematoxylin and eosin staining of the epididymal biopsies showed dilated epididymal tubules, increased stromal fibrosis and infiltration with mononuclear cells. No other abnormalities were seen.

\section{IV.B.2.2 Immunohistology}

Within the epithelium, the numbers of the suppressor/ cytotoxic cells, Leu $2 \mathrm{a}^{+}$, were diminished. The counts of Leu $4^{+}$ cells and Leu $2 \mathrm{a}^{+}$cells were significantly lower in these patients as compared with biopsies from normal donors $(P<0.05)$, but there were no significant differences in the Leu $3 a$ positive cell counts (Table IV.6 and Figure IV.9).

In contrast, there was a marked increase in the T-helper/ inducer cells (Leu $3 \mathrm{a}^{+}$), within the lamina propria and in the interstitial tissue. The Leu $4^{+}$and Leu $3 \mathrm{a}^{+}$cell counts were significantly higher and the Leu $2 \mathrm{a}^{+} /$Leu $3 \mathrm{a}^{+}$ratios were significantly lower in patients as compared with the donors (all at P $<0.01$ ), (Tables IV.7 and 8; Figures IV.10, 11 and 12).

The three patients with obstructive azoospermia showed higher counts of Leu $2 \mathrm{a}^{+}$cells (the T-suppressor/cytotoxic cells) within the epithelium and the lamina propria as compared with both the patients with unilateral obstruction and those undergoing 
vasectomy reversal. The latter group showed higher counts of Leu $3 \mathrm{a}^{+}$cells (T-helper/inducer cells) within the epithelium and the lamina propria.

The anti-interleukin 2-receptors antibody, which detects activated T-cells, stained a substantial number of cells in the lamina propria and in the interstitial tissue. This was noted in the tissues from both the patients with unilateral obstruction and with vasectomy reversal. Increased infiltration by macrophages was also demonstrated in these patients (Figure IV.13), and antisperm antibodies were detected in high titres in their serum and seminal plasma, while no sperm antibodies were detected in the patients with azoospermia (see Tables IV.3, 4 and 5). 


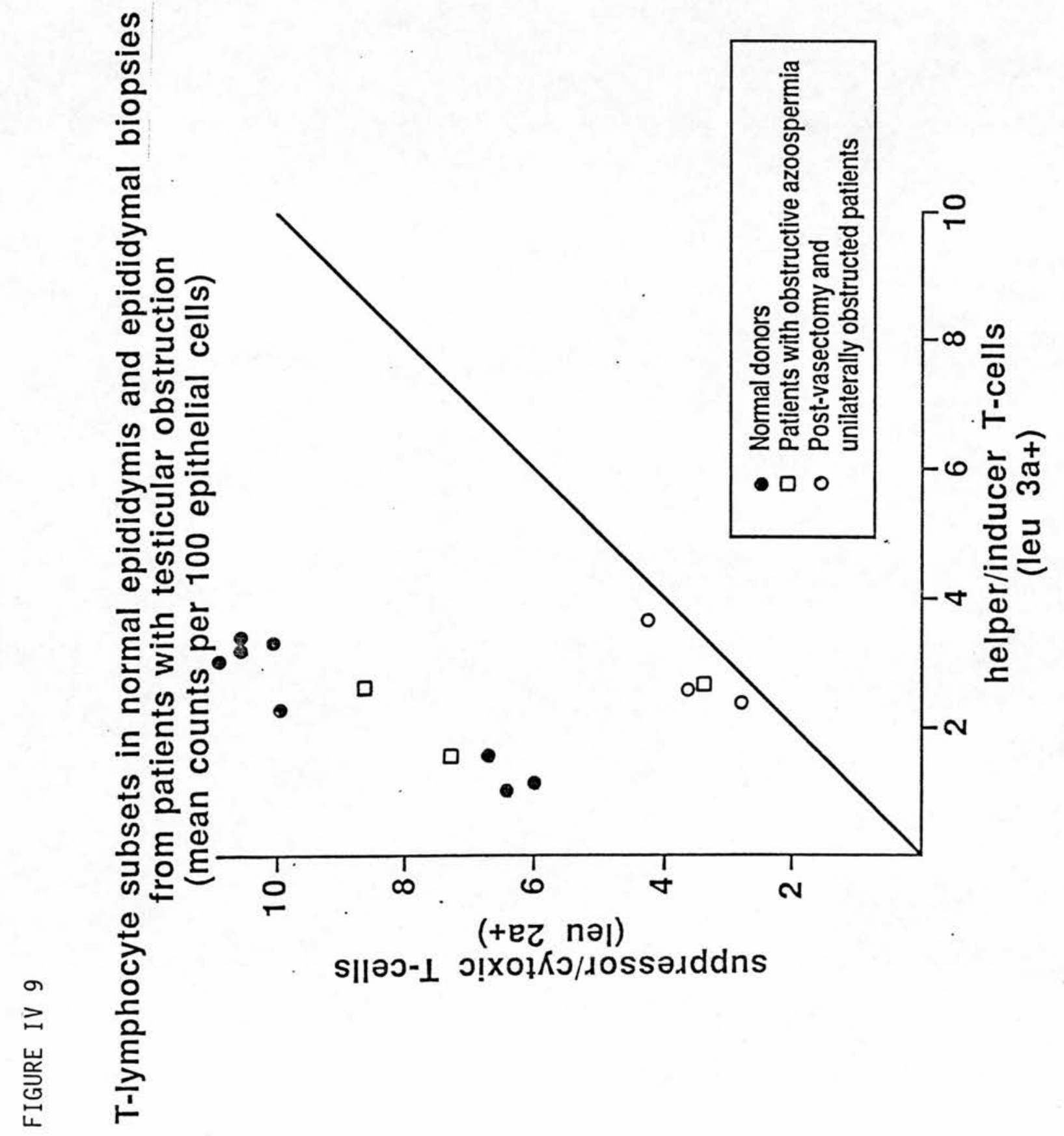




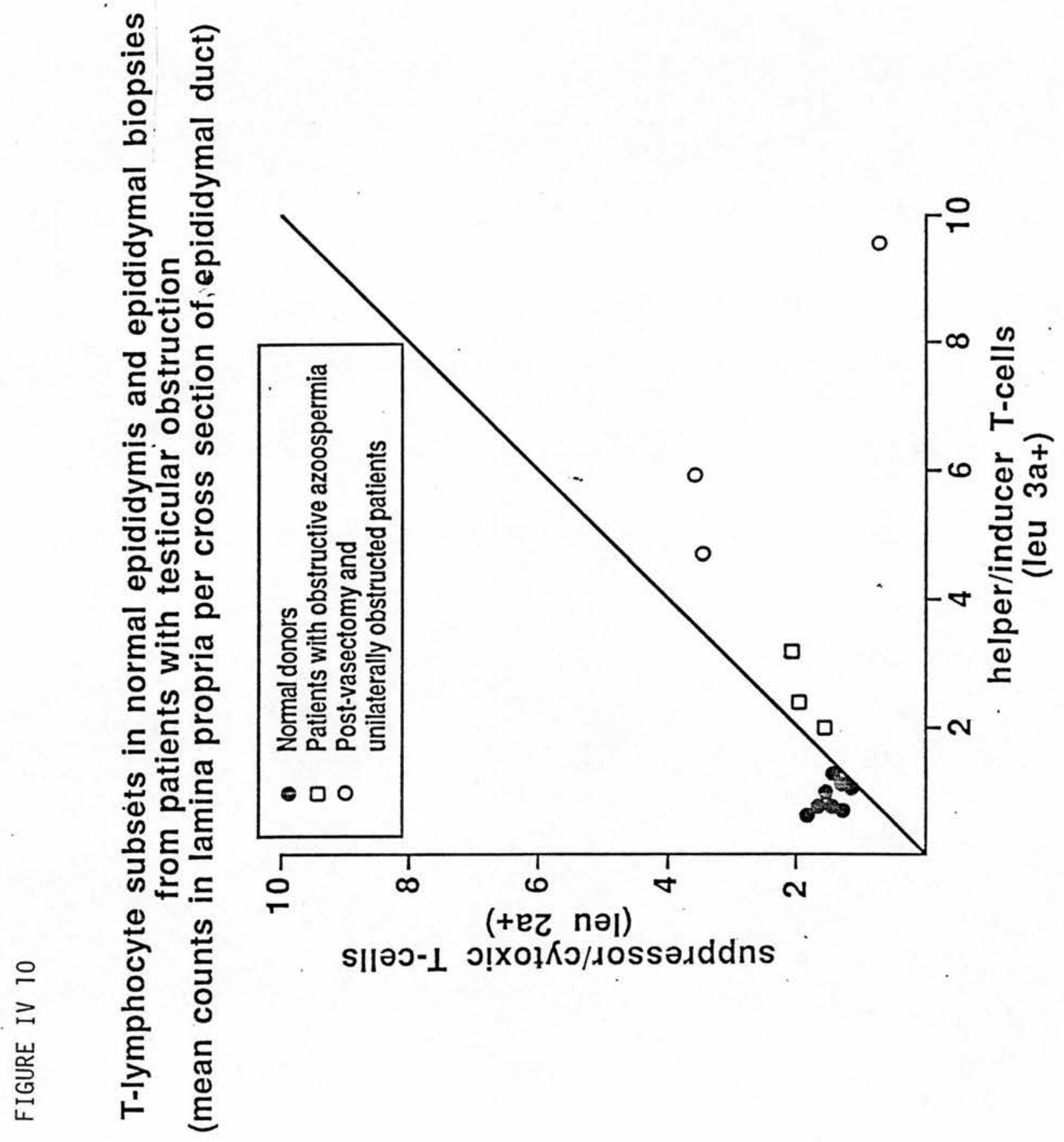




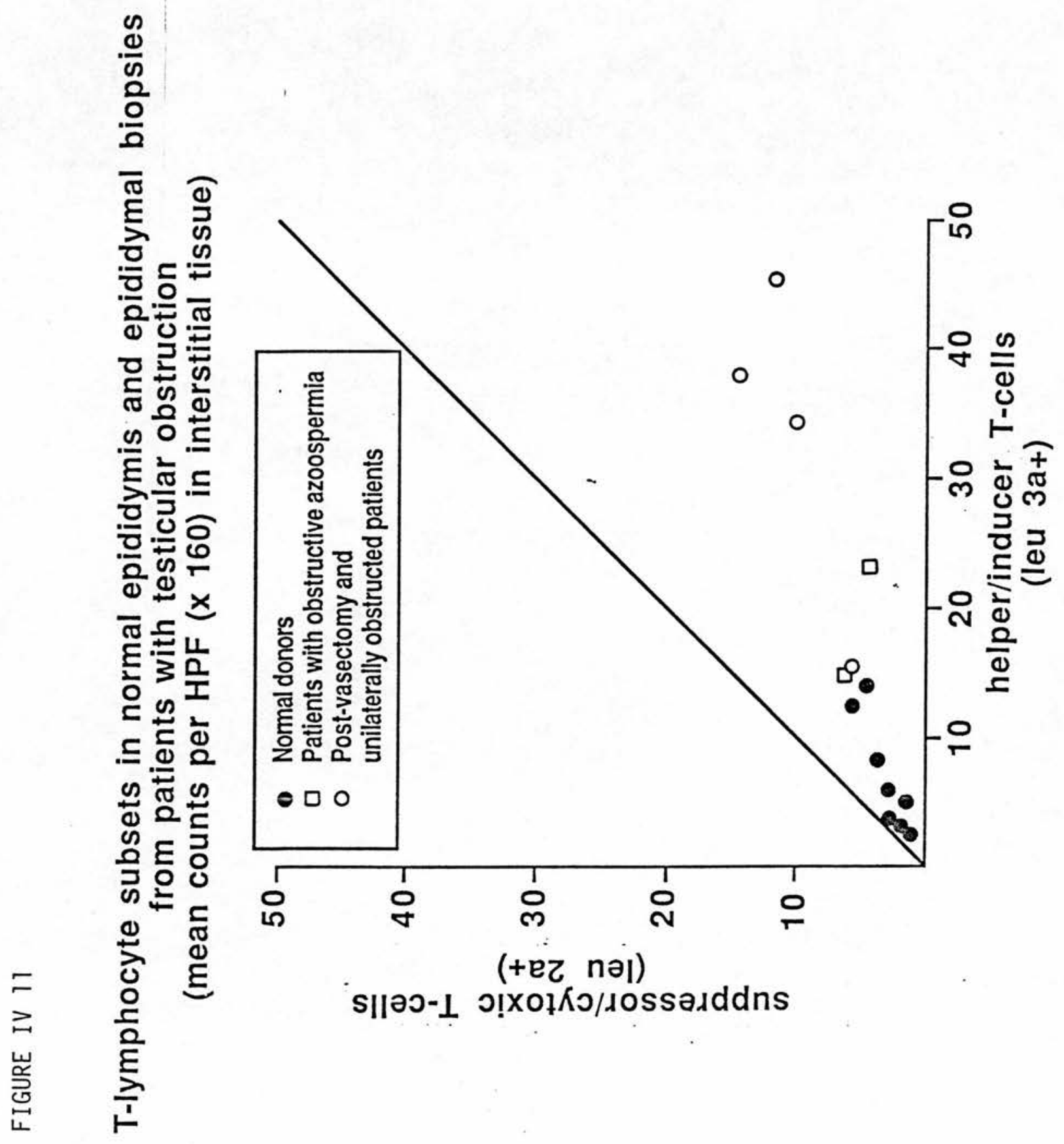



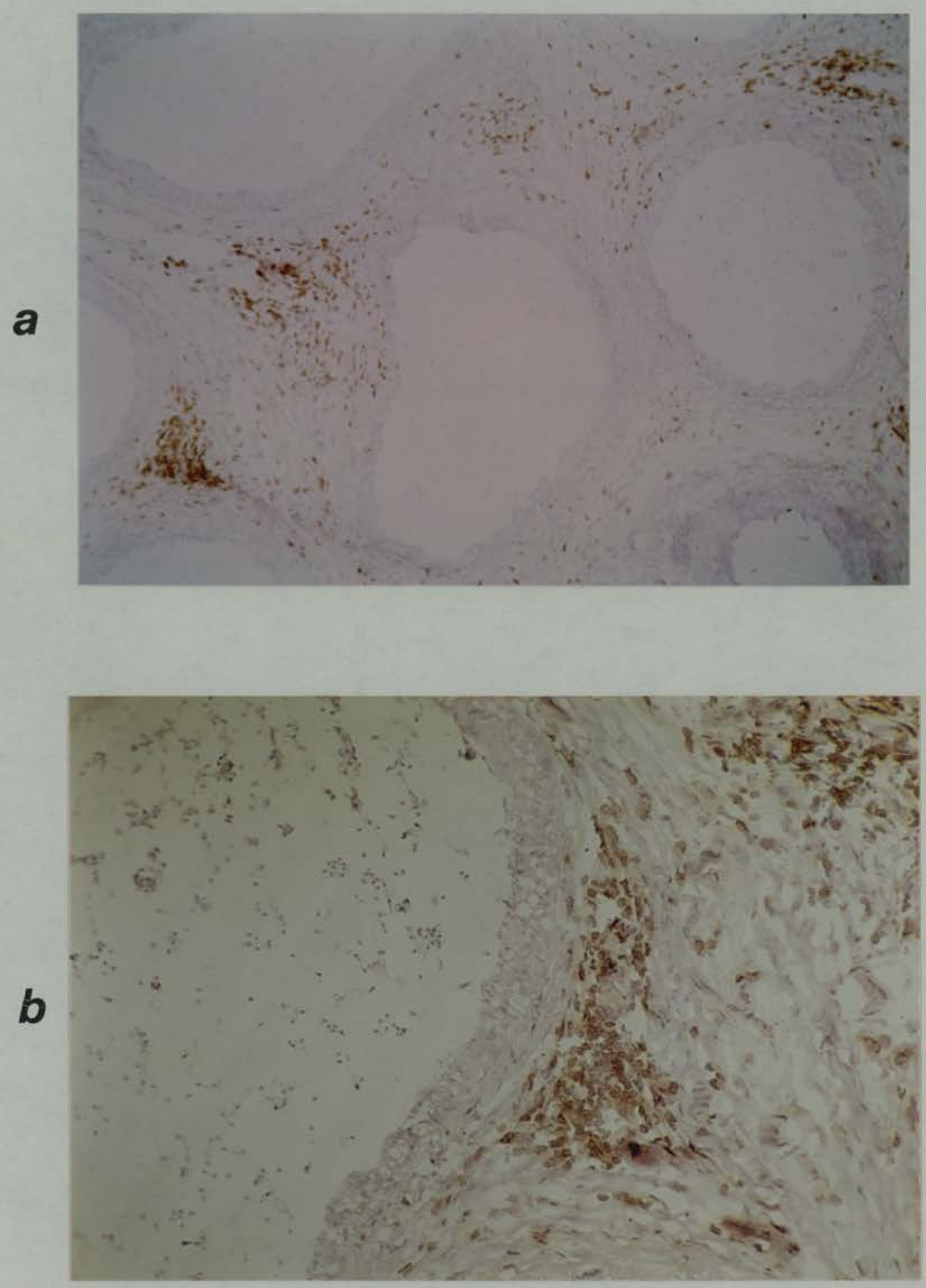

Figure IV.12: Immunoperoxidase staining of cryostat sections of epididymal biopsy from a vasectomy reversal patient stained with (a) Leu 4 antibody for all T-cells, and (b) Leu 3a antibody for helper/inducer cells.

Note loss of the T-cells from the epithelium and increased infiltration of the lamina propria and interstitium with Leu $3 \mathrm{a}^{+}$ cells. (x 160). 

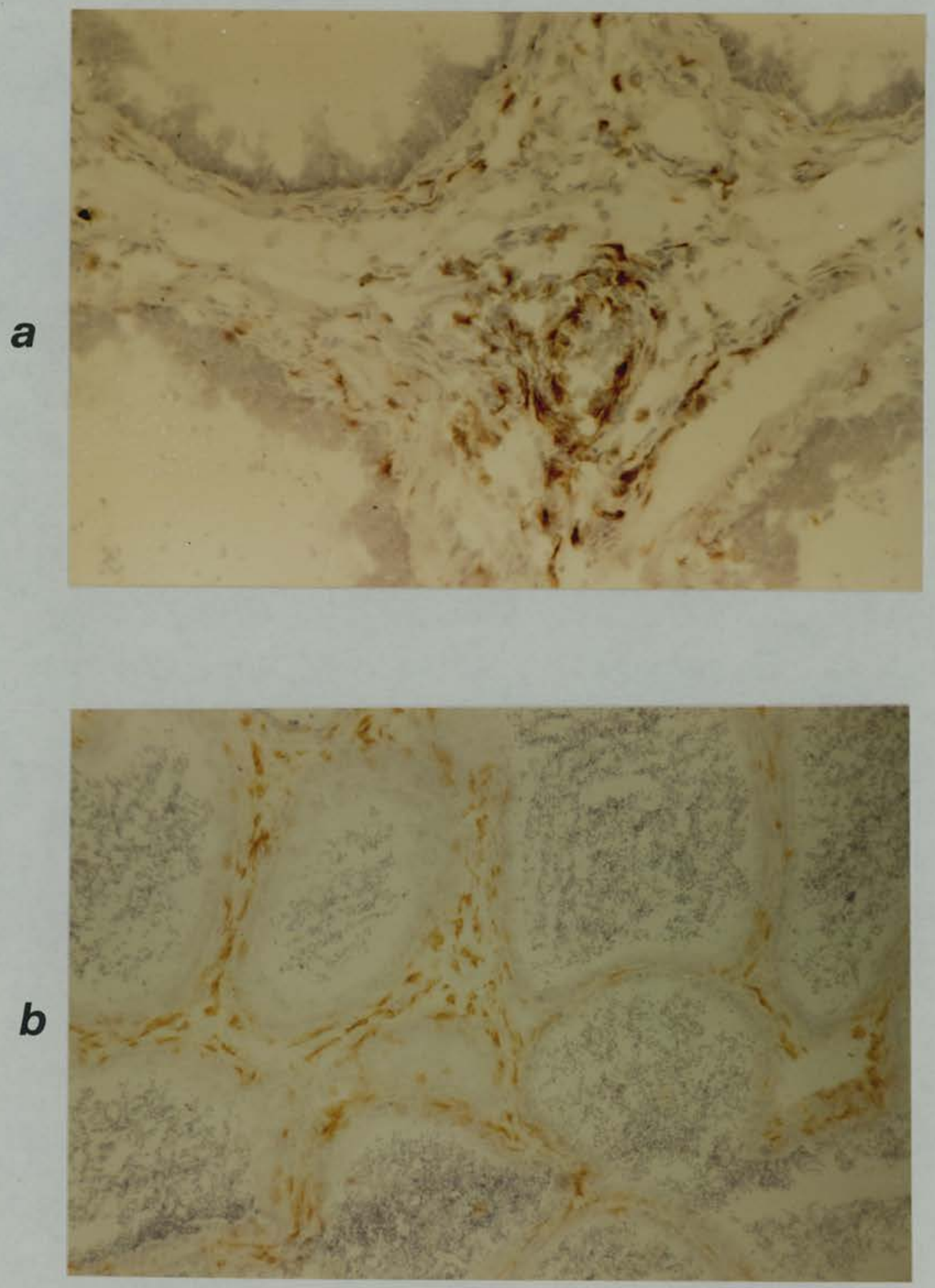

Figure IV.13: Leu $\mathrm{M3}^{+}$cells (macrophages) in epididymal biopsies from: (a) a vasectomy reversal patient and (b) a unilaterally obstructed patient.

(Cryostat sections, immunoperoxidase, x 160) 
IV.C THE VAS DEFERENS

\section{IV.C.1 Lymphocytes}

\section{IV.C.1.1 The vasal part}

In the scrotal portion of the vas deferens $(n=8)$, a T-lymphocyte population (Leu $4^{+}$) was demonstrated in between the epithelial cells and within the lamina propria (Figure IV.14). Within the epithelium these T-1ymphocytes were located mainly in the basal layer of the epithelium and constituted approximately $25 \%$ of the epithelial cell population. Up to $63 \%$ of this T-cell population were suppressor/cytotoxic T-cells (Leu $2 \mathrm{a}^{+}$) (Table IV.9). The suppressor/cytotoxic T-cells predominated also in the lamina propria. The anti-Leu 3 a antibody, which defines the helper/inducer T-cells, stained a minority of the T-cell population. No B-lymphocytes were detected.

Table IV. 9 and Figures IV.15 and 16 show the reactivity of the anti-T-1ymphocyte antibodies from two different sources (B.D. and C.C.) with the vas deferens from cadaver and live donors. There were no significant differences between the T-1ymphocyte counts as measured by the B.D. antibodies and those measured by the C.C. reagents, nor in the Leu $2 \mathrm{a} /$ Leu $3 \mathrm{a}(\mathrm{T} 8 / \mathrm{T} 4)$ ratios. Live donors and cadavers did not differ significantly in any of the T-1ymphocyte counts. 

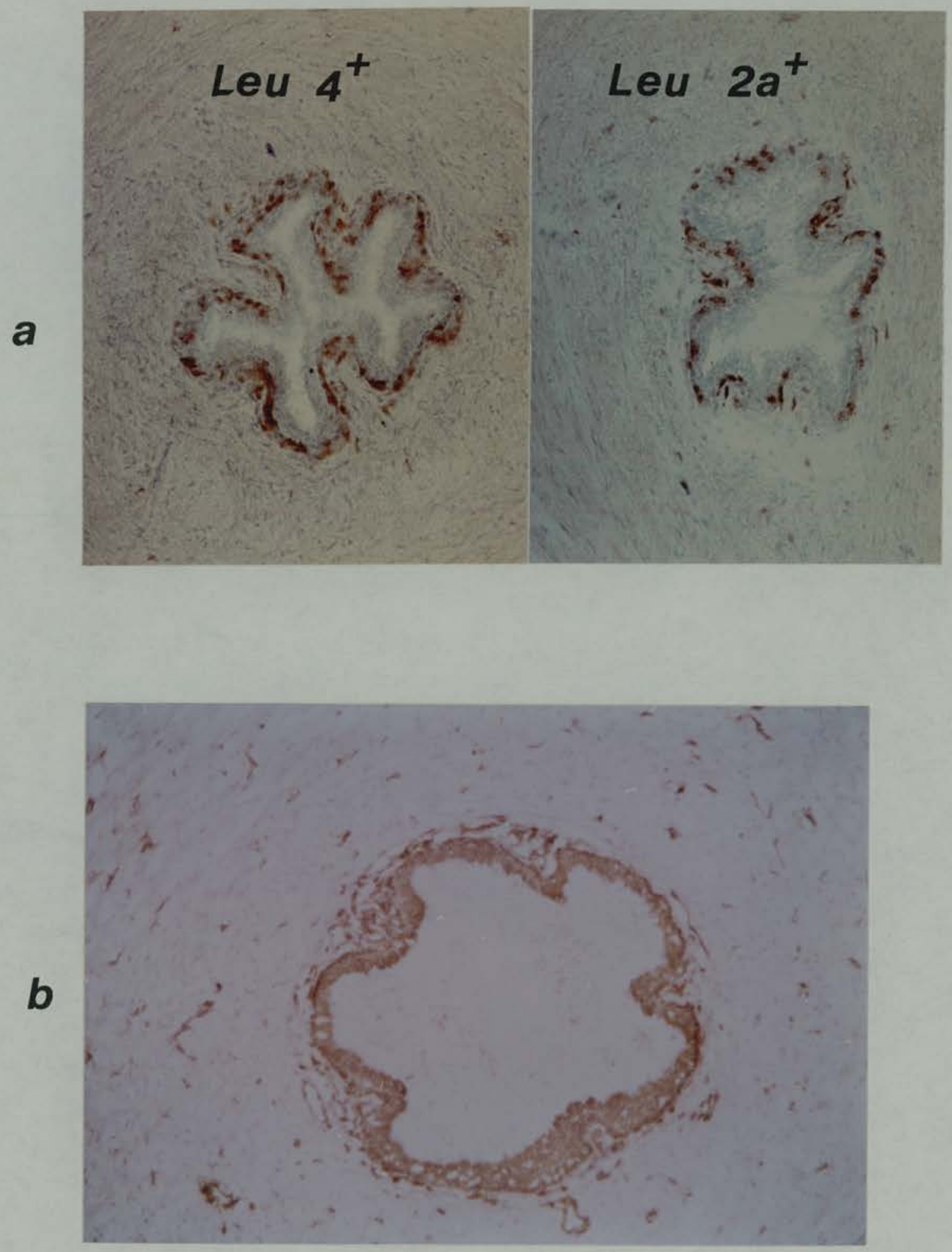

Figure IV.14: Immunoperoxidase staining of cryostat sections of vas deferens stained for:

(a) T-1ymphocytes. Note the location of these T-cells in the basal layer of the epithelium;

(b) HLA-DR antigens. Note the expression of HLA-DR antigens on the epithelium. 
TABLE IV.9: T-LYMPHOCYTE SUBSETS IN THE VAS DEFERENS

\begin{tabular}{|c|c|c|c|c|}
\hline Donor & $\begin{array}{l}\text { Leu } 4^{+} \text {cells }(\mathrm{T} \\
\text { (A11 T-cells) }\end{array}$ & $\begin{array}{l}\text { eu } 2 \mathrm{a}^{+} \text {cells } \mathrm{L} \\
\text { suppressor/ } \\
\text { cytotoxic) }\end{array}$ & $\begin{array}{l}3 \mathrm{a}^{+} \text {cells } \\
\text { helper/ } \\
\text { inducer) }\end{array}$ & $\begin{array}{l}\text { Leu } 2 a / \\
\text { Leu } 3 a\end{array}$ \\
\hline \multicolumn{5}{|c|}{ A. EPITHELIUM (mean counts \pm S.D. per 100 epithelial cells) } \\
\hline \multicolumn{5}{|l|}{ Cadavers } \\
\hline$\overline{\mathrm{D} 1}$ & 23.92 & 15.33 & 9.07 & 1.69 \\
\hline D2 & 25.64 & 18.31 & 9.77 & 1.87 \\
\hline D3 & 32.83 & 22.78 & 9.48 & 2.40 \\
\hline \multicolumn{5}{|l|}{ Vasectomy } \\
\hline \multirow{5}{*}{$\begin{array}{r}\text { Patients } \\
349317 \\
386367 \\
385040\end{array}$} & & & & \\
\hline & 21.95 & 14.14 & 6.64 & 2.12 \\
\hline & 20.31 & 12.24 & 10.02 & 1.22 \\
\hline & 25.55 & 12.12 & 8.566 & 1.41 \\
\hline & T3 & T8 & $\mathrm{T} 4$ & $\mathrm{~T} 8 / 4$ \\
\hline 385040 & 27.82 & 12.36 & 7.63 & 1.61 \\
\hline 368820 & 24.0 & 13.46 & 9.31 & 1.44 \\
\hline 386573 & 21.64 & 15.61 & 7.88 & 1.98 \\
\hline A11 donors & $24.84 \pm 3.78$ & $15.15 \pm 3.49$ & $8.70 \pm 1.12$ & $1.74 \pm 0.37$ \\
\hline
\end{tabular}

Leu $2 a^{+}$cells Leu $3 a^{+}$cells

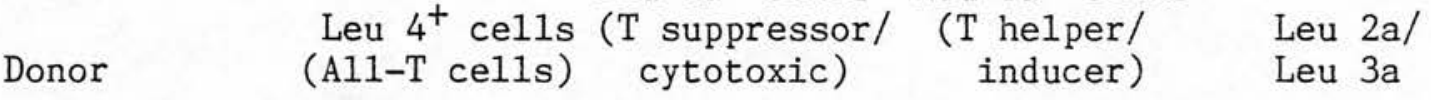

B. LAMINA PROPRIA (mean counts \pm S.D. per cross section of the vas)

Cadavers

\begin{tabular}{ccccc} 
D1 & $14.4 \pm 1.5$ & $8.4 \pm 2.1$ & $5.7 \pm 1.9$ & 1.47 \\
D2 & $15.2 \pm 2.0$ & $7.2 \pm 1.8$ & $4.0 \pm 1.8$ & 1.8 \\
D3 & $11.7 \pm 1.7$ & $4.3 \pm 2.2$ & $2.4 \pm 1.2$ & 1.8 \\
Vasectomy & & & & \\
\hline Patients & & & & \\
\hline 349317 & $9.2 \pm 1.4$ & $6.0 \pm 1.1$ & $3.2 \pm 2.2$ & 1.9 \\
386367 & $9.3 \pm 2.3$ & $5.1 \pm 1.2$ & $4.3 \pm 1.2$ & 1.2 \\
385040 & $9.1 \pm 2.5$ & $5.2 \pm 2.3$ & $2.4 \pm 1.0$ & 2.2 \\
& & & & $\mathrm{~T} 4$ \\
385040 & $10.9 \pm 2.0$ & $5.4 \pm 1.3$ & $3.5 \pm 0.8$ & 1.5 \\
368820 & $8.8 \pm 1.9$ & $6.0 \pm 1.9$ & $4.6 \pm 1.7$ & 1.3 \\
386573 & $17.1 \pm 1.7$ & $12.3 \pm 1.5$ & $9.0 \pm 1.6$ & 1.4 \\
& & & & \\
A11 donors & $11.7 \pm 3.06$ & $6.65 \pm 2.44$ & $4.34 \pm 2.03$ & $1.6 \pm 0.32$ \\
\hline
\end{tabular}




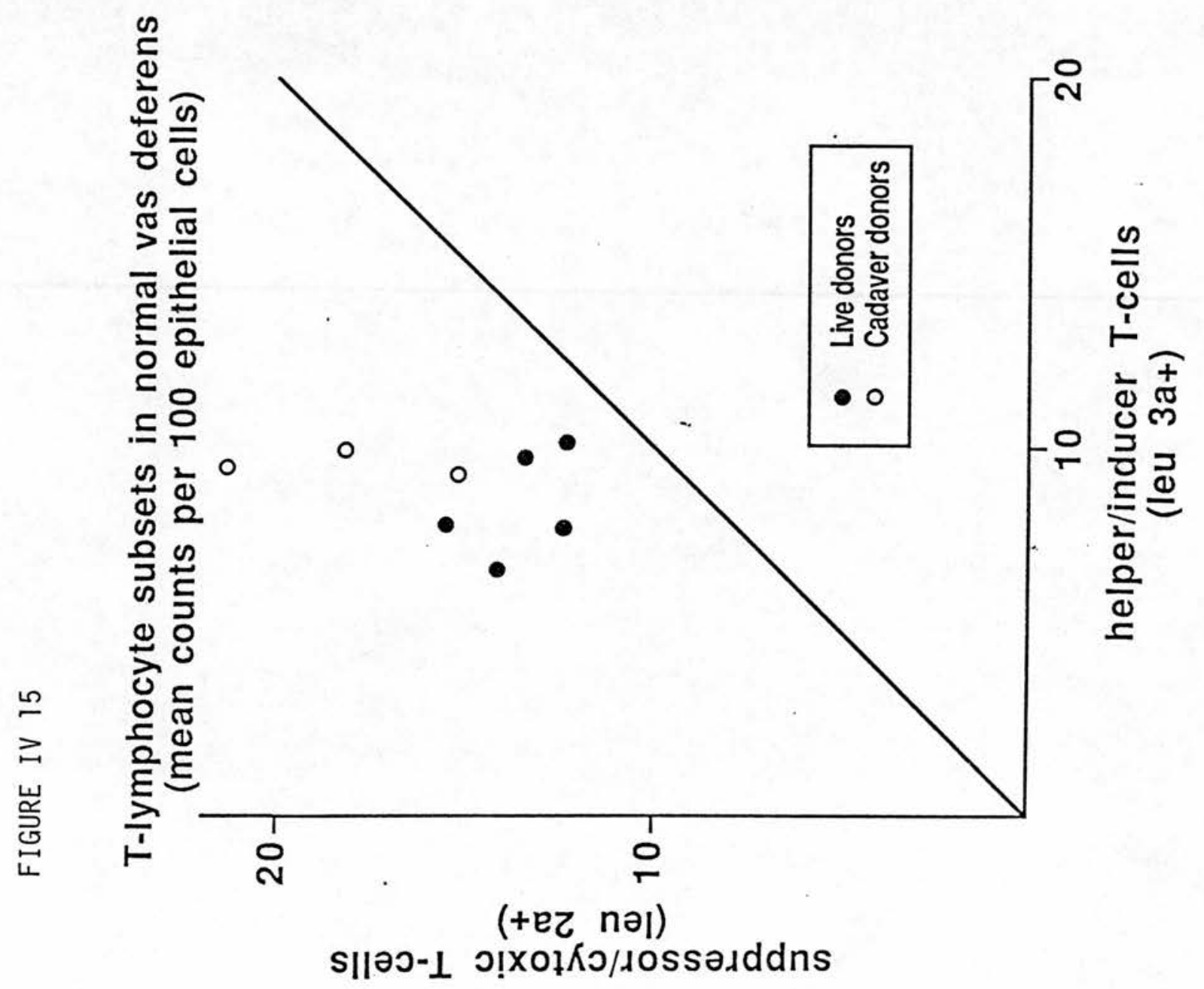




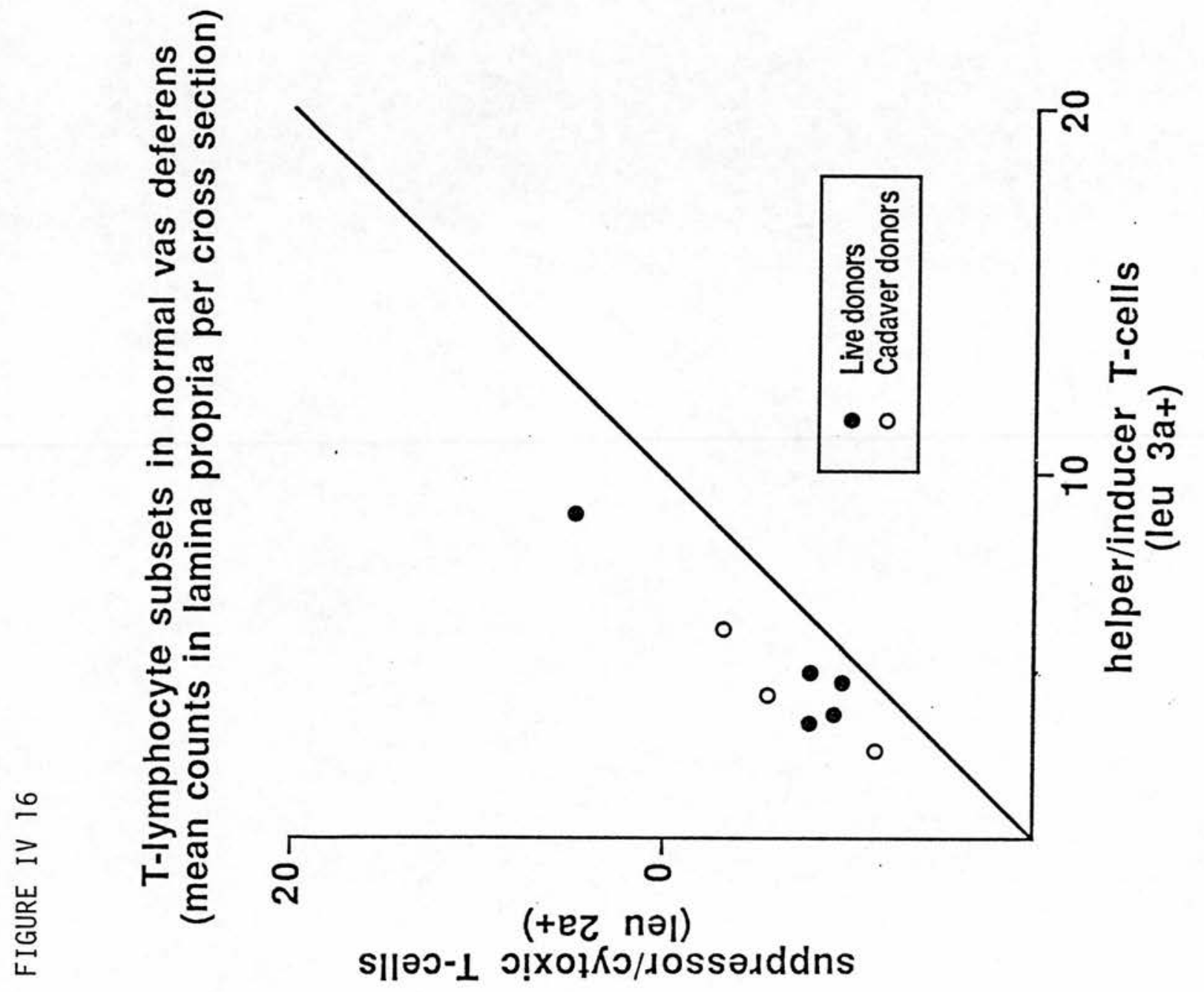




\section{IV.C.1.2 The ampulla of the vas}

T-1ymphocytes were also demonstrated within the convoluted epithelial lining and within the lamina propria of the ampulla of the vas. Within the epithelium they were found in the range 3.5-7.3 T-1ymphocytes/100 epithelial cells. They were mainly of the suppressor/cytotoxic phenotype (Leu $2 \mathrm{a}^{+}$). Table IV.10 shows the reactivity of the antilymphocyte antibodies within the epithelium and within the lamina propria of the ampulla of the vas. The T-1ymphocyte counts within the ampulla were significantly lower than those within the vasal part $(P<0.01)$.

\section{IV.C.2 Macrophages}

Cells reacting with the antimacrophage antibodies, Leu M3, 3.9 and 24 , were detected within the epithelium and within the lamina propria of both the vasal part and the ampulla of the vas deferens. They were few in numbers but appeared more numerous in the vasal (scrotal) part.

\section{IV.C.3 The Anti-HLA-DR Reactive Cells}

The lining epithelium of both the vasal part and the ampulla of the vas was completely stained with the anti-HLA-DR antibody (Figure IV.14). The staining was somewhat weaker in sections of the ampulla. HLA-DR positive cells were also seen in the lamina propria. 
TABLE IV.10:

T-LYMPHOCYTE SUBSETS IN THE AMPULLA OF THE VAS DEFERENS

\begin{tabular}{|c|c|c|c|}
\hline Donor & $\begin{array}{l}\text { Leu } 2 \mathrm{a}^{+} \text {cells } \\
\text { Leu } 4^{+} \text {cells ( } \mathrm{T} \text { suppressor/ } \\
\text { (A11 T-cells) cytotoxic) }\end{array}$ & $\begin{array}{l}\text { Leu } 3 \mathrm{a}^{+} \text {cells } \\
\text { ( } \mathrm{T} \text { helper/ } \\
\text { inducer) }\end{array}$ & $\begin{array}{l}\text { Leu } 2 a / \\
\text { Leu } 3 a\end{array}$ \\
\hline
\end{tabular}

$\underline{\text { EPITHELIUM }}$ (mean counts \pm S.D. per 100 epithelial cells)

$\begin{array}{lllll}\text { D1 } & 7.13 & 5.06 & 0.97 & 5.21 \\ \text { D2 } & 3.52 & 3.46 & 1.19 & 2.90 \\ \text { D3 } & 7.28 & 5.04 & 1.72 & 2.93 \\ \text { D4 } & 5.43 & 3.64 & 1.23 & 2.95\end{array}$

$\begin{array}{lllll}\text { All donors } & 5.84 \pm 1.76 & 4.3 \pm 0.87 & 1.27 \pm 0.31 & 3.49 \pm 1.14\end{array}$

CONNECTIVE TISSUES OF THE LAMINA PROPRIA AND INTERSTITIUM (mean counts \pm S.D. per $0.8 \mathrm{~mm}^{2}$ tissue $\mathrm{x} \mathrm{160)}$

$\begin{array}{lllll}\text { D1 } & 5.0 \pm 2.0 & 2.0 \pm 0.8 & 4.0 \pm 1.8 & 0.5 \\ \text { D2 } & 3.2 \pm 1.6 & 1.6 \pm 0.7 & 2.3 \pm 0.7 & 0.69 \\ \text { D3 } & 7.1 \pm 1.7 & 1.8 \pm 0.8 & 5.8 \pm 1.2 & 0.31 \\ \text { D4 } & 6.0 \pm 1.8 & 2.3 \pm 1.8 & 2.8 \pm 1.6 & 0.82\end{array}$

$\begin{array}{lllll}\text { Al1 donors } & 5.32 \pm 1.65 & 1.92 \pm 0.29 & 3.72 \pm 1.55 & 0.58 \pm 0.22\end{array}$




\section{IV.D. THE SEMINAL VESICLES}

\section{IV.D.1 Lymphocytes}

The pattern of staining of the antilymphocyte antibodies within the seminal vesicles was more or less similar to that of the ampulla of the vas. Lymphocytes were very few in number and were all essentially T-lymphocytes of the suppressor/cytotoxic phenotype. They were detected within the convoluted lining epithelium and within the lamina propria of the seminal vesicles (Figure IV.17). Table IV.11 shows the reactivity of the Leu 4, Leu $2 a$ and Leu $3 a$ antibodies within the seminal vesicles. No B-1ymphocytes were detected.

\section{IV.D.2 Macrophages}

No detectable reaction was obtained with any of the antimacrophage (Leu M3, 3.9 and 24) antibodies.

\section{IV.D.3 The Anti HLA-DR Reactive Cells}

HLA-DR positive cells were detected in the lamina propria but the lining epithelium did not show any staining. 


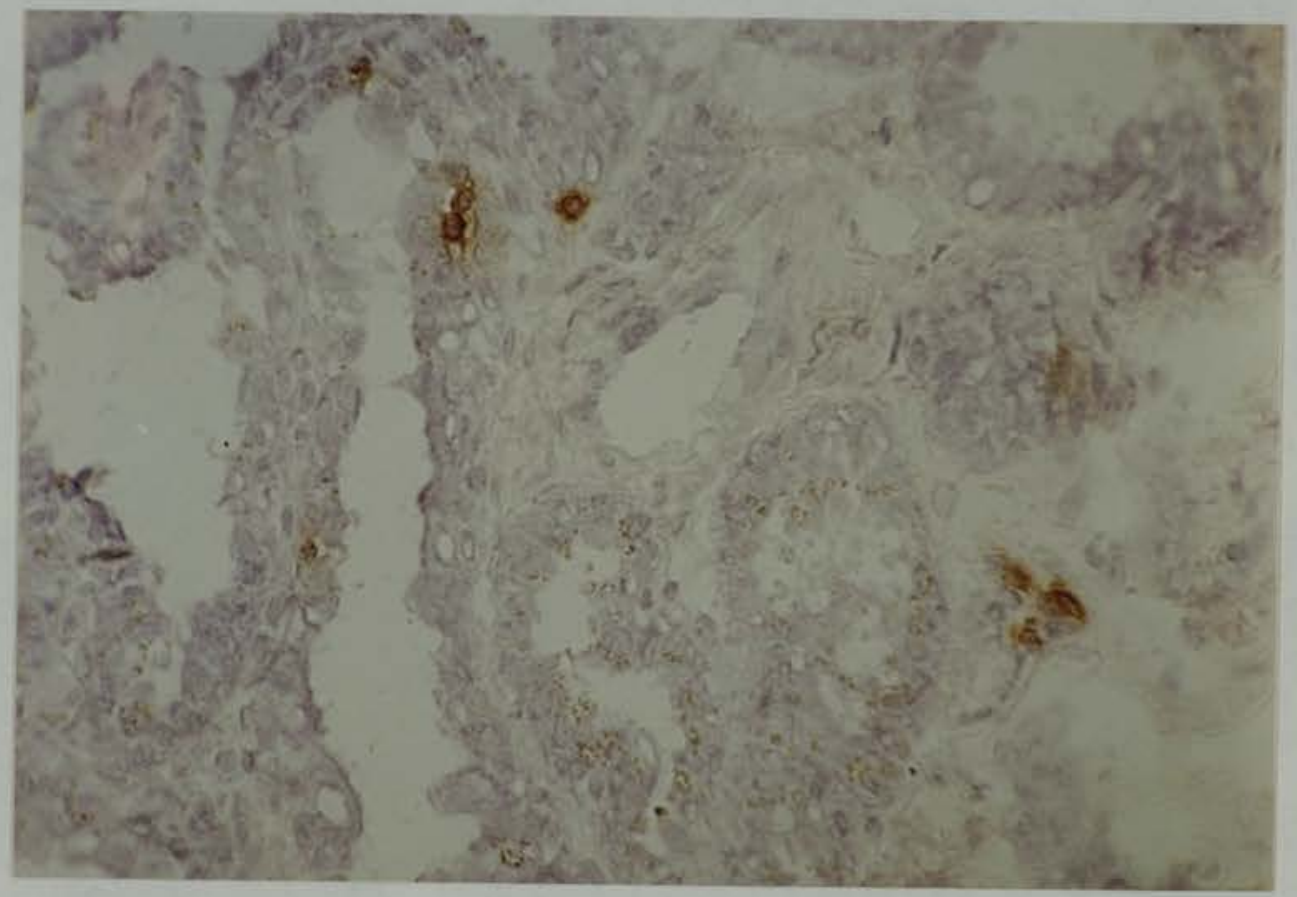

Figure IV.17: Immunoperoxidase staining of a cryostat section of seminal vesicles stained for suppressor/cytotoxic T-cells ( $x$ 160).

Note that these cells are few compared with those noted in the epididymis and vas deferens. 
TABLE IV.11:

T-LYMPHOCYTE SUBSETS IN THE SEMINAL VESICLES

\begin{tabular}{|c|c|c|c|}
\hline Donor & $\begin{array}{l}\text { Leu } 2 \mathrm{a}^{+} \text {cells } \\
\text { Leu } 4^{+} \text {cells ( } \mathrm{T} \text { suppressor/ } \\
\text { (A11 T-cells) cytotoxic) }\end{array}$ & $\begin{array}{l}\text { Leu } 3 \mathrm{a}^{+} \text {cells } \\
\text { ( } \mathrm{T} \text { helper/ } \\
\text { inducer) }\end{array}$ & $\begin{array}{l}\text { Leu } 2 a / \\
\text { Leu } 3 a\end{array}$ \\
\hline
\end{tabular}

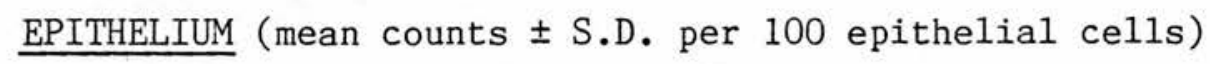

$\begin{array}{rllll}\text { D1 } & 2.48 & 1.52 & 0.68 & 2.23 \\ \text { D2 } & 2.20 & 1.60 & 0.49 & 3.26 \\ \text { D3 } & 2.95 & 3.01 & 0.62 & 4.85 \\ \text { D4 } & 2.36 & 2.15 & 0.62 & 3.46 \\ & & & & \\ \text { A11 donors } & 2.49 \pm 0.32 & 2.07 \pm 0.68 & 0.60 \pm 0.08 & 3.45 \pm 1.07\end{array}$

CONNECTIVE TISSUES OF THE LAMINA PROPRIA AND INTERSTITIUM

(mean counts \pm S.D. per $0.8 \mathrm{~mm}^{2}$ tissue $\mathrm{x} \mathrm{160)}$

$\begin{array}{lllll}\text { D1 } & 4.4 \pm 2.2 & 2.3 \pm 1.3 & 2.5 \pm 0.5 & 0.92 \\ \text { D2 } & 3.8 \pm 2.1 & 1.6 \pm 0.8 & 2.3 \pm 1.3 & 0.69 \\ \text { D3 } & 5.9 \pm 3.0 & 2.8 \pm 1.3 & 3.1 \pm 2.3 & 0.93 \\ \text { D4 } & 2.8 \pm 0.8 & 1.4 \pm 0.7 & 2.6 \pm 1.0 & 0.53\end{array}$

$\begin{array}{lllll}\text { All donors } & 4.22 \pm 1.29 & 2.02 \pm 0.64 & 2.62 \pm 0.34 & 0.76 \pm 0.19\end{array}$ 
IV.E THE PROSTATE

\section{IV.E.1 Normal Prostatic Tissue}

\section{IV.E.1.1 Lymphocytes}

Haematoxylin and eosin staining showed normal morphology with no hyperplastic or inflammatory changes in the eight specimens examined. The ducts and other acinar tissues were lined by columnar epithelium.

Immunoperoxidase staining of the cryostat sections of normal prostatic tissues revealed the presence of lymphocytes within the epithelial lining, within the lumina of the ducts in proximity to the corpora amylacea and in immediately periductal connective tissue septa. Within the epithelium, lymphocytes were almost all T-1ymphocytes (Leu $4^{+}$) and largely of the suppressor/cytotoxic phenotype (Leu $2 \mathrm{a}^{+}$) (Table IV.12a). They tended to lie close to the basement membrane but very occasionally some cells appeared further up in between the epithelial cells towards the lumen (Figure IV.18). The interstitial positively labelled cells were more abundant and were mainly T-cells of the helper/inducer phenotype (Leu $3 \mathrm{a}^{+}$) (Table IV.12b). They tended to be arranged in clumps rather than singly. Similarly there was a tendency to aggregation of lymphocytes within the interlobar septa of connective tissue. B-cell aggregations (Leu $14^{+}$) were also seen in the interstitial tissues. 
TABLE IV.12a:

T-LYMPHOCYTE SUBSETS IN NORMAL PROSTATIC EPITHELIUM (mean counts \pm S.D. per 100 epithelial cells)

\begin{tabular}{ccccc}
\hline Donor & $\begin{array}{c}\text { Leu } 4^{+} \text {cells } \\
\text { (A11 T-cells) }\end{array}$ & $\begin{array}{c}\text { Leu 2a } \mathrm{a}^{+} \text {cells } \\
\text { (T suppressor/ } \\
\text { cytotoxic) }\end{array}$ & $\begin{array}{c}\text { Leu } 3 \mathrm{a}^{+} \text {cells } \\
\text { (Telper/ } \\
\text { inducer) }\end{array}$ & $\begin{array}{c}\text { Cells ratio } \\
\text { Leu 2a/ } \\
\text { Leu 3a }\end{array}$ \\
\hline 1 & 15.36 & 8.01 & 3.30 & 2.42 \\
2 & 10.99 & 9.32 & 3.34 & 2.79 \\
3 & 11.65 & 9.09 & 3.22 & 2.82 \\
4 & 11.52 & 9.36 & 2.08 & 4.5 \\
5 & 10.34 & 9.51 & 2.20 & 4.3 \\
6 & 17.21 & 12.456 & 2.49 & 5.0 \\
7 & 11.78 & 9.69 & 1.88 & 5.15 \\
8 & 13.64 & 10.386 & 1.80 & 5.77 \\
A11 donors & $12.81 \pm 2.39$ & $9.72 \pm 1.28$ & $2.53 \pm 0.65$ & $4.09 \pm 1.25$ \\
\hline
\end{tabular}

TABLE IV.12b:

T-LYMPHOCYTE SUBSETS IN THE CONNECTIVE TISSUE STROMA OF NORMAL PROSTATE (mean counts \pm S.D. per $0.8 \mathrm{~mm}^{2}$ tissue $\mathrm{x} \mathrm{160}$ )

\begin{tabular}{|c|c|c|c|c|}
\hline Donor & $\begin{array}{l}\text { Leu } 4^{+} \text {cells) } \\
\text { (A11 T-cells) }\end{array}$ & $\begin{array}{l}\text { Leu } 2 \mathrm{a}^{+} \text {cells } \\
\text { ( } \mathrm{T} \text { suppressor } / \\
\text { cytotoxic) }\end{array}$ & $\begin{array}{l}\text { Leu } 3 \mathrm{a}^{+} \text {cells } \\
\text { ( } \mathrm{T} \text { helper/ } \\
\text { inducer) }\end{array}$ & $\begin{array}{l}\text { Cells ratio } \\
\text { Leu } 2 a / \\
\text { Leu } 3 a\end{array}$ \\
\hline $\begin{array}{l}1 \\
2 \\
3 \\
4 \\
5 \\
6 \\
7 \\
8\end{array}$ & $\begin{array}{l}6.5 \pm 3.5 \\
6.8 \pm 3.79 \\
8.8 \pm 5.18 \\
8.3 \pm 2.83 \\
12 \pm 2.1 \\
14.4 \pm 3.6 \\
7.5 \pm 1 \\
6.9 \pm 0.9\end{array}$ & $\begin{array}{l}3.3 \pm 2.00 \\
3.3 \pm 2.16 \\
4.7 \pm 3.12 \\
3.5 \pm 1.84 \\
3.8 \pm 0.6 \\
3.1 \pm 0.87 \\
1.5 \pm 0.5 \\
1.3 \pm 0.5\end{array}$ & $\begin{array}{l}5.7 \pm 2.83 \\
5.6 \pm 3.09 \\
5.9 \pm 3.95 \\
5.4 \pm 2.83 \\
7.4 \pm 1.7 \\
16.1 \pm 2.8 \\
6.0 \pm 0.8 \\
3.3 \pm 0.9\end{array}$ & $\begin{array}{l}0.57 \\
0.58 \\
0.79 \\
0.64 \\
0.51 \\
0.19 \\
0.25 \\
0.39\end{array}$ \\
\hline A11 donors & $8.91 \pm 2.83$ & $3.06 \pm 1.13$ & $6.92 \pm 3.87$ & $0.49 \pm 0.20$ \\
\hline
\end{tabular}



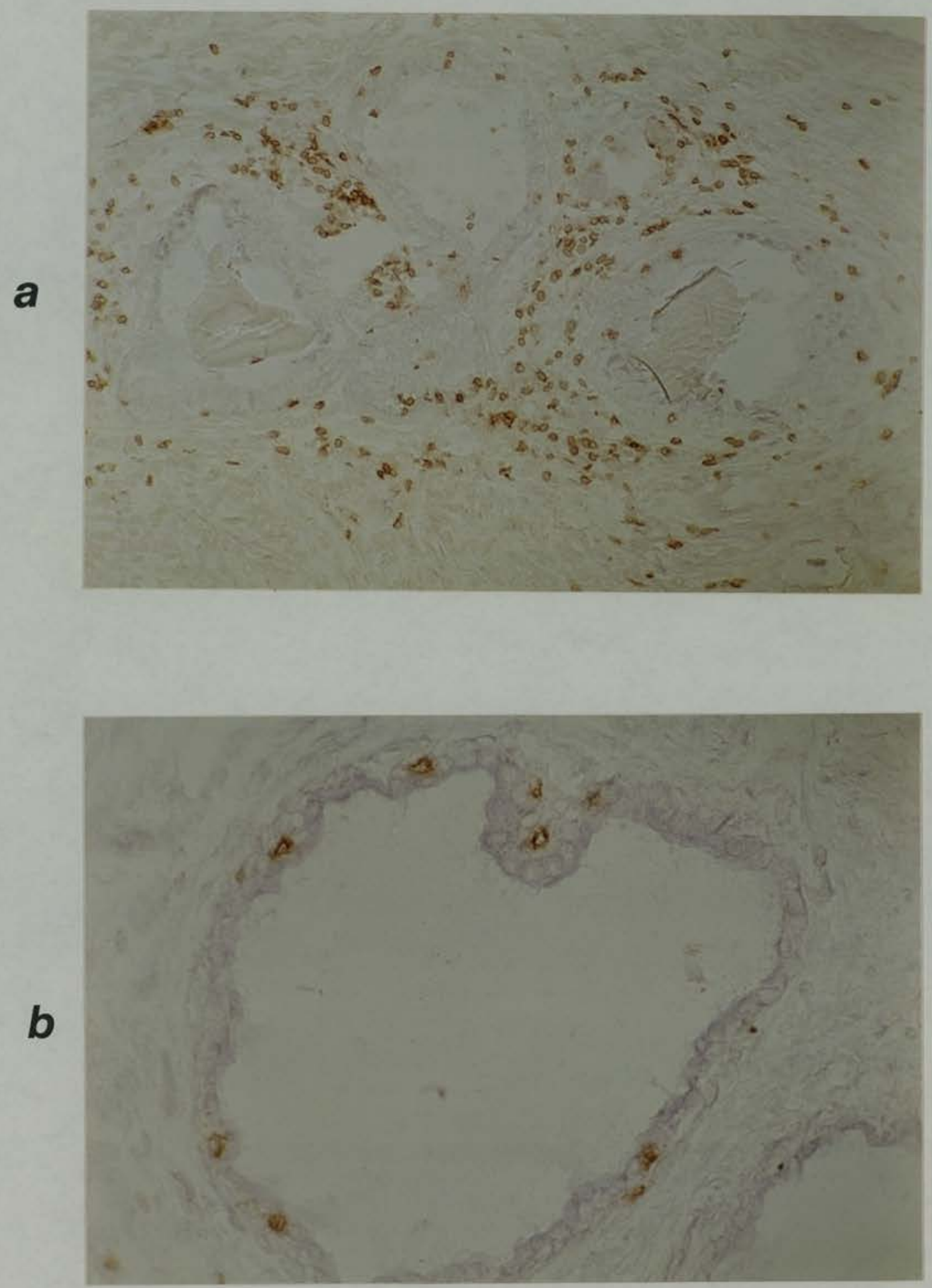

Figure IV.18: (a) Leu $4^{+}$cells (T-1ymphocytes) in normal prostate are seen in the lining epithelium, in the lumina of ducts and in the interstitium.

(b) Leu $2 \mathrm{a}^{+}$cells (suppressor/cytotoxic T-1ymphocytes) in the lining epithelium of prostatic acini.

(Cryostat sections, immunoperoxidase, x 160 \& x 320) 


\section{IV.E.1.2 Macrophages}

The Leu M3, 3.9 and 2.4 monoclonal antibodies stained a minority of cells within the lining epithelium and within the interstitial connective tissue stroma of the prostate (Figure IV.19).

\section{IV.E.1.3 Anti-HLA-DR reactive cells}

Cells reacting with the anti-HLA-DR antibody were consistently demonstrated along the basal layer of the lining epithelium of the prostatic acini and ducts and in very close proximity to the basement membrane (Figure IV.20). The rest of the epithelium was HLA-DR negative. Occasional HLA-DR positive cells were seen in the interstitial tissues.

\section{IV.E.1.4 Anti-Leu 7 reactivity to normal prostatic tissues}

The anti-Leu 7 antibody, which is believed to react with the natural killer (NK) cells, stained the prostatic epithelium. This anti-Leu 7 reactivity was localised in the cytoplasm of the ductal and acinar epithelium. However, some acini and ducts were not stained, i.e. the staining was heterogenic. The fibromuscular stroma did not stain (Figure IV.21). In contrast, the anti-Leu 11b antibody, which also reacts with the NK cells, did not show any reactivity with the normal prostatic epithelium.

It should be stressed that specific staining was obtained with anti-Leu 7 and anti-Leu 11 in the positive control sections (tonsils) while negative control sections showed no staining. 


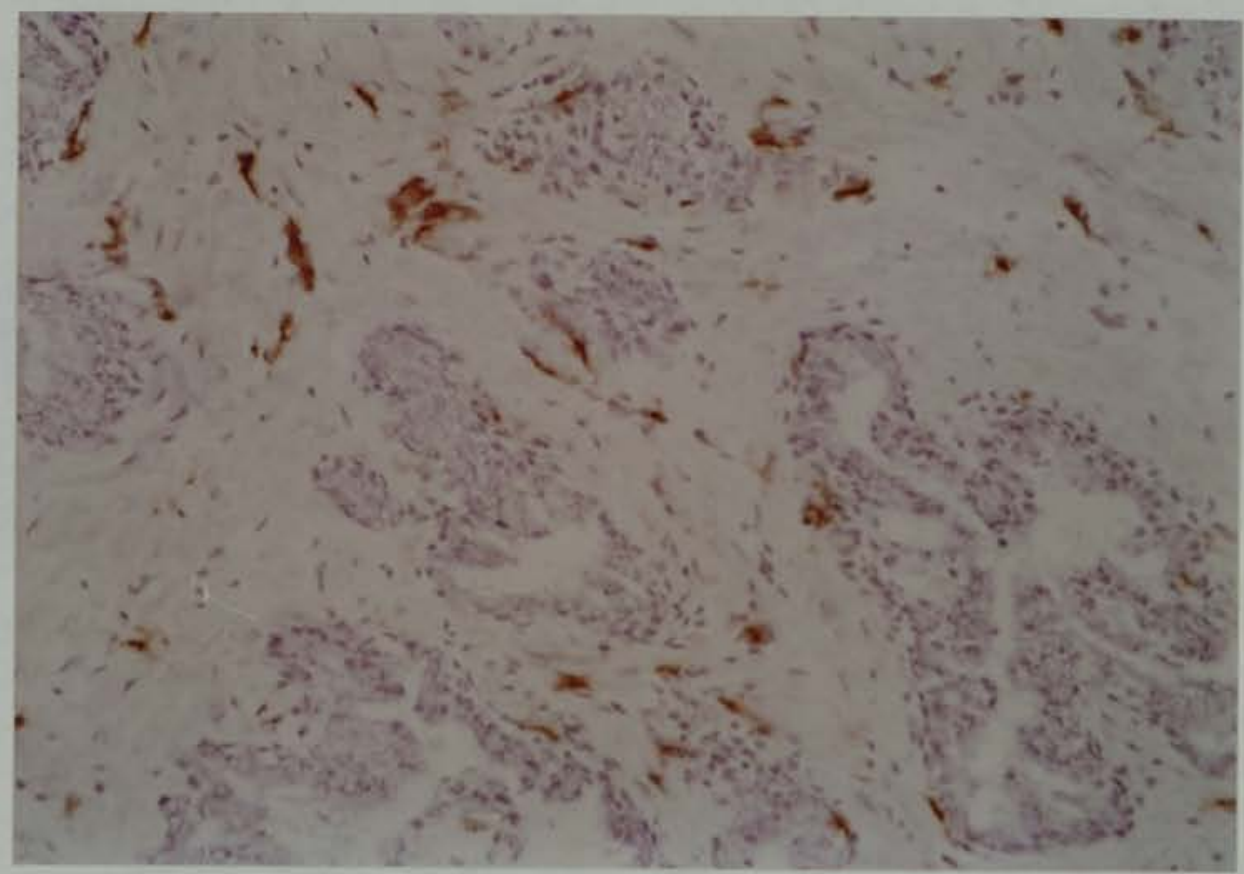

Figure IV.19: Leu $\mathrm{M3}^{+}$cells (macrophages) in normal prostate (Cryostat section, immunoperoxidase, $x$ 80)

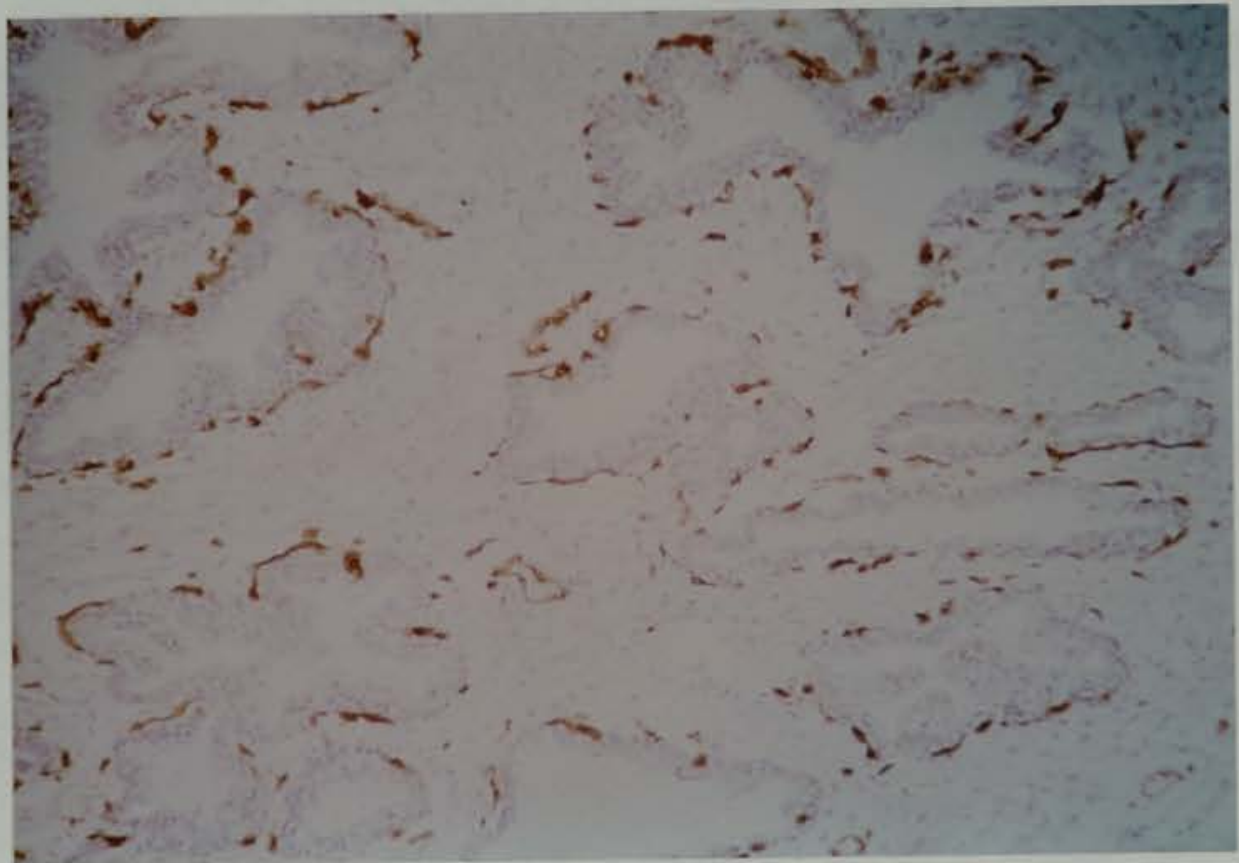

Figure IV.20: $\quad$ HLA-DR ${ }^{+}$cells in normal prostate.

(Cryostat section, immunoperoxidase, $\mathrm{x} 80$ )

Note the location of these cells in the basal layer of the fpithelium and that the epithelium is not stained. 


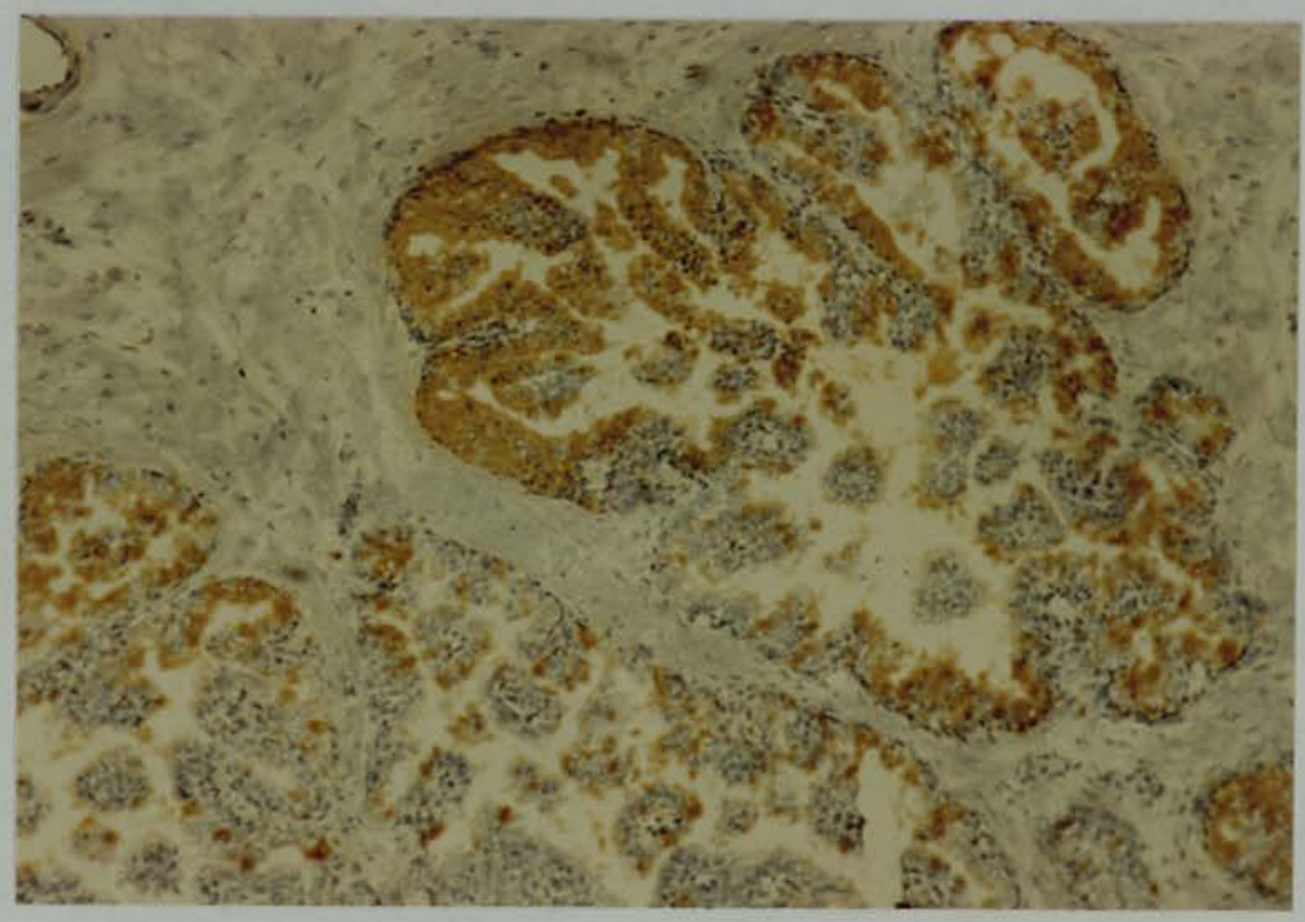

Figure IV.21: Immunoperoxidase staining of a cryostat section of prostate tissue stained with Leu 7 antibody. Note the staining of prostatic epithelium. ( $x$ 160) 


\section{IV.E.2 Benign Prostatic Hyperplasia Tissues}

\section{IV.E.2.1 Lymphocytes}

The hyperplastic prostates showed cystic dilatation of the glandular epithelium and thick fibrous septa. Around the prostatic acini there were accumulations of the positively stained T-lymphocytes (Leu $4^{+}$) which were surrounding almost concentrically the glands. Some of these Leu $4^{+}$cells were present within the lumen. The majority of T-lymphocytes within the prostatic epithelium exhibited the suppressor/cytotoxic phenotype, i.e. Leu $2 \mathrm{a}^{+}$. The helper/inducer T-cells (Leu $3 \mathrm{a}^{+}$) predominated in the connective tissue stroma (Figure IV.22). T-1ymphocyte counts within the epithelium were expressed as average numbers/100 epithelial cells and in interstitial tissues as average numbers/HPF $\left(0.8 \mathrm{~mm}^{2}\right.$ tissue). The mean counts of Leu $4^{+}$, Leu $2 \mathrm{a}^{+}$and Leu $3 \mathrm{a}^{+}$ cells and the mean Leu $2 \mathrm{a}$ /Leu 3 a ratios are shown in Tables IV.13a and b. There were no significant differences in Leu $4^{+}$, Leu $2 a^{+}$or Leu $3 \mathrm{a}^{+}$cell counts within the epithelium between the normal donors and the patients with benign prostatic hyperplasia (BPH). The highest level of Leu $4^{+}$cells (T-1ymphocytes) and Leu $2 a^{+}$cells (T-suppressor/cytotoxic cells) was observed in the patient with chronic prostatitis. In this patient activated T-cells (1L2-r ${ }^{+}$ cells) were also found within the epithelium and within the interstitial tissues (Figure IV.23a). In the interstitial tissues, the T-1ymphocyte counts in the patients with BPH were significantly higher than in normal donors, for Leu $4^{+}$cells $(P<0.001)$, Leu $2 \mathrm{a}^{+}$ 

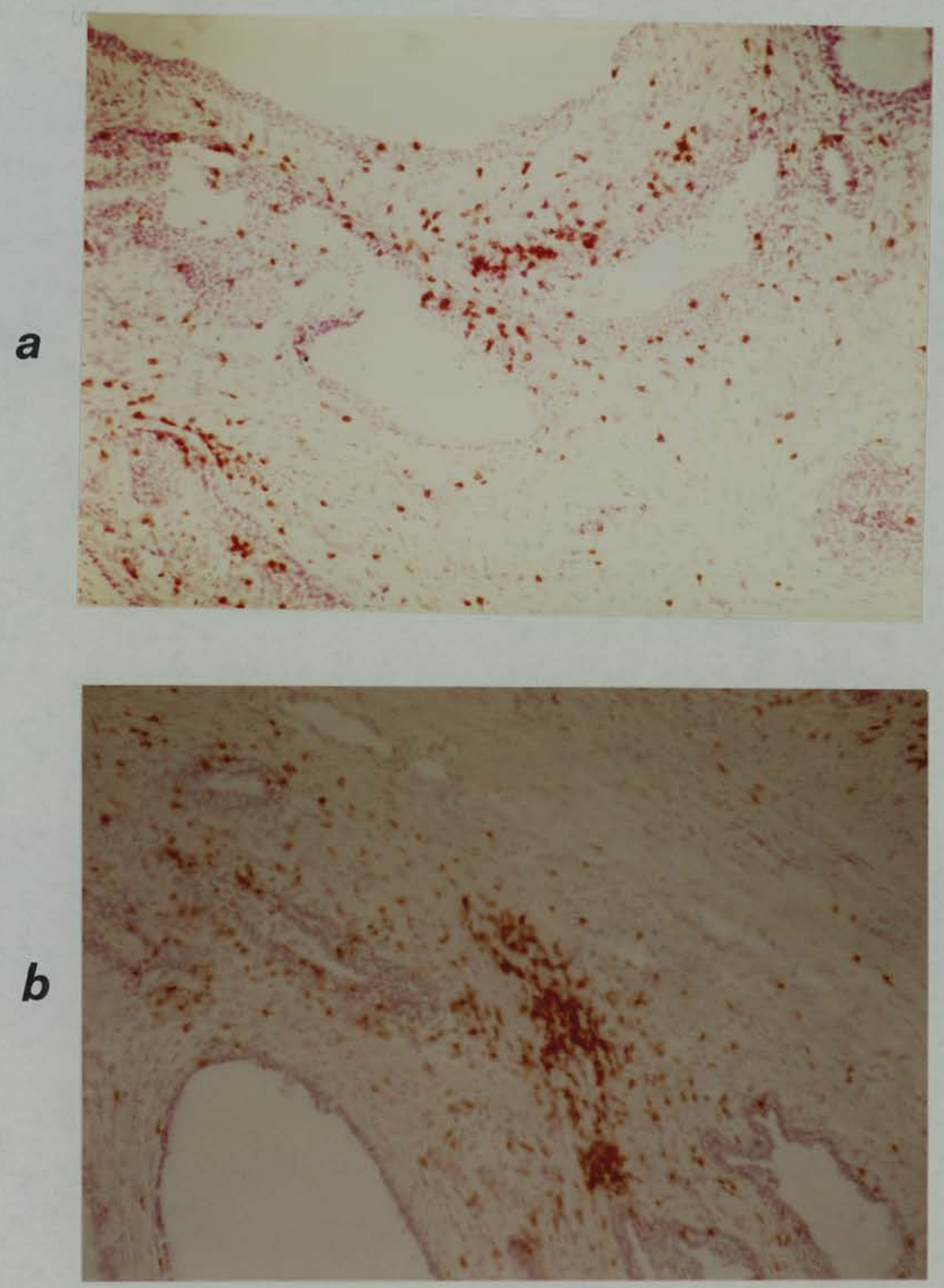

Figure IV.22: Immunoperoxidase staining of cryostat sections of BPH tissues stained for

(a) suppressor/cytotoxic T-cells (Leu $2 \mathrm{a}^{+}$)

(b) helper/inducer T-cells (Leu $3 \mathrm{a}^{+}$)

Note increased infiltration of the lining epithelium and the interstitial connective tissue with Leu $2 \mathrm{a}^{+}$and Leu $3 \mathrm{a}^{+}$cells respectively. $(x)$ 


\section{TABLE IV.13a:}

T-LYMPHOCYTE SUBSETS IN THE PROSTATIC EPITHELIUM IN BPH TISSUES (mean counts \pm S.D. per 100 epithelial cells)

\begin{tabular}{|c|c|c|c|c|}
\hline Patient & $\begin{array}{l}\text { Leu } 4^{+} \text {cells } \\
\text { (A11 T-cells) }\end{array}$ & $\begin{array}{c}\text { Leu } 2 \mathrm{a}^{+} \text {cells } \\
\text { ( } \mathrm{T} \text { suppressor } \\
\text { cytotoxic) }\end{array}$ & $\begin{array}{c}\text { Leu } 3 \mathrm{a}^{+} \text {cells } \\
\text { (T helper/ } \\
\text { inducer) }\end{array}$ & $\begin{array}{l}\text { Cells ratio } \\
\text { Leu } 2 a / \\
\text { Leu } 3 a\end{array}$ \\
\hline $\begin{array}{l}017862 \\
\text { WW } \\
324650 \\
385636 \\
070220 \\
375205 \\
284249 \\
017867\end{array}$ & $\begin{array}{l}21.22 \\
12.04 \\
10.88 \\
12.06 \\
13.50 \\
10.55 \\
15.13 \\
12.57\end{array}$ & $\begin{array}{r}13.70 \\
9.29 \\
7.70 \\
9.25 \\
11.36 \\
7.00 \\
9.41 \\
7.496\end{array}$ & $\begin{array}{l}4.32 \\
3.97 \\
3.18 \\
3.43 \\
1.90 \\
2.396 \\
4.39 \\
3.87\end{array}$ & $\begin{array}{l}3.171 \\
2.34 \\
2.42 \\
2.69 \\
5.98 \\
2.92 \\
2.14 \\
1.93\end{array}$ \\
\hline Al1 patients & $13.49 \pm 3.44$ & $9.4 \pm 2.22$ & $3.43 \pm 0.90$ & $2.94 \pm 1.28$ \\
\hline
\end{tabular}

TABLE 13b:

T-LYMPHOCYTE SUBSETS IN THE CONNECTIVE TISSUE STROMA OF BPH TISSUES (mean counts \pm S.D. per $0.8 \mathrm{~mm}^{2}$ tissue $\times 160$ )

\begin{tabular}{|c|c|c|c|c|}
\hline Patient & $\begin{array}{l}\text { Leu } 4^{+} \text {cells } \\
\text { (A11 T-cells) }\end{array}$ & $\begin{array}{c}\text { Leu } 2 \mathrm{a}^{+} \text {cells } \\
\text { ( } \mathrm{T} \text { suppressor } / \\
\text { cytotoxic) }\end{array}$ & $\begin{array}{c}\text { Leu } 3 \mathrm{a}^{+} \text {cells } \\
\text { ( } \mathrm{T} \text { helper/ } \\
\text { inducer) }\end{array}$ & $\begin{array}{l}\text { Cells ratio } \\
\text { Leu } 2 \mathrm{a} / \\
\text { Leu } 3 \mathrm{a}\end{array}$ \\
\hline $\begin{array}{l}113855 \\
324650 \\
\text { WW } \\
385636 \\
070220 \\
375205 \\
284249 \\
017867\end{array}$ & $\begin{array}{l}30.6 \pm 9.3 \\
14.8 \pm 4.10 \\
32.3 \pm 14.49 \\
32.6 \pm 14.58 \\
23.7 \pm 6.92 \\
26.4 \pm 9.38 \\
17.9 \pm 6.15 \\
15.7 \pm 7.10\end{array}$ & $\begin{array}{r}12.2 \pm 3.32 \\
5.6 \pm 1.95 \\
6.1 \pm 2.50 \\
6.5 \pm 3.65 \\
6.2 \pm 2.82 \\
7.9 \pm 3.60 \\
3.9 \pm 1.44 \\
3.1 \pm 0.99\end{array}$ & $\begin{array}{l}24.5 \pm 9.4 \\
13.5 \pm 4.0 \\
28.3 \pm 15.12 \\
29.6 \pm 9.32 \\
23.4 \pm 9.70 \\
13.3 \pm 3.83 \\
11.0 \pm 2.0 \\
5.4 \pm 2.41\end{array}$ & $\begin{array}{l}0.49 \\
0.47 \\
0.21 \\
0.21 \\
0.26 \\
0.59 \\
0.35 \\
0.57\end{array}$ \\
\hline Al1 patients & $24.25 \pm 7.39$ & $6.43 \pm 2.77$ & $18.62 \pm 8.93$ & $0.37 \pm 0.1$ \\
\hline
\end{tabular}



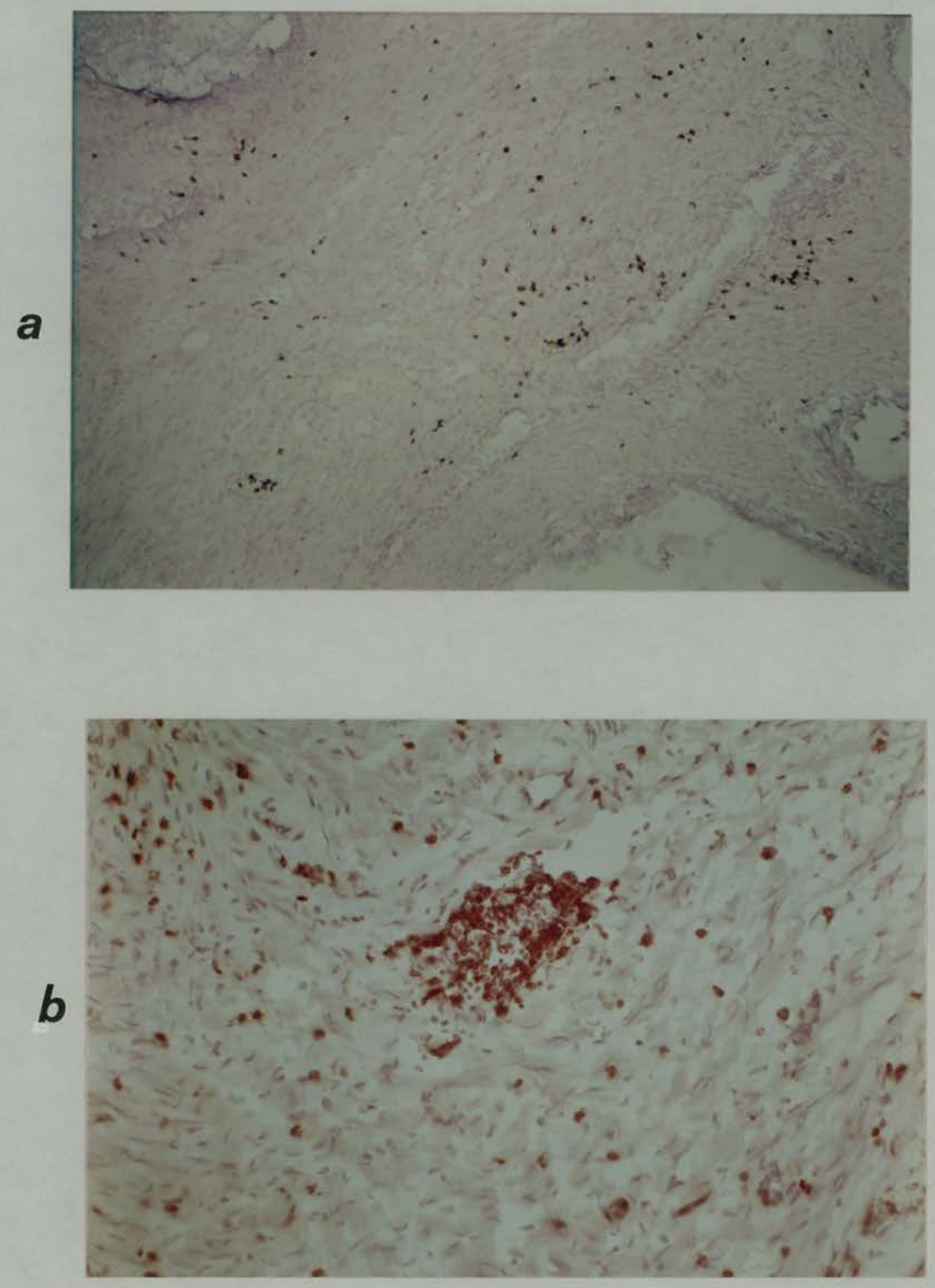

Figure IV.23: Immunoperoxidase staining of cryostat sections of prostatic tissue, obtained from a 54 year old patient with $\mathrm{BPH}$ and chronic prostatitis, stained for: (a) activated T-cells (1L2-r ${ }^{+}$)

(b) B-1ymphocytes (Leu $14^{+}$)

(Cryostat sections, immunoperoxidase, x 160) 
cells $(P<0.01)$ and Leu $3 a^{+} \operatorname{cells}(P<0.05)$. Figures IV.24 and 25 show plots of the Leu $2 \mathrm{a}^{+}$cell counts against Leu $3 \mathrm{a}^{+}$cell counts for individual subjects in both the normal donor and the patient groups.

B-lymphocytes (Leu $14^{+}$cells) were demonstrated as small follicles within the connective tissue stroma and were more frequent than those in the normal prostatic tissues (Figure IV.23b).

\section{IV.E.2.2 NK-ce11s}

The reactivity of the anti-Leu 7 antibody (HNK-1) in tissues with $\mathrm{BPH}$ was similar to that in the tissues from normal donors, i.e. the Leu 7 antibody reacted with the prostatic epithelium of $\mathrm{BPH}$.

The Leu $11 \mathrm{~b}$ antibody showed no reactivity with the prostatic epithelium. On the contrary, it stained single cells within the epithelium and within the connective tissue stroma. The Leu $11 b^{+}$cells were more numerous within the connective tissue stroma (Figure IV.26).

\section{IV.E.2.3 Macrophages and anti-HLA-DR reactive cells}

The reactivity of the antimacrophage and anti-HLA-DR antibodies showed no obvious difference from that in the normal tissue. 


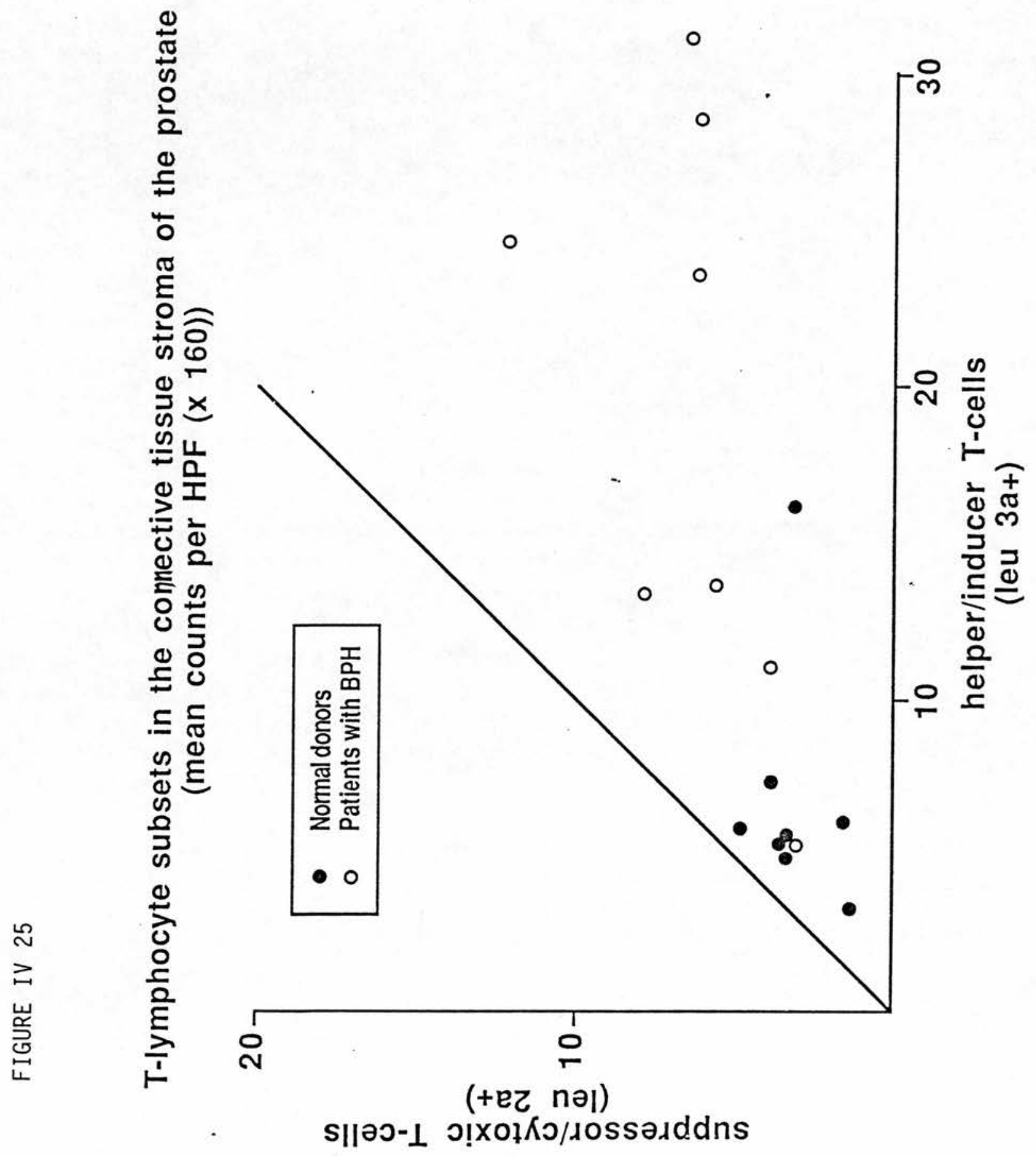


T-lymphocyte subsets in prostatic epithelium (mean counts per 100 epithelial cells)

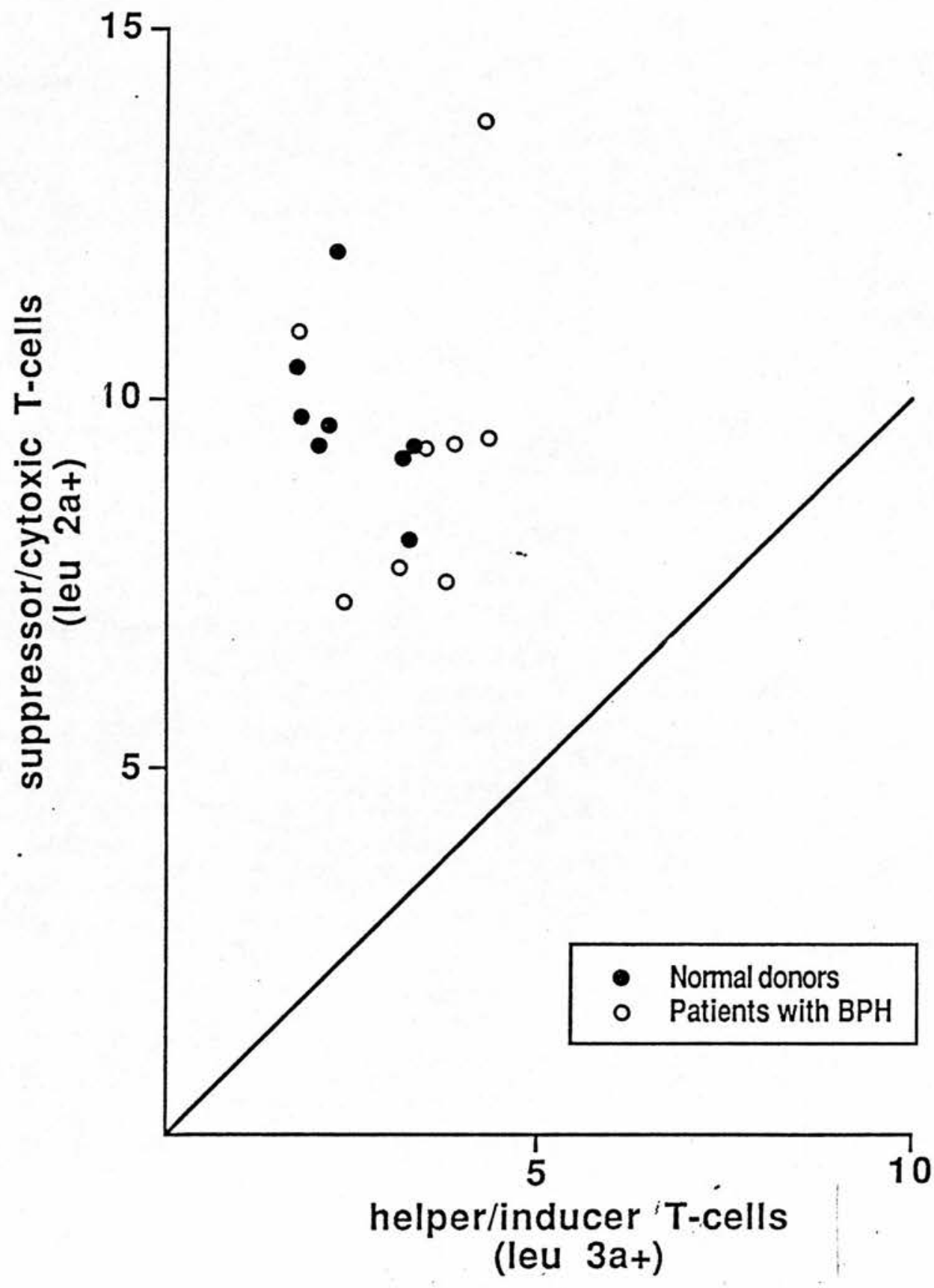



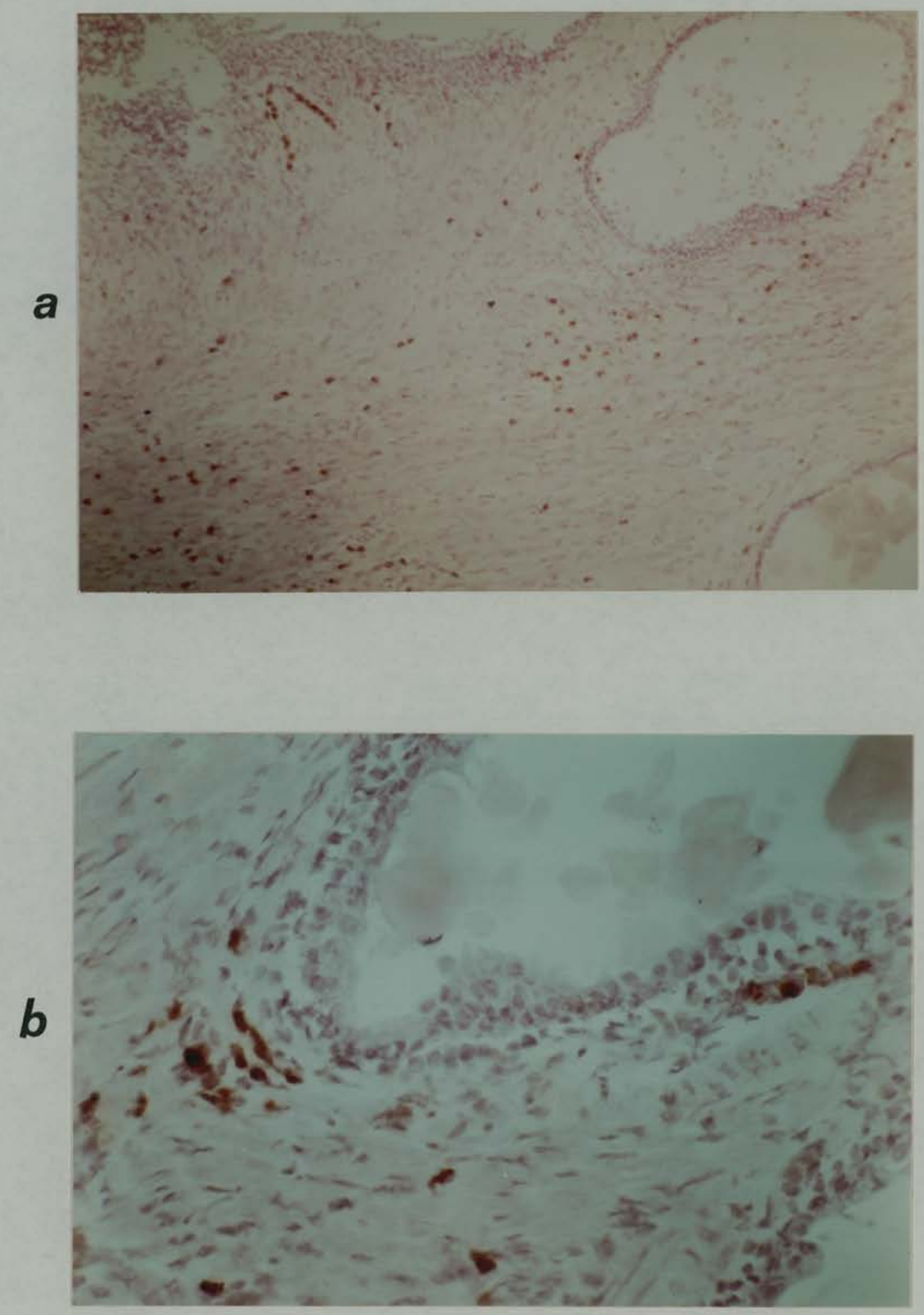

Figure IV.26: Immunoperoxidase staining of cryostat sections of BPH tissue with Leu $11 \mathrm{~b}$ antibody.

Note that this antibody (unlike Leu 7) did not stain prostatic epithelium and Leu $11 \mathrm{~b}^{+}$(NK) cells are located mainly in the interstitial connective tissue. ( $a=x 80 ; \quad b=x 160)$ 


\section{IV.F. T-LYMPHOCYTE SUBSETS IN THE MALE GENITAL TRACT:}

\section{SUMMARY OF RESULTS}

Lymphocyte subpopulations have a specific pattern of distribution in various parts of the male genital tract. In all normal tissues, apart from the peripheral testis where no lymphocytes were identified, T-1ymphocytes (Leu $4^{+}$) were found to be the predominant cell type. These cells were largely of the suppressor cytotoxic phenotype (Leu $2 \mathrm{a}^{+}$) and were more abundant in between the epithelial cells of the rete testis, epididymis, vas deferens, seminal vesicles and prostatic ducts and acini. Table IV.14 and Figures IV. 27 - 30 show the distribution of T-cell subsets within the epithelia of the male genital tract organs. Cells of the helper/inducer phenotype (Leu $3 a^{+}$) were identified mainly within the interstitial connective tissue. Very few B-lymphocytes (Leu $14^{+}$) were identified and these were mainly in the connective tissue stroma of the prostate. In each organ the ratio of suppressor/cytotoxic T-cells to the helper/inducer T-cells was consistent. Derangement of the pattern of distribution of lymphocyte subsets and alterations in the T-suppressor/cytotoxic to the T-helper/inducer ratios were observed in subfertile males.

Testicular biopsies from patients with testicular obstruction showed marked infiltration with T-1ymphocytes. The helper/inducer T-cells (Leu $3 \mathrm{a}^{+}$) predominated in biopsies from unilaterally obstructed and vasectomised patients. The suppressor/ cytotoxic T-cells predominated in biopsies from patients with obstructive azoospermia. Epididymal biopsies showed a decrease in 
the suppressor/cytotoxic T-cells and an increase in the helper/ inducer T-cells. The patient with chronic prostatitis showed high levels of T-cells, some of which were in an activated state as indicated by their expression to the 1L2-r antigens. 


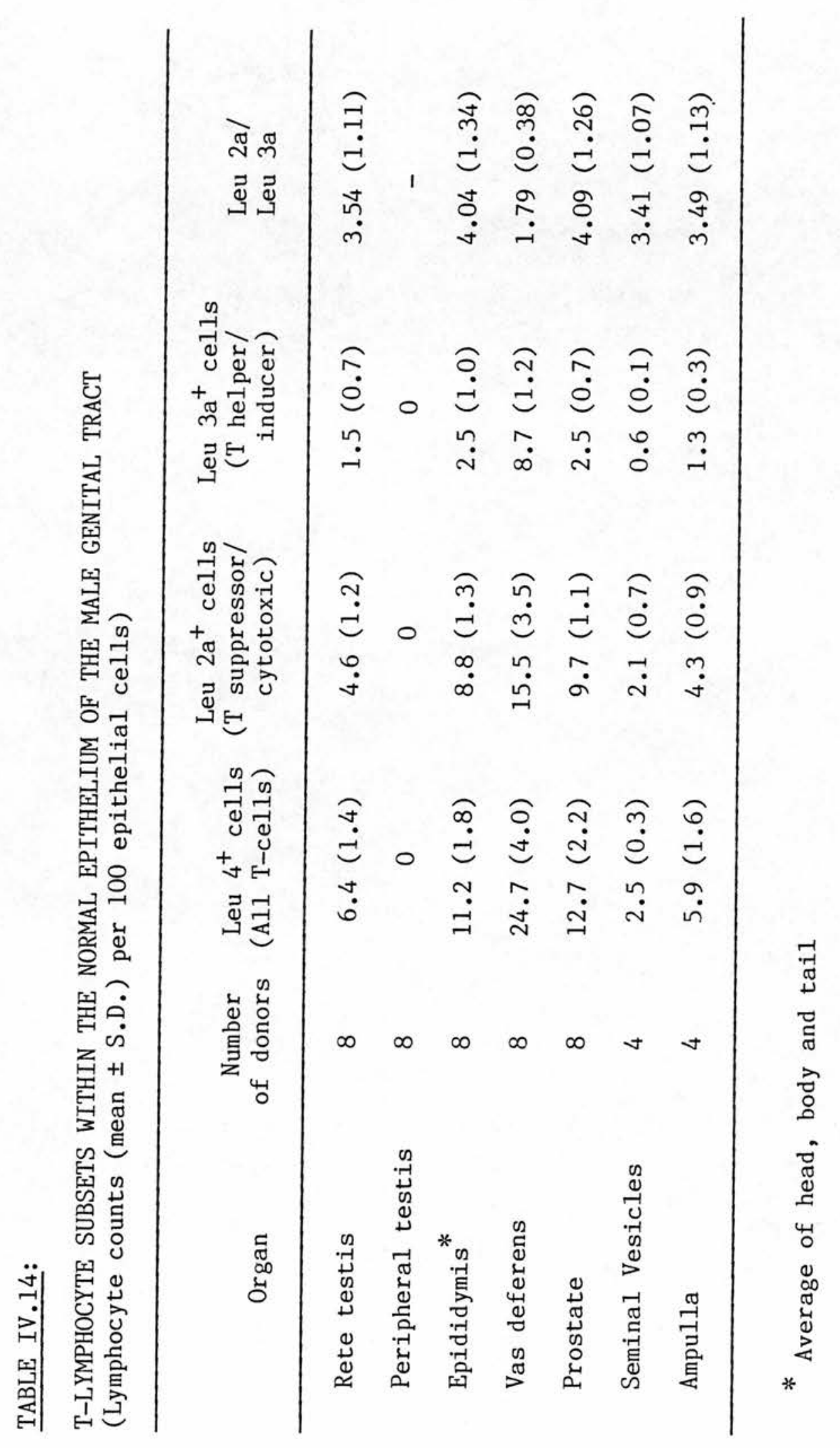


T-lymphocytes within the epithelium

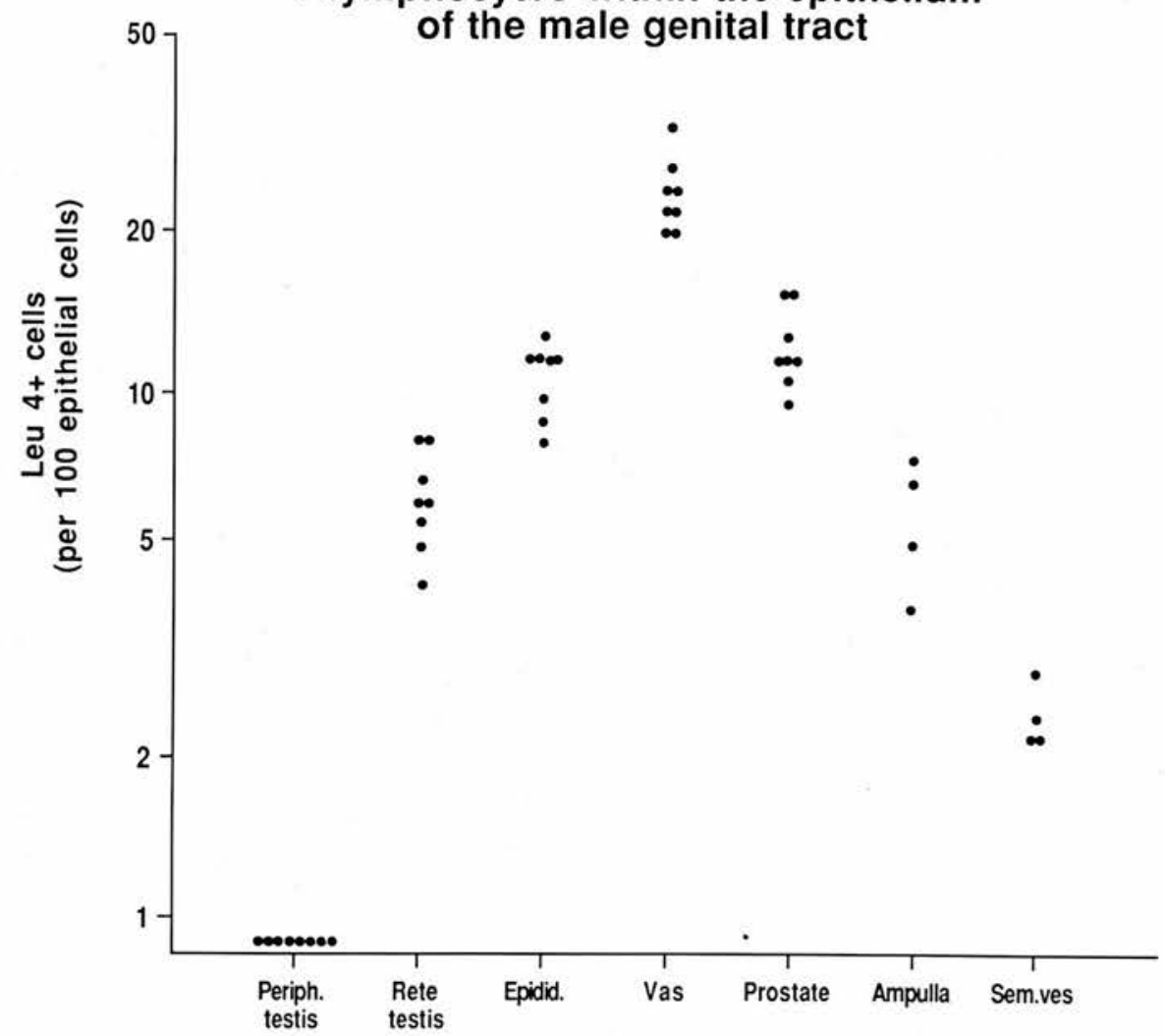

T-suppressor/cytoxic cells within the epithelium of the male genital tract

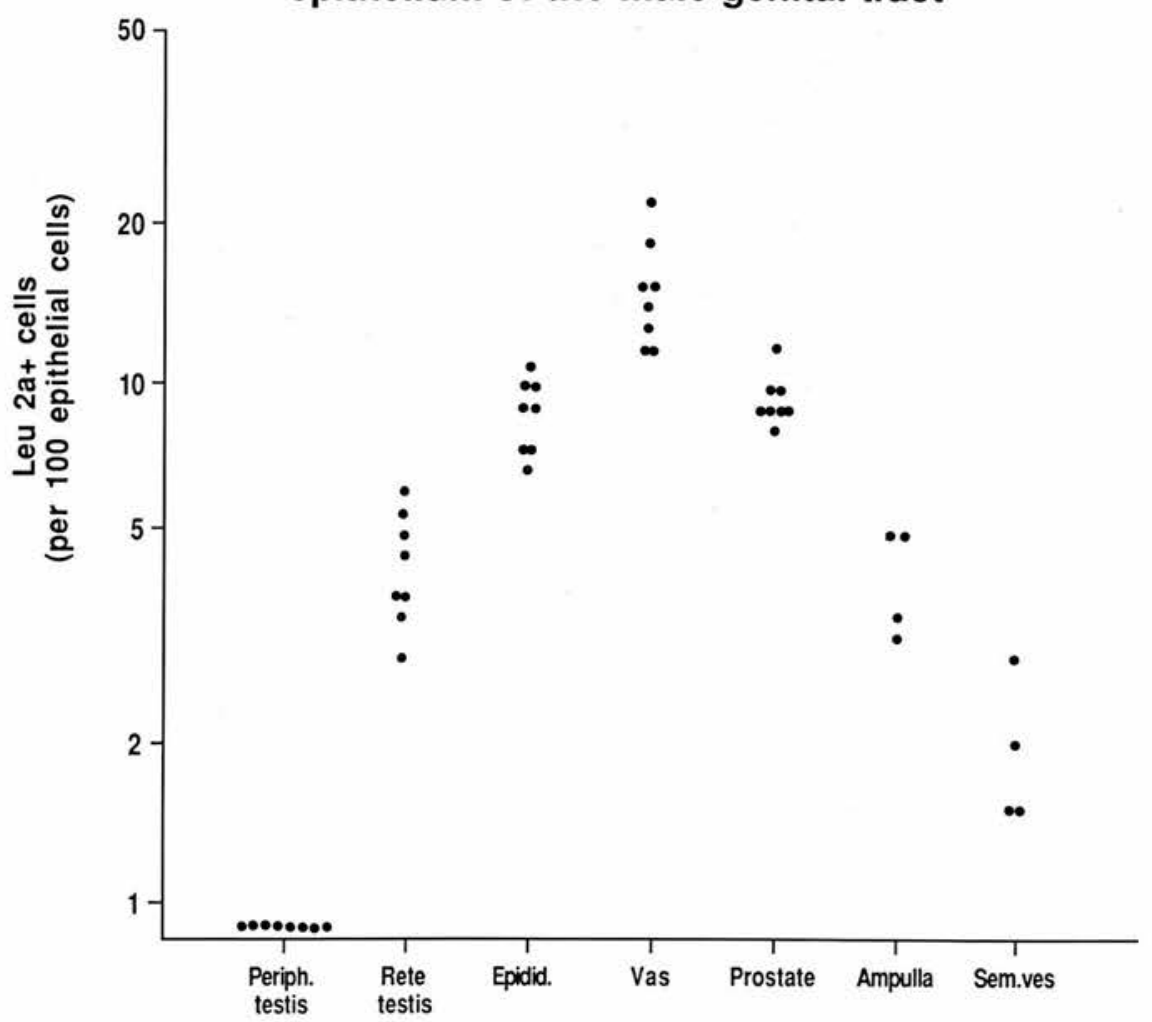

Figure IV.27 top.

Figure IV.28 bottom.

The data are shown on a logarithmic scale. 


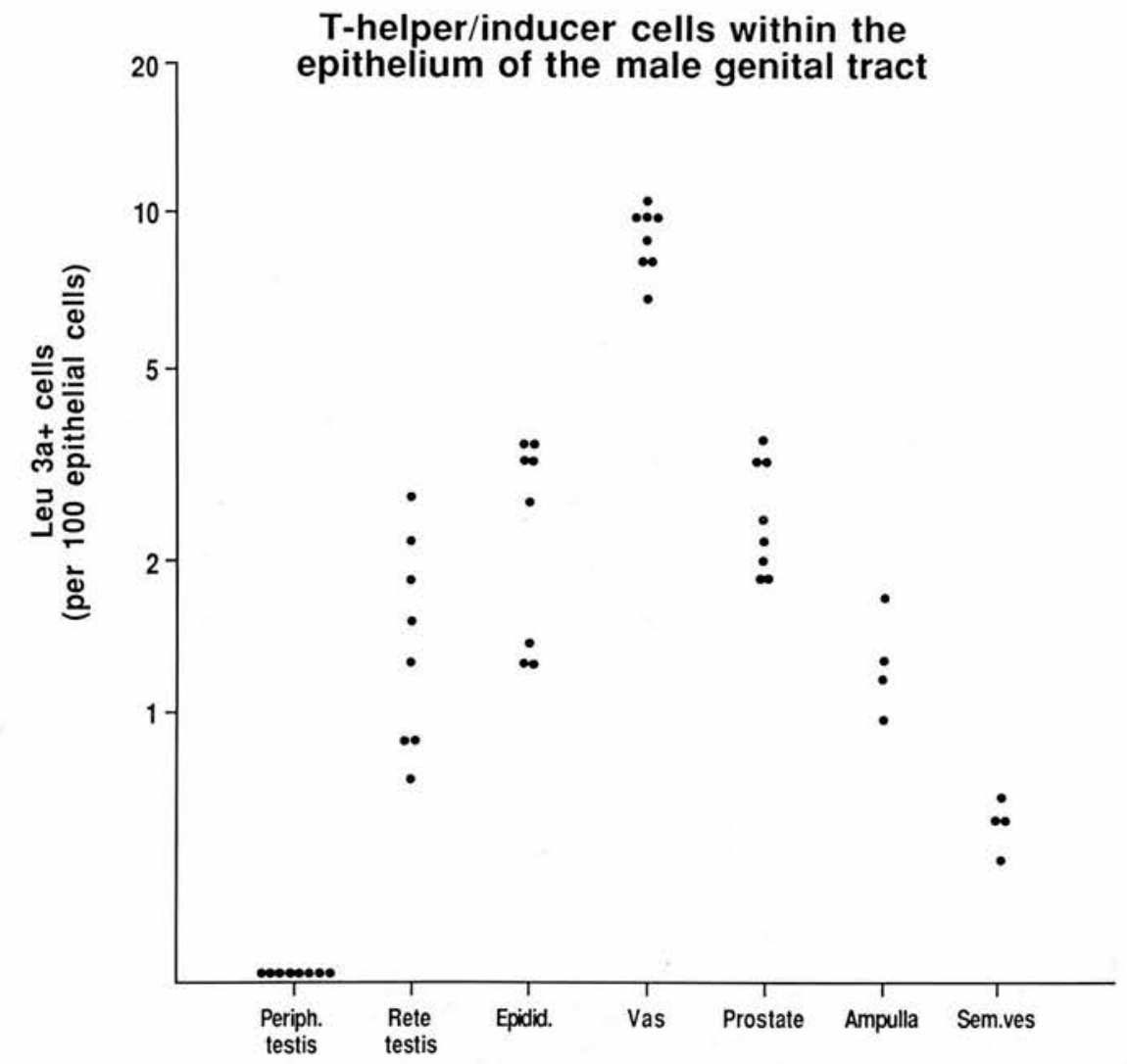

The ratio of T-suppressor/cytotoxic to T-helper/inducer cells within the epithelium of the male genital tract

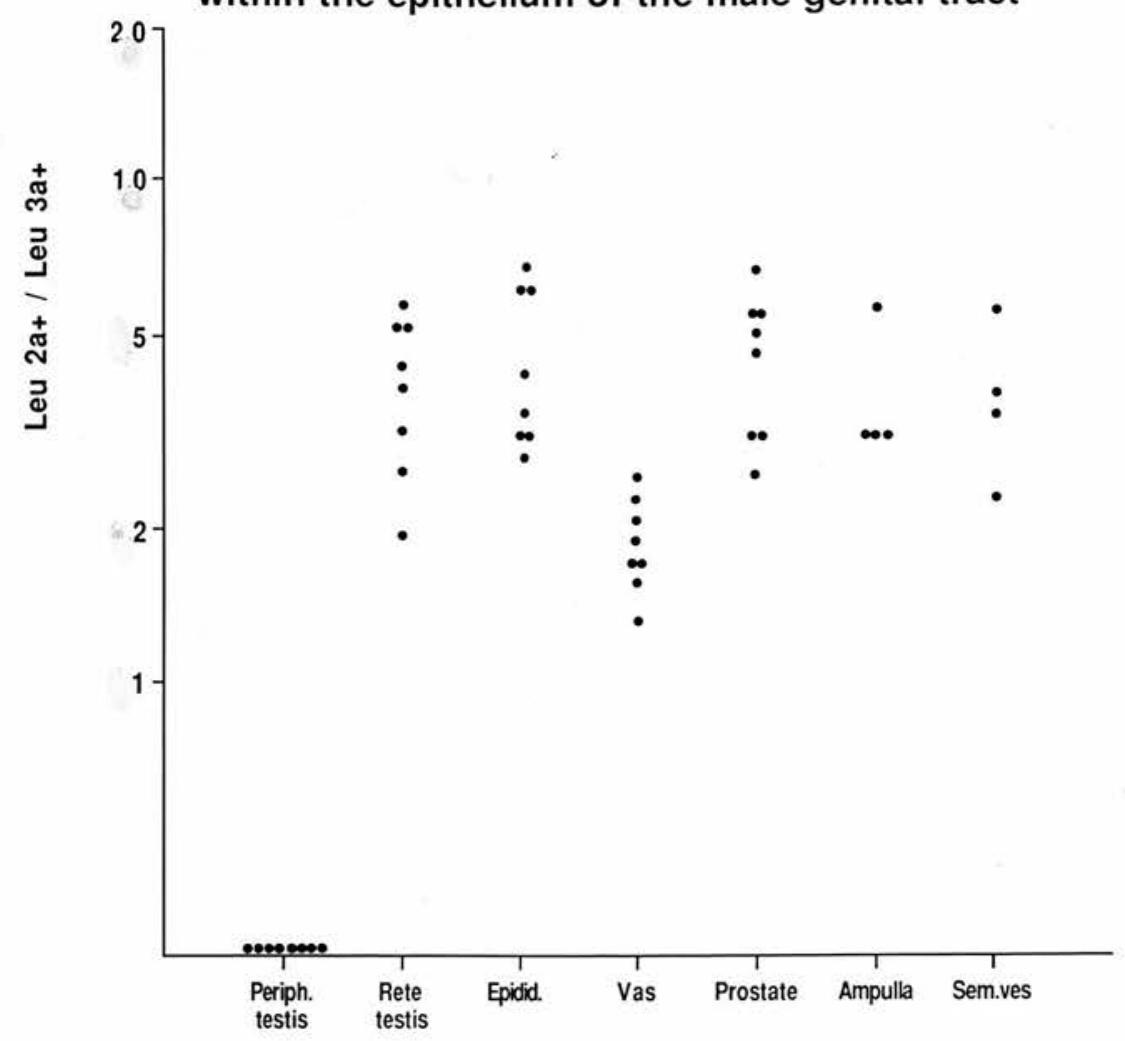

The data are shown on a logarithmic scale. 
IV.G DISCUSSION

\section{IV.G.1 Lymphocytes}

Lymphocytes have been reported in the epithelium of the intestinal tract (Ferguson, 1977; Selby et al., 1981); the respiratory tract (Bienenstock et al., 1973) and the reproductive tract (Dym and Romrell, 1975). It has been suggested that these lymphocytes are more likely to be present in every mucosal epithelial layer forming a common mucosal immunological system (Bienenstock et al., 1980).

Lymphocytes have been investigated in rat epididymis and attempts to identify them by light and electron microscopy have produced descriptions of "halo cells" (Reid and Cleland, 1957), "lymphocyte-1ike cells" (Hamilton, 1972), "agranular leucocytes" (Hoffer et al., 1973) and "intra-epithelial 1ymphocytes" (Dym and Romre11, 1975). Recent1y, ultrastructural and immunohistological evidence for the presence of lymphocytes in human epididymis has been reported (Holstein, 1983; Ritchie et al., 1984a).

In the present study, using specific monoclonal antibodies, we have demonstrated 1ymphocyte subpopulations and macrophages and defined their anatomical locations within the tissues of the male genital tract. Lymphocytes were almost always T-cells and they tended to be concentrated in large numbers in the epididymis, vas deferens and the prostate. They were few in numbers in the seminal vesicles. B-lymphocytes were rarely encountered in this study and 
were demonstrated mainly in the connective tissue stroma of the prostate.

T-1ymphocytes exhibited a compartmentalised distribution. Within the lining epithelium, lymphocytes of the suppressor/ cytotoxic phenotype were the major component. This was observed at all levels of the genital tract. This cell type was also preponderant in the subepithelial connective tissue (the lamina propria) of the epididymis, vas deferens, and seminal vesicles. In contrast, the helper-inducer T-lymphocytes were more abundant in the interstitial connective tissues.

The demonstration of resident populations of T-1ymphocyte subsets, within the tissues of the male genital tract, with a preponderance of the suppressor/cytotoxic subset in the epithelial lining suggests an important immunological barrier with distinct immunological function. It has been postulated that the helper/ inducer T-subset induces B-cell proliferation, differentiation and immunoglobulin production and helps in the development of the cytotoxic killer cells in cell-mediated lympholysis, i.e. they provide helper (inducer) function in $\mathrm{T}-\mathrm{T}, \mathrm{T}-\mathrm{B}$ and $\mathrm{T}$-macrophage interaction, while the suppressor/cytotoxic subset of T-cells was shown to suppress B-cell immunoglobulin production either by soluble mediators or through direct $\mathrm{T}-\mathrm{T}$ or $\mathrm{T}-\mathrm{B}$ interaction (Reinherz and Schlossman, 1980b). As previously suggested by Ritchie et al. (1984a), it is most likely that the predominant suppressor/cytotoxic $\mathrm{T}$-cells in the epithelial lining of the male genital tract form a major immunological barrier which normally prevents the development of autohumoral or cellular immune responses to sperm and other specific antigens. Human intestinal intraepithelial lymphocytes 
have been shown to possess immunoregulatory activity when added to co-cultures of peripheral blood lymphocytes in a pokeweed-mitogen driven immunoglobulin synthesis (Greenwood et al., 1983). The 1ymphocytes were largely T-cells of the suppressor phenotype, and in common with other suppressor effector cells (in contrast with cytotoxic effectors) responded poorly to mitogens and did not mediate cytotoxic functions in vitro (Clement et al., 1984).

The pattern of distribution of this suppressor barrier seems to be consistent with the functional properties of each organ. The absence of detectable lymphocytes in the normal peripheral testis may be explained by the presence of the blood-testis barrier which is formed mainly by the tight junctions of the Sertoli cells (Johnson, 1973). This barrier may not only serve to isolate and protect the maturing germ cells from potentially harmful blood-borne substances, but may be equally important in preventing sperm antigens from entering the circulation and eliciting an immune response. In other words, this blood-testis barrier ensures that the body's immunological system does not recognize differentiation antigens on haploid germ cells as non self. Under such circumstances germ cells may not require the protection that such suppressor 1ymphocytes would afford.

In contrast, T-1ymphocytes were norma11y found in these parts of the excurrent genital duct system (i.e. in the rete testis, epididymis and vas deferens) where the blood-testis barrier is weak or absent (Johnson, 1973), occurring only at the level of the Sertoli cells and where the sperm and/or sperm components are in contact directly with the outside environment. 
The epididymis absorbs most of the fluid secreted by the testis and the products of sperm breakdown (Jenkins et al., 1978). Approximately half the spermatozoa that enter the head of the epididymis disintegrate before reaching the cauda region which stores mature spermatozoa (Fabbrini and Hafez, 1980). The vas deferens plays an important role, not only in sperm transport but also in the maintenance of epididymal sperm reserve.

Freund and Davis (1969) and Overstreet (1986) reported that $60-70 \%$ of the spermatozoa within a normal ejaculate come from the epididymis and the proximal part of the vas deferens. The ampulla of the vas also stores sperm. Under normal physiological conditions and during sexual rest, large numbers of the stored spermatozoa often disintegrate, leading to considerable absorption of fluid and possibly of products of sperm degradation. Since it is well established that human sperm are autoantigenic (Mancini, 1974), the absorption of sperm breakdown products may initiate immune responses against self, i.e. autoimmunity. However, the existence of a network of suppressor T-cells within the epithelium and the subepithelial connective tissue of the rete testis, epididymis and vas deferens could limit self reactivity to testicular and/or sperm antigens under normal, non pathological circumstances.

There is substantial evidence implicating suppressor T-cells in inducing tolerance against self antigens (Richaman et al., 1978; Cowing, 1985). T-cell tolerance induction was also shown to be Class II MHC (major histocompatibility complex) restricted (Lowy et a1., 1984). We noted, in the present study, definite expression of the HLA-DR antigens, Class II molecule of the MHC, on the actual 
epithelia of the rete testis, epididymis and vas deferens. It has been suggested that the expression of Class II MHC antigens on non-lymphoid organs may induce tolerance against self antigens, protecting self against both exogenous factors and autoimmunity under normal circumstances (Iwatani et al., 1985). Furthermore, the HLA-DR positive epithelial cells may act as antigen presenting cells, resulting in the induction of suppressor T-cells. Bland and Warren (1986) have demonstrated antigen presentation by $\mathrm{Ia}^{+}$ (DR-1ike) intestinal columnar epithelial cells and presented evidence suggesting that antigen presentation by gut epithelial cells does result in the induction of immunoregulatory T-suppressor cells.

The finding that the epithelium of the seminal vesicles contains few suppressor T-cells is also consistent with its functions. The seminal vesicles are not a storage depot for spermatozoa and the secretions of the seminal vesicles are only in contact with the spermatozoa at the moment of ejaculation (Clavert et a1., 1985), i.e. there is little or no absorption of sperm antigens. This also may explain why the seminal vesicle epithelium is HLA-DR negative. However, antigenic properties of the male sex accessory glands, the prostate and the seminal vesicles, have been reported (Flocks et al., 1960; Shulman and Orsini, 1970).

The observations of Rao and Sadri (1959), Grant and Everall (1963) and Barnes et al. (1965) that seminal fluid and prostatic extracts contain identical antigens, provide clues as to the significance of the antigens in this organ for immunological fertility disorders. This may explain in part the significance of the predominant suppressor/cytotoxic T-cells in the prostate. A 
potential consequence of this suppressor barrier in the prostate is that infections and inflammation are commonly encountered in young men. Inflammation may result in occlusion of the ejaculatory ducts and absorption of prostatic and sperm antigens. Alternatively, the elicitation of a localised genital tract immune response to pathogens may lead to the formation of bacterial membrane carbohydrate-directed antibodies that cross-react with carbohydrates on the sperm surface (Sarkar, 1974). The demonstration of B-1ymphocytes in the interstitial connective tissue of the prostate may account for the source and level of local secretion of immunoglobulins.

Several studies have suggested an association between sperm antibodies and epididymitis (Fjallbrant, 1965) and prostate vesiculitis (Fjallbrant and Obrant, 1968; Quesada et al., 1968; Witkin and Toth, 1983). In addition a higher incidence of prostatitis was noted in men with sperm antibodies than in their control group (Fjallbrant and Nilsson, 1977). Furthermore, in longitudinal studies in males with acute and chronic bacterial prostatitis, systemic and local humoral immune responses have been found (Shortliffe et al., 1981).

It has been demonstrated that antibacterial protection depends on both 1ymphocytes and macrophages (Mackaness, 1964; Kaufmann, 1984). Extracellular bacteria can be eliminated by phagocytes (granulocytes) and this process is amplified by opsonizing antibodies. Thus protective immunity against these organisms is mediated by B-cell-derived antibodies. Facultative intracellular bacteria, however, can survive and multiply within normal phagocytes and antibodies have little or no protective 
effects. Acquired resistance against these pathogens depends on specific $\mathrm{T}$-cells which activate macrophages harbouring bacteria for bacteriostatic and bactericidal activity.

Selective stimulation of $\mathrm{T}$-cell subsets is controlled by gene products of the MHC. Association of antigenic epitopes with Class I molecules will stimulate cytotoxic T-cells whereas association with Class II molecules will lead to generation of helper T-cells (Hahn and Kaufmann, 1981). Macrophages express a high amount of $\mathrm{Class}$ II antigen and this feature will allow preferential association of bacterial antigens with these molecules. Activation of macrophages by Class II restricted helper T-cells is triggered in intracellular bacterial infection and this event will lead to elimination of bacterial pathogens. On the other hand, Class I restricted cytotoxic T-cells will result in lysis of viral infected target cells inhibiting virus replication.

In view of these studies, it is possible that the identification of T-1ymphocytes of both the suppressor/cytotoxic $\mathrm{T}-\mathrm{cells}$ and helper/inducer T-cells, B-lymphocytes, HLA-DR ${ }^{+}$cells and macrophages in the prostate indicates their role in protecting this organ against infection. The observation that the prostatic biopsy, from the patient with chronic prostatitis, was infiltrated by a large number of T-cells, together with the finding that some of these were activated $\left(1 \mathrm{~L}-\mathrm{r}^{+}\right)$, indicates that the aetiology of some chronic prostatitis has an immunological component.

Activated T-cells, and specifically their products (1ymphokines), have been shown to facilitate the reactivity of macrophages not only by enhancing their capacity to present antigens (Scher et al., 1982) but also by augmenting their antimicrobial 
activity against a variety of pathogenic microorganisms (Nathan et a1., 1983). T-cell lymphokines can also enhance the oxygenindependent antimicrobial mechanisms of human mononuclear phagocytes (Murray et al., 1983).

We have shown in this study that Leu 7 , an antigen associated with $\mathrm{NK}-\mathrm{cells}$ ( $\mathrm{Abo}$ and $\mathrm{Balch}, 1981$ ), is also expressed on prostatic epithelium. This is in agreement with the recent observation of Wahab et al. (1985) who reported the reactivity of the anti-Leu 7 antibody with normal, benign and malignant prostatic tissues. They also suggested that Leu 7 antibody may be a useful diagnostic and prognostic marker of prostate cancer.

In contrast, the anti-Leu $11 \mathrm{~b}$ antibody which also defines the NK-cells, did not cross-react with the prostatic tissues. Leu $11 \mathrm{~b}^{+}$(Leu $7^{-}$) was detected within the epithelium and within the interstitial connective tissue stroma in BPH tissues. These results indicate that the Leu $11 \mathrm{~b}$ antibody, rather than the Leu 7 , is well suited to identifying $\mathrm{NK}-\mathrm{cells}$ in prostatic tissues.

It has been shown that the Leu $7^{+}$Leu $11^{-}$subset demonstrates low NK activity, whereas Leu $7^{-}$Leu $11^{+}$cells have a potent NK function (Abo et al., 1984). Although the clinical significance of $\mathrm{NK}-\mathrm{cells}$ is unknown, researchers postulate that they act as primary and early defence mechanisms against viral infections, through the cytotoxic and secretory activities and as a component of immune surveillance against tumour cell formation (Herberman and Ortaldo, 1981). 


\section{IV.G.2 Macrophages}

Little is known of macrophage maturation and function in vivo, because variations in macrophage morphology, enzyme content, and surface receptors hinder their recognition from other cell types. The recent development of monoclonal antibodies to human monocyte/macrophage surface antigens can now be used to identify normal resident mononuclear phagocytes and different stages of maturation and in several different tissues. Using these specific antibodies we have identified a resident population of mononuclear phagocytes and possibly dendritic cells within the tissues of the male genital tract: the testis, epididymis, vas deferens and the prostate. These cells were rarely seen in the seminal vesicle.

The presence in peripheral testis of relatively large numbers of cells reacting with anti-monocyte/macrophage monoclonal antibodies was unexpected in the light of previous observations using conventional histological techniques. While we cannot entirely exclude the possibility that certain of the monoclonals used cross-reacted with other cells, e.g. fibroblasts, we believe that the present studies indicate that there are significant numbers of monocytes/macrophages and possibly dendritic cells within the peripheral testis. In the first place these antisera failed to stain cultured human skin fibroblast or fibroblasts in prostatic tissue and skin. Three anti-macrophages/monocytes from independent sources (in addition to the antihuman leucocyte and the anti-HLA-DR antibodies) all gave the same staining patterns. This provides compelling evidence that the reaction is specific, since the three or the five reagents are unlikely to contain the same 
contaminating antibody. Furthermore, our observations are in agreement with previous histological studies in man (Holstein, 1978) and rat (Miller et al., 1984). In addition, the rat testis has recently been shown to produce large amounts of interleukin-1, which is known to be a macrophage derived protein (Khan et al., 1987).

The role of these macrophages in testicular tissues is not clear. In rats, testicular macrophages were observed in intimate association with Leydig cells, and portions of Leydig cell cytoplasm were endocytosed by macrophages (Miller et al., 1984). This prompted Bergh (1985) to suggest that these macrophages could be involved in the endocrine function of the testis. However, we believe that these macrophages may perform a more central immunological function. They may provide an ancillary back-up protection against the risk of escaped sperm antigens following compromise of the micro-anatomical blood-testis barrier. Alternatively, they may prevent the escape and dissemination of antigenic substances by destruction and removal by phagocytosis following alteration in the integrity of the blood-testis barrier. The demonstration of such cells in those segments of the genital tract, rete testis, epididymis and vas deferens, where luminal contents are absorbed or where sperm are stored, provides further support for their immunological role in the male reproductive tract. These cells could perform a direct phagocytic function against debris or sperm breakdown products and could also participate in the complex cellular interactions known to occur in both cellular and humoral immune responses (Friedmann et a1., 1981). In this context, it is interesting to note the expression of HLA-DR antigens on these macrophages or the presence of other $\mathrm{HLA}-\mathrm{DR}^{+}$cells, within 
the tissues of the male genital tract, which are thought to be essential for the successful presentation of antigen to T-cells (Kappler and Marrack, 1976). T-cells will recognize foreign antigens only when they are associated on the surface of a cell with a molecule encoded by the major histocompatibility complex (MHC). Thus cytotoxic T-cells usually see antigen in association with Class I MHC (molecules which are expressed on all cells) and helper T-cells usually see antigen in the context of MHC Class II molecules (Ia or Dr-like) which are expressed on a number of the cell varieties which comprise the immune system (Robertson, 1985). Whether or not these macrophages (Leu $3^{+}, 3.9^{+}$and $24^{+}$cells) are functioning as phagocytic or antigen presenting cells remains to be established. Nevertheless sperm phagocytosis was reported in the rete testis of the monkey and man (Holstein, 1978) and intraepithelial macrophages have been reported to occur throughout the length of the human epididymis (Wang and Holstein, 1983).

\section{IV.G.3 Patients with Testicular Obstruction}

Unilateral vasoligation and vasectomy have been shown to produce antisperm antibody in rats (Rumke and Titus, 1970). In man, Hendry et al. (1982) have reported on 32 subfertile humans with unilateral testicular obstruction, of whom $80 \%$ had antisperm antibodies and 50\% were severely oligozoospermic. The development of such antibodies in $60-80 \%$ of men undergoing bilateral vasectomy is well-established (Samuel and Rose, 1980). Meanwhile testicular biopsies in subfertile men with circulating antisperm antibodies 
have been reported as showing no evidence of allergic orchitis or immune depression of spermatogenesis (Rumke and Hellinger, 1959). Hargreave et al. (1982b) found no evidence of 1ymphocyte, macrophage or plasma cell infiltration in any testicular biopsies from 94 men with sperm density 1 ess than 10 million/ml and 9 men with obstructive azoospermia. Jarrow et al. (1985) have also found no evidence of inflammatory reactions in testicular biopsy specimens from 31 vasectomized men. Furthermore, Bigazzi et al. (1979) have reported that no human studies have revealed monocytic infiltration to be a characteristic of post-vasectomy testicular biopsy specimens. However, Hendry et al. (1979) have reported one patient with a high titre of antisperm antibody who had a focal round cell infiltrate around the seminiferous tubules.

Our study on testicular biopsies from subfertile men, using specific monoclonal antibodies with an indirect immunoperoxidase technique, has revealed the presence of lymphocytic infiltration, mainly of the T-1ymhocytes, in all the patients with testicular obstruction. The suppressor/cytotoxic T-1ymphocytes predominated in the biopsies from patients with obstructive azoospermia in whom no sperm antibodies were detected in either serum or semen. On the other hand, the helper/inducer T-cells predominated in biopsies from both patients with unilateral testicular obstruction and vas reversal patients, and this may explain in part the high titre of antisperm-antibodies noted in the serum and semen of these patients. It is of interest to note that epididymal biopsies from these groups of patients showed a more or less similar pattern of T-cell distribution. 
In the epididymal biopsies the pattern of distribution of the T-cell subsets was altered from that seen in normal epididymal tissues. There appeared to be a decrease in the suppressor/ cytotoxic T-cells within the epithelium and an increased infiltration by helper/inducer T-cells within the connective tissues of the lamina propria and interstitium. This was particularly marked in the patients with unilateral obstruction and vasectomy reversal. In addition, activated $\mathrm{T}$-cells were seen in these latter patients, indicating the presence of ongoing immune reactions. In such men, spermatozoa are produced continuously, although at a decreased rate, and accumulated within the blocked compartment of the excurrent genital ducts. These cells will disintegrate and breakdown in situ, resulting in increased resorption, phagocytosis and direct extravasation of sperm components. This, in turn, represents a powerful and continuous antigenic stimulus which may overwhelm and exhaust the capacity of the suppressor T-cell barrier. The resident suppressor T-cells, in the lining epithelia of the excurrent genital ducts, would under normal physiological conditions be exposed to a continuous but low dose of such sperm antigens.

As previously emphasized, it is now well established that $\mathrm{T}-\mathrm{cells}$ are involved in a complex series of interactions which regulate the immune response. Interaction between the Leu $2 \mathrm{a}^{+}$ cells and Leu $3 \mathrm{a}^{+}$cells is required to induce suppression of immunoglobulin production to antigen, pokeweed mitogen, or autologous mixed leucocyte reactions (Thomas et al., 1981). The Leu $3 a$ subset of cells on the other hand has been shown to proliferate in response to soluble antigens and to induce B-cells to secrete both immunoglobulin and precytotoxic cells to become 
cytotoxic (Reinherz et al., 1979). It has been also demonstrated that, with the appropriate target cells, a subset of Leu $3 a^{+}\left(\mathrm{T}_{4}^{+}\right)$ cells may be cytotoxic as well (Moretta et al., 1981). Nevertheless, the demonstration that Leu $3 \mathrm{a}^{+}\left(\mathrm{T}_{4}^{+}\right)$cytotoxic lymphocytes are specific for Class II molecules (DR-antigens) provides a mechanism for tissue damage. Such cytotoxic cells could conceivably damage a variety of cells bearing Class II antigens including endothelial cells. In addition, loss or decrease in the Leu $2 \mathrm{a}^{+}\left(\mathrm{T}^{+}\right)$cells may result in unopposed inducer functions and the development of unregulated cytotoxic Leu $3 \mathrm{a}^{+}\left(\mathrm{T}_{4}^{+}\right)$cells.

A delicate balance between effector and regulatory cells normally exists and is required for immune homeostasis. Aberrations in immunoregulatory T-cell subsets have been shown to exist in many autoimmune diseases, e.g. rheumatoid arthritis, systemic lupus erythematosus and graft versus host disease. These diseases are generally characterised by an increase in the ratio of the helper/inducer to the suppressor/cytotoxic T-cells. These changes in the T-lymphocyte subsets are associated with reduced or impaired suppressor activity and may be accompanied by the development of autoantibodies and/or cytotoxic cell activity (Schlossman and Reinherz, 1984).

In view of these studies, it is most likely that the predominant suppressor/cytotoxic T-cells in the epithelial lining of the normal male genital tract function as a major immunological barrier which may normally prevent the development of autohumoral or cellular immune responses to sperm antigens. Obstruction to the outflow of spermatozoa resulted in: increased infiltration of testicular and epididymal biopsies, from patients with unilateral 
obstruction and vasectomy reversal, by Leu $3 \mathrm{a}^{+}$cells; 1oss of Leu $2 \mathrm{a}^{+}$cells and imbalance of the suppressor/inducer cell ratios in epididymal biopsies. The finding of increased infiltration of testicular and epididymal biopsies with T-cells, together with the observation that some of these are activated, or $1 \mathrm{~L}-2 \mathrm{r}^{+}$, suggests the presence of antisperm cell-mediated reactions. It also elucidates the mechanism of sperm antibody production. The observation that the unilaterally obstructed patients resemble vasectomised men as regards the pattern and type of cell infiltrate and the presence of sperm antibodies in their serum and seminal plasma may indicate the clinical significance of unilateral testicular obstruction in subfertile males. This has been recently reported by Hendry (1986). 
CHAPTER V

LYMPHOCYTE SUBSETS IN UROTHELIUM 


\section{V.A INTRODUCTION}

The mucosal lining of the urinary tract is being constantly challenged by a variety of biological and chemical agents which include microorganisms, and toxic or carcinogenic substances. The development of mucosal as well as systemic immune responses to microbial and other antigens is controlled and regulated by specific immunoregulatory mechanisms. T-lymphocytes and macrophages are known to play a major role in such immune-regulation.

Having defined the 1ymphocyte subsets and macrophages in the tissues of the reproductive tract, we find that it is worthwhile to extend these studies to the urinary tract, particularly the urothelium. This section reports the results in normal human urothelium and the preliminary studies on patients with bladder tumour (carcinoma in situ).

\section{V.B NORMAL UROTHELIUM}

Histological examination of sections stained with haematoxylin and eosin showed normal urothelium and subjacent lamina propria. All the specimens showed the same immunostaining profile.

\section{V.B.1 T-Lymphocytes}

T-1ymphocytes stained with the pan T-cell monoclonal antibody (T11) were demonstrated within the mucosal lining of both the ureter and the urinary bladder. They showed a uniform 
distribution in the urothelium around the entire circumference of the ureter, while in the bladder cellular distribution within the mucosa was patchy. In both the ureter and the urinary bladder, the T-1ymphocytes were situated mainly within the epithelium and the lamina propria with the greater proportion of them being present within the epithelium. T-lymphocytes were situated alongside the epithelial cells at various levels above the basement membrane but tended to be concentrated towards the basal layer. In the lamina propria the T-lymphocyte cells were found towards the upper part of the subepithelial connective tissue layers also in close proximity to the other surface of the basement membrane.

The majority of these mucosal T-1ymphocytes were identified as $\mathrm{T}^{+}$, i.e. of the suppressor/cytotoxic phenotype (Figure V.1). In the ureter, $84.1 \%$ of the intraepithelial T-1ymphocytes and $61.3 \%$ of the lamina propria T-1ymphocytes exhibited the suppressor/ cytotoxic phenotype. In the urinary bladder the distribution of T-1ymphocytes was similar; $87.7 \%$ of the intraepithelial T-cells and $60.7 \%$ of the lamina propria T-cells expressed the suppressor/ cytotoxic phenotype (Tables V.1 and V.2).

Some $\mathrm{T}^{+}$(helper/inducer T-1ymphocytes) were also detected both within the epithelium and the lamina propria of the mucosal lining of the ureter and the urinary bladder and were located mainly in the lamina propria. They were fewer in number in comparison to the $\mathrm{T}^{+}$cells. The average ratios of the suppressor/cytotoxic $\mathrm{T}$-cells to the helper/inducer T-cells $\left(\mathrm{T}^{+} / \mathrm{T}^{+}{ }^{+}\right.$) were 10.4 and 7.4 in the urothelium of the ureter and the urinary bladder respectively. None of the T-lymphocytes showed a detectable reaction with the 

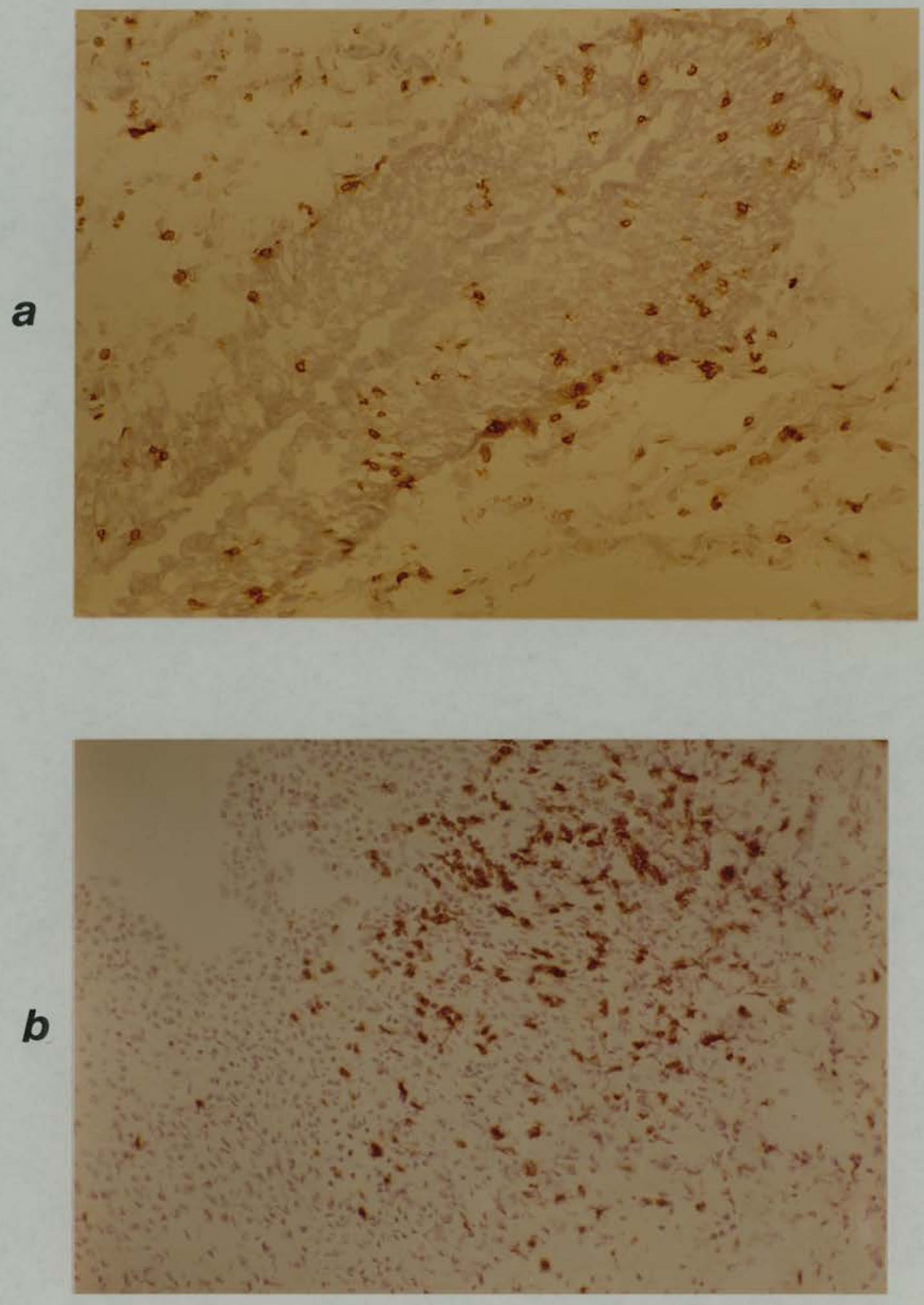

Figure V.1: $\mathrm{T}^{+}$cells (suppressor/cytotoxic T-1ymphocytes) within normal urothelium: a) ureter; b) urinary bladder. (Cryostat sections, immunoperoxidase, x 160 and x 80) 


\section{TABLE V.1:}

T-LYMPHOCYTE SUBSETS WITHIN THE EPITHELIUM AND LAMINA PROPRIA OF NORMAL HUMAN URETER

\begin{tabular}{|c|c|c|c|c|}
\hline $\begin{array}{l}\text { Live } \\
\text { Donor }\end{array}$ & $\begin{array}{l}\mathrm{T}_{11}^{+} \text {cells } \\
\text { (A11 T-1ympho- } \\
\text { cytes) }\end{array}$ & $\begin{array}{c}\mathrm{T}^{+} \text {cells } \\
\text { ( } \mathrm{T} \text { suppressor/ } \\
\text { cytotoxic) }\end{array}$ & $\begin{array}{l}\mathrm{T}^{+} \text {cells } \\
\text { ( } \mathrm{T} \text { helper/ } \\
\text { inducer) }\end{array}$ & $\mathrm{T}^{+} / \mathrm{T}^{+}+$ \\
\hline & \multicolumn{4}{|c|}{ 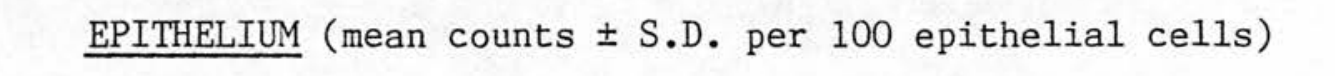 } \\
\hline A & 14.30 & 10.74 & 1.20 & 8.95 \\
\hline B & 9.43 & 8.43 & 1.38 & 6.10 \\
\hline $\mathrm{C}$ & 11.24 & 9.57 & 0.95 & 10.07 \\
\hline D & 12.12 & 11.34 & 0.62 & 18.29 \\
\hline $\mathrm{E}$ & 10.79 & 8.03 & 0.58 & 13.84 \\
\hline All donors & $11.57 \pm 1.80$ & $9.62 \pm 1.42$ & $0.94 \pm 0.35$ & $11.45 \pm 4.72$ \\
\hline
\end{tabular}

LAMINA PROPRIA (mean counts \pm S.D. per $0.8 \mathrm{~mm}^{2}$ tissue $\mathrm{x} 160$ )

$\begin{array}{lrrrr}\text { A } & 12.1 \pm 2.7 & 6.6 \pm 1.5 & 4.8 \pm 2.0 & 1.37 \\ \text { B } & 12.8 \pm 2.3 & 7.9 \pm 1.2 & 5.9 \pm 1.1 & 1.33 \\ \text { C } & 16.7 \pm 2.9 & 11.0 \pm 1.8 & 7.0 \pm 1.2 & 1.57 \\ \text { D } & 18.1 \pm 2.3 & 11.1 \pm 1.9 & 6.9 \pm 1.4 & 1.60 \\ \text { E } & 15.5 \pm 3.4 & 9.5 \pm 1.6 & 5.0 \pm 1.3 & 1.90\end{array}$

$\begin{array}{lllll}\text { All donors } 15.04 \pm 2.54 & 9.22 \pm 1.96 & 5.92 \pm 1.02 & 1.55 \pm 0.22\end{array}$

(See Appendix C) 


\section{TABLE V.2:}

T-LYMPHOCYTE SUBSETS WITHIN THE EPITHELIUM AND LAMINA PROPRIA OF NORMAL HUMAN BLADDER

\begin{tabular}{|c|c|c|c|c|}
\hline $\begin{array}{l}\text { Cadaver } \\
\text { Donor }\end{array}$ & $\begin{array}{c}\mathrm{T}_{11}^{+} \text {cells } \\
\text { (A11 T-lympho- } \\
\text { cytes) }\end{array}$ & $\begin{array}{l}\mathrm{T}^{+} \text {cells } \\
\text { ( } \mathrm{T} \text { suppressor/ } \\
\text { cytotoxic) }\end{array}$ & $\begin{array}{l}\mathrm{T}^{+} \text {cells } \\
\text { ( } \mathrm{T} \text { helper/ } \\
\text { inducer) }\end{array}$ & $\mathrm{T}^{+} / \mathrm{T}^{+}+$ \\
\hline
\end{tabular}

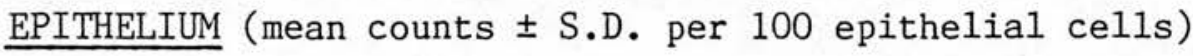

$\begin{array}{rrrrr}4 & 7.87 & 6.94 & 0.73 & 9.50 \\ 5 & 10.84 & 7.10 & 0.92 & 7.71 \\ 6 & 9.67 & 7.88 & 1.16 & 6.79 \\ 7 & 9.39 & 8.23 & 1.15 & 9.46 \\ 8 & 9.14 & 6.79 & 1.05 & 6.46\end{array}$

A11 donors

$9.38 \pm 1.06$

$7.38 \pm 0.63$

$1.002 \pm 0.18$

$7.98 \pm 1.44$

LAMINA PROPRIA (mean counts \pm S.D. per $0.8 \mathrm{~mm}^{2}$ tissue $\mathrm{x} 160$ )

$\begin{array}{lrrrr}4 & 9.3 \pm 1.3 & 5.3 \pm 1.3 & 3.7 \pm 0.7 & 1.43 \\ 5 & 13.8 \pm 2.3 & 8.4 \pm 1.4 & 5.6 \pm 1.8 & 1.50 \\ 6 & 11.8 \pm 3.3 & 7.5 \pm 1.7 & 5.5 \pm 1.4 & 1.36 \\ 7 & 10.2 \pm 1.5 & 5.0 \pm 0.9 & 3.0 \pm 0.8 & 1.66 \\ 8 & 11.0 \pm 1.8 & 7.6 \pm 2.1 & 5.1 \pm 1.5 & 1.49\end{array}$

$\begin{array}{lllll}\text { Al1 donors } & 11.22 \pm 1.71 & 6.76 \pm 1.51 & 5.67 \pm 1.71 & 1.48 \pm 0.11\end{array}$

(See Appendix C) 
anti-interleukin-2 receptor monoclonal antibody which detects activated T-cells.

\section{V.B.2 B-Lymphocytes}

Cells reacting with the B1 antibody which detects B-1ymphocytes were seen as small lymphoid aggregates in the outer part of the serosa of the ureter. No $\mathrm{BI}^{+}$cells were detected in the mucosal lining of the ureter and the urinary bladder.

\section{V.B.3 Monocytes and Macrophages}

Cells stained by the anti-Leu M3 monoclonal antibody, which reacts with cell-surface antigens of monocytes/macrophages, were few in number and were detected mainly in the lamina propria. Occasional Leu $\mathrm{M}^{+}$cells were also seen within the urothelium.

\section{V.B.4 Anti-HLA-DR Reactive Cells}

The majority of mucosal cells were HLA-DR negative but scattered HLA-DR positive cells were observed within the epithelium and within the lamina propria. Within the epithelium, the distribution of $\mathrm{HLA}_{-\mathrm{DR}^{+}}$cells was similar to that of the T-1ymphocytes. On the other hand, the HLA-DR ${ }^{+}$cells detected in the lamina propria had a dendritic morphology (Figure V.2). However, $\mathrm{T}^{+}$cells identified in the lamina propria were very few in numbers compared with the HLA-DR ${ }^{+}$cells. 


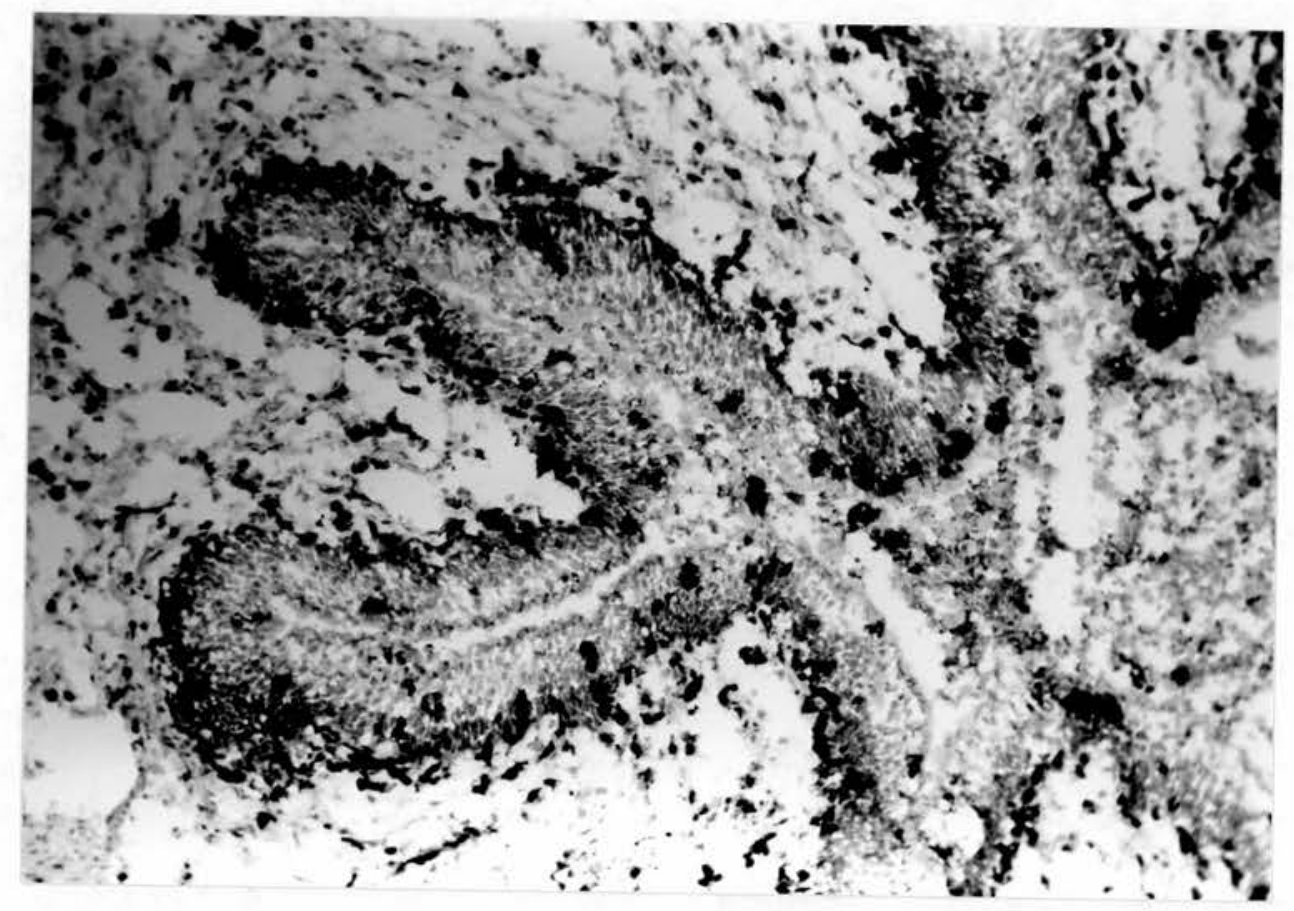

Figure V.2: $\quad H L A-D R^{+}$cells within the epithelium and lamina propria of normal human ureter.

(Cryostat sections, immunoperoxidase, x 80) 


\section{V.C BLADDER BIOPSIES FROM PATIENTS WITH CARCINOMA IN SITU}

V.C.1 T-Lymphocytes

The three biopsies examined showed massive infiltration with T-lymphocytes throughout the yrothelium and the lamina propria. In contrast to normal tissues, the T-lymphocytes tended to be concentrated in the lamina propria. In addition the majority of these T-cells stained with the T4 antibody which defines the helper/inducer T-cells (Figure V.3a). The T8 antibody stained a minority of T-cells. The expression of IL2-receptors was demonstrated on a substantial proportion of cells.

\section{V.C.2 B-Lymphocytes}

In the lamina propria, B-lymphocyte aggregates and/or follicles were detected (Figure V.3b). These B-cell aggregates (follicles) were often surrounded by a halo of $\mathrm{T}^{+}$(helper/inducer) T-cells.

\section{V.C.3 Monocytes/Macrophages}

Cells stained by Leu M3 antibody were detected and appeared more numerous compared with the normal tissue. 

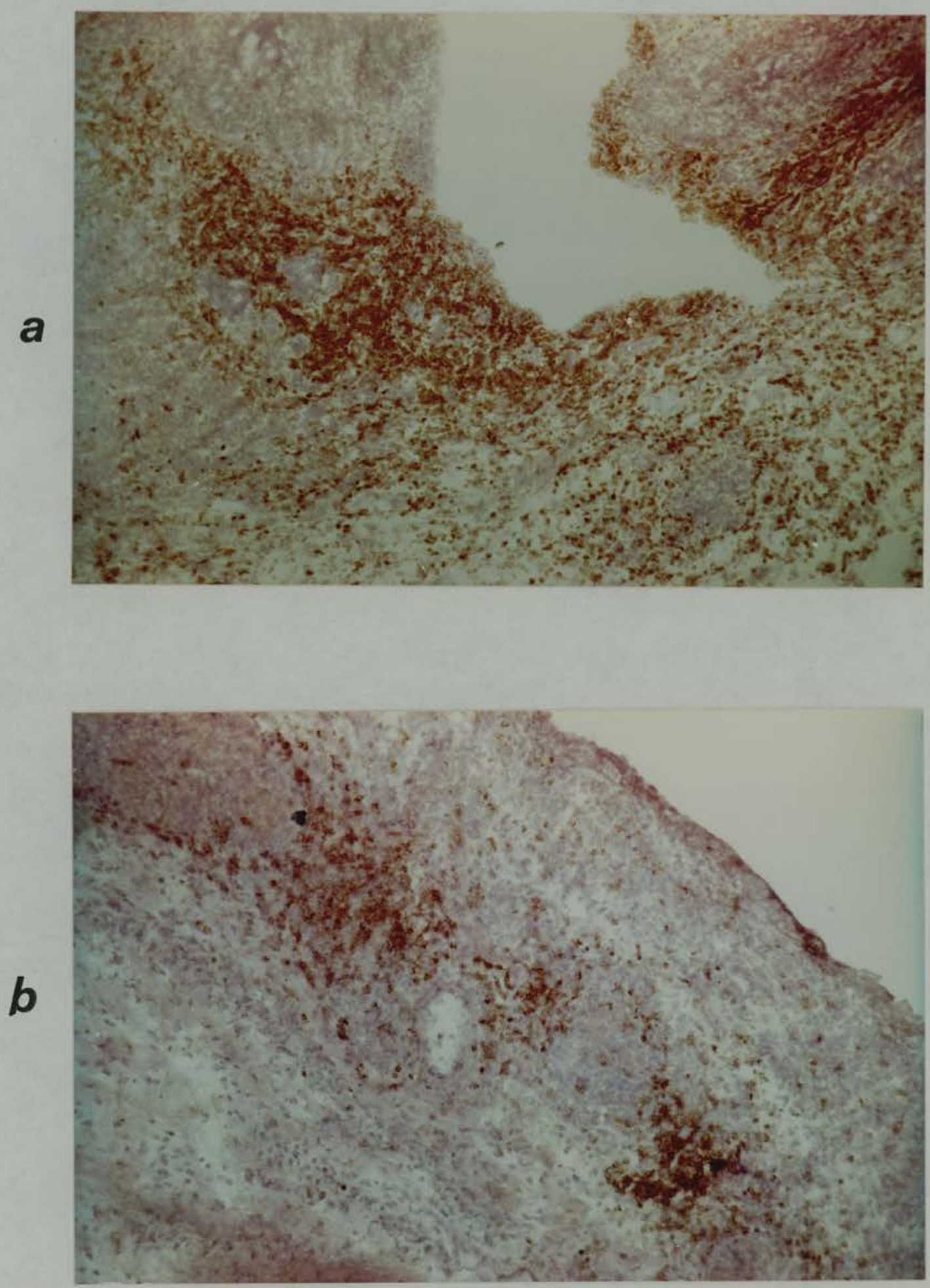

Figure V.3: Immunoperoxidase staining of cryostat sections of a bladder biopsy obtained from a patient with carcinoma in situ stained with a) T4 and b) B1 monoclonal antibodies.

Note the infiltration of the urothelium with $\mathrm{T}_{4}^{+}$cells and the presence of $\mathrm{Bl}^{+}$follicles in the lamina propria ( $\mathrm{x} 80$ ). 


\section{V.C.4 Anti-HLA-DR Reactive Cells}

Unlike the normal urothelium which was HLA-DR-negative, the urothelia, in these three biopsies, displayed strong staining with the anti-HLA-DR antibody. Furthermore, all the 1ymphocytes and macrophages were HLA-DR-positive.

\section{V.D DISCUSSION}

Using monoclonal antibodies in an indirect immunoperoxidase technique, lymphocyte subsets and macrophages in normal ureteric and bladder urothelium were identified. Lymphocytes were located within the epithelium as well as in the subepithelial connective tissues and were almost always T-cells with a preponderance of the suppressor/cytotoxic phenotype. The lymphocytes were concentrated within the epithelium, whereas $81.3 \%$ of the intraepithelial T-1ymphocytes were of the suppressor/cytotoxic phenotype.

The pattern of 1ymphocyte distribution observed in normal human urothelium is very similar to that noted in the lining epithelia of normal human rete testis, epididymis, vas deferens, prostate and seminal vesicles where it was shown that approximately $70 \%$ of the T-1ymphocytes found within the mucosa of the male genital tract were of the suppressor/cytotoxic phenotype. These results are in agreement with the recent observations of Gardiner et al. (1986) who reported the presence of T-1ymphocytes mainly of the suppressor/cytotoxic phenotype within normal human bladder mucosa. In addition Selby and his colleagues (1981) have shown that $79 \%$ of 
the intraepithelial T-lymphocytes in the intestinal mucosa were also of the suppressor/cytotoxic phenotype. These findings suggest a probable common pattern of deployment of immune competent cells within mucosal surfaces and perhaps a shared mucosal immunity system. The urothelium has a wide surface area on which there is an interface between the environment and the tissues and, although contamination with microorganisms is frequent, frank infections are rare. Similarly, urine may contain numerous carcinogenic agents and yet urothelial malignancy is limited.

The presence of T-1ymphocyte subsets within the urothelium and within the lamina propria may imply that immune related mechanisms may be directly involved in protection of the ureter and the bladder mucosa. The obvious preponderance of T-cells, macrophages and cells expressing HLA-DR antigens, combined with the paucity of B-cells, suggests that cell-mediated immunity is more important than humoral immunity in effecting a local mucosal resistance. However it is not clear whether these demonstrable $\mathrm{T}^{+}$ cells are functioning in a suppressor or in a cytotoxic role as both 1ymphocyte subsets are detected by this reagent. If indeed these lymphocytes were predominantly of the suppressor phenotype, it might be argued that their functional presence within the urothelium was to protect the epithelial microenvironment from immune mediated damage. If however the $\mathrm{T}^{+}$cells were mainly of the cytotoxic phenotype, then they would be of value both in resistance against certain infections and in tumour surveillance. Cytotoxic T-cell mediated immune reactions could lead to the removal of damaged cells as well as urothelial cells with adherent bacteria, infected cells and cells damaged by environmental chemicals. It has been 
suggested that, when an agent destroys a group of intestinal mucosal epithelial cells, local release of cytotoxic cells would provide a first line of cell-mediated defence of the de-epithelialised area with macrophages further supplementing this initial action (Mowat and Ferguson, 1981).

The presence of macrophages (Leu $\mathrm{M}^{+}$cells) within the mucosa of the ureter and the urinary bladder suggests that they also have a role in local mucosal immunity. They could perform a direct cytotoxic function and/or phagocytic function against the invading microorganisms or against aberrant cells and could also participate in the complex cellular interactions known to occur in both cellular and humoral immune responses (Friedman et al., 1981). In this context it is interesting to note the presence within the urothelium of cells possessing cell surface Class II ( $\mathrm{Ia} / \mathrm{Dr}-1$ ike) antigens which are thought to be essential for the successful presentation of antigen to T-cells (Kappler and Marrack, 1976; Stingl et al., 1978). This finding further suggests that cell-mediated immunity is of more importance than humoral immunity in the mucosa.

of particular interest were the changes in lymphocyte distribution noted in the few biopsies examined from patients with carcinoma in situ. The most notable changes were: increased infiltration with $\mathrm{T}$-cells of the helper/inducer phenotype $\left(\mathrm{T}^{+}\right)$and macrophages (Leu $\mathrm{M}^{+}$); the demonstration of $\mathrm{B}$-1ymphocytes $\left(\mathrm{BI}^{+}\right.$) aggregates in the lamina propria; and the expression of the HLA-DR antigens (Class II molecules) on the urothelial cells as well as the infiltrating lymphoid cells. These changes indicate a host immune response to the tumour though the relevance of such to tumour growth remains to be established. While there are many ways such a 
response could retard tumour growth including both cells of the immune system and antibodies, most tumours eventually go on or progress to kill the patient.

In conclusion, using specific monoclonal antibodies, T-1ymphocyte subsets and macrophages were identified in normal urothelium and shown to have a specific pattern of distribution similar to that noted in the lining epithelium of the genital tract. The existence of these cell populations may contribute to the health and protection of the urothelium, particularly in resistance to infection and tumour surveillance. These cell populations should also be considered in relation to the understanding of mucosal immunity in general. Profound changes in the incidence and distribution of these cells were noticed in bladder biopsies from patients with carcinoma in situ. 
CHAPTER VI

IDENTIFYING LEUCOCYTES AND LEUCOCYTE SUBPOPULATIONS IN SEMEN USING MONOCLONAL ANTIBODY PROBES 


\section{VI.A INTRODUCTION}

This chapter reports the results of immunocytochemical detection of leucocyte subpopulations, combined with the microbiological screening, in the ejaculate of 81 men (see materials and methods, section II.B.4) and discusses the findings.

\section{VI.B IMMUNOPEROXIDASE STAINING OF CYTOCENTRIFUGED SMEARS OF SEMEN}

The monoclonal antibodies used to study leucocytes and leucocyte subpopulations in the ejaculate of 69 subfertile men and 12 infertile men included: the antihuman leucocytes which defines all leucocytes; the anti-Leu 4 which defines all T-cells; the anti-Leu 3 a which defines the T-helper/inducer cells; the anti-Leu 2a which defines the T-suppressor/cytotoxic cells and the anti-Leu 12 which defines B-lymphocytes (see Table II.2 in materials and methods).

The staining pattern seen in semen smears was similar to that obtained in peripheral blood leucocyte smears, the positive controls. The positively stained cells were easily identified by their dark brown cell membrane and/or cytoplasmic staining. The majority of these stained cells appeared from their morphological appearance to be PMN leucocytes. Cells actively involved in phagocytosis showed specific staining. The distribution of the positively labelled cells was nearly uniform throughout the smears and in any particular specimen the count from one field to another 
was consistent. A few leucocytes showed yellowish brown peroxidase positive granules in their cytoplasm. Negative control smears showed no staining.

\section{VI.C LEUCOCYTES IN THE SEMEN}

\section{VI.C.1 Fertile Men}

A11 of the specimens from the 12 fertile men showed cells reacting with the pan anti-leucocyte monoclonal antibody with a mean count of $44.9 \pm 25.8$ leucocytes per HPF (x 320) (Table VI.1a). Most of these cells appeared from their morphological appearances to be polymorphonuclear leucocytes (Figure VI.1a). Cells reacting with the antilymphocyte reagents were rarely detected.

\section{VI.C.2 Patients with an Infertile Marriage}

Leucocytes were detected in $90 \%(63 / 67)$ of the specimens from the patients, with a mean count of $14.6 \pm 17.1$ leucocytes per HPF (x 320). T-1ymphocytes (Leu $4^{+}$cells) were demonstrated in 13 subfertile patients, with a mean count of $4.5 \pm 3.3$. The mean count of all leucocytes in these 13 patients was $15.6 \pm 12.6$ leucocytes/HPF T-1ymphocytes/HPF (x 320) (Table VI.1b). The T-lymphocytes were mainly of the suppressor/cytotoxic phenotype (Leu $2 \mathrm{a}^{+}$) (Figure VI.1b). Although Leu $3 \mathrm{a}^{+}$cells (T-helper/inducer cells) were seen occasionally, no Leu $12^{+}$cells (B-1ymphocytes) were detected in any of the specimens. The number of PMN leucocytes was 
TABLE VI.1a:

LEUCOCYTES IN SEMEN DETECTED BY ANTIHUMAN LEUCOCYTE (HLe-1) MONOCLONAL ANTIBODY

Number of leucocytes

per HPF (x 320)

Number of patients

Number of fertile

men (controls)

$\begin{array}{lrr}0 & 7 & 0 \\ 1-10 & 35 & 0 \\ 11-20 & 15 & 2 \\ 20 & 13 & 10\end{array}$

TABLE VI.1b:

T-LYMPHOCYTES IN SEMEN DETECTED BY ANTI-LEU 4 MONOCLONAL ANTIBODY (A PAN T-CELL MARKER)

$\begin{array}{cc}\begin{array}{c}\text { Number of T-1ymphocytes } \\ \text { per HPF (x 320) }\end{array} & \text { Number of fertile } \\ \text { men (controls) }\end{array}$

0

50

11

$1-10$

12

1

$11-20$

1

0 

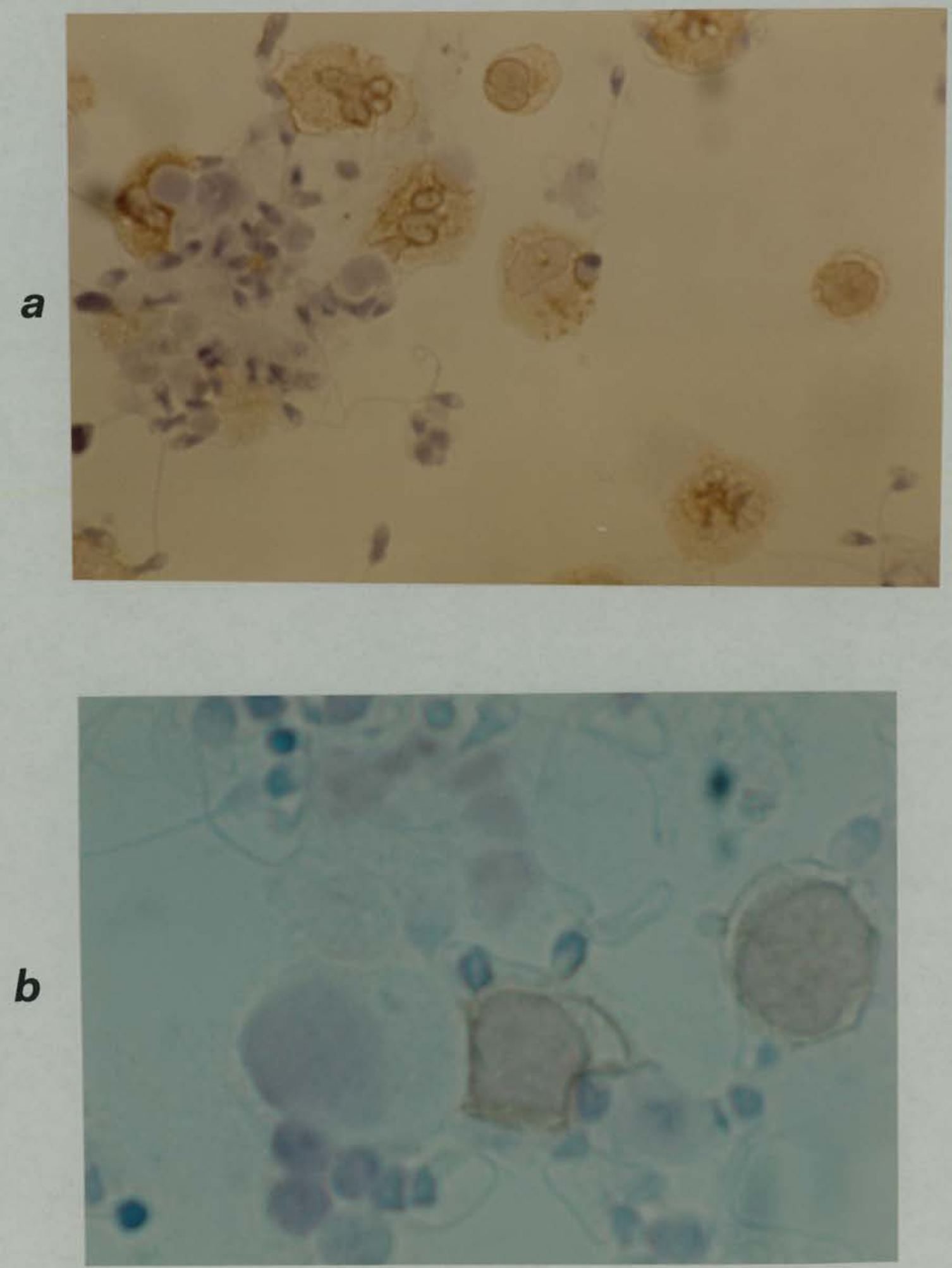

Figure VI.1: Immunoperoxidase staining of cytocentrifuge smears of semen stained with:

a) antihuman leucocyte antibody for all leucocytes

b) Leu $2 \mathrm{a}$ antibody for suppressor/cytotoxic cells

(a x 320; b x 640) 
calculated by subtracting the total lymphocyte count from the total leucocyte count. Wilcoxon rank sum tests showed that the total leucocyte count and the PMN leucocytes were significantly higher in fertile men $(P<0.001$ in both). However, there were no significant differences in the counts of T-1ymphocytes or their subsets. This possibly is a reflection of the low incidence of T-1ymphocytes in the ejaculate examined.

\section{VI.D MICROBIOLOGICAL INVESTIGATIONS}

Sixty-five patients and eight fertile men were tested for the following microorganisms: aerobes, anaerobes, Ureaplasma urealyticum and Chlamydia trachomatis. Fifty-seven of the 65 patients and 7 of the 8 fertile men were found to have positive bacterial cultures for one or more of these microorganisms (Table VI.2). Eight patients and one fertile man had completely negative results. There were no positive cultures for Chlamydia.

Aerobic organisms were isolated either alone or in association with anaerobes of Ureaplasma urealyticum from 55 patients and seven fertile men. Anaerobes were isolated from 43 patients and from all of the fertile men with positive cultures. Nine patients and one fertile man had positive cultures for Ureaplasma urealyticum.

There were no significant differences in bacterial counts among individual organisms or groups except for Proteus, which was significantly more common in the fertile men $(2 / 8)$ compared with the patients $(2 / 65)$. The most frequent organism in both patients and 
No. of patients with corresponding number

No. of bacterial

of species (and total number of isolates)

species isolated per patient

Infertile patients

Controls

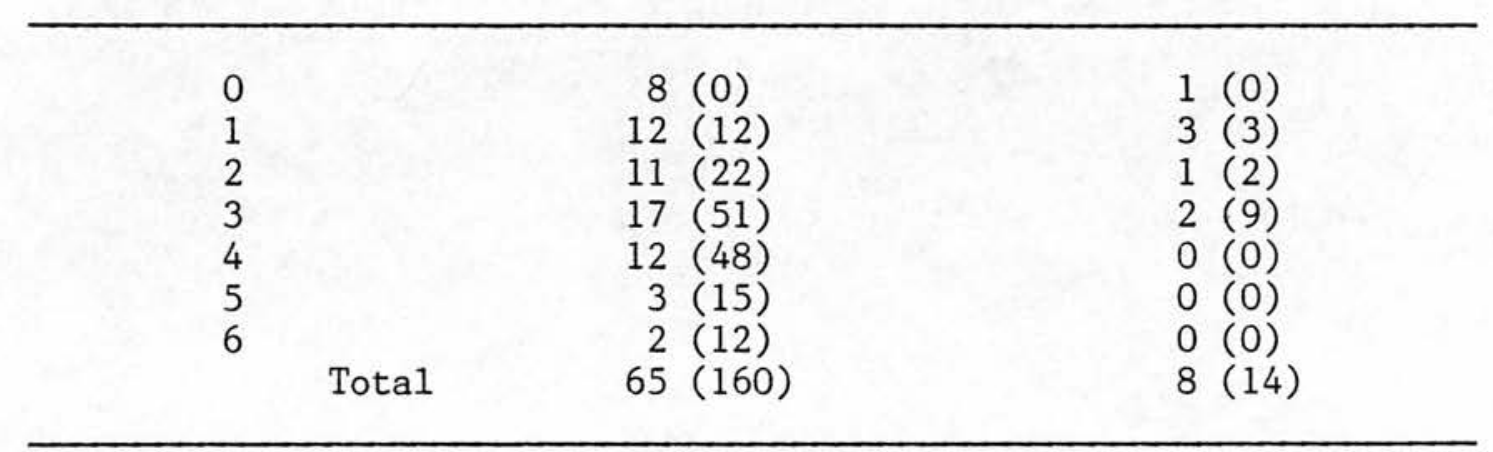

* Excluding Ureaplasma urealyticum

Notes:

The mean number of organisms per control sample $=1.75(14 / 8)$

The mean number of organisms per infertile sample $=2.46(160 / 65)$

None of the controls has 3 bacterial species present per sample

TABLE VI.3: ISOLATION RATE OF BACTERIA FROM 73 SEMEN SAMPLES

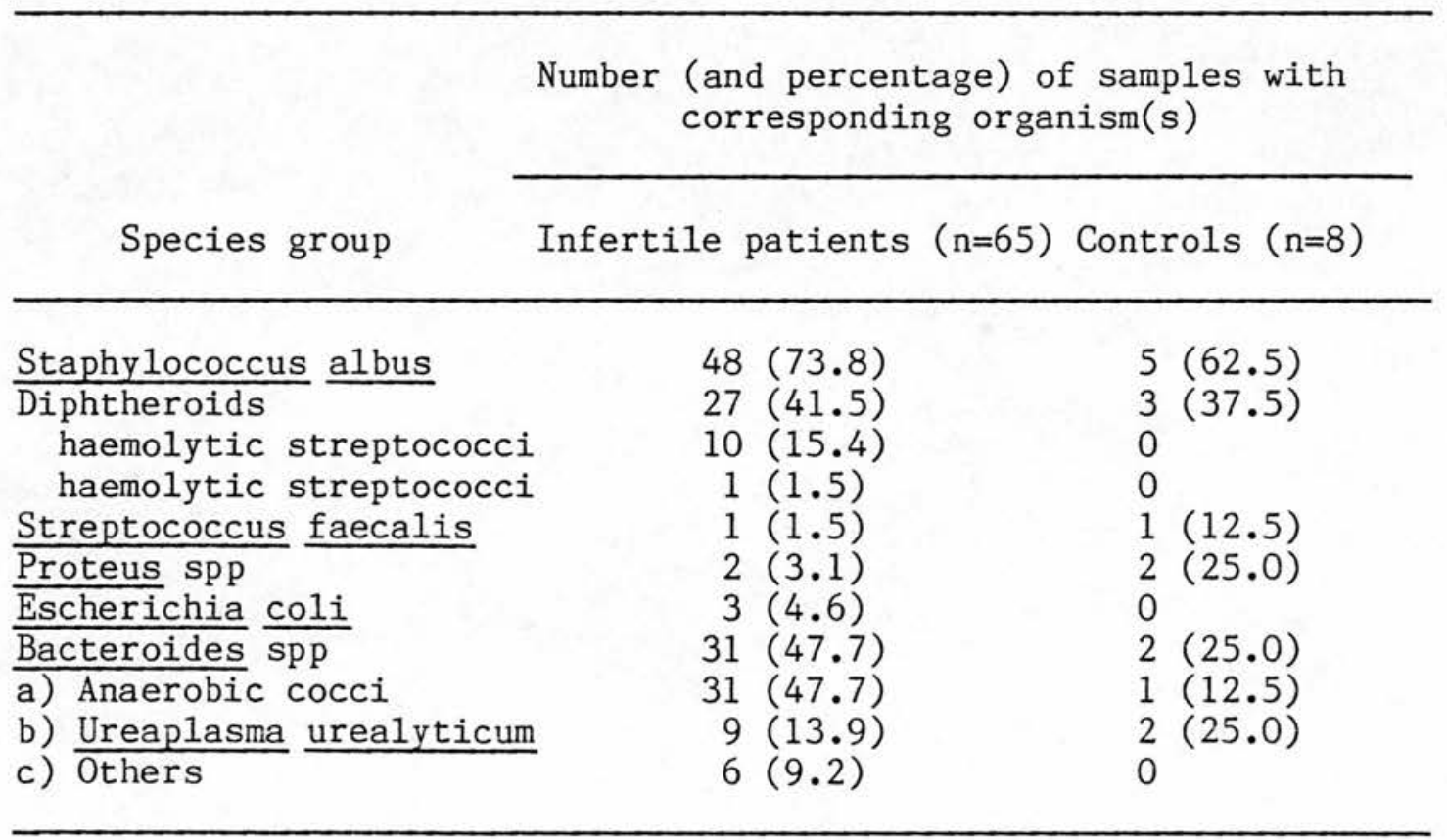

a) Bacteroides and/or anaerobic cocci were isolated from 43 (66.2\%) samples from infertile patients and from 3 (37.5\%) samples from controls.

b) Ureaplasma isolation rate based on 70 valid cultures.

c) Includes: Fusobacterium nucleatum 1; Eubacterium Lentium 2;

Proprionibacterium acnes 1; Gardnere1la vaginalis 1; and

Lactobacillus sp. 1 . 
TABLE VI.4:

QUANTITATIVE BACTERIOLOGY OF MOST FREQUENTLY ISOLATED ORGANISMS

\begin{tabular}{|c|c|c|}
\hline Group/species & $\begin{array}{l}\text { No. of } \\
\text { isolates }\end{array}$ & $\begin{array}{l}\text { Range (and mean number) of } \\
\text { colony forming units* } \\
\text { isolated }\end{array}$ \\
\hline Anaerobic cocci & 32 & \\
\hline Peptococcus magnus & 13 & $1.0 \times 10^{3}-5 \times 10^{5}\left(9.7 \times 10^{4}\right)$ \\
\hline Peptococcus asaccharolyticus & 8 & $1.0 z 10^{3}-3 \times 10^{5}\left(4.2 \times 10^{4}\right)$ \\
\hline Peptococcus prevottii & 6 & $2.0 \times 10^{3}-1.5 \times 10^{4}\left(7.4 \times 10^{3}\right)$ \\
\hline Peptococcus anaerobius & 1 & $2.5 \times 10^{4}$ \\
\hline Peptostreptococcus anaerobius & 3 & $5.0 \times 10^{3}-1.0 \times 10^{6}\left(3.4 \times 10^{5}\right)$ \\
\hline Unidentified & 1 & $2.0 \times 10^{3}$ \\
\hline Bacteroides spp. & 32 & \\
\hline Bacteroides asaccharolyticus & 9 & $1.0 \times 10^{3}-1.0 \times 10^{6}\left(3.2 \times 10^{5}\right)$ \\
\hline$\underline{\text { Bacteroides bivius }}$ & 4 & $5.0 \times 10^{3}-1.0 \times 10^{5}\left(5.5 \times 10^{4}\right)$ \\
\hline Bacteroides melaninogenicus & 2 & $3.0 \times 10^{3}-4.0 \times 10^{5}\left(2.0 \times 10^{5}\right)$ \\
\hline Bacteroides oralis & 2 & $1.5 \times 10^{3}-5.0 \times 10^{3}\left(3.2 \times 10^{3}\right)$ \\
\hline Bacteroides corporis & 1 & $5.5 \times 10^{3}$ \\
\hline Bacteroides disiens & 1 & $1.0 \times 10^{6}$ \\
\hline Bacteroides intermedius & 1 & $5.0 \times 10^{4}$ \\
\hline Unidentified & 12 & $3.0 \times 10^{2}-4.5 \times 10^{6}\left(3.7 \times 10^{5}\right)$ \\
\hline Staphylococcus albus & 50 & $1.5 \times 10^{2}-1.5 \times 10^{6}\left(1.1 \times 10^{5}\right)$ \\
\hline Diptheroids & 28 & $5.0 \times 10^{3}-4.0 \times 10^{6}\left(4.9 \times 10^{5}\right)$ \\
\hline haemolytic streptococci & 10 & $2.0 \times 10^{2}-2.0 \times 10^{5}\left(3.4 \times 10^{4}\right)$ \\
\hline Ureaplasma urealyticum & 10 & $1.0 \times 10^{1}-1.0 \times 10^{4}\left(2.1 \times 10^{3}\right.$ \\
\hline
\end{tabular}

* Colour changing units in case of Ureaplasma urealyticum 
fertile men was Staphylococcus albus (Tables VI.3 and 4) with a mean (range) count of colony forming units isolated of $1.1 \times 10^{5}$ $\left(1.5 \times 10^{2}-1.5 \times 10^{6}\right)$. Correlating the bacterial count of every individual organism detected with sperm density and motility, the results were not significant except for Bacteroides asaccharolyticus, which showed a negative correlation with density and motility $(P<0.05)$.

\section{VI.E RELATIONSHIP OF LEUCOCYTES TO BACTERIAL COUNTS}

Each individual bacterial count, ureaplasma and bacterial groups were correlated with the leucocyte and leucocyte subpopulation counts. The results were not statistically significant (Tables VI.5, 6 and 7) except that B-streptococci showed a significant positive correlation with T-lymphocytes $\left(\begin{array}{ll}P & 0.05\end{array}\right)$.

\section{VI.F RELATIONSHIP OF LEUCOCYTES TO ANTISPERM ANTIBODIES}

Serum TAT results were available for 50 patients and MAR IgG for 19. Of the correlations with leucocyte counts, on 1y that between MAR IgG and PMN leucocytes was significant (positive P 0.05). However, there was no significant correlation with serum TAT (Table VI.8). 
TABLE VI.5: RELATIONSHIP BETWEEN NUMBER OF BACTERIAL SPECIES PER SEMEN SAMPLE AND LEUCOCYTE COUNT

\begin{tabular}{crrrr}
$\begin{array}{c}\text { No. of bacterial } \\
\text { species isolated } \\
\text { per patient }\end{array}$ & 1 & $\begin{array}{c}\text { Number of patients with } \\
\text { leucocyte counts of : } \\
1-20\end{array}$ & 20 & Total \\
\hline 0 & 1 & 3 & 2 & 6 \\
1 & 1 & 8 & 3 & 12 \\
2 & 4 & 6 & 1 & 11 \\
3 & 5 & 10 & 2 & 17 \\
4 & 2 & 8 & 2 & 12 \\
5 & 1 & 2 & 0 & 3 \\
6 & 0 & 1 & 1 & 2 \\
Total & 14 & 38 & 11 & 63 \\
\hline
\end{tabular}

Kendall rank correlation: $t=0.01$ (non significant)

TABLE VI.6: RELATIONSHIP BETWEEN PRESENCE OF ANAEROBES AND LEUCOCYTE COUNT

\begin{tabular}{cccc}
\hline $\begin{array}{c}\text { Leucocyte } \\
\text { count }\end{array}$ & $\begin{array}{c}\text { Anaerobes } \\
\text { present }\end{array}$ & $\begin{array}{c}\text { Anaerobes } \\
\text { absent }\end{array}$ & Total \\
$1-20$ & 12 & 2 & 14 \\
20 & 22 & 16 & 38 \\
Total & 7 & 4 & 63 \\
\hline
\end{tabular}

Wilcoxon rank sum test showed non significant correlation $(z=-1.07)$

TABLE VI.7: RELATIONSHIP BETWEEN UREAPLASMA CULTURE AND LEUCOCYTE COUNT IN INFERTILE MEN $(\mathrm{n}=60)$

\section{Ureaplasma culture}

Number of leucocytes No. of patients per field
No. of patients (\% of -ve)
$1-20$
$2(22.2)$
$5(55.5)$
$2(22.2)$
$12(23)$
30 (57)
20
11 (21) 
TABLE VI.8:

RELATIONSHIP BETWEEN LEUCOCYTE COUNTS AND SPERM ANTIBODIES

\begin{tabular}{ccc}
\hline & \multicolumn{2}{c}{ MAR IgG versus PMN leucocytes } \\
\cline { 2 - 3 } MAR IgG & Number of patients & $\begin{array}{c}\text { PMN leucocytes } \\
\text { (mean } \pm \text { S.D.) }\end{array}$ \\
negative & 14 & $13.2(22.0)$ \\
positive & 5 & $24.8(16.3)$ \\
\hline
\end{tabular}

Serum TAT versus total leucocytic count

Leucocytes

Serum TAT Number of patients (mean \pm S.D.)

$\begin{array}{lll}\text { negative } & 36 & 12.7(16.4)\end{array}$

$\begin{array}{lll}\text { positive } & 5 & 15.7(19.2)\end{array}$ 


\section{VI.G RELATIONSHIP OF LEUCOCYTES TO SPERM DENSITY, MOTILITY AND}

\section{SUBSEQUENT FERTILITY}

There was no correlation between the leucocyte count and the duration of involuntary infertility, sperm motility and sperm density (Kendal rank correlation of $-0.03,0.07$ and 0.05 respectively, non significant (NS)). Furthermore, using the couple-month analysis method, there was no significant relationship between leucocyte counts and subsequent fertility (Tables VI.9 and 10).

\section{VI.H DISCUSSION}

Monoclonal antibodies with specificity to human leucocyte surface markers were used in an indirect immunoperoxidase technique to stain cytocentrifuge smears of washed ejaculate. This immunocytochemical technique has permitted precise identification of leucocytes in the semen. This procedure, which utilizes specific monoclonal antibody probes, allowed further classification and characterization of the leucocytes into their subpopulations.

The technique has many advantages, mainly its high specificity. For example, the antihuman leucocyte monoclonal antibody (HLC-1) has been shown to react with more than $95 \%$ of lymphocytes, monocytes, polymorph nuclear cells, and eosinophils in peripheral blood, spleen and tonsils (Beverley, 1980). Furthermore, in the study on the frozen sections of human peripheral testis (Chapter 4 , section $A$ ) we have shown that the antibodies used 


\section{TABLE VI.9:}

RELATIONSHIP BETWEEN NUMBER OF BACTERIAL SPECIES PER SEMEN SAMPLE AND SPERM MOTILITY

\begin{tabular}{ccccc}
\hline $\begin{array}{c}\text { No. of bacterial } \\
\text { species isolated } \\
\text { per patient }\end{array}$ & \multicolumn{2}{c}{ No. of patients with sperm motility } \\
\hline 0 & 2 & $11-60$ & 60 & Total \\
1 & 2 & 1 & 2 & 5 \\
2 & 3 & 4 & 7 & 13 \\
3 & 4 & 4 & 4 & 11 \\
4 & 2 & 6 & 7 & 17 \\
5 & 1 & 1 & 3 & 12 \\
6 & 1 & 0 & 1 & 2 \\
Total & 15 & 23 & 25 & 63 \\
\hline
\end{tabular}

Kendall rank correlation: $t=0.07$ (NS)

TABLES VI.10:

(a) RELATIONSHIP OF TOTAL LEUCOCYTE COUNTS TO SUBSEQUENT FERTILITY

Pan leucocyte count Observed pregnancies Expected pregnancies

\begin{tabular}{rrrrr}
\hline & & & & \\
0 & 10 & 2 & 1.47 \\
10 & 20 & 4 & 3.94 \\
& 20 & 1 & 2.24 \\
& 20 & 1.35 \\
\hline
\end{tabular}

$\mathrm{X} 2=2.6 \quad 3$ d.f. $\quad(\mathrm{NS})$

(b) RELATIONSHIP OF T-LYMPHOCYTES TO SUBSEQUENT FERTILITY

\begin{tabular}{ccc}
\hline T-1ymphocyte count & Observed pregnancies & Expected pregnancies \\
\hline 0 & 5 & 7.11 \\
0 & 4 & 1.89 \\
\hline
\end{tabular}

$$
X 2=2.99 \quad 1 \text { d.f. } \quad(N S)
$$


in this study do not react with the germinal epithelium, sperm precursors or mature sperms. Therefore, with this immunoperoxidase technique the staining was very specific, and the danger of false negative and false positive results was circumvented by simultaneous study of known positive and negative controls.

It should be stressed that the use of diaminobenzidine/ hydrogen peroxide substrate in this technique may allow visualisation of the peroxidase positive granules, if still active, in the neutrophils, thus allowing simultaneous exploration of currently used techniques to identify this cell population. It also should be noted that the morphology of cells in the fixed smears may be examined simultaneously by light microscopy following staining. The stained preparations provide a permanent record which can be stored at room temperature, and the use of high dilutions of the monoclonal antibodies (1/100-1/160) makes this technique relatively inexpensive. Moreover, the total time required for the labelling procedure was approximately two and a half hours and at least 24 cell smears could be processed in one batch.

In the present study PMN leucocytes were the predominant cell type of leucocytes in the ejaculate in both fertile and subfertile groups. T-1ymphocytes were demonstrated only in 13/63 specimens from subfertile patients, and these lymphocytes represented $28.1 \%$ of the total leucocyte count in these 13 patients. This is in contrast to the findings of Couture et al. (1976) In their study, 1ymphocytes predominated and represented $66.6 \%$ of the total count of leucocytes, while PMN leucocytes represented only $33 \%$ 
of the total count. A possible explanation of the difference in results is that, in Couture's study, round immature germ cells in which the acrosomal cap staining was deficient or difficult to visualize, were counted as lymphocytes, thus increasing their number. It is also possible that the PMN leucocytes which failed to show peroxidase positive granules were considered to be non-separated spermatids, thus lowering the number of PMN leucocytes.

Our finding that the fertile men had large numbers of PMN leucocytes compared with subfertile men raises many questions about the normal role of these cells in the ejaculate. The primary role of PMN leucocytes is internalization and killing of microorganisms by the accumulation of toxic products within phagocytic vacuoles. However, extracellular release and cytotoxicity of these agents, including reactive oxygen derivatives and products of the myeloperoxidase system, are well documented (Clark, 1983). So the existence of PMN leucocytes in the ejaculate may provide a non specific and rapid defence mechanism and protection against invading organisms. The presence of these cells often is used as a criterion of subclinical genital infection, but perhaps these cells have another role.

It is well known that during spermiogenesis, excess spermatid cytoplasm is shed as residual bodies (Clermont, 1970). These cytoplasmic fragments are phagocytosed, broken down, and subsequently resorbed by Sertoli cell 1ysosomes. A small portion of cytoplasm, the cytoplasmic droplet, however, remains attached to the midpiece of spermatozoa. So, it is possible that the PMN-leucocytes in the ejaculate have a role in the final 
phagocytosis of the residues of degenerating germ cells and of residual bodies in the ejaculate. On the other hand, subfertile men have a high percentage of abnormal sperm forms which would, perhaps, result in an over stimulation of phagocytosis but for the presence of suppressor/cytotoxic cells.

In this study we tried to find a possible relationship between subclinical genital tract infection and male infertility. We defined precisely the leucocytes and leucocyte subpopulations and also performed cultures for a range of microorganisms in the ejaculate studied. A high bacterial isolation rate was found: $87 \%$ of the semen cultures of both the fertile and infertile groups were positive for microorganisms with no difference between the patients and fertile groups. However, there was no significant correlation between leucocyte counts and the counts of positive cultures for all microorganisms. The significant correlation between B-streptococci and $T$-1ymphocytes $(P<0.05)$ is difficult to explain but may be a spurious result from multiple statistical tests. Furthermore, no correlations were found between leucocytes and sperm density, motility and subsequent fertility.

In 1981 Fowler reviewed the literature and found little evidence to support infection as a frequent cause of infertility Our results are also in agreement with those of other studies. Naessens et al. (1986) reported that $97.7 \%$ of the semen cultures were positive for aerobes and anaerobes and they found that no single aerobic or anaerobic organism could be related to abnormal semen samples. Comhaire et al. (1980) found no significant difference in semen characteristics of infertile males with and without infection. McGowan et a1. (1981) found that infection in 
the semen did not significantly affect the count, motility or volume of the specimen. In his experience with 290 patients, Hendry (1983) found that pregnancies were produced with roughly equal frequency with or without positive semen cultures irrespective of whether the organisms were treated with antibiotics or not.

Our observation that none of the controls and the infertile men was positive for Chlamydia may be due to the fact that all men examined were asymptomatic and had no history of specific or non specific genital tract infections and Chlamydia is not a commensal organism. However, Ulstein et al. (1976) were unable to culture Chlamydia from the urine, expressed prostatic secretion or semen of 21 asymptomatic men with unexplained infertility.

Our results showed no correlation between ureaplasma culture results and leucocyte response. These results are in agreement with those published by Upadhyaya et al. (1984) who also studied an asymptomatic population of men. It has been suggested that the presence of ureaplasma in the semen is merely the result of contamination at the time of ejaculation (Deasi et al., 1980). De Louvois et al. (1974), Ulstein et a1. (1976) and Matthew et al. (1975) found no differences in the frequency of ureaplasma from the genital tract of infertile men and fertile control subjects. In addition, Hargreave et al. (1982c) have not found any relationship between ureaplasma isolated from seminal plasma and the occurrence of sperm antibodies.

The positive correlation between the PMN-leucocyte count and the direct MAR IgG seems to be a spurious result from the statistical analysis. This is roughly what might be expected as a chance finding in 25 correlations. However, the lack of correlation with serum TAT seems to be a fairly definite finding. 


\section{VI.I CONCLUSION}

The use of specific monoclonal antibodies in conjunction with the indirect immunoperoxidase technique enabled precise identification of leucocytes and leucocyte subpopulations in ejaculate smears. This suggests that the immunocytochemical technique, as described in this study, is a suitable means of screening semen specimens for the presence of leucocytes.

The evidence from both the leucocyte and bacterial studies suggests that subclinical genital tract infection in asymptomatic patients has no major role in male infertility. We found no correlation between sperm density, sperm motility, the presence of sperm antibodies, positive bacterial cultures and the leucocyte response in the semen. These results cast doubts on the currently accepted criteria of subclinical genital tract infection. 
CHAPTER VII

CONCLUDING DISCUSSION 
This is the first systematic immunocytochemical study of lymphocyte subsets and macrophages in the male genital tract. It provides us with the first clear picture of the distribution of immunocompetent cells within various tissues of the normal male genital tract and its secretions. This distribution appears to be remarkably consistent, the main observations being as follows:-

1) T-1ymphocytes are the predominant cell type in all tissues examined apart from the peripheral testis where none were identified.

2) The T-cells present are largely of the suppressory cytotoxic phenotype.

3) There is a specific pattern of distribution of T-cells within the various tissues, the suppressor/cytotoxic T-cells being more abundant within the epithelium and lamina propria, while T-cells of the helper/inducer phenotype are found mainly within the interstitium.

4) T-1ymphocytes were found to represent $10.5 \%$ of the mucosal cell population of the epithelium.

5) Macrophages were detected in high numbers in the peripheral testis and are also found throughout the genital tract.

6) B-cells are found in appreciable numbers only in the prostate.

7) Natural killer cells are present in the prostate.

8) Polymorph nuclear leucocytes were the predominant cell type in the ejaculate.

Of added interest was the marked pertubations in the incidence, distribution and possible state of activation (i.e. IL2 receptor expression) of cells of the immune system noted in a 
variety of clinical disorders, especially testicular obstructions and chronic prostatitis.

The patterns of distribution observed in normal specimens can act as a basis for future studies and can be readily explained in terms of the functional properties of the genital tract, while the changes noted in clinical biopsies are also consistent with the observed clinical and laboratory findings. Together they provide a cohesive picture of the importance of the local immune system to the effective functioning and protection of the male genital tract.

of particular interest is the preponderance of suppressor-cytotoxic T-cells within the epithelium and lamina propria of those tissues where the blood-testis barrier is weak or deficient, or luminal contents are absorbed and sperm are stored. It is not unreasonable to suggest that one of the key functions of the suppressor cell component of this population is to limit an autoimmune response to the differentiation antigens expressed upon the germ cells. In this context it should be noted that there is substantial evidence indicating that suppressor T-cells are involved in inducing tolerance to self antigens (Cowing, 1985). Our findings on the distribution of HLA-DR molecules on the epithelia of the rete testis, epididymis and vas deferens are also of interest in view of the observation that tolerance is Class II restricted (Lowy et a1., 1984).

While the absence of 1ymphocytes from the peripheral testis could be explained on the basis that tight junctions prevent exposure of the immune system to potentially autoantigenic sperm antigens, the presence of relatively large numbers of cells reacting with antimonocyte/macrophage antibodies was unexpected in the light 
of previous observations using conventional histological techniques. However, our observations are in agreement with previous histological studies in man (Holstein, 1978) and rats (Miller et al., 1984). In addition the rat testis has recently been shown to produce large amounts of interleukin-1 which is known to be a macrophage-derived protein (Khan et al., 1987).

The role of these macrophages in testicular tissues is not clear. They may provide an ancillary back-up protection against the risk of escaped sperm antigens following alterations in the integrity of the blood-testis barrier. The cells observed elsewhere in the genital tract could perform a direct phagocytic function against debris or sperm breakdown products. Alternatively they could also participate in the complex cellular interactions known to occur in both cellular or humoral immune responses by acting either as antigen-presenting cells (Friedman et al., 1981) or producing essential soluble factors such as ILl. Whether or not these cells are functioning as phagocytic or antigen-presenting cells, or in some other manner, remains to be established.

In certain respects the prostate was unique. While it contained large numbers of $\mathrm{T}$ cells and macrophages like other genital tract tissues, $\mathrm{B}-\mathrm{cell}$ s and $\mathrm{NK}-\mathrm{cells}$ were relatively more abundant. The presence of more B-cells is probably not surprising in view of the known local production of immunoglobulin by the prostate (Krieger, 1984). However the reason for the preferential localisation of $\mathrm{NK}$-cells is less apparent. Nevertheless it is interesting to note that the functions recently ascribed to NK-cells could be highly relevant to maintenance of this tissue. These include its ability to inhibit the proliferation of both malignant 
and normal cells, especially B-cells (James and Ritchie, 1984) and its antiviral activity.

Our studies on the distribution of 1ymphocyte subsets in patients with testicular obstruction have proved particularly illuminating. Of special interest are the apparent associations between the distribution of the various $\mathrm{T}$-cell subsets and the development of antisperm antibodies. Thus the presence of high levels of antisperm antibodies, as noted in patients with unilateral obstruction and post-vasectomy patients, is accompanied by T-helper cell infiltration of the testis and a decline in the T-suppressor cell content of the epithelia and lamina propria of the epididymis. In contrast, in patients with obstructive azoospermia who had low levels of antisperm antibody, the lymphocytes infiltrating the testis were of the suppressor cytotoxic phenotype. These observations may explain the origin of sperm antibodies, and suggest the presence of antisperm cell-mediated reactions or indicate that the distinctive distribution of suppressor cells in the epithelium may exist in order to prevent the development of an autoimmune response to tissue specific differentiation antigens present in sperm.

One interesting feature of this study was the finding that polymorphonuclear leucocytes were the predominant cell type in the ejaculate and that the fertile men had large numbers of these cells compared with patients with an infertile marriage. In addition there was no correlation between sperm density, sperm motility, the presence o rakgraly. the leucocyte response in the semen. The inference from this finding is that these cells may have a further role in the ejaculate 
other than being a criterion of infection, for example elimination of sperm debris, residues of degenerating germ cells, and dead and/or immature sperm.

The identification of T-1ymphocyte subsets and macrophages in normal urothelium suggests a probable common pattern of deployment of immune competent cells within mucosal surfaces and perhaps a shared mucosal immunity system. These cell populations should also be considered in relation to the understanding of mucosal immunity in general.

There are a number of ways in which the present work could be extended. It is patently obvious that the results obtained by these studies need to be confirmed in a large series. Of prime importance in this regard are similar investigations in patients with detectable sperm antibodies in serum and/or semen. Further studies on other genital tract disorders, e.g. chronic prostatitis or infections of the genital tract, are essential. Where possible, further studies with other monoclonal probes would be an advantage, especially those which indicate the activational status of cells of the immune system. Undoubtedly however, the most pressing need is for studies with a monoclonal which can readily distinguish between the T-suppressor and T-cytotoxic cell subsets. Furthermore, functional studies are also essential but at the present time the availability of tissue, together with technical limitations, render this difficult. Finally, extension of these studies to both normal foetal and prepubertal male tissue and tissues of the female genital tract should provide information of both theoretical and practical importance. In this context it would enable us to establish if the characteristic localisation of T-suppressor cells in the male genital tract precedes puberty. 


\section{Acknowledgements}

The work in this thesis was performed within the Department of Surgery, University of Edinburgh, between January 1984 and April 1987.

A number of people have helped me in various ways with this work. I am particularly grateful to my supervisors Dr Keith James and $\mathrm{Mr} \mathrm{T} . \mathrm{B}$. Hargreave for giving up so much of their time for guidance, help, discussion and constructive criticism. I am also greatly indebted to Professor G.D. Chisholm for giving me the opportunity to perform this work in his department and for his genuine encouragement.

The practical help of a number of other people is also acknowledged: Dr A. Busuttil (Department of Pathology, Western General Hospital, Edinburgh) for his advice and assistance in histopathological assessments and interpretation; Dr H. Young (Department of Bacteriology, University of Edinburgh) for conducting the bacterial cultures and $\operatorname{Dr}$ R. Elton (Medical Statistics Unit, University of Edinburgh) for help with statistical analysis. In addition, I would like to thank Mr F. Cochrane and Mr I. Milne for their help.

Finally, this study would not have been possible without the invaluable help and encouragement of Dr Keith James. 
BIBLIOGRAPHY 
ABLIN, R.J., SOAMES, W.A. and CONDER, M.J. (1972) In vivo bound immunoglobulin in the human prostate - their identification and possible significance. Immunito-etsforsch, 144:233.

ABNEY, E.R., COOPER, M.D., KEARNEY, J.F., LAWTON, A.R. and PARKHOUSE, R.M.E. (1978) Sequential expression of immunoglobulin on developing mouse B-1ymphocytes. A systematic survey which suggests a model for the generation of immunoglobulin isotype diversity. Journal of Immunology, 120:2041.

ABO, T. and BALCH, C.M. (1981) A differentiation antigen on human NK and $\mathrm{K}$ cells identified by a monoclonal antibody (HNK-1). Journal of Immunology, 127:1024.

ABO, T., MILLER, C.A. and BALCH, C.M. (1984) Characterization of human granular 1ymphocyte subpopulations expressing HNK-1 (leu 7) and leu 11 antigens in the blood and lymphoid tissues from fetuses, neonates and adults. European Journal of Immunology, 14:616.

ALEXANDER, N.J. and ANDERSON, D.J. (1979) Vasectomy: consequences of autoimmunity to sperm antigens. Fertility and Sterility, $32: 253$.

ALLEN, C. and HOGG, N. (1985) Monocytes and other infiltrating cells in human colorectal tumours identified by monoclonal antibodies. Immunology, 55:289.

AMANN, R.P. and HOWARDS, S.S. (1980) Daily spermatozoal production and epididymal spermatozoal reserves of the human male. Journal of Urology, $124: 211$.

AUMULLER, G. (1983) Morphology and endocrine aspects of prostatic function. Prostate, 4:195.

BARNES, G.W., SHULMAN, S., CONDER, J.M. and SOAMES, W.A. Journal of Laboratory and Clinical Medicine, 66:741.

BASHAM, T.J. and MERICAN, T.C. (1983) Recombinant interferon-y increased HLA-DR synthesis and expression. Journal of Immunology, 130:1492.

BEER, A.E. and NEAVES, W.B. (1978) Antigenic status of semen from the viewpoints of the female and male. Fertility and Sterility, $29: 3$.

BELL, E.B. and McLAREN, A. (1979) Reduction of fertility in female mice iso-immunized with a subcellular sperm fraction. Journal of Reproduction and Fertility, 22:345. 
BELSEY, M.A.,ELIASSON, R., GALLEGOS, A.J., MORGHISSI, K.S., PAULSEN, C.A. and PRAVAL, M.R.N. (eds.) (1980) WHO Laboratory Manual for the Examination of Human Semen and Cervical Interaction, pp.9-13. Distributed by Special Program Research Development and Research Training in Human Reproduction. Geneva: World Health Organisation.

BENACERROF, B. (1981) Role of MHC gene products in immune regulation. Science, 212: 1229.

BERGH, A. (1985) Effect of cryptorchidism on the morphology of testicular macrophages: evidence for a Leydig cell - macrophage interaction in the rat testis. International Journal of Andrology, 8:89.

BERGROTH, V., REITAMO, S. KONTINNEN, Y.T. and LALLA, M. (1980) Sensitivity and non specific stain of various immunoperoxidase techniques. Histochemistry, 68:17.

BEVERLEY, P.C.L. (1980) Production and use of monoclonal antibodies in transplantation immunology. In: J.D.L. Touraine, J. Traeger, H. Betuel, J. Brochier, J.M. Dufernand, J.P. Revilland and R. Triau (eds.): Transplantation and Clinical Immunology XI, pp.87-94. Amsterdam: Excerpta Medica.

BIENENSTOCK, J., JOHNSTON, N. and PERCY, D.Y.E. (1973) Bronchial 1ymphoid tissue. 1. Morphologic characteristics. Laboratory Investigation, 28:686.

BIENENSTOCK, J., BEFUS, D. and McDERMOTT, M. (1980) Mucosal immunity. Monographs in Allergy, 16:1.

BIGAZZI, P.E., ALEXANDER, N.J. and SILBER, S.J. (1979) Studies on testicular biopsies from vasectomised men. In: I.H. Lepow and R. Crozier (eds.): Vasectomy: Immunologic and Pathophysiologic Effects in Animals and Man, p.509. New York: Academic Press.

BLAND, P.W. and WARREN, L.G. (1986) Antigen presentation by epithelial cells of the rat small intestine. I. Kinetics, antigen specificity and blocking by anti-Ia antisera. Immunology, 58:1.

BOETTCHER, B., KAY, D.J., RIIMKE, P.H. and WRIGHT, L.E. (1971) Human sera containing immunoglobulin and non-immunoglobulin spermagglutinins. Biology of Reproduction, 5:236.

BOORSMA, D.M., STREEFKERK, D.-F. and KORS, N. (1976) Peroxidase and fluoroscein isothiocyanate as antibody markers. A quantitative comparison of two peroxidase conjugates prepared with gluteraldehyde or periodate and a fluorescein conjugate. Journal of Histochemistry and Cytochemistry, 24: 1017.

BOSMAN, F.F. (1983) Some recent developments in immunocytochemistry. Histochemical Journal, 15, 189. 
BOYUM, A. (1968) Separation of leucocytes from blood and bone marrow. Scandinavian Journal of Clinical and Laboratory Investigation, 21: Supp1. 97.

BRYAN, J.H.D. (1970) An eosin-fast green naphthol yellow mixture for differential staining of cytologic components in mammalian spermatozoa. Stain Technology, 45:231.

BUSTOS-OBREGON, E. (1976) Ultrastructure and function of the lamina propria of mammalian seminiferous tubules. Andrologia, $8: 179$.

BUTCHER, E.C. and WEISSMAN, I.L. (1984) Lymphoid tissues and organs. In: W.E. Paul (ed.) Fundamental Immunology, Ch. 6, pp.109-127. Raven Press, New York.

BUXTON, C.L. and SOUTHAM, A.I. (1958) Human Infertility. London: Casse11, 7.

CANTOR, H. and BOYSE, E.A. (1975) Functional subclasses of T lymphocytes bearing different Ly antigens. I. Generation of functionally distinct $\mathrm{T}$-cell subclasses in a differentiative process independent of antigen. Journal of Experimental Medicine, 141:1376.

CHRISTENSEN, A.K. (1975) Leydig cells. In: D.W. Hamilton, and R.0. Greep (eds.), Male Reproductive System, pp.57-94. American Physiological Society, Washington DC. (Handbook of Physiology, Sect. 7, Vol. 5).

CLARK, R.A. (1983) Extracellular effects of the myeloperoxidase hydrogen peroxide halide system. In: G.L. Weissman (ed.) Advances in Inflammatory Research, vol. 5, p.107. New York: Raven Press.

CLAVERT, A., GABRIEL-ROBEZ, O. and MONTAGNON, D. (1985) Physiological role of seminal vesicle in seminal vesicles and fertility. Progress in Reproductive Biology and Medicine, $12: 80$.

CLEMENT, L.T., DAGG, M.K. and LANDAY, A. (1984) Characterization of human lymphocyte subpopulations: alloreactive cytotoxic T-1ymphocyte precursor and effector cells are phenotypically distinct from Leu $2^{+}$suppressor cells. Journal of Clinical Immunology, 4:395.

CLERMONT, Y. (1963) The cycle of the seminiferous epithelium in man. American Journal of Anatomy, 112:35.

CLERMONT, Y. (1970) Dynamics of human spermatogenesis. In: E. Rosenberg and C.A. Paulsen (eds.) The Human Testis, p.47. New York: Plenum Press.

CLERMONT, Y. (1972) Kinetics of spermatogenesis in mammals. Seminiferous epithelium cycle and spermatogonial renewal. Physiology Review, 52:198. 
COMHAIRE, E., VERSCHREAGEN, G. and VERMEULEN, L. (1980) Diagnosis of accessory gland infection and its possible role in male infertility. International Journal of Andrology, $3: 32$.

COMMITTEE ON HUMAN LEUCOCYTE DIFFERENTIATION ANTIGENS: IUIS-WHO NOMENCLATURE SUBCOMMITTEE (1984) Differentiation human leucocyte antigens: a proposed nomenclature. Immunology Today, $5: 158$.

CONNELL, C.J. and CONNELL, G.M. (1977) The interstitial tissue of the testis. In A.D. Johnson and W.R. Gomes (eds.), The Testis, Vol. IV, pp.333-370. New York: Academic Press.

COONS, A.H., CREECH, H.J. and JONES, R.N. (1941) Immunological properties of an antibody containing a fluorescent group. Proceedings of the Society of Experimental Biology, 47:200.

COOPER, M.D., KEARNEY, J.F., GATHINGS, W.E. and LAWTON, A.R. Effects of anti-Ig antibodies on the development and differentiation of B cells. Immunological Reviews, Vol. 52:29.

COOPER, T.G. (1986): Function of the epididymis and its secretory products. In: T.G. Cooper (ed.) The Epididymis, Sperm Maturation and Fertilisation, pp.117-139. New York: Springer-Verlag.

COUTURE, M., ULSTEIN, M., LEONARD, J. and PAULSEN, C.A. (1976) Improved staining method for differentiating immature germ cells from white blood cells in human seminal fluid. Andrologia, $8: 61$.

COWAN, S.T. (1974) Cowan and Steel's Manual for the Identification of Medical Bacteria. 2nd ed. Cambridge: Cambridge University Press.

COWING, C. (1985) Does $\mathrm{T}$ cell restriction of Ia limit the need for self tolerance? Immunology Today, 6:72.

D'ALMEIDA, M., LEFROIT-JOLLY, M. and VOISIN, G.A. (1981) Studies on human spermatozoa auto-antigens. I. Fractionation of sperm membrane antigens: evidence of three antigenic systems. Clinical Experimental Immunology, 44:359.

de KRETSER, D.M. and BURGER, H.G. (1972) U1trastructural studies of human Sertoli cells in normal men and males with hypogonadotrophic hypogonadism before and after gonadotiophic treatment. In: B.B. Saxena, C.G. Beling and H.M. Gandy (eds.), Gonadotrophins, pp.640-656. New York: Wiley.

De LOUVOIS, J., HARRISON, R.F., BLADES, M., HURLEY, R. and STANLEY, V.C. (1974), Frequency of mycoplasma in fertile and infertile couple. Lancet 1:1073.

DESAI, S., COHEN, M.S., KHARAMEE, M. and REITER, E. (1980) Ureaplasma urealyticum ( $T$-mycoplasma) infection: does it have a role in male infertility? Journal of Urology, 124:469. 
De WAELE, M., De MEY, J., MOEREMANS, M., De BRABANDEN, M. and Van CAMP, B. (1983) Immunogold staining method for the detection of cell surface antigens with monoclonal antibodies. In: G.R. Bullock and P. Petrusz (eds.), Immunocytochemistry, pp. 1-23. London: Academic Press.

DIMITRIU-BONA, A., BURMESTER, G.R., WATERS, S.J. and WINCHESTER, R.J. (1983) Human mononuclear phagocyte differentiation antigens. I. Patterns of antigenic expression on the surface of human monocytes and macrophages defined by monoclonal antibodies. Journal of Immunology, 130:145.

DIXON, W.J. (1985) BMDP Statistical Software. Berkeley: University of California Press.

DONDERO, F., LENZI, A., PICARDO, M., PASTORE, R. and VALESINI, G. (1980) Cell mediated antisperm immunity in selected forms of male infertility. Andrologia, 12:25.

DONDERO, F. (1982) Cel1 mediated antisperm immunity in male infertility. In: S. Shulman, F. Dondero and M. Nicotra (eds.), Immunological Factors in Human Production, pp.92-97. London: Academic Press.

DUBOIS-DALQ, M., McFARLAND, H. and McFARLIN, D. (1977) Protein A peroxidase: a valuable tool for the localisation of antigens. Journal of Histochemistry and Cytochemistry, 25:1201.

DUERDEN, B.I., COLLEE, J.G., BROWN, R., DEACON, A.G. and HOLBROOK, W.P. (1980) A scheme for the identification of clinical isolates of Gram negative anaerobic bacilli by conventional bacteriological tests. Journal of Medical Microbiology, 13:231.

DYM, M. and FAWCETT, D.W. (1970) The blood testis barrier in the rat and the physiological compartmentation of the seminiferous epithelium. Biology of Reproduction, 3:308.

DYM, M. and ROMRELL, L.J. (1975) Intraepithelial 1ymphocytes in the male reproductive tract of rats and rhesus monkeys. Journal of Reproductive Fertility, 42:1.

EDDY, E.M., VERNON, R.B., MULLER, C.H., HAHNEL, A.C., FENDERSON, B.A. (1985) Immunodissection of sperm surface modification during epididymal maturation. American Journal of Anatomy, $174: 225$.

EDWARDS, R.G. (1964) Immunological control of fertility in female mice. Nature, 203:50.

EL-ALFI, O.S. and BASSILI, F. (1970) Immunological aspermatogenesis in man. I. Blastoid transformation of lymphocytes in response to seminal antigen in cases of non-obstructive azoospermia. Journal of Reproduction and Fertility, 21:23. 
ELIASSON, R., FREDRICSSON, B., JOHANNISSON, E. and LEANDER, G. (1967) Biochemical and morphological changes in semen from men with diseases in the accessory genital glands. Excerpta Medica International Congress Series, 133:625.

ELIASSON, R. and JOHANNISSON, E. (1978) Cytological studies of prostatic fluids from men with and without abnormal palpatory findings of the prostate. II. Clinical application. International Journal of Andrology, 1:582.

ENDTZ, A.W. (1972) Ein methode om het vochtige urinesediment en het vochtige menselijke sperma rechtst reeks et kleuren. Neel Tijelschr Geneeskd, 116:681.

ENGLEMAN, E.G., BENIKE, C.J., GLICKMAN, E. and EVANS, R.L. (1981) Antibodies to membrane structures that distinguish suppressor/ cytotoxic and helper T-lymphocyte subpopulations that block the mixed leucocyte reaction in man. Journal of Experimental Medicine, 154:193.

ERBER, W.N., PINCHING, A.J. and MASON, D.Y. (1984) Immunocytochemical detection of $\mathrm{T}$ and $\mathrm{B}$ cell populations in routine blood smears. The Lancet, 1042-5.

ERICKSON, R.P., FRIEND, D.S. and TENNENBAUM, D. (1975)

Localization of lactate dehydrogenase-X on the surface of mouse spermatozoa. Experimental Cell Research, 91:1.

EVANS, R.L., LAZARUS, H., PENTA, A.C. and SCHLOSSMAN, S.F. (1978) Two functionally distinct subpopulations of human $\mathrm{T}$ cells that collaborate in the generation of cytotoxic cells responsible for cell mediated 1ympholysis. Journal of Immunology, 129:1423.

EVANS, R.L., WALL, D.W., PLATSOUCAS, C.D., SIEGAL, F.P., FIKRIG, S.M., TESTA, C.M. and GOOD, R.A. (1981) Thymus-dependent membrane antibens in man: inhibition of cell mediated 1ymphocytosis by monoclonal antibodies to the TH2 antigen. Proceedings of the National Academy of Science, 78:544.

FABBRINI, A. and HAFEZ, E.S.E. (1980) Testes and epididymis. In: E.S.E. Hafez (ed.), Human Reproduction, Conception and Contraception, 2nd ed., pp.35-59. London: Harper and Row.

FALINI, B. and TAYLOR, C.R. (1983): New developments in immunoperoxidase techniques and their application. Archives of Pathology and Laboratory Medicine, 107:105.

FAWCETT, D.W. (1975) U1trastructure and function of the Sertoli cell. In: R.0. Greep and D.W. Hamilton (eds.), Handbook of Physiology, Sect. 7, Vo1. 5, pp.21-55. Baltimore: Williams and Wilkins.

FERGUSON, A. (1977) Intraepithelial 1ymphocytes of the small intestine. Gut, 18:921. 
FERRANTE, A. and THONG, Y.H. (1980) Optimal conditions for simultaneous purification of mononuclear and polymorphonuclear leucocytes from human peripheral blood by the Hypaque-Ficoll method. Journal of Immunological Methods, 36:109.

FJALLBRANT, B. (1965) Immune agglutination of sperm in cases of sterility. Acta Obstetricia et Gynecologica Scandinavica, $44: 474$.

FJALLBRANT, B. (1969) Studies on sera from men with sperm antibodies. Acta Obstetricia et Gynecologica Scandinavica, 48:131.

FJALLBRANT, B. and NILSSON, S. (1977) Decrease of sperm antibody titer and conception after treatment of chronic prostatitis (Abstr.), Fertility and Sterility, 28:317.

FJALLBRANT, B. and OBRANT, 0. (1968) Clinical and seminal finding in men with sperm antibodies. Acta Obstetricia et Gynecologica Scandinavica, $47: 451$.

FLOCKS, R.H., URICH, V.C., PATEL, C.A. and OPITZ, J.M. (1960) Studies on the antigenic properties of prostatic tissue. International Journal of Urology, 10:134.

FOWLER, J.E. (1981) Infections of the male reproductive tract and infertility: a selected review. Journal of Andrology, 3:121.

FRANKLIN, R.R. and DUKES, C.D. (1964) Antispermatozoal antibody and unexplained infertility. American Journal of Obstetrics and Gynecology, 89:6.

FREUND, M.J. and DAVIS, J.E. (1969) Disappearance rate of spermatozoa from the ejaculate following vasectomy. Fertility and Sterility, 20:163.

FRIBERG, J. (1974) A simple and sensitive micro method for demonstration of sperm agglutinating activity in serum from infertile men and women. Acta Obstetricia et Gynecologica Scandinavica, Supp1. 36: $2 \overline{1}$.

FRIEDMAN, S.M., HUNTER, S.B., IRIGOYEN, O.H., KUNG, P.C., GOLDSTEIN, G. and CHESS, R. (1981) Functional analysis of human $\mathrm{T}$ cell subsets defined by monoclonal antibodies. II. Collaborative T-T interactions in the generation of TNPaltered-selfreactive cytotoxic T-1ymphocytes. Journal of Immunology, 126:1702.

GALLYAS, F., GORCS, T. and MERCKENTHALLER, I. (1982) High grade intensification of the end product of the diaminobenzidine reaction for peroxillase histochemistry. Journal of Histochemistry and Cytochemistry, 30:183.

GARDINER, R.A., SEYMOUR, G.J., LAVIN, M.F., STRUTTON, G.M., GEMMELL, E. and HAZAN, G. (1986) Immunohistochemical analysis of the human bladder. British Journal of Urology, 58:19. 
GRAHAM, R.C., Jr.and KARNOVSKY, M.J. (1966) The early stages of absorption of injected horseradish peroxidase in the proximal tubules of mouse kidney. Ultrastructural cytochemistry by a new technique. Journal of Histochemistry and Cytochemistry, 14:291.

GRANT, G.H.and EVERALL, P.H. (1963) Protides of the biological fluids. In: H. Peeters (ed.), vol. 10, p.237. Amsterdam/ London/New York: Elsevier.

GUAZZIERIO, S., LEMBO, A., FERRO, G. , ANTIBANI, W., MERLO, F. , ZANCHETTO, R. and PAGANO, F. (1985) Sperm antibodies and infertility in patients with testicular cancer. Urology, $26: 139$.

GUESDON, J.L. TERNYNCK, T. and AURAMEAS, S. (1979) The use of avidin-biotin interaction in immunoenzymatic techniques. Journal of Histochemistry and Cytochemistry, 27:1131.

GREENWOOD, J.H., AUSTIN, L.L. and DOBBINS, W.O. (1983) III. In vitro characterization of human intestinal intraepithelial lymphocytes. Gastroenterology, 85:1023.

HAAS, G.G., SOKOLOSKI, J.E. and WOLF, D.P. (1980) The interfering effect of human IgG antisperm antibodies on human sperm penetration of zona-free hamster eggs. American Journal of Reproductive Immunology, 1:40.

HAAS, G.G. and BEER, A.E. (1986) Immunologic influences on reproductive biology: sperm gametogenesis and maturation in the male and female genital tracts. Fertility and Sterility, 5:753.

HAENSCH, R. (1973) Spermatozoen-autoimmunophaenomehe bei genitaltraumen und Verschlubba-zoospermie. Andrologia, 5:147.

HAHN, H. and KAUFMANN, S.H.E. (1981) The role of cell-mediated immunity in bacterial infections. Review of Infectious Diseases, 3:1221.

HALIM, K., WRONG, D.M. and MITTAL, K.K. (1982) The HLA typing of human spermatozoa by two-color fluorescence. Tissue Antigens, 19:90.

HAMERLYNCK, J.V.T.H. (1970) Cytotoxic and other auto-antibodies against spermatozoa in relation to infertility in the human male. Thesis, Amsterdam.

HAMILTON, D.W. (1972) The mammalian epididymis. In: H. Balin and S. Glasser (eds.), Reproductive Biology, pp.268-337. Amsterdam: Excerpta Medica.

HANCOCK, R.J.T. (1978) Sperm antigens and sperm immunogenicity. In: J. Cohen and W.F. Hendry (eds.), Spermatozoa, Antibodies and Infertility, p.1. Oxford: Blackwell Scientific Publications. 
HARGREAVE, T.B., HAXTON, M., WHITELAW, J., ELTON, R. and CHISHOLM, G.D. (1980) The significance of serum spermagglutinating antibodies in men with infertile marriages. British Journal of Urology, 52:566.

HARGREAVE, T.B. (1982a) Incidence of serum agglutinating and immobilizing antibodies in infertile couples. International Journal of Fertility, 27:90.

HARGREAVE, T.B., BUSUTTIL, A., ELTON, R.A., HARVEY, J., CHAN, A. and CHISHOLM, G.D. (1982b) Studies of testicular and epididymal damage in relation to the occurrence of antisperm antibodies. British Journal of Urology, 54:769.

HARGREAVE, T.B., TORRANCE, M., YOUNG, H. and HARRIS, A.B. (1982c) Isolation of Ureaplasma urealyticum from seminal plasma in relation to sperm antibody levels and sperm motility. Andrologia 14, 223.

HELLER, C.H. and CLERMONT, Y. (1964) Kinetics of the germinal epithelium in man. Recent Progress in Hormone Research, 20:545.

HENDRY, W.F., MORGAN, H. and STEDRONSKA, J. (1977) The clinical significance of antisperm antibodies in male subfertility. British Journal of Urology, 49:757.

HENDRY, W.F., STEDRONSKA, J., HUGHES, L., CAMERON, K.M. and PUGH, R.C.B. (1979) Steroid treatment of male subfertility caused by antisperm antibodies. Lancet 2:498.

HENDRY, W.F., STEDRONSKA, J., PARSLOW, J. and HUGHES, L. (1981) The results of intermittent high dose steroid therapy for male infertility due to antisperm antibodies. Fertility and Sterility, 36:351.

HENDRY, W.F., PARSLOW, J.M., STEDRONSKA, J. and WALLACE, D.M.A. (1982) The diagnosis of unilateral testicular obstruction in subfertile males. British Journal of Urology, 54:774.

HENDRY, W.F. (1983) Treatment of antisperm antibodies. In: T.B. Hargreave (ed.), Male Infertility, pp.280-296. Berlin/ Heidelberg/New York/Tokyo: Springer-Verlag.

HENDRY, W.F. (1986) Clinical significance of unilateral testicular obstruction in subfertile males. British Journal of Urology, $58: 709$.

HERBERMAN, R.B. and ORTALDO, J.R. (1981) Natural killer cells: their role in defense against diseases. Science, 214:24.

HEUER, S.C., SCHLOSSMAN, S.F. and REINHERZ, E.L. (1982) Clona1 analysis of human cytotoxic T-lymphocytes $\mathrm{T}^{+}$and $\mathrm{T}^{+}$effector T-cells recognize products of major histocompatibility complex regions. Proceedings of the National Academy of Sciences, $79: 4395$. 
HEYDERMAN, E. and NEVILLE, A.M. (1977) A shorter immunoperoxidase technique for the demonstration of carcinoembryonic antigen and other cell products. Journal of Clinical Pathology, 30:138.

HJORT, T., HUSTED, S. and LINNET-JEPSEN, P. (1974) The effect of testis biopsy on autosensitization against spermatozoal antigens. Clinical and Experimental Immunology, 18:201.

HJORT, T. (1983) Autoimmunity to sperm. In: T.B. Hargreave (ed.), Male Infertility, chapter 9. New York: Springer-Verlag.

HOFFER, A.P., HAMILTON, D.W. and FAWCETT, D.W. (1973) The ultrastructure of the principal cells and intraepithelial leucocytes in the initial segment of the rat epididymis. Anatomical Record, 175:169.

HOFFER, A.P. (1976) The ultrastructure of the ductus deferens in man. Biology of Reproduction, 14:425.

HOGG, N. and SELVENDRAN, Y. (1985) An anti-human monocyte/ macrophage monoclonal antibody reacting most strongly with macrophages in 1ymphoid organs. Cell Immunology, 92:245.

HOGG, N., TAKACS, L., PALMER, D.G., SELVENDRAN, Y. and ALLEN, C. (1986) The P150, 95 molecule is a marker of human mononuclear phagocytes: comparison with expression of class II molecules. European Journal of Immunology, 16: 240-248.

HOLBOROW, E.J. and PAPAMICHAIL, M. (1983) The 1ymphoid system and lymphocyte subpopulations. In E.J. Holborow and W.G. Reeves (eds.) Immunology in Medicine, 2nd ed., Ch.2, pp.18-34. London and New York: Academic Press.

HOLSTEIN, A.F. (1969) Morphologische studien am Nebenhoden des Menschen. Normale und Pathologische Anatomie, 20:1.

HOLSTEIN, A.F. (1976a) Ultrastructural observations on the differentiation of spermatids in man. Andrologia, 8:157.

HOLSTEIN, A.F. (1976b) Structure of the human epididymis. In: E.S.E. Hafez (ed.), Human Semen and Fertility Regulation in Men, pp.23-30. Saint Louis: Mosby.

HOLSTEIN, A.F. (1978) Spermatophagy in the seminiferous tubules and excurrent ducts of the testis in rhesus monkey and in man. Andrologia, 10 (5):331.

HOLSTEIN, C. (1983) Morphology of free immature germ cells in human testis, epididymis, and ejaculate. Andrologia, 15:7.

HORSTMANN, E. (1962) Die elektromemmikroskopie des menschlichen nebenhodenepithels. Z. Zellforsch. Mikrosk. Anat., 57:692.

HSU, S.M. and RAINE, L. (1981) Protein A, avidin and biotin in immunohistochemistry. Journal of Histochemistry and Cytochemistry, 29:1349. 
HUCKINS, C. (1978) The morphology and kinetics of spermatogonial degeneration in normal adult rats: an analysis using a simplified classification of the germinal epithelium. Anatomical Record, 190:905.

HUI, P.K. and LAWTON, J.W.M. (1984) Immunoperoxidase detection of $\mathrm{T}$ and $\mathrm{B}$ cells in blood compared with conventional methods. Journal of Clinical Pathology, 37:1343.

HUMPHREY, J.H. and GRENNAN, D. (1982) Isolation and properties of spleen follicular dendritic cells. Advanced Experimental Medicine and Biology, 149:823.

HUSTED, S. (1975) Immobilizing and cytotoxic sperm antibodies in serum and seminal plasma and their relation to other sperm antibodies. Acta Pathologica et Microbiologica Scandinavica (c), 83:338.

IWATANI, Y., ROW, V.V. and VOLPE, R. (1985) What prevents autoimmunity? Lancet, 2:839.

JAGER, S., KREMER, J. and VAN SLOCHTEREN-DRAAISMA, T. (1978) A simple method of screening for antisperm antibodies in the human male. Detection of spermatozoal surface IgG with the direct mixed antiglobulin reaction carried out on untreated fresh human semen. International Journal of Fertility, 23:12.

JAGER, S., KREMER, J., KUIKEN, J.and VAN SCLOCHTEREN-DRAAISMA, T. (1980) Immunoglobulin class of antispermatozoal antibodies from infertile men and inhibition of in vitro sperm penetration into cervical mucus. International Journal of Andrology, 3:1.

JAMES, K. and HARGREAVE, T.B. (1984) Immunosuppression by seminal plasma and its possible clinical significance. Immunology Today, 5:357.

JAMES, K. and RITCHIE, A.W.S. (1984) Do natural killer cells regulate B-cell activity? Immunology Today, 5:193.

JARROW, J.P., BUDIN, R.E., DYM, M., ZIRKIN, B.R., NOREN, S. and MARSHALL, F.F. (1985) Quantitative pathologic changes in the human testis after vasectomy. The New England Journal of Medicine, 14:1252.

JASANI, B., WYNFORD THOMAS, D. and WILLIAMS, E.D. (1981), Use of monoclonal antihapten antibodies for immunolocalisation of tissue antigen. Journal of Clinical Pathology, 34:1000.

JENKINS, A.D., TURNER, T.T. and HOWARDS, S.S. (1978) Physiology of the Male Reproductive System. Urologic Clinics of North America, 5:437.

JOHNSON, M.H. (1973) Physiological mechanisms for the immunological isolation of spermatozoa. Advances in Reproductive Physiology, 4: 279. 
JONES, R.C. (1977) The nature of the barrier to autoimmunity in the excurrent ducts of the mammalian testes. In: B. Boettcher (ed.) Immunological Influences on Human Fertility, p.67. New York: Academic Press.

JONES, W.R. (1980) Immunologic infertility: fact or fiction? Fertility and Sterility, 33(6):577.

KAMOUN, M., MARTIN, P.J., HANSON, J.A., BROWN, M.A., SIADAK, A.W. and NOWINSKI, R.C. (1981) Identification of human T lymphocyte surface protein associated with the E rosette receptor. Journal of Experimental Medicine, ?:153.

KAMPERDIJK, W.E.A., RAAYMARKERS, E.M., de LEEUW, J.H.S. and HOEFSMIT, E.C.M. (1978) Lymph node macrophages and reticulum cells in the immune response. I. The primary response to paratyphoid vaccine. Cell Tissue Research, 1921.

KAMPS, W.A. and COOPER, M.D. (1982) Microenvironmental studies of pre $B$ and $B$ cell development in human and mouse fetuses. Journal of Immunology, 129:526.

KAPPLER, J.W. and MARACK, P.C. (1976) Helper T-cells recognise antigen and macrophage surface components simultaneously. Nature, 262:797.

KASAHARA, T., HOOKS, J.J., DOUGHERTY, S.F. and OPPENHEIM, J.J. (1983) Interleukin 2-mediated immune interferon (IFN-8) production by human $\mathrm{T}$ cells and $\mathrm{T}$ cell subsets. Journal of Immunology, 130:1784.

KATSH, S. (1959) Infertility in female guinea pigs induced by injection of homologous sperm. American Journal of Obstetrics and Gynecology, 78:276.

KAUFMANN, S.H.E. (1984) Acquired resistance to facultative intracellular bacteria. Relationship between persistence, crossreactivity on the $T$ cell level and capacity to stimulate cellular immunity of different listeria strains. Journal of Infection and Immunology, 45:234.

KEARNEY, J.F., COOPER, M.D., KLEIN, J., ABNEY, E.R., PARKHOUSE, R.M.E. and LAWTON, A.R. (1977) Ontogeny of $\mathrm{Ia}$ and IgD on IgM bearing 1ymphocytes in mice. Journal of Experimental Medicine, 146:1297.

KERR, J.B. and de KRETSER, D.M. (1981) The cytology of the human testis. In: H. Burger and D.M. de Kretser (eds.), Comprehensive Endocrinology: The Testis, pp.141-179, New York: Raven.

KHAN, S.A., SODER, O. and SYED, V. (1987) The rat testis produces large amounts of an interleukin-1 like factor. International Journal of Andrology. In press. 
KLEIN, J. (1982) The organs of immune response. In: J. Klein (ed.) Immunology: The Science of Self-Non Self Discrimination, Ch. 4, pp.54-91. New York: A. Wiley Interscience Publication.

KOHLER, G. and MILSTEIN, C. (1976) Derivation of specific antibody-producing tissue culture and tumor lines by cell fusion. European Journal of Immunology, 6:511.

KONDA, S., STOCKERT, E. and SMITH, R.T. (1973) Immunologic properties of mouse thymus cells: membrane antigen patterns. Cel1 Immunology, 7:275.

KORMANO, M. (1967) Dye permeability and alkaline phosphatase activity of testicular capillaries in the post-natal rat. Histochemistry, 9:327.

KOSKIMIES, A.I., KORMANO, M. and ALFTHAU, 0. (1973) Proteins of the seminiferous tubule fluid in man - evidence for a blood-testis barrier. Journal of Reproduction and Fertility, 32: 79 .

KOTANI, M., OKADA, K., FUJII, H., ISUCHIYA, H., MATSUMO, K. , EKINO, S. and FUKADA, S. (1977) Lymph macrophages enter the germinal centre of lymph nodes of guinea pigs. Acta Anatomica, 99:391.

KOTANI, M., EZAKI, T., EKINO, S., MATSUMO, K., FUJII, H. and NAWA, Y. (1982) Lymph macrophages enter the germinal centres of regional 1ymph nodes. Advances in Experimental Medicine and Biology, 149:837.

KRIEGER, J.N. (1984) Prostatitis syndromes: Pathophysiology, differential diagnosis and treatment. Sexually Transmitted Diseases, 11:100.

LAMPSON, L.A. and LEVY, R. (1980) Two populations of Ia-like molecules on a human B cell line. Journal of Immunology, $125: 393$.

LANDSTEINER, K. (1899) Zur kenntnis der specifisch auf blutkozperchen wirkenden sera. Zbl. Bakt., 25:546.

LANIER, L.L., LE, A.M., PHILLIPS, J.H., WARNER, N.L. and BABCOCK, G.F. (1983) Subpopulations of human natural killer cells defined by expression of Leu 7 (HNK-1) and Leu 11 (NK-P-15) antigens. Journal of Immunology, 131:1789.

LEDBETTER, J.A., EVANS, R.L., LIPINSKI, M., CUNNINGHAM-RUNDLES, C., GOOD, R.A. and HERZENBERG, L.A. (1981) Evolutionary conservation of surface molecules that distinguish T lymphocyte helper/inducer and cytotoxic/suppressor subpopulations in mouse and man. Journal of Experimental Medicine, 153:310.

LEYDIG, F. (1850) Zur anatomie der mannlichen geschtsorgane und analdrusen der saugetiere. Z. Wiss. Zool., 2:1. 
LILLIE, R.D. (1965) Histopathological technique and practical histochemistry, p.137. New York: McGraw-Hill Book Company.

LONDON, S.N., HANEY, A.F., WEINBERG, J.B. (1985) Macrophages and infertility: enhancement of human macrophage-mediated sperm killing by antibodies. Fertility and Sterility, 43:274.

LOWY, A., DREBIN, J.A., MONROE, J.G., GRANSTEIN, R.D. and GREENE, M.I. (1984) Genetically restricted antigen presentation for immunological tolerance and suppression. Nature, 308:373.

McGOWAN, M.P., BURGER, H.G., BAKER, H.W.G., de KESTER, D.M. and KOVACS, G. (1981) The incidence of non specific infection in the semen in fertile and subfertile males. International Journal of Andrology, 4:657.

MacKANESS, G.B. (1964) The immunological basis of acquired cellular resistance. Journal of Experimental Medicine, 120:105.

McLAREN, A. (1964) Immunological control of fertility in female mice. Nature, 201:582.

McNEAL, J.E. (1981) The zonal anatomy of the prostate. Prostate, $1: 35$.

MANCINI, R.E., ANDRADA, J.A., SARCENI, D., BACHMAN, A.E., LAVIERI, J.C. et al. (1965) Immunological and testicular response in man sensitized with human testicular homogenate. Journal of Clinical and Endocrinological Metabolism, 25:859.

MANCINI, R.E. (1974) Immunologic and testicular response to a damage induced in the contralateral gland. In: R.E. Mancini and L. Martini, Male Fertility and Sterility, pp.271-300. New York: Academic Press.

MANN, T. and MANN, C.L. (1981) Male Reproductive Function and Semen. New York: Springer-Verlag.

MASON, T.E., PHIFER, R.F., SPICER, S.S. and SWALOW, R.A. (1969) An immunoglobulin-enzyme bridge method for localizing tissue antigens. Journal of Histochemistry and Cytochemistry, 17:563.

MASON, D.Y. and SAMMONS, R.E. (1978) Alkaline phosphatase and peroxidase for double immuno-enzymatic labelling of cellular constituents. Journal of Clinical Pathology, 31:454.

MASON, D.Y. and SAMMONS, R.E. (1979) The labelled antigen method of immuno-enzymatic staining. Journal of Histochemistry and Cytochemistry, 27:832. 
MASON, D.Y., NAIEM, M., ABDULAZIZ, Z., NASH, J.R.G., GATTER, K.C. and STEIN, H. (1982) Immunohistological applications of monoclonal antibodies. In: A.J. McMichael and J.W. Fabre (eds.), Monoclonal Antibodies in Clinical Medicine, pp.585-635. London: Academic Press.

MASON, D.Y., ERBER, W.N., FALINI, B.I., STEIN, H. and GATTER, K.C. (1986) Immuno-enzymatic labelling of haematological samples with monoclonal antibodies. In: P.C.L. Beverley (ed.), Methods in Hematology. Monoclonal Antibodies, pp.145-181. London: Churchill Livingstone.

MASTROGIACOMO, I., ZANCHETTA, R., GRAZIOTTI, P., BETTERLE, C., SCRUFARI, P. and LEMBO, A. (1982) Immunological and clinical study in patients after spermatic cord torsion. Andrologia, $14: 25$.

MATHUR, S. (1985) Immune and immunogenetic mechanisms in infertility. Contributions to Gynecology and Obstetrics, $14: 138$.

MATTHEWS, C.D., ELMSLIE, R.G., CLAPP, K.H. and SVIGOS, J.M. The frequency of genital mycoplasma infection in human fertility. Fertility and Sterility, 26:988.

MENGE, A.C. (1971) Antiserum inhibition of rabbit spermatozoal adherence to ova. Proceedings of the Society of Experimental Biological Medicine, 138:98.

METCHNIKOFF, E. (1899) Etudes sur la resorption des cellules. Annales de 1'Institut Pasteur, 13:737.

METLANIKOFF, S. (1900) Etudes sur la spermotoscine. Annales de 1'Institut Pasteur, 14:577.

MEUER, S.C., HUSSEY, R.E., PENTA, A.C., FITZGERALD, K.A., STADLER, B.M., SCHLOSSMAN, S.F. and REINHERZ, E.L. (1982a) Cellular origin of interleukin 2 (1L2) in man - evidence for stimulus restricted $1 \mathrm{~L} 2$ production by $\mathrm{T}^{+}$and $\mathrm{T}^{+} \mathrm{T}$ 1ymphocytes. Journal of Immunology, 129:1076.

MEUER, S.C., HUSSEY, R.E., HODGSON, J.C., HERCEND, T. and SCHLOSSMAN, S.F. (1982b) Surface structures involved in target recognition by human T-1ymphocytes. Science, 218:471.

MILLER, S.C., BOWMAN, B.M. and ROBERTS, L.K. (1984) Identification and characterization of mononuclear phagocytes isolated from rat testicular interstitial tissues. Journal of Leukocyte Biology, $36: 679$.

MIZEL, S.B. (1982) Interleukin 1 and $\mathrm{T}$ cell activation. Immunology Review, 63:51. 
MOBERG, P.J., ENEROTH, P., LJUNG, A. and NORD, C. (1980) Bacteria1 flora in semen before and after doxycline treatment in infertile couples. International Journal of Andrology, 3:45.

MOIR, D.J., GHOSH, A.K., ABDULAZIZ, Z., KNIGHT, P.M. and MARSON, D.Y. (1983) Immunoenzymatic staining of haematological samples with monoclonal antibodies. British Journal of Haematology, 55:395.

MOORE, M.A.S. and OWEN, J.J.T. (1967) Experimental studies on the development of the thymus. Journal of Experimental Medicine, $126: 715$.

MORETTA, L., WEBB, S.R., GROSSI, C.E., LYDYARD, P.M. and COOPER, M.D. (1977) Functional analysis of two human T cell subpopulations: help and suppression of B cell responses by $T$ cells bearing receptors for IgM (TM) or IgG (TG). Journal of Experimental Medicine, 146:184.

MORETTA, L., MINGARI, M.C., SEKALY, P.R., MORETTA, A., CHAPIUS, B. and CEROTTINI, J.C. (1981) Surface markers of cloned human T cells with various cytolytic activities. Journal of Experimental Medicine, 154:569.

MORTON, R.S. (1968) White cell counts in human semen. Their use in the diagnosis of prostatitis with reference to uveitis. British Journal of Venereal Diseases, 44:72.

MOWAT, A.M. and FERGUSON, A. (1981) Induction and expression of mucosal cell-mediated immunity. In: F.J. Bourne (ed.), The Mucosal Immune System, pp.107-129. The Hague/Boston/London: Mertinus Nijloff.

MOXTER Von (1900), Ueber ein specifisches immunserum gegen spermatozoen. Deutsche Medizinische Wochenschrift, 26:61.

MURRAY, H.W., RUBIN, B.Y. and ROTHERMAL, C.-D. (1983) Killing of intracellular Leishmania donovani by lymphokine-stimulated human mononuclear phagocytosis: evidence that interferon-Y is the activating lymphokine. Journal of Clinical Investigation, $72: 1506$.

NADJI, M. (1980) The potential value of immunoperoxidase techniques in diagnostic cytology. Acta Cytologica, 24(5):442.

NAESSENS, A., FOULON, W., DEBRUCKER, P., DEVROEY, P. and LAWERS, S. (1986) Recovery of micro-organisms in semen and relationship to semen evaluation. Fertility and Sterility, 45:101-105.

NAGARKATTI, P.S. and RAO, S.S. (1976) Cell-mediated immunity to homologous spermatozoa following vasectomy in the human male. Clinical and Experimental Immunology, 26:239. 
NAHOWM, C.R.D. and CARDOZO, D. (1980) Staining for volumetric count of leukocytes in semen and prostate-vesicular fluid. Fertility and Sterility, 34:68.

NAKANE, P.K. and PIERCE, G.B. (1966) Enzyme labelled antibodies: preparation and application for the localization of antigens. Journal of Histochemistry and Cytochemistry, 14:929.

NAKANE, P.K. and KAWAOI, A. (1974) Peroxidase labelled antibody. A new method of conjugation. Journal of Histochemistry and Cytochemistry, 22:1084.

NATHAN, C.F., MURRAY, H.W., WIEBE, M.W. and RUBIN, B.Y. (1983) Identification of interferon as the lymphokine that activates human macrophage oxidative metabolism and antimicrobial activity. Journal of Experimental Medicine, 158:670.

NELSON, D.A. and DAVEY, F.R. (1977) Leukocyte peroxidase. In: W.J. Williams (ed.) Hematology, ed. 2, Ch. A.20, p.1627. New York: McGraw Hill Book Company.

NISTAL, M., ABAURREA, M.A. and PANIAGUA, R. (1982) Morphological and histometric study on the human Sertoli cell from birth to the onset of puberty. Journal of Anatomy, 14:351.

OLIPHANT, G., CABOT, C., ROSS, P. and MARTA, J. (1984) Control of humoral immune system within the rabbit oviduct. Biology of Reproduction, 31:205.

O'REILLY, R.J., LEE, L. and WELCH, B.G. (1976) Secretory IgA antibody response to Neisseria gonorrhoeae in the genital secretions of infected females. Journal of Infectious Diseases, 133: 113 .

OSMOND, D.G. (1980) Production and differentiation of B lymphocytes in the bone marrow. In: J.R. Battisto and K.L. Knight (eds.), Immunoglobulin Genes and B Cell Differentiation, p.135. North Holland/New York: Elsevier.

OVERSTREET, J.W. and COOPER, G.W. (1978) Sperm transport in the reproductive tract of the female rabbit. II. The sustained phase of transport. Biology of Reproduction, 19:115.

OVERSTREET, J.W. (1986) Human sperm function = acquisition in the male and expression in the female. In: R.J. Santen and R.W. Swendloff (eds.), Male Reproductive Function, p.29. New York: Marcel Dekker Inc.

OWEN, J.T.T. and RITTER, M.A. (1969) Tissue interaction in the development of thymus lymphocytes. Journal of Experimental Medicine, 129, 431-437.

OWEN, J.T.T. and RAFFE, M.C. (1970) Studies on the differentiation of thymus derived lymphocytes. Journal of Experimental

Medicine, 132:216. 
PERUSSIA, B., FANNING, V. and TRINCHIERI, G. (1983) A human NK and $\mathrm{K}$ cell subset shares with cytotoxic T-cells expression of the antigen recognized by antibody OKT8. Journal of Immunology, $131: 223$.

PICHLER, W.J., LUM, L. and BRODER, S. (1978) FC-receptors on human $\mathrm{T}$ 1ymphocytes. I. Transition of $\mathrm{Tg}$ to Tu cells. Journal of Immunology, 121:1540.

POOL, W.C., BUIJS, R.M., SWAAB, D.F., BOER, G.J. and Van LEEUWEN, F.W. (1983) On the way to a specific immunocytochemical localization. In: A.C. Cuello (ed.), Immunocytochemistry, pp.2-45. IBRO.

POPPEMA, S., BHAN, A.K., REINHERZ, E.L., MCCLUSKEY, R.T. and SCHLOSSMAN, S.F. (1981) Distribution of $\mathrm{T}$ cell subsets in human lymph nodes. Journal of Experimental Medicine, 153:30.

QUESEDA, E.M., DUKES, G.D., DEEN, G.H. and FRANKLIN, R.R.

Genital infection and sperm agglutinating antibodies in infertile men. Journal of Urology, 19:106.

RAO, S.S. and SADRI, K.K. (1959) Immunological studies with human semen and cervical mucus. Proceedings of the Sixth International Conference on Planned Parenthood, New Delhi, p.313.

REID, B.L. and CLELAND, K.W. (1957) The structure and function of the epididymis. I. The histology of the rat epididymis. Australian Journal of Zoology, $5: 223$.

REINHERZ, E.L. and SCHLOSSMAN, S.F. (1979) Con A inducible suppression of MLC: evidence for mediation by the $\mathrm{TH}_{2}+\mathrm{T}$ cell subset in man. Journal of Immunology, 122:1335.

REINHERZ, E.L., KUNG, P.C., GOLDSTEIN, G. and SCHLOSSMAN, S.F. (1979) A separation of functional subsets of human $T$ cells by a monoclonal antibody. Proceedings of the National Academy of Science USA, 76:4061.

REINHERZ, E.L. and SCHLOSSMAN, S.F. (1980a) The differentiation and function of human T-1ymphocytes. Cell, 19:821.

REINHERZ, E.L. and S.F. SCHLOSSMAN (1980b) Regulation of the immune response: inducer and suppressor $\mathrm{T}$ lymphocyte subsets in man. New England Journal of Medicine, 303:370.

REINHERZ, E.L., HUSSEY, R.E. and SCHLOSSMAN, S.F. (1980a) A monoclonal antibody blocking human T-cell function. European Journal of Immunology, 10:758. 
REINHERZ, E.L., NORIMOTO, C., PENTA, A.C. and SCHLOSSMAN, S.F. (1980b) Regulation of B cell immunoglobulin secretion by functional subsets of T-1ymphocytes in man. European Journal of Immunology, 10, 570 .

REINHERZ, E.L., KUNG, P.C., GOLDSTEIN, G., LEVEY, R.H. and SCHLOSSMAN, S.F. (1980c) A monoclonal antibody reactive with the human cytotoxic/suppressor $\mathrm{T}$ cell subset previously defined by a hetero-antiserum termed $\mathrm{TH}_{2}$. Journal of Immunology, $124: 1301$.

REINHERZ, E.L., KUNG, P.C., GOLDSTEIN, G., LEVEY, R.H. and SCHLOSSMAN, S.F. (1980d) Discrete stages of human introthymic differentiation: analysis of normal thymocytes and leukemic lymphoblasts of $\mathrm{T}$ lineage. Proceedings of the National Academy of Science USA, 77:1588.

RICHMAN, L.K., CHILLER, J.M., BROWN, W.R., HANSON, D.G. and VAZ, N.M. (1978) Enterically induced immunological tolerance. I. Induction of suppressor $\mathrm{T}$ lymphocytes by intragastric administration of soluble proteins. Journal of Immunology, $121: 2429$.

RITCHIE, A.W.S., JAMES, K. and MICKLEM, H.S. (1983) The distribution and possible significance of cells identified in human lymphoid tissue by monoclonal antibody HNK-1. Clinical and Experimental Immunology, 51:439.

RITCHIE, A.W.S., HARGREAVE, T.B., JAMES, K. and CHISHOLM, G.D. (1984a) Intra-epithelial lymphocytes in the normal epididymis. A mechanism for tolerance to sperm auto-antigens? British Journal of Urology, 56:79.

RITCHIE, A.W.S., JAMES, K., MICKLEM, S. and CHISHOLM, G.D. (1984b) Lymphocyte subsets in renal carcinoma - a sequential study using monoclonal antibodies. British Journal of Urology, 56:140.

RIVA, A. (1982) The ampulla ductus deferentis in man, as viewed by SEM and TEM. Archives of Andrology, 8:157-164.

ROBERTSON, M. (1985) T-cell receptor, the present state of recognition. Nature, 317:768.

ROITT, I.M., BROSTOFF, J. and MALE, D.K. (1985) Cells involved in the immune response. In: I.M. Rott, J. Brostoff and D.K. Male (eds.) Immunology, pp.2; 8-15. Edinburgh/London/New York: Churchill Livingstone.

ROMRELL, L.J. and O'RAND, M.G. (1978) Capping and ultrastructural localization of sperm surface isoantigens during spermatogenesis. Developmental Biology, 63:76.

ROOSEN-RUNGE, E.C. and HOLSTEIN, A.F. (1978) The human rete testis. Cell Tissue Research, 189:409. 
ROSS, L.S. (1983) Diagnosis and treatment of infertile men: a clinical perspective. The Journal of Urology, 130: 847.

ROTH, J., BENDAYAN, M. and ORCI, L. (1978) Ultrastructural localisation of intracellular antigens by the use of protein A gold complex. Journal of Histochemistry and Cytochemistry, $26: 1074$.

RUMKE, P., Van AMSTEL, N., MESSER, E.N. and BEXEMER, P.D. (1974): Prognosis of fertility of men with sperm agglutinins in the serum. Fertility and Sterility, 25:393.

RUMKE, P.H. (1954) The presence of sperm antibodies within the serum of two patients with oligozoospermia. Vox Sanguinis, 4:135.

RUMKE, P. and HELLINGER, G. (1959) Autoantibodies against spermatozoa in sterile men. American Journal of Clinical Pathology, 32:357.

RUMKE, P. and TITUS, M. (1970) Spermagglutinin formation in male rats by subcutaneous injected syngenic epididymal spermatozoa and by vasoligation or vasectomy. Journal of Reproduction and Fertility, 21:69.

RUMKE, P. (1982) Detection of autoantibodies on motile spermatozoa and mechanisms of autoimmune infertility in man. In:

S. Sheilman, F. Dondero and M. Nictotra (eds.), Immunological Factors in Human Reproduction, pp.70-75. London: Academic Press.

SALING, P.M., RAINES, L.M. and O'RAND, M.G. (1983) Monoclonal antibody against mouse sperm blocks: a specific event in the fertilization process. Journal of Experimental Biology, $227: 481$.

SAMUEL, T. and ROSE, N.R. (1980) The lessons of vasectomy - a review. Journal of Clinical Laboratory Immunology, 3:77.

SARKAR, S. (1974) Carbohydrate antigens of human sperm and autoimmune induction of infertility. Journal of Reproductive Medicine, 13:93.

SCHER, M.G., UNANUE, E.R. and BELLEN, D.I. (1982) Regulation of macrophage populations. III. The immunologic induction of exudates rich in Ia-positive macrophages is a radiosensitive process. Journal of Immunology, 138:447.

SCHLOSSMAN, S.F. and REINHERZ, E.L. (1984) Human T-cell subsets in health and disease. Springer Seminars in Immunopathology, 7:9.

SCHULZE, C. (1984) Sertoli cells and Leydig cells in man. Advances in Anatomy, Embryology and Cell Biology, 88:7. 
SEKI, M. and METTLER, L. (1982) Influence of spermatozoal antibodies in the reproduction of mice. American Journal of Reproductive Immunology, 2:225.

SELBY, W.S., JANOSSY, G., GOLDSTEIN, G. and JEWELL, J.P.

T-1ymphocyte subsets in human intestinal mucosa: the distribution and relationship to MHC-derived antigens. Clinical and Experimental Immunology, 44:453.

SERTOLI, E. (1865) Dell'esistenza di particulari cellule ramificate nei canalicoli seminiferi dell'testiculo umano. Morgagni, 7:31.

SETCHELL, B.P., LAURIE, M.S. and FRITZ, I.B. (1980) Development of the function of the blood-testis barrier in rats and mice. In: E. Steinberger and A. Steinberger (eds.), Testicular Development, Structure and Function, pp. 65-69. New York: Raven Press.

SHIVERS, C.A. and DUNBAR, (1977) Autoantibodies to Zona pellucida: a possible cause for infertility in women. Science, 197: 1082 .

SHORTLIFFE, L.M.D., WEHNER, N. and STAMEY, T.A. (1981) The detection of a local prostatic immunologic response to bacterial prostatitis. Journal of Urology, 125:509.

SHULMAN, S. (1975) Reproduction and Antibody Response. Cleveland: CRC Press.

SHULMAN, S. and ORSINI, F. (1970) The antigens of seminal vesicle and seminal plasma. Fertility and Sterility, 21:794.

SMITH, K.A., GILBRIDE, K.J. and FAVATA, M.F. (1980) Lymphocyte activating factor promotes $\mathrm{T}$ cell growth factor production by cloned murine 1ymphoma cells. Nature (London), 287:353.

STASHENKO, P., NADLER, L.M., HARDY, R. and SCHLOSSMAN, S.F. (1980) Characterization of a human B 1ymphocyte specific antigen. Journal of Immunology, 125:1678.

STEEG, R.S., MOORE, R.N., JOHNSON, J.M. and OPPENHEIM, J.J. (1982) Regulation of murine macrophage Ia antigen expression by a lymphokine with immune interferon activity. Journal of Experimental Medicine, 156:1780.

STEINMAN, R.M. (1981) Dendritic Cells Transplantation. Baltimore, 31,151 .

STERNBERGER, L.A., HARDY, P.H., Jr., CUCULIS, J.J. and MEYER, H.G. (1970) The unlabelled antibody enzyme method for immunohistochemistry. Preparation and properties of soluble antigenantibody complex (horseradish peroxidase) and its use in identification of spirochetes. Journal of Histochemistry and Cytochemistry, 22:315. 
STINGL, G., KATZ, S.I., CLEMENT, L., GREEN, I. and SHEVACH, E.M. (1978) Immunologic functions of Ia-bearing epidermal Langerhans cells. Journal of Immunology, 121:2005.

STRAUS, W. (1982) Imidazol increases the sensitivity of the cytochemical reaction for peroxidase with diaminobenzidine at neutral pH. Journal of Histochemistry and Cytochemistry, $30: 491$.

SWENSON, C.E., TOTH, A., TOTH, C., WOLFGRUBEN, L. and O'LEARY, W.M. (1980) A symptomatic bacteriospermia in infertile men. Andrologia, 12:7.

TAYLOR-ROBINSON, D., MARTIN BOURGON, C., WATANABE, T. and ADDEY, J.P. (1971) Isolation of T mycoplasmas from dogs and squirrel monkeys: biological and serological comparison with those isolated from man and cattle. Journal of General Microbiology, 68:97.

THESTRUP-PEDERSEN, K., HUSTED, S. and HJORT, T. (1976) Lymphocyte-transformation test with spermatozoal antigens in men from infertile couples. I. A methodological study of different spermatozoal preparations as antigens. International Journal of Fertility, 21:218.

THOMAS, B.J., EVANS, R.T., HUTCHINSON, G.R. and TAYLOR ROBINSON, D. (1977) Early detection of chlamydial inclusions combining the use of cycloheximide-treated McCoy cells and immunofluorescence staining. Journal of Clinical Microbiology, $6: 285$.

THOMAS, Y., ROGOZINSKI, L., IRIGOYEN, O., FRIEDMAN, S.M., KING, P.C., GOLDSTEIN, G. and CHESS, L. (1981) Functional analysis of human $\mathrm{T}$ cell subsets defined by monoclonal antibodies. IV. Induction of suppressor cells within the $\mathrm{OKT}_{4}{ }^{+}$ populations. Journal of Experimental Medicine, 154:459.

TIMENS, W. and POPPEMA, S. (1985) Lymphocyte compartments in human spleen and non involved spleens in Hodgkin's disease. American Journal of Pathology, 120(3):443.

TUNG, K.S.K., OKADA, A., YANAGIMACHI, R. (1980) Sperm autoantigens and fertilization. I. Effects of antisperm antibodies on rouleaux formation, viability and acrosome reaction of guinea pig sperm. Biology of Reproduction, 23:877.

UCHIYAMA, T., BRODER, S. and WALDMANN, T.A. (1981) A monoclonal antibody (anti-Tac) reactive with activated and functionally mature human T-cells. I. Production of anti-Tac monoclonal antibody and distribution of $\mathrm{Tac}^{+}$cells. Journal of Immunology, $126: 1393$ : $^{\circ}$

UEHLING, D.T. (1971) Secretory IgA in seminal fluid. Fertility and Sterility, 22:769. 
ULSTEIN, M., CAPELL, P., HOLMES, K.K. and POULSEN, C.A. (1976) Non-symptomatic genital tract infection and male infertility. In: E.S.E. Hafez (ed.), Human Semen and Fertility Regulation in Men, pp.355-362. Mosby: St Louis.

UNANUE, E.R. (1981) The regulatory role of macrophages in antigenic stimulation. Part Two: Symbiotic relationship between 1ymphocytes and macrophages. Advances in Immunology, 31:1.

UPADHYAYA, M., HIBBARD, B.M. and WALKER, S.M. (1984) The effect of urealyticum on semen characteristics. Fertility and Sterility, $41: 304$.

URRY, R.L. (1981) Pathophysiological principles of male infertility. Urology Clinic, North Am., 8:3.

Van VOORHIS, N.C., HAIR, L.S., STEINMAN, R.M. and KAPLAN, G. (1982) Human dendritic cells. Enrichment and characterization from peripheral blood. Journal of Experimental Medicine, 155:1172, 1187.

VEERMAN, A.J.P. (1974) On the interdigitating cells in the thymus-dependent area of rat spleen: a relation between the mononuclear phagocyte systems and T-1ymphocytes. Cell Tissue Research, 148:247.

VENDRELY, E. (1981) Histology of the epididymis in the human adult. Progress in Reproductive Biology and Medicine, 8:21.

VITALE, R., FAWCETT, D.W. and DYM, M. (1973) The normal developmental blood testis barrier and the effects of clomiphene and oestrogen treatment. Anatomical Record, 176:333.

WANG, Y.F. and HOLSTEIN, A.F. (1983) Intraepithelial 1ymphocytes and macrophages in the human epididymis. Cell Tissue Research, $233: 517$.

WAHAB, Z.A. and WRIGHT, G.L. (1985) Monoclonal antibody (anti-leu 7) directed against natural killer cells reacts with normal, benign and malignant prostate tissues. International Journal of Cancer, 36:677.

WEIL, A.J. (1960) Immunological differentiation of epididymal and seminal spermatozoa of the rabbit. Science, 131:1040.

WEST, W.H., BROOZER, R.B. and HERBERMAN, R.B. (1978) Low affinity $\mathrm{E}$ rosette formation by the human $\mathrm{K}$ cell. Journal of Immunology, 120:90.

WILSON, L. (1954) Sperm agglutinins in human semen and blood. Proceedings of the Society of Experimental Biological Medicine, $85: 652$. 
WITKIN, S.S., ZELIKOVSKY, G., GOOD, R.A. and DAY, N. (1981) Demonstration of $11 \mathrm{~s} I g A$ antibody to spermatozoa in human seminal fluid. Clinical and Experimental Immunology, 44:368.

WITKIN, S.S. and TOTH, A. (1983) Relationship between genital tract infections, sperm antibodies in seminal fluid and infertility. Fertility and Sterility, 40:805.

WOOD, G.S., WARNER, N.L. and WARNKE, R.A. (1983) Anti leu 3/?T4 antibodies react with cells of monocyte/macrophage and Langerhans lineage. Journal of Immunology, 131:212.

YACHIE, A., MIYAWAKI, T., YOKOI, T., NAGAOKI, T. and TANIGUCHI, N. (1982) Ia positive cells generated by PWM-stimulation within $\mathrm{OKT}_{4}{ }^{+}$subset interact with $\mathrm{OKT}^{+}$cells for inducing active suppression on B cell differentiation in vitro. Journal of Immunology, 129:103.

YANAGIMACHI, R., OKADA, A. and TUNG, K.S.K. (1981) Sperm auto-antigens and fertilization. II. Effects of anti guinea pig serum antibodies on sperm ovum interactions. Biology of Reproduction, 24:512.

YOUNG, H., TUACH, S. and BAIN, S.S.R. (1981) Incidence of Ureaplasma urealyticum infection in women attending a clinic for sexually transmitted diseases. Journal of Infection, 3:258.

YOUNG, H., SARAFIAN, S.K., HARRIS, A.B. and McMILLAN, A. (1983) Non-cultural detection of Neisseria gonorrhoeae in cervical and vaginal washings. Journal of Medical Microbiology, 16:183.

ZIEGLER, K. and UNANUE, E.R. (1981) Identification of a macrophage antigen-processing event required for I region - restricted antigen presentation to $\mathrm{T}$ lymphocytes. Journal of Immunology, $127: 1869$. 
APPENDICES 
Preparation of buffered saline:

1. Tris Buffered Saline (TBS)

This was prepared using $0.85 \mathrm{NaCl}$ buffered with Tris-HC1, $\mathrm{pH} 7.6,0.01 \mathrm{M}$.

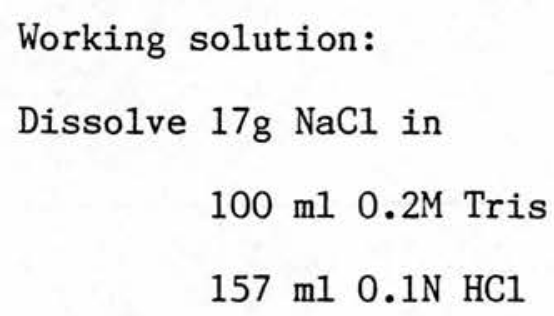

2. Phosphate Buffered Saline (PBS)

$$
\begin{array}{ll}
0.145 \mathrm{M} \mathrm{NaCl}, 0.018 \mathrm{M} \mathrm{K}_{2} \mathrm{HPO}_{4}, & \\
0.01 \mathrm{M} \mathrm{KH}_{2} \mathrm{PO}_{4} & \mathrm{pH} 7.4
\end{array}
$$

$25 \times$ stock solution

Dissolve in 1 litre of distilled water:

$$
\begin{aligned}
& \mathrm{NaCl} \\
& \mathrm{K}_{2} \mathrm{HPO}_{4} \\
& \mathrm{KH}_{2} \mathrm{PO}_{4}
\end{aligned}
$$

$1 \mathrm{x}$ working solution

To 960 distilled water, add $40 \mathrm{ml} 25 \mathrm{x}$ stock solution or to $1920 \mathrm{ml}$ distilled water, add $80 \mathrm{ml} 25 \mathrm{x}$ stock. 
APPENDIX B

TABLE 1:

T-LYMPHOCYTE SUBSETS WITHIN THE EPITHELIUM OF THE EPIDIDYMUS (mean counts \pm S.D. per 100 epithelial cells)

\begin{tabular}{|c|c|c|c|c|}
\hline Donor & $\begin{array}{l}\text { Leu } 4^{+} \text {cells } \\
\text { (A11 T-cells) }\end{array}$ & $\begin{array}{c}\text { Leu } 2 \mathrm{a}^{+} \text {cells } \\
\text { ( } \mathrm{T} \text { suppressor } / \\
\text { cytotoxic) }\end{array}$ & $\begin{array}{c}\text { Leu } 3 \mathrm{a}^{+} \text {cells } \\
\text { ( } \mathrm{T} \mathrm{helper/} \\
\text { inducer) }\end{array}$ & $\begin{array}{l}\text { Leu } 2 a / \\
\text { Leu } 3 a\end{array}$ \\
\hline
\end{tabular}

A - HEAD

$\begin{array}{rrrrr}1 & 16.49 \pm 3.72 & 13.94 \pm 4.49 & 3.55 \pm 2.04 & 3.92 \\ 2 & 13.58 \pm 2.81 & 11.05 \pm 3.85 & 3.15 \pm 1.51 & 3.50 \\ 3 & 14.12 \pm 1.83 & 11.08 \pm 2.79 & 2.73 \pm 1.35 & 4.05 \\ 4 & 9.20 \pm 2.33 & 8.60 \pm 2.61 & 1.51 \pm 1.46 & 5.69 \\ 5 & 14.91 \pm 2.12 & 12.26 \pm 3.04 & 2.31 \pm 1.28 & 5.30 \\ 6 & 13.58 \pm 2.81 & 10.69 \pm 3.24 & 2.72 \pm 1.61 & 3.93 \\ 7 & 9.07 \pm 3.33 & 7.90 \pm 3.43 & 1.38 \pm 1.08 & 5.72 \\ 8 & 7.33 \pm 2.49 & 5.28 \pm 3.36 & 1.87 \pm 3.36 & 2.82\end{array}$

$\begin{array}{llll}\text { All donors } 12.30 \pm 3.30 & 10.10 \pm 2.70 & 2.40 \pm 2.70 & 4.45 \pm 1.17\end{array}$

\section{$\underline{B-B O D Y}$}

$\begin{array}{rrrrl}1 & 11.97 \pm 3.51 & 8.80 \pm 3.02 & 2.78 \pm 2.49 & 3.16 \\ 2 & 12.72 \pm 3.13 & 10.06 \pm 2.72 & 3.55 \pm 2.50 & 2.83 \\ 3 & 13.05 \pm 2.28 & 10.42 \pm 1.96 & 3.78 \pm 2.19 & 2.75 \\ 4 & 5.49 \pm 2.26 & 4.84 \pm 2.19 & 0.99 \pm 0.67 & 4.88 \\ 5 & 10.53 \pm 2.83 & 8.09 \pm 2.13 & 2.33 \pm 1.37 & 3.47 \\ 6 & 11.82 \pm 2.67 & 9.58 \pm 2.30 & 3.79 \pm 1.20 & 2.52 \\ 7 & 7.94 \pm 3.23 & 6.00 \pm 3.88 & 0.86 \pm 0.83 & 6.97 \\ 8 & 11.74 \pm 2.07 & 8.03 \pm 3.46 & 1.20 \pm 1.77 & 6.69 \\ & & & & \\ 11 & & & & \\ 1 & 10.70 \pm 2.60 & 8.20 \pm 2.0 & 2.40 \pm 1.30 & 4.15 \pm 1.8\end{array}$

\section{C - TAIL}

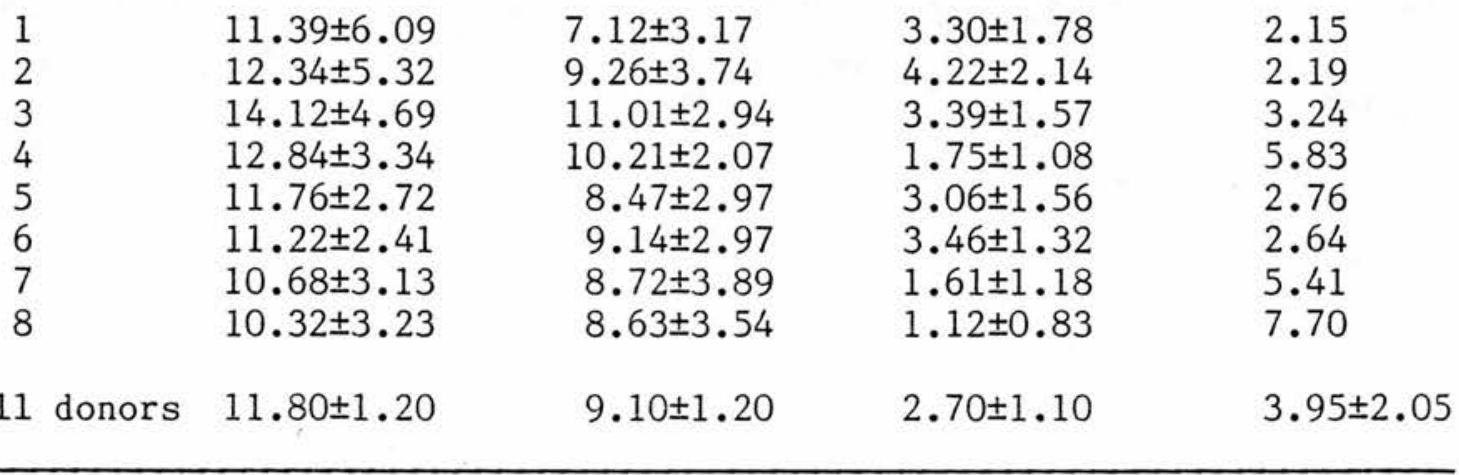


TABLE 2:

T-LYMPHOCYTE SUBSETS IN THE LAMINA PROPRIA OF THE EPIDIDYMIS (mean counts \pm S.D. per cross section of the epididymal duct)

\begin{tabular}{|c|c|c|c|c|}
\hline Donor & $\begin{array}{l}\text { Leu } 4^{+} \text {cells } \\
\text { (A11 T-cells) }\end{array}$ & $\begin{array}{l}\text { Leu } 2 \mathrm{a}^{+} \text {cells } \\
\text { ( } \mathrm{T} \text { suppressor/ } \\
\text { cytotoxic) }\end{array}$ & $\begin{array}{c}\text { Leu } 3 \mathrm{a}^{+} \text {cells } \\
\text { ( } \mathrm{T} \text { helper } / \\
\text { inducer) }\end{array}$ & $\begin{array}{l}\text { Leu } 2 a / \\
\text { Leu } 3 a\end{array}$ \\
\hline
\end{tabular}

\section{A - HEAD}

$\begin{array}{lllll}1 & 1.3 \pm 1.3 & 1.1 \pm 1.5 & 0.7 \pm 1.2 & 1.57 \\ 2 & 2.2 \pm 1.3 & 1.4 \pm 1.3 & 1.2 \pm 1.2 & 1.16 \\ 3 & 1.8 \pm 1.3 & 1.1 \pm 0.9 & 0.6 \pm 0.6 & 1.76 \\ 4 & 2.1 \pm 0.6 & 1.8 \pm 1.0 & 0.9 \pm 0.5 & 2.0 \\ 5 & 1.5 \pm 1.3 & 1.1 \pm 1.4 & 0.9 \pm 0.6 & 1.22 \\ 6 & 1.5 \pm 1.3 & 1.3 \pm 1.2 & 0.9 \pm 0.9 & 1.44 \\ 7 & 2.0 \pm 0.9 & 1.4 \pm 0.7 & 0.9 \pm 0.6 & 1.55 \\ 8 & 1.4 \pm 0.6 & 1.0 \pm 0.7 & 0.8 \pm 0.6 & 1.25\end{array}$

$\begin{array}{lllll}\text { Al1 donors } 1.7 \pm 0.3 & 1.3 \pm 0.3 & 0.9 \pm 0.20 & 1.49 \pm 0.28\end{array}$

\section{B - BODY}

$\begin{array}{lllll}1 & 2.9 \pm 1.9 & 1.7 \pm 1.3 & 1.9 \pm 0.9 & 0.89 \\ 2 & 2.7 \pm 2.5 & 1.3 \pm 1.3 & 1.1 \pm 1.0 & 1.18 \\ 3 & 3.0 \pm 1.4 & 1.8 \pm 0.9 & 1.1 \pm 0.9 & 1.63 \\ 4 & 1.7 \pm 0.7 & 1.3 \pm 0.6 & 1.2 \pm 0.7 & 1.08 \\ 5 & 1.9 \pm 0.9 & 1.4 \pm 0.9 & 1.3 \pm 0.9 & 1.07 \\ 6 & 2.1 \pm 1.5 & 1.3 \pm 1.3 & 0.6 \pm 0.9 & 2.16 \\ 7 & 1.6 \pm 0.7 & 1.4 \pm 0.7 & 0.7 \pm 0.5 & 2.00 \\ 8 & 2.7 \pm 1.2 & 2.4 \pm 1.1 & 0.7 \pm 0.6 & 3.42\end{array}$

$\begin{array}{lllll}\text { All donors } 2.3 \pm 0.6 & 1.6 \pm 0.4 & 1.1 \pm 0.4 & 1.68 \pm 0.84\end{array}$

\section{C - TAIL}

$\begin{array}{lllll}1 & 3.2 \pm 1.5 & 3.5 \pm 1.3 & 2.0 \pm 1.6 & 1.75 \\ 2 & 3.3 \pm 2.9 & 3.2 \pm 2.2 & 2.2 \pm 2.0 & 1.45 \\ 3 & 3.6 \pm 2.1 & 2.7 \pm 2.0 & 1.8 \pm 1.8 & 1.50 \\ 4 & 2.4 \pm 0.7 & 1.8 \pm 0.7 & 1.3 \pm 0.7 & 1.38 \\ 5 & 2.9 \pm 1.0 & 2.5 \pm 0.8 & 1.1 \pm 0.6 & 2.27 \\ 6 & 3.3 \pm 1.3 & 2.5 \pm 1.5 & 1.6 \pm 1.1 & 1.56 \\ 7 & 2.5 \pm 1.6 & 2.6 \pm 1.3 & 1.2 \pm 0.8 & 2.16 \\ 8 & 2.5 \pm 1.0 & 2.0 \pm 0.9 & 1.1 \pm 0.6 & 1.81\end{array}$

$\begin{array}{lllll}\text { All donors } 3.0 \pm 0.5 & 2.6 \pm 0.6 & 1.5 \pm 0.40 & 1.73 \pm 0.33\end{array}$


TABLE 3:

T-LYMPHOCYTE SUBSETS IN THE INTERSTITIAL CONNECTIVE TISSUES OF NORMAL HUMAN EPIDIDYMIS

(mean counts \pm S.D. per $0.8 \mathrm{~mm}^{2}$ interstitial tissues $\times 160$ )

\begin{tabular}{llcc} 
Leu $4^{+}$cells & $\begin{array}{l}\text { Leu } 2 \mathrm{a}^{+} \text {cells } \\
\text { (T suppressor/ } \\
\text { cytotoxic) }\end{array}$ & $\begin{array}{c}\text { Leu } 3 \mathrm{a}^{+} \text {cells } \\
\text { ( } \mathrm{T} \text { helper/ } \\
\text { inducer) }\end{array}$ \\
\hline
\end{tabular}

\section{A - HEAD}

$\begin{array}{rrrr}1 & 15.4 \pm 2.8 & 5.4 \pm 2.7 & 13.6 \pm 3.2 \\ 2 & 4.0 \pm 1.2 & 2.6 \pm 0.8 & 6.5 \pm 3.5 \\ 3 & 5.0 \pm 2.2 & 2.3 \pm 0.7 & 3.3 \pm 0.8 \\ 4 & 21.0 \pm 6.9 & 5.9 \pm 3.1 & 18.3 \pm 7.6 \\ 5 & 7.2 \pm 2.7 & 3.1 \pm 1.2 & 4.1 \pm 1.5 \\ 6 & 4.0 \pm 1.8 & 1.8 \pm 0.9 & 2.5 \pm 1.1 \\ 7 & 5.7 \pm 3.0 & 1.9 \pm 1.0 & 4.7 \pm 1.6 \\ 8 & 8.5 \pm 2.9 & 4.0 \pm 1.8 & 8.7 \pm 2.2 \\ & & & \\ \text { donors } & 6.5 \pm 4.1 & 3.4 \pm 1.6 & 7.7 \pm 5.5\end{array}$

B - BODY

$\begin{array}{lrrr}1 & 5.6 \pm 2.5 & 5.9 \pm 1.4 & 11.2 \pm 3.4 \\ 2 & 6.0 \pm 1.6 & 2.7 \pm 0.7 & 5.1 \pm 2.5 \\ 3 & 3.4 \pm 1.2 & 2.0 \pm 0.8 & 2.7 \pm 0.5 \\ 4 & 11.0 \pm 3.9 & 3.2 \pm 1.0 & 9.7 \pm 3.3 \\ 5 & 4.8 \pm 2.4 & 2.1 \pm 1.2 & 2.8 \pm 1.5 \\ 6 & 3.8 \pm 2.6 & 1.8 \pm 0.9 & 3.2 \pm 1.9 \\ 7 & 6.3 \pm 0.9 & 1.5 \pm 0.5 & 5.1 \pm 1.5 \\ 8 & 8.6 \pm 3.2 & 3.1 \pm 1.0 & 7.2 \pm 2.0 \\ & & & \\ \text { donors } & 7.4 \pm 4.1 & 2.8 \pm 1.4 & 5.9 \pm 3.2\end{array}$

$\underline{C}-$ TAIL

$\begin{array}{lrrr}1 & 9.8 \pm 2.4 & 4.0 \pm 2.1 & 7.8 \pm 1.5 \\ 2 & 8.8 \pm 1.9 & 2.9 \pm 0.9 & 7.8 \pm 2.0 \\ 3 & 8.0 \pm 3.5 & 3.3 \pm 2.5 & 3.9 \pm 2.4 \\ 4 & 13.2 \pm 3.3 & 4.6 \pm 1.6 & 11.4 \pm 2.6 \\ 5 & 5.0 \pm 1.2 & 2.5 \pm 1.7 & 3.4 \pm 1.1 \\ 6 & 6.6 \pm 2.4 & 3.4 \pm 2.3 & 3.2 \pm 0.8 \\ 7 & 6.3 \pm 2.0 & 1.6 \pm 0.8 & 5.2 \pm 1.7 \\ 8 & 10.5 \pm 3.1 & 1.9 \pm 0.7 & 8.0 \pm 0.7 \\ & & & \\ \text { donors } & 8.5 \pm 2.6 & 3.0 \pm 1.0 & 6.3 \pm 2.9\end{array}$
A11 donors
$8.5 \pm 2.6$
$3.0 \pm 1.0$
$6.3 \pm 2.9$ 
TABLE 4:

T-LYMPHOCYTE SUBSETS IN EPIDIDYMAL BIOPSIES FROM PATIENTS WITH TESTICULAR OBSTRUCTION

\begin{tabular}{|c|c|c|c|c|}
\hline Patient & $\begin{array}{l}\text { Leu } 4^{+} \text {cells } \\
(\mathrm{A} 11-\mathrm{T} \text { cells) }\end{array}$ & $\begin{array}{l}\text { Leu } 2 \mathrm{a}^{+} \text {cells } \\
\text { ( } \mathrm{T} \text { suppressor } \\
\text { cytotoxic) }\end{array}$ & $\begin{array}{c}\text { Leu } 3 \mathrm{a}^{+} \text {cells } \\
\text { ( } \mathrm{T} \text { helper/ } \\
\text { inducer) }\end{array}$ & $\begin{array}{l}\text { Leu } 2 a / \\
\text { Leu } 3 a\end{array}$ \\
\hline
\end{tabular}

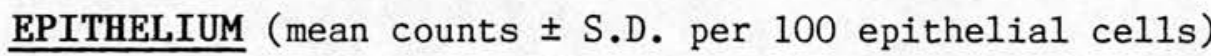

\begin{tabular}{crlll} 
HW & 7.39 & 7.22 & 1.51 & 4.78 \\
JK & 6.90 & 3.45 & 2.54 & 1.35 \\
BG & 10.87 & 8.60 & 2.59 & 3.32 \\
IG & 9.48 & 4.23 & 3.64 & 1.16 \\
DD & 7.35 & 3.56 & 2.52 & 1.41 \\
RT & 5.42 & 2.81 & 2.41 & 1.16 \\
A11 & $7.90 \pm$ & $4.97 \pm$ & $2.53 \pm$ & $2.19 \pm$ \\
patients & 1.95 & 2.35 & 0.67 & 1.51 \\
\hline
\end{tabular}

LAMINA PROPRIA (mean counts \pm S.D. per cross section of the epididymal duct)

\begin{tabular}{crccl} 
HW & $2.9 \pm 1.4$ & $1.6 \pm 0.7$ & $2.0 \pm 0.7$ & 0.60 \\
JK & $6.4 \pm 3.2$ & $2.1 \pm 1.7$ & $3.2 \pm 0.9$ & 0.74 \\
BG & $4.9 \pm 1.6$ & $2.0 \pm 0.8$ & $2.4 \pm 0.8$ & 0.08 \\
IG & $11.2 \pm 4.8$ & $0.8 \pm 0.6$ & $9.6 \pm 2.5$ & 0.83 \\
DD & $5.8 \pm 1.3$ & $3.5 \pm 1.6$ & $4.7 \pm 1.7$ & 0.80 \\
RT & $8.4 \pm 4.0$ & $3.6 \pm 1.6$ & $6.0 \pm 2.7$ & 0.65 \\
& & & & \\
A11 & $6.6 \pm$ & $2.26 \pm$ & $4.65 \pm$ & $0.61 \pm$ \\
donors & 2.88 & 1.09 & 2.84 & 0.27 \\
\hline
\end{tabular}

INTERSTITIAL TISSUES (mean counts \pm S.D. per $0.8 \mathrm{~mm}^{2}$ tissue $\times 160$ )

$\begin{array}{rlrll}\text { HW } & 20.0+5.7 & 5.7+1.6 & 15.4+5.6 & 0.37 \\ \text { JK } & 25.6+9.8 & 4.2+1.8 & 23.1+9.2 & 0.18 \\ \text { BG } & 48.5+12.0 & 14.5+4.0 & 38.0+11.8 & 0.38 \\ \text { IG } & 20.9+5.7 & 5.8+1.8 & 15.0+4.2 & 0.38 \\ \text { DD } & 42.0+20.0 & 10.0+2.1 & 34.8+11.4 & 0.28 \\ \text { RT } & 55.0+14.5 & 11.7+3.2 & 45.2+11.6 & 0.25 \\ & & & & \\ \text { A11 } & 35.3 \pm & 8.61 \pm & 28.6 \pm & 0.30 \pm \\ \text { patients } & 15.1 & 4.0 & 12.6 & 0.08\end{array}$


APPENDIX C 


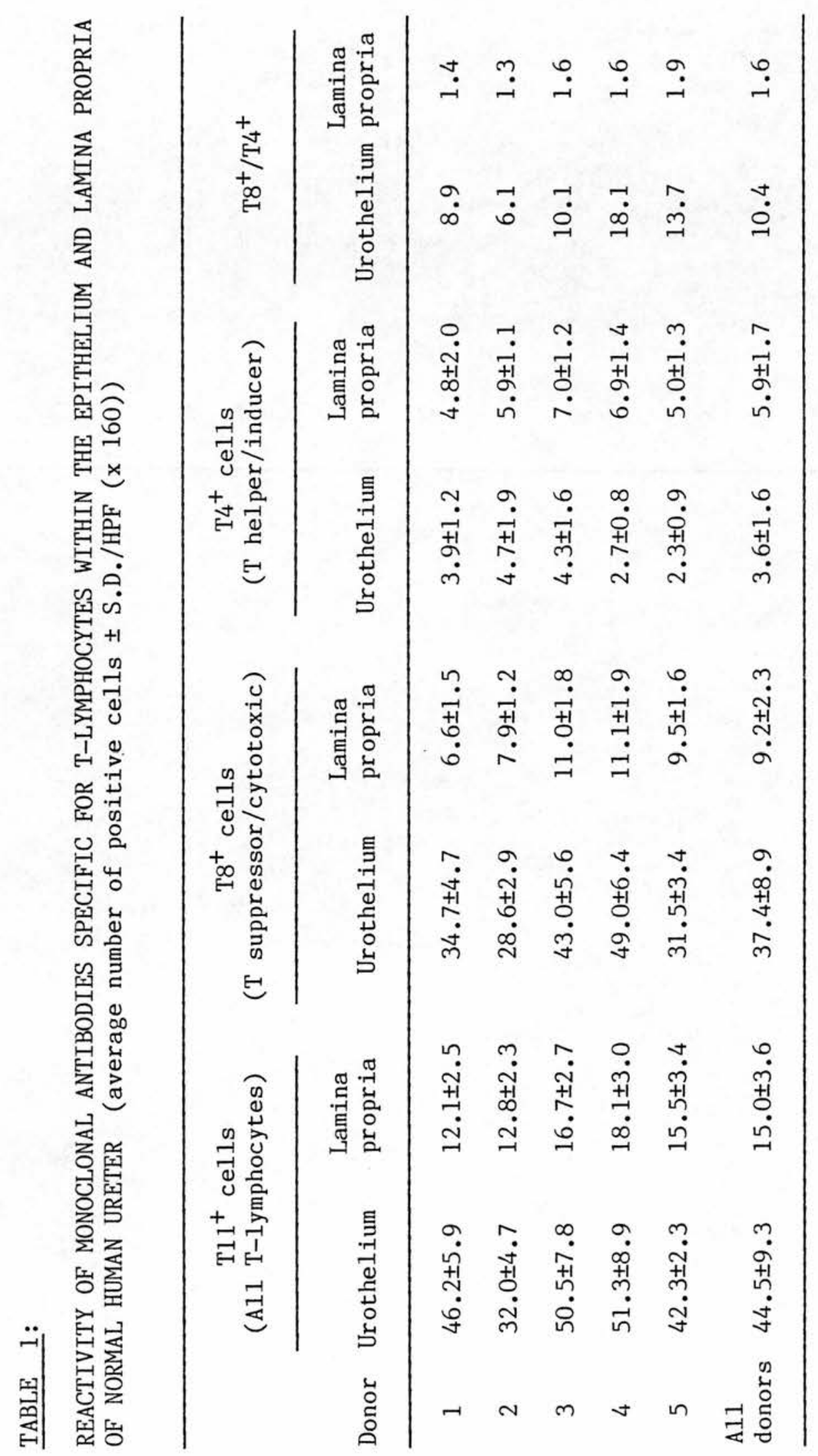




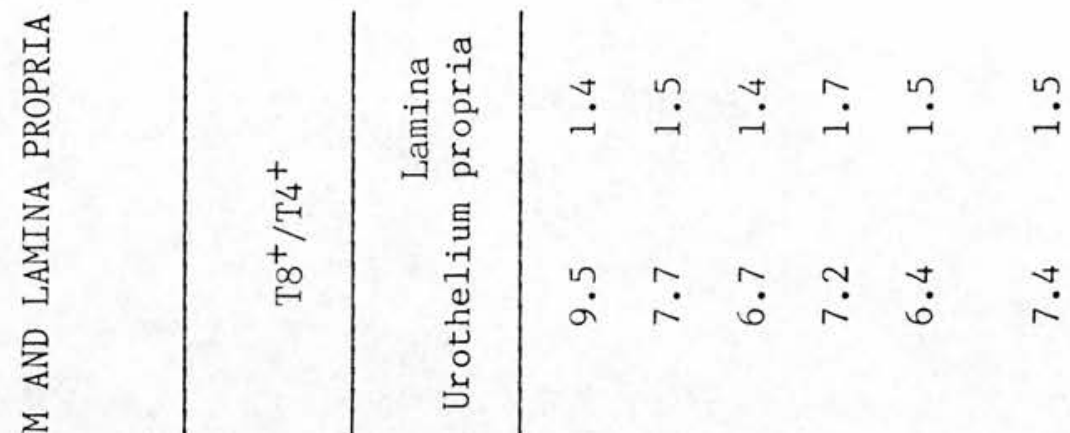

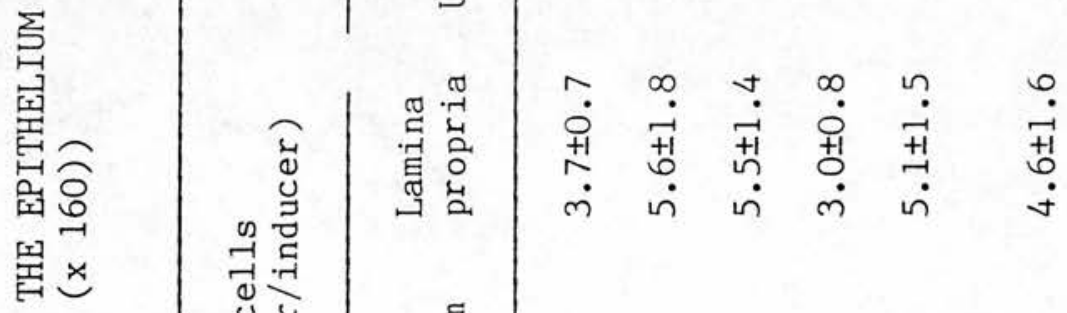

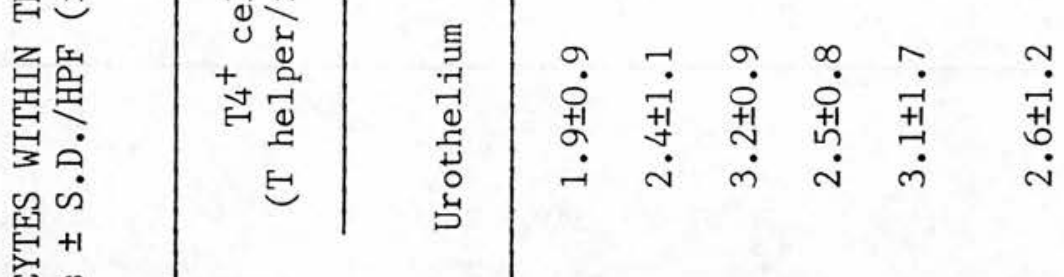

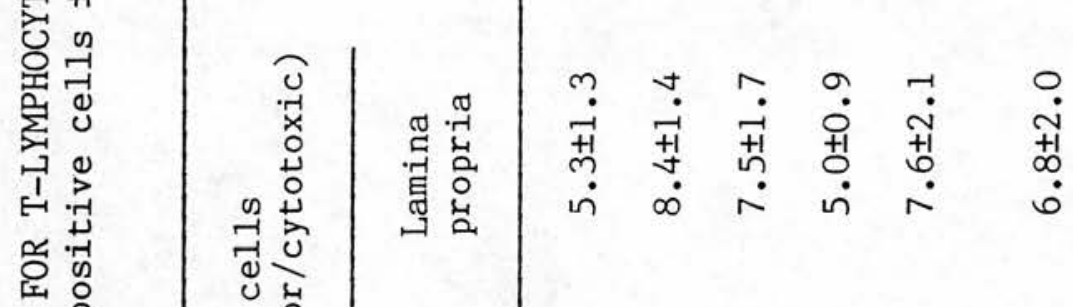

$$
\begin{aligned}
& 0 \\
& \text { 画㟔 } \\
& \text { 萬 } \\
& \text { 额 } \\
& \text { o }
\end{aligned}
$$

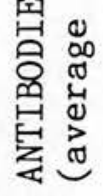

$$
\begin{aligned}
& \text { 要零 }
\end{aligned}
$$

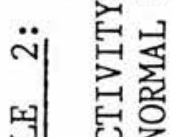

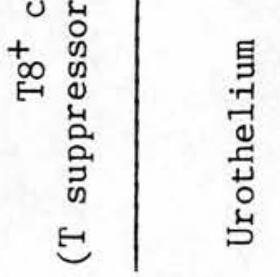

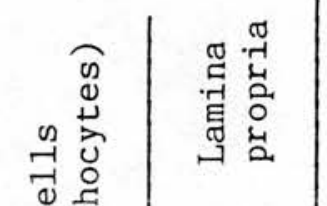

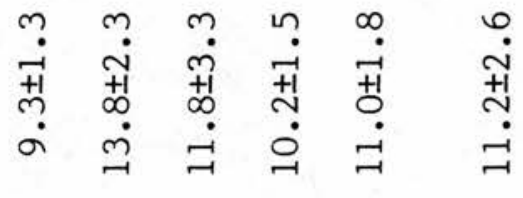

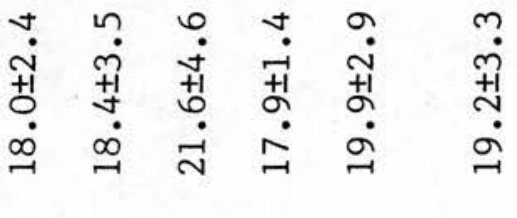

$$
\begin{aligned}
& + \\
& \text { 声要 }
\end{aligned}
$$

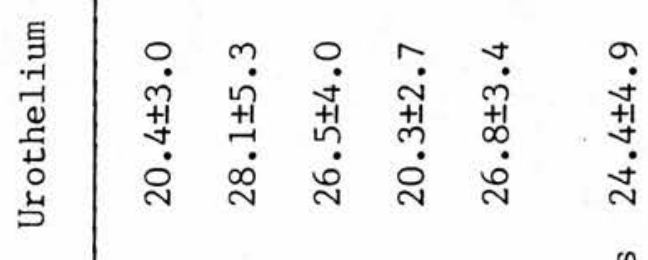

$$
\begin{aligned}
& \text { 范 }
\end{aligned}
$$


Details of published papers based on this study are as follows and reprints of journal articles are included.

\section{Journal Articles}

EL-DEMIRY, M.I.M., Hargreave, T.B., Busuttil, A., James, K., Ritchie, A.W.S. and Chisholm, G.D. (1985) Lymphocyte sub-populations in the male genital tract. British Journal of Urology, 57:769-774.

EL-DEMIRY, M.I.M., Hargreave, T.B., Busuttil, A., James, K. and Chisholm, G.D. (1986) Immunohistochemical identification of lymphocyte subsets and macrophages in normal human urothelium using monoclonal antibodies. British Journal of Urology, $58: 436-442$.

EL-DEMIRY, M.I.M., Young, H., Elton, R.A., Hargreave, T.B., James, K. and Chisholm, G.D. (1986) Leucocytes in the ejaculate from fertile and infertile men. British Journal of Urology, $58: 715-720$.

EL-DEMIRY, M.I.M., Hargreave, T.B., Busuttil, A., James, K. and Chisholm, G.D. (1986) Identifying leucocytes and leucocyte subpopulations in semen using monoclonal antibody probes. Urology, 23:492-496.

\section{Book Chapters}

Hargreave, T.B., James, K., Chisholm, G.D., EL-DEMIRY, M., Szymaniec, S., Harvey, J. and Ritchie, A.W.S. (1986)

Mechanisms of immunity within the prostate and male genital tract. In: W. Krause and C.F. Rothauge (eds.) Therapy of Prostatis. Klinische und Experimentee, Urologie II, pp. 123-126. Zuckschwerdt Verlag Muncheim.

Hargreave, T.B., Young, H., Wood, I.W., Harvey, J. EL-DEMIRY, M., James, K. and Chisholm, G.D. (1986) The role of Ureaplasma urealyticum in male infertility. In: W. Krause and C.F. Rothauge (eds.) Therapy of Prostatis. Klinische und Experimentee, Urologie II, pp. 230-235. Zuckschwerdt Verlag Muncheim.

\section{Accepted for publication}

EL-DEMIRY, M.I.M., Hargreave, T.B., Busuttil, A., James, K. and Chisholm, G.D. T-1ymphocyte subsets in human epididymis. Proceedings of the XII World Congress on Fertility and Sterility (Singapore, 1986).

EL-DEMIRY, M.I.M., Hargreave, T.B., Busuttil, A., Elton, R. James, K. and Chisholm, G.D. Immunocompetent cells in human testis in health and disease. Fertility and Sterility. 


\title{
Lymphocyte Sub-populations in the Male Genital Tract
}

\author{
M. I. M. EL-DEMIRY, T. B. HARGREAVE, A. BUSUTTIL, K. JAMES, A. W. S. RITCHIE and G. D. \\ CHISHOLM \\ University Department of Surgery/Urology and Department of Pathology, Western General Hospital, Edinburgh
}

\begin{abstract}
Summary - A series of monoclonal antibodies that react with human lymphocyte subsets was used in an indirect immunoperoxidase technique to study representative blocks from normal human testis, epididymis, vas deferens, prostate and seminal vesicles. Biopsies of testis, epididymis and vas obtained during surgical procedures directed at the investigation and treatment of infertile males were also studied. In all normal tissues, apart from the peripheral testis where no lymphocytes were identified, T lymphocytes were the predominant cell type (Leu $4+$ ). These lymphocytes were largely of the suppressor/cytotoxic phenotype (Leu $2 \mathrm{a}+$ ) and were more abundant in between the epithelial cells in the rete testis, epididymis, vas deferens, seminal vesicles and prostatic acini. Cells of the helper/inducer phenotype (Leu $3 a+$ ) were identified mainly within the interstitium of the epididymis and the prostate. B-lymphocytes (Leu 12+) were few in number and were mainly in the stroma of the prostate. In each organ the ratio of the T-cell subsets was determined and changes in this ratio were observed in epididymal and vasal biopsies from some infertile males. Finally, in testis biopsies from infertile men, suppressor/cytotoxic T-cells were demonstrated between the germinal epithelium and the fibrous tunica of the seminiferous tubules and as focal aggregates in the interstitium.
\end{abstract}

Immunogenicity of sperm cells and their ability to induce specific auto- and iso-immunity were reported as early as the turn of this century (Metlanikoff, 1900). Autoimmunity as a cause of infertility in men was initially reported by Rumke in 1954.

$\mathrm{B}$ and $\mathrm{T}$ lymphocytes are required for the generation of immune responses. B-lymphocytes are responsible for the expression of the humoral immunity, the production of antibodies, while T-lymphocytes are the main effector cells of cell mediated immune reactions. Two distinct subsets of human peripheral $\mathrm{T}$-cells have recently been characterised by monoclonal antibodies. They control both the type and intensity of cellular and humoral immune responses by acting as helper/ inducer or suppressor/cytotoxic cells (Reinherz and Schlossman, 1980). Therefore, determination of the

Read at the 41st Annual Meeting of the British Association of Urological Surgeons in Eastbourne, July 1985 precise location of lymphocyte subset populations within the tissues of the male genital tract is of considerable importance in understanding the autoimmune infertility. In the present study we have demonstrated and characterised the lymphocytes in testis, epididymis, vas deferens, prostate and seminal vesicles using monoclonal antibodies to human lymphocytes in an immunoperoxidase technique.

\section{Materials and Methods}

Tissue

Specimens of normal human testis, epididymis, vas deferens, prostate and seminal vesicles were obtained from five multi-organ transplant donors. Specimens of the vas deferens were also obtained from vasectomy patients. Surgical biopsies of testis (10) and epididymis (5) were obtained from patients under investigation and treatment of infertility.

Tissues were frozen in liquid nitrogen within 1 to 
$2 \mathrm{~h}$ of surgical removal and stored at $-70^{\circ} \mathrm{C}$ until used. Several blocks were prepared from each tissue and $5 \mu \mathrm{m}$ cryostat sections were cut from each block. Sections were air dried for 1 to $2 \mathrm{~h}$ under a hair dryer and kept at $-20^{\circ} \mathrm{C}$ until used.

\section{Antisera}

These included the monoclonal antibodies* antiLeu 4 which defines the entire population of T-lymphocytes, anti-Leu 3a which defines the helper/ inducer T-cell subset, anti-Leu 2a which defines the suppressor/cytotoxic T-cell subset and anti-Leu 12 which defines B-lymphocytes. The specificities of these antibodies have previously been described (Engleman et al., 1981; Ledbetter et al., 1981).

\section{Immunoperoxidase Technique}

The indirect labelled antibody method was applied (Nakane and Pierce, 1966). Immediately before staining the frozen sections were fixed in acetone for $10 \mathrm{~min}$, air dried and washed in $0.05 \mathrm{M}, \mathrm{pH} 7.6$ Tris- $\mathrm{HCl}$ buffer saline (TBS). Serial sections from each block were overlayed with $60 \mu \mathrm{l}$ of the appropriate dilutions of the monoclonal antibodies for $30 \mathrm{~min}$. Negative control sections were treated with TBS only. As positive controls the monoclonal antibodies were applied to frozen sections of lymph nodes. Subsequently all sections were incubated with peroxidase conjugated rabbit anti-mouse immunoglobulin (Dakopatt A/S) for $30 \mathrm{~min}$ diluted $1 / 50$ in TBS. All incubations were performed in moist chambers at room temperature. Between incubations, the sections were washed for $5 \mathrm{~min}$ in two changes of TBS. The final reaction was achieved by incubating the sections with $0.01 \%$ hydrogen peroxide and $0.05 \%$ diaminobenzidine tetrahydrochloride freshly prepared in TBS for 5 to $8 \mathrm{~min}$. The sections were then rinsed in tap water and counter stained in Mayer's haematoxylin for $30 \mathrm{~s}$, washed in tap water and mounted in Apathy's mounting medium.

\section{Immunohistological Analysis and Scoring}

The sections were examined by light microscopy using an X25 objective lens. The positively labelled cells were identified by their dark brown cell membrane staining and were scored within the epithelium, within the lamina propria and within the interstitial connective tissues. In almost every case the number of cells reacting with any individual

\footnotetext{
* These monoclonal antibodies were obtained from Becton Dickinson, Mechelen, Belgium.
}

monoclonal antibody was determined in 20 crosssections using an eye-piece with a graticule. The total number of the mucosal epithelial cells in certain sections was also determined by counting cell nuclei. On the basis of these counts we were able to determine (a) the percentage of the mucosal epithelial cells - in certain tissues - which stained with individual monoclonal antibodies and (b) the ratio of the helper/inducer T-cells to the suppressor/ cytotoxic T-cells in various compartments in each organ.

\section{Results}

\section{The Testis}

None of the antilymphocyte monoclonal antibodies used gave a detectable reaction with normal peripheral testis.

In marked contrast, T-lymphocytes (Leu 4+ cells) were always demonstrated in the rete testis with a predominance of cells of the suppressor/ cytotoxic phenotype (Leu $2 \mathrm{a}+$ ) (Table 1).

Table 1 Suppressor/Cytotoxic T-Lymphocytes (Leu $2 \mathrm{a}+)$ as a Percentage of the Total T-Cell Population (Leu 4+) within the Epithelium of the Male Genital Tract (Leu $2 \mathrm{a}+/$ Leu $4+$ )

\begin{tabular}{ll}
\hline Tissue & \% of T S/C \\
\hline Rete testis & $61.0(59.4-62.7)$ \\
Epididymis & $80.7(77.5-84.3)$ \\
$\quad$ Head & $79.1(72.4-84.1)$ \\
$\quad$ Body & $72.7(62.6-80.3)$ \\
Tail & $65.9(60.3-71.5)$ \\
Vas deferens & $68.4(52.1-84.7)$ \\
Prostate & $62.0(61.7-62.8)$ \\
\hline Seminal vesicles & \\
\hline
\end{tabular}

While no lymphocytes were detected in the normal peripheral testis, T-lymphocytes were demonstrated in 10/10 testicular biopsies obtained from infertile patients. These T-lymphocytes were almost always of the suppressor/cytotoxic phenotypes (Leu $2 \mathrm{a}+$ ). They were found in two locations. Isolated cells were noted between the fibrous tunica propria and the germinal epithelium of the seminiferous tubules. No cells were identified within the germinal epithelium. The other lymphocytes were arranged in small aggregates and follicles within the intertubular connective tissues (Fig. 1).

\section{The Epididymis}

In normal tissues no Leu $12+$ cells (B-cells) were 


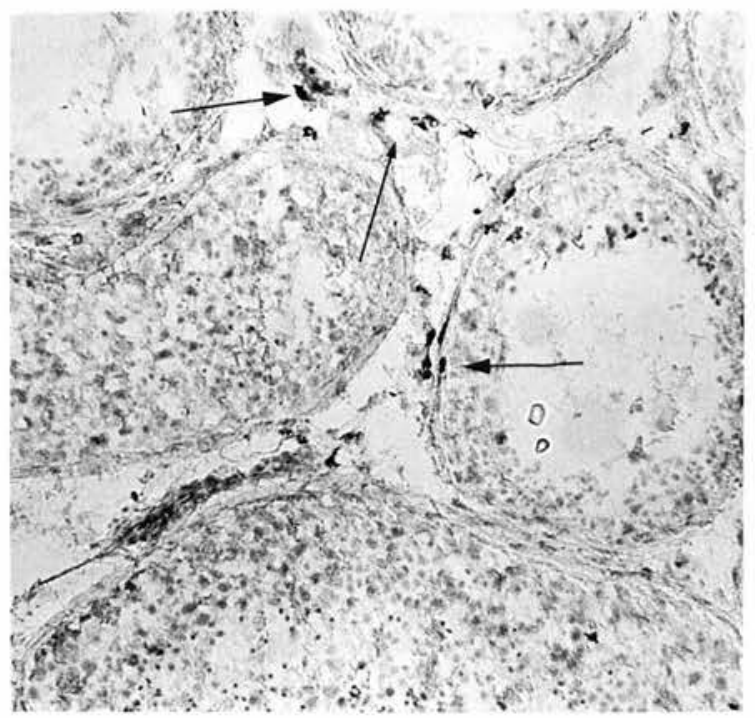

Fig. I Immunoperoxidase staining of a testicular biopsy from a sub-fertile patient with idiopathic oligozoospermia. Leu $2 \mathrm{a}+$ cells (suppressor/cytotoxic T-lymphocytes) are seen in the outer layer of the wall of the seminiferous tubules and in the interstitium. (Cryostat section, immunoperoxidase $\times 28$ ).

seen. Leu $4+$ cells (T-lymphocytes) were the predominant cell type. They were estimated to represent $12.7 \%$ of the mucosal cell population in the epithelium. Up to $77.9 \%$ of these T-lymphocytes were of the suppressor/cytotoxic phenotype (Leu $2 \mathrm{a}+$ ) (Table 1, Fig. 2a). T-lymphocytes of the helper/inducer phenotype $($ Leu $3 \mathrm{a}+$ ) were more abundant in the interstitium (Fig. 2b). Detailed results for the epididymis have been reported by ElDemiry et al. (1985).

\section{The Vas Deferens}

In the scrotal portion of the vas deferens, a Tlymphocyte population (Leu 4+) was demonstrated in between the epithelial cells and within the lamina propria. These T-lymphocytes constituted $25 \%$ of the epithelial cell population. Up to $66 \%$ of this T-cell population were suppressor/cytotoxic Tcells (Leu 2a+) (Fig. 3). However, the ratio of the suppressor/cytotoxic T-lymphocytes to the helper/ inducer T-lymphocytes was very close to that in the epididymis (Table 2 ). This ratio was changed in surgical biopsies obtained from patients during vasectomy reversal operations where marked increases of helper/inducer T-cells $($ Leu $3 \mathrm{a}+$ ) were noted.

\section{The Ampulla of the Vas and Seminal Vesicles}

In these sites, few T-lymphocytes were demon- strated and they were mainly of the suppressor/ cytotoxic phenotype (Leu $2 \mathrm{a}+$ ). They were located within the convoluted epithelial lining of both the ampulla of the vas and the seminal vesicles (Table 1).

\section{The Prostate}

No hyperplastic or inflammatory changes were seen in the specimens examined. The ducts and other acinar tissues were lined by columnar epithelium.

Lymphocytes were demonstrated within the epithelial lining, within the lumina of the ducts in proximity to the corpora amylacea and in immediately periductal connective tissues as well as in the intertubular connective tissue septa (Fig. 4a). The intra-epithelial lymphocytes were almost all Tlymphocytes and largely of suppressor/cytotoxic phenotype (Table 1). They tended to lie close to the basement membrane but very occasionally some cells appeared further up, in between the epithelial cells towards the lumen (Fig. 4b).

The interstitial positively labelled cells were more abundant and were mainly T-cells of the helper/ inducer phenotype. They tended to be arranged in clumps rather than singly. Similarly, there was a tendency to aggregation of lymphocytes within the interlobar septa of connective tissue; B-cell aggregations (Leu $12+$ ) were also seen in the interstitial tissues.

\section{Discussion}

Lymphocytes have been reported in the epithelium of the intestinal tract (Ferguson, 1977; Selby et al., 1981), the respiratory tract (Beinenstock et al., 1973) and the reproductive tract (Reid and Cleland, 1957; Hamilton, 1972; Hoffer et al., 1973; Dym and Romrell, 1975). It has been suggested that these lymphocytes are more likely to be present in every mucosal epithelial layer forming a common mucosal immunological system (Bienenstock et al., 1980). Ritchie et al. (1984) first used monoclonal antibodies to study lymphocyte distribution within the epididymis and the colour illustrations in that report clearly illustrate the striking results obtained by the immunoperoxidase technique.

In this study we have demonstrated and characterised lymphocytes along the length of the human male genital tract. Lymphocytes were almost always T-cells and they tended to be concentrated in large numbers in the epididymis, the vas deferens and the prostate respectively. They were few in 

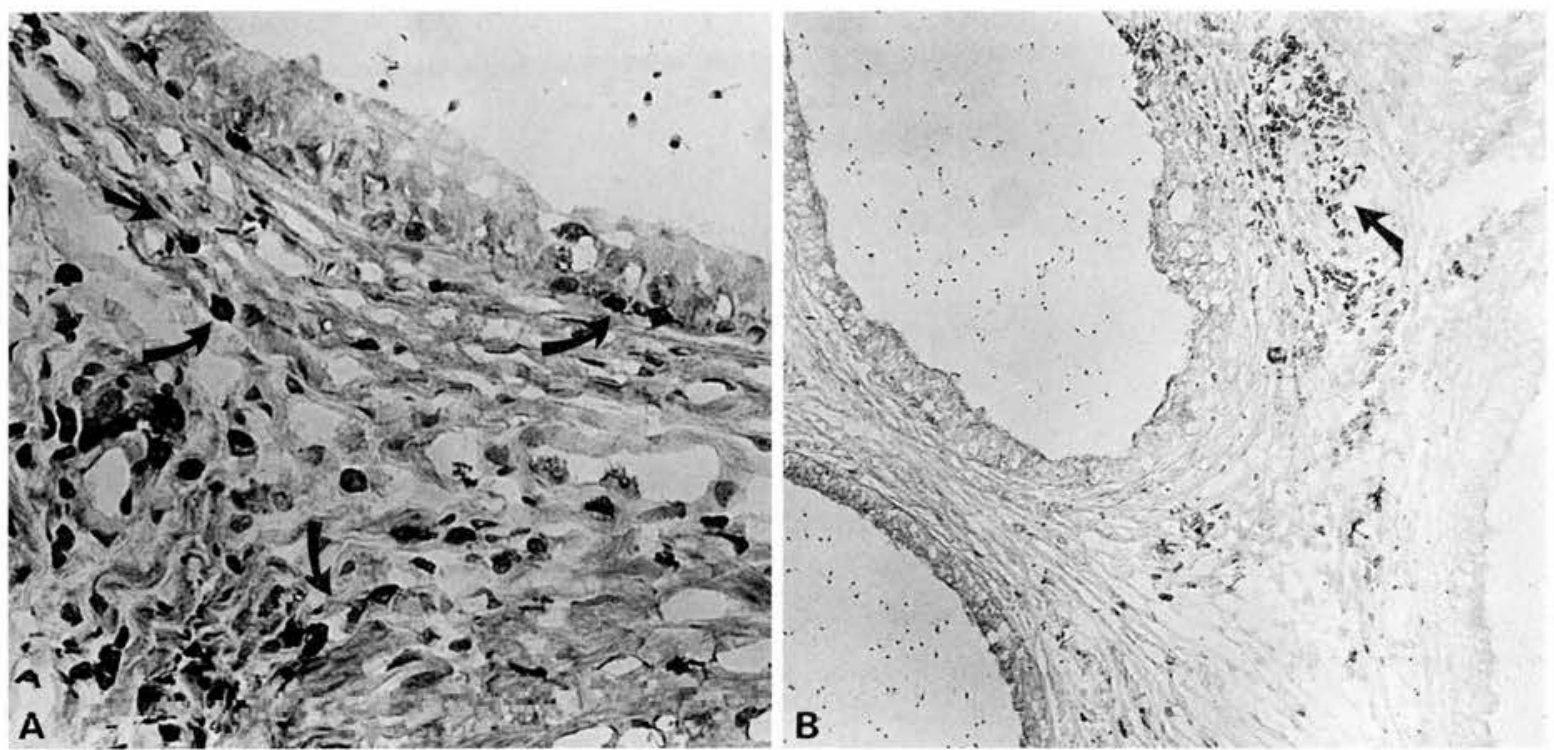

Fig. 2 (A) Human epididymis stained with anti-Leu 4 (Pan T-cells marker). T-lymphocytes are seen in the epithelium, lamina propria and in the interstitium. (Cryostat sections, immunoperoxidase $\times 64$ ). (B) Human epididymis stained with anti-Leu 3a (which defines helper/inducer T-lymphocytes). These $\mathrm{T} \mathrm{H} / \mathrm{Ind}$ lymphocytes are more abundant in the interstitium. (Cryostat sections, immunoperoxidase $\times 25$ ).

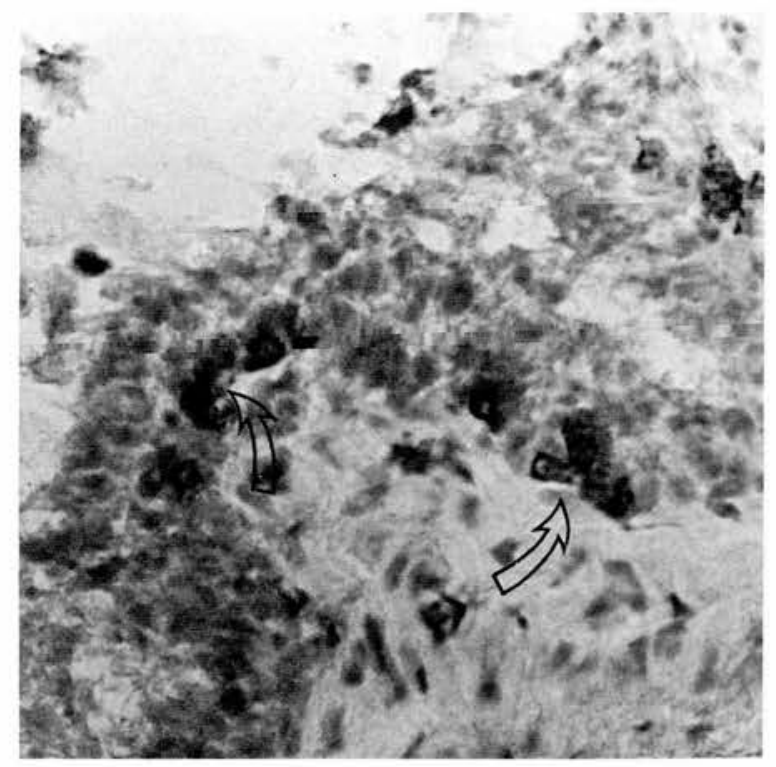

Fig. 3 Leu $2 \mathrm{a}+$ cells (suppressor/cytotoxic T-lymphocytes) in the lining epithelium of the human vas deferens. (Cryostat section, immunoperoxidase $\times 77$ ).

number in the rete testis, ampulla of the vas and seminal vesicles. B-lymphocytes were demonstrated only in the stroma of the prostate.

T-lymphocytes exhibited a compartmentalised
Table 2 Average Ratio of Suppressor/Cytotoxic TLymphocytes to Helper/Inducer T-Lymphocytes in Normal Male Genital Tract

\begin{tabular}{lccc}
\hline Tissuc & $\begin{array}{l}\text { Intra- } \\
\text { epithehal }\end{array}$ & $\begin{array}{l}\text { Lamina } \\
\text { propria }\end{array}$ & $\begin{array}{l}\text { Interstitial } \\
\text { tissuc }\end{array}$ \\
\hline Rete testis & 2.1 & - & 0.6 \\
Epididymis & $(1.8-2.3)$ & & $(0.5-0.7)$ \\
Head & 4.1 & 1.4 & 0.5 \\
Body & $(3.5-5.5)$ & $(1.1-2.3)$ & $(0.39-0.69)$ \\
Tail & 3.0 & 1.3 & 0.6 \\
Vas deferens & $(2.5-3.5)$ & $(1.0-2.3)$ & $(0.35-0.74)$ \\
& 2.4 & 1.7 & 0.4 \\
Prostate & $(2.2-2.8)$ & $(1.6-2.3)$ & $(0.37-0.51)$ \\
Seminal & $(1.2-2.4)$ & $(1.2-1.9)$ & \\
vesicles & 2.6 & - & 3.9 \\
\hline
\end{tabular}

(Ratio in peripheral blood is 0.5 )

distribution. Within the epithelium, lymphocytes of the suppressor/cytotoxic phenotype were the major component. This was observed at all levels of the genital tract (Table 1). Suppressor/cytotoxic T-cells were also preponderant in the lamina propria of the epididymis, vas deferens and seminal vesicles. In contrast, the helper/inducer T-lympho- 

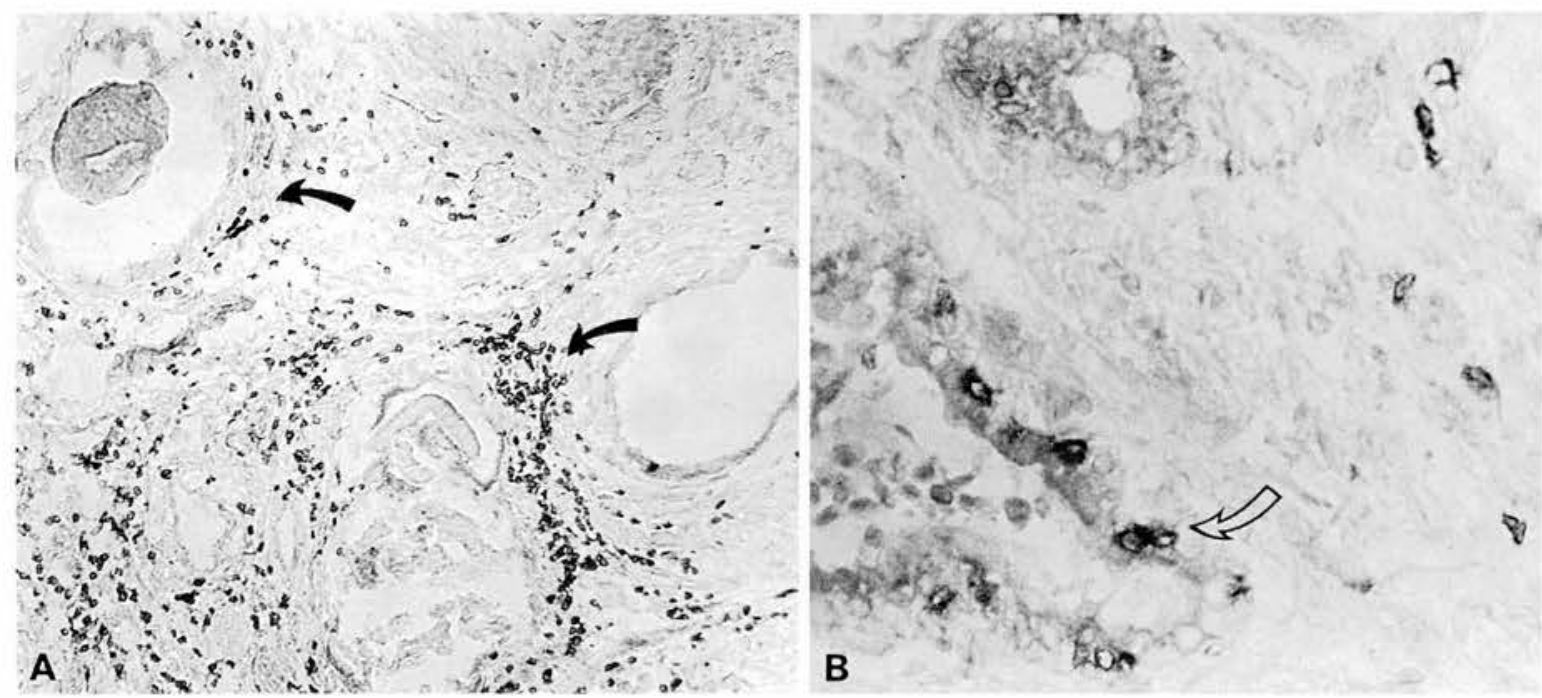

Fig. 4 (A) Leu $4+$ cells (T-lymphocytes) in normal human prostate are seen in the lining epithelium of the prostatic acini, in lumina ducts and in the interstitium. (Immunoperoxidase $\times 40$ ). (B) Leu $2 \mathrm{a}+$ cells (suppressor/cytotoxic T-lymphocytes in the lining epithelium of prostatic acini. (Immunoperoxidase $\times 65$ ).

cytes were more abundant in the interstitial connective tissues (Table 2). It is most likely that the predominant suppressor/cytotoxic T-cells in the epithelial lining of male genital tract function form a major immunological barrier which normally prevents the development of autohumoral or cellular immune responses to sperm antigens.

The pattern of distribution of this suppressor barrier is consistent with the functional properties of each organ, namely the absence of the lymphocytes from the normal peripheral testis, where there is an anatomical blood-testis barrier formed by the Sertoli cells and which appears only at puberty (Kormano, 1967; Vitale et al., 1973; Setchell, 1980). Freund and Davis (1969) reported that 60 to $70 \%$ of the spermatozoa within a normal ejaculate come from the epididymis and proximal part of the vas deferens. The testicular fluid is also absorbed in the epididymis. Since it is well established that human sperm are immunogenic, the absorption of the products of sperm breakdown in the epididymis represents an enormous immunogenic stimulation which will lead to initiation of immune responses. But it seems that under the conditions of a healthy genital tract, this sperm-immunogenic stimulation can be easily handled by the immunological suppressor barrier $\left(\mathrm{T}_{\mathrm{sic}}\right)$ in the lining epithelium of the epididymis and to a certain extent in the vas deferens. This may also explain why the suppressor/cytotoxic T-cells are more abundant in the epididymis than the rest of the genital tract. The occurrence of sperm granuloma, testicular degeneration and the development of autoimmunity post-vasectomy and post-obstruction may illustrate the importance of the vas deferens and its epithelial suppressor barrier in the normal immune homeostasis of the male genital tract.

A potential consequence of this immunological suppressor barrier in the prostate is that infections and inflammations are commonly encountered in young men. Inflammation may result in occlusion of the ejaculatory duct and absorption of prostatic and sperm antigens. The demonstration of B-lymphocytes in the interstitial connective tissues of the prostate may account for the source and level of local secretion of immunoglobulins.

The finding that the epithelium of seminal vesicles contains few suppressor T-cells is also consistent with its function. The seminal vesicles are not a storage depot for spermatozoa and the secretions of the seminal vesicles are only in contact with the spermatozoa at the moment of ejaculation (Clavert et al., 1985), i.e. there is little or no absorption of sperm antigen.

In conclusion, lymphocyte subpopulations were normally demonstrated in rete testis, epididymis, vas deferens, prostate and seminal vesicles, with a specific pattern of distribution in the epithelium, lamina propria and the interstitium. T-lymphocyte of suppressor/cytotoxic phenotype were found to constitute the larger part of the T-cell population along the epithelial lining of the genital tract organs (Table 2). The ratio of $T_{s i c}$ to $T_{H}$ Ind was consistent in all of the specimens examined from 
each organ. Changes in this ratio were noted in clinical specimens but the true significance of this finding must await study of a larger number of abnormal specimens from patients with defined clinical problems.

\section{References}

Bienenstock, J., Befus, D. and McDermott, M. (1980). Mucosal immunity. Monographes in Allergy, 16, 1-18.

Bienenstock, J., Johnston, N. and Percy, D. Y. E. (1973). Bronchial lymphoid tissue. 1. Morphologic characteristics. Laboratory Investigations, 28, 686-692.

Clavert, A., Gabriel-Robez, O. and Montagnon, D. (1985). Physiological role of the seminal vesicle in seminal vesicles and fertility. Progress in Reproductive Biology and Medicine, 12, 80-94

Dym, M. and Romrell, L. J. (1975). Intraepithelial lymphocytes in the male reproductive tract of rats and rhesus monkeys. Journal of Reproduction and Fertility, 42, 1-7.

El-Demiry, M. I. M., Hargreave, T. B., Smith, G., Busuttil, A., James, K. and Chisholm, G. D. (1985). T-lymphocytes subsets in the human epididymis. In Proceedings of the Third International Forum of Andrology, Paris. (In press.)

Engleman, E. G., Benike, C. J., Glickman, E. and Evans, R. L. (1981). Antibodies to membrane structures that distinguish suppressor/cytotoxic and helper T-lymphocyte subpopulations block the mixed leucocyte reaction in man. Journal of Experimental Medicine, 154, 193-198.

Ferguson, A. (1977). Intraepithelial lymphocytes of the small intestine. Gut, 18, 921-937.

Freund, M. J. and Davis, J. E. (1969). Disappearance rate of spermatozoa from the ejaculate following vasectomy. Fertility and Sterility, 20, 163-170.

Hamilton, D. W. (1972). The mammalian epididymis. In Reproductive Biology, ed. Balin, H. and Glasser, S. Pp. 268-337. Amsterdam: Excerpta Medica.

Hoffer, A. P., Hamilton, D. W. and Fawcett, D. W. (1973). The ultrastructure of the principal cells and intraepithelial leucocytes in the initial segment of the rat epididymis. Anatomical Record, 175, 169-202.

Kormano, M. (1967). Dye permeability and alkaline phosphatase activity of testicular capillaries in the post-natal rat. Histochemistry, 9, 327-338.

Ledbetter, J. A., Evans, R. L., Lipinski, M., CunninghamRundles, C., Good, R. A. and Herzenberg, L. A. (1981). Evolutionary conservation of surface molecules that distinguish T-lymphocyte helper/inducer and T-cytotoxic/suppressor subpopulations in mouse and man. Journal of Experimental Medicine, 153, 310-323.
Metlanikoff, S. (1900). Etudes sur la spermotoxine. Annales de IInstitut Pasteur, 14, 577-589.

Nakane, P. K. and Pierce, G. B. (1966). Enzyme labelled antibodies: preparation and application for localisation of antigens. Journal of Histochemistry and Cytochemistry, 14, 929931.

Reid, B. L. and Cleland, K. W. (1957). The structure and function of the epididymis. I. The histology of the rat epididymis. Australian Journal of Zoology, 5, 223-246.

Reinherz, E. L. and Schlossman, S. F. (1980). The differentiation and function of human T-lymphocytes. Cell, 19, 821-827.

Ritchie, A. W. S., Hargreave, T. B., James, K. and Chisholm, G. D. (1984). Intra-epithelial lymphocytes in the normal epididymis. A mechanism for tolerance to sperm auto-antigens? British Journal of Urology, 56, 79-83.

Rumke, Ph. (1954). The presence of sperm antibodies with the serum of two patients with oligozoospermia. Vox Sanguinis, 4, 135-140.

Selby, W. S., Janossy, G., Goldstein, G. and Jewell, J. P. (1981). T-lymphocyte subsets in human intestinal mucosa: the distribution and relationship to MHC-derived antigens. Clinical and Experimental Immunology, 44, 453-458.

Setchell, B. P. (1980). The functional significance of the blood testis barrier. Journal of Andrology, 1, 3-10.

Vitale, R., Fawcett, D. W. and Dym, M. (1973). The normal developmental blood testis barrier and the effects of clomiphene and oestrogen treatment. Anatomical Record, 176, 333334.

\section{The Authors}

M. I. M. El-Demiry, MB,BCh(Cairo), MSc(Urol Cairo), Senior Resident in Urology, Police Hospital, Cairo, Egypt. Honorary Registrar, University Department of Surgery/Urology.

T. B. Hargreave, MS, FRCS, FRCSE, Senior Lecturer and Consultant Urologist, University Department of Surgery/ Urology,

A. Busuttil, MD, MRCPath, Consultant Pathologist, Western General Hospital.

K. James, DSc, FRCPath, FRSE, Reader in Surgery, University of Edinburgh.

A. W. S. Ritchie, MD, FRCSE, Senior Registrar, University Department of Surgery/Urology.

G. D. Chisholm, ChM, FRCS, FRCSE, Professor of Surgery and Consultant Urologist, Western General Hospital.

Requests for reprints to: M. I. M. El-Demiry, University Department of Surgery/Urology, Western General Hospital, Edinburgh EH4 2 XU. 


\title{
Immunohistochemical Identification of Lymphocyte Subsets and Macrophages in Normal Human Urothelium Using Monoclonal Antibodies
}

\author{
M. I. M. EI-DEMIRY, T. B. HARGREAVE, A. BUSUTTIL, K. JAMES and G. D. CHISHOLM \\ University Department of Surgery/Urology and Department of Pathology, Western General Hospital, Edinburgh
}

Summary-Monoclonal antibodies were used to examine the pattern of distribution of lymphocyte subpopulations and macrophages in normal human urothelium. T lymphocytes (T11-positive cells) were demonstrated within the epithelium and within the lamina propria of both the ureter and the urinary bladder. Suppressor/cytotoxic T cells (T8-positive cells) predominated, with the average ratio of suppressor/cytotoxic T cells to the helper/inducer T cells being approximately 8.9 in the epithelium and 1.5 in the lamina propria. Leu M3 + cells (monocytes/macrophages) were detected in both epithelium and lamina propria. The character and distribution of these immunocompetent cells within the urothelium suggest a protective function, especially against infection and in tumour surveillance.

The mucosal lining of the urinary tract is constantly being challenged by a variety of biological and chemical agents which include micro-organisms and toxic or carcinogenic substances. The development of mucosal as well as systemic immune responses to microbial and other antigens is controlled and regulated by specific immunoregulatory mechanisms. T lymphocytes and macrophages are known to play a major role in such immune regulation. Two distinct subsets of T lymphocytes, of the helper/inducer (T4-positive) and the suppressor/cytotoxic (T8-positive) phenotypes, have been defined in man using monoclonal antibodies (Reinherz and Schlossman, 1980). These subsets of $T$ cells modulate the activity of a variety of effector cells, including the natural killer cells (NK cells), cytotoxic T cells and B lymphocytes, that give rise to antibody-producing plasma cells (Reinherz et al., 1980). It is also widely accepted that the initiation of certain immune responses involves accessory cells such as macrophages/dendritic cells which are responsible for antigen presentation to lymphocytes (Friedman, 1981). T cells

Accepted for publication 10th March 1986 will recognise foreign antigens only when they are associated on the surface of a cell with a molecule encoded by the major histocompatibility complex (MHC). Thus cytotoxic cells usually see antigen in association with Class $1 \mathrm{MHC}$ (molecules which are expressed on all cells), and helper cells usually see antigen in the context of MHC Class II molecules (Ia or Dr-like) which are expressed on a number of the cell varieties which comprise the immune system (Robertson, 1985).

The recent development of specific monoclonal antibodies as well as highly sensitive immunohistochemical staining techniques has made it possible to study immunocompetent cells in tissue sections. We have therefore examined the distribution of lymphocyte subpopulations and macrophages in the tissues of normal ureter and urinary bladder using monoclonal antibodies in an indirect immunoperoxidase technique.

\section{Material and Methods}

\section{Tissues}

Specimens of normal ureter were obtained from five donors for living related kidney transplants 
Table 1 Monoclonal Antibodies Reacting with Human Lymphoreticular Cells

\begin{tabular}{lcl}
\hline Monoclonal antibody & Source & Specificity \\
\hline T11 & *Coulter Electronics & All T lymphocytes \\
T8 & Coulter Electronics & T suppressor/cytotoxic cells \\
T4 & Coulter Electronics & T helper/inducer cells \\
B1 & Coulter Electronics & B lymphocytes \\
Anti-Leu M3 & †ecton Dickenson & Monocytes/macrophages \\
Anti-1L2-receptors & Becton Dickenson & Interleukin 2-receptors on activated T cells \\
Anti-HLA-DR & Becton Dickenson & Class II molecule (Dr-Ia-like antigen) \\
\hline
\end{tabular}

* Coulter Electronics Ltd, Luton.

† Laboratory Impex Ltd, Twickenham.

and specimens of urinary bladder wall from the vault of the bladder from five brain-dead ventilated cadaver kidney donors who were victims of road traffic accidents. Permission for multi-organ donation had been granted by their next of kin. All tissues were snap frozen in liquid nitrogen within 1 to $2 \mathrm{~h}$ of surgical removal and stored at $-70^{\circ} \mathrm{C}$ until used. Serial cryostat sections ( $5 \mu \mathrm{m}$ thick) were cut, air dried for $1 \mathrm{~h}$, and fixed in acetone for $10 \mathrm{~min}$ at room temperature. The slides were wrapped in aluminium foil and kept at $-20^{\circ} \mathrm{C}$ until used.

\section{Monoclonal Antibodies}

Full details of the murine monoclonal antibodies used in this study are given in Table 1. The optimal working dilutions of these antibodies and the specificity and reliability of the staining technique were determined by staining similarly frozen sections of normal human tonsil, spleen and lymph nodes. These tissues were also used as positive controls in every staining session.

\section{Immunoperoxidase Staining}

The indirect immunoperoxidase technique was used throughout. Details of the procedure have been described by El-Demiry et al. (1985). Briefly, serial sections were incubated with the different primary mouse monoclonal antibodies, followed by peroxidase conjugated rabbit-anti-mouse antibody (Dakopatt, Copenhagen). In the case of negative controls the primary antibodies were omitted. The colour reaction was developed by incubating the sections with diaminobenzidine/hydrogen peroxide substrate. Sections were finally counterstained with haematoxylin, dehydrated and mounted in DPX.

\section{Immunohistological Analysis}

Sections were examined by light microscopy using

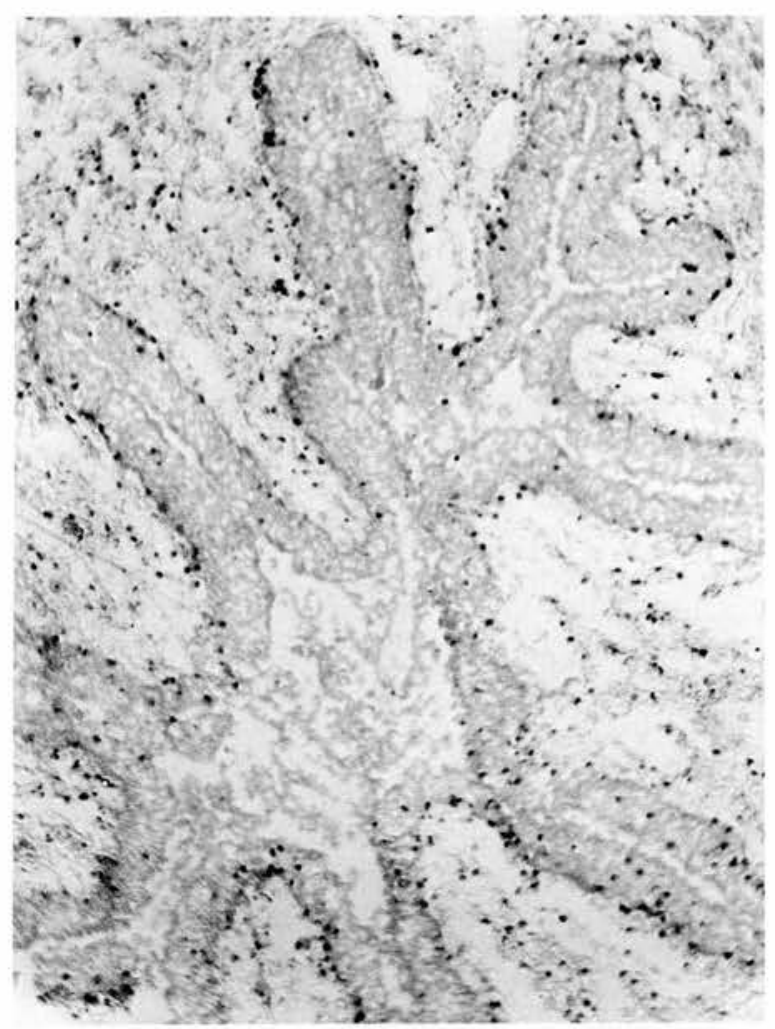

Fig. 1 Mucosal (epithelial and lamina propria) T lymphocytes (T11-positive cells) in normal human ureter. (Cryostat section: human peroxidase $\times 40$ ).

a $\times 160$ magnification. The positively labelled cells were identified by their dark brown cell membrane related staining and were counted both within the epithelium (urothelium) and within the subepithelial connective tissues (lamina propria) of both the ureter and the urinary bladder. In every case the number of cells reacting with any individual monoclonal antibody was determined in 10 different fields in identical areas of the serial sections. The results are expressed as the mean $( \pm S D)$ of the 


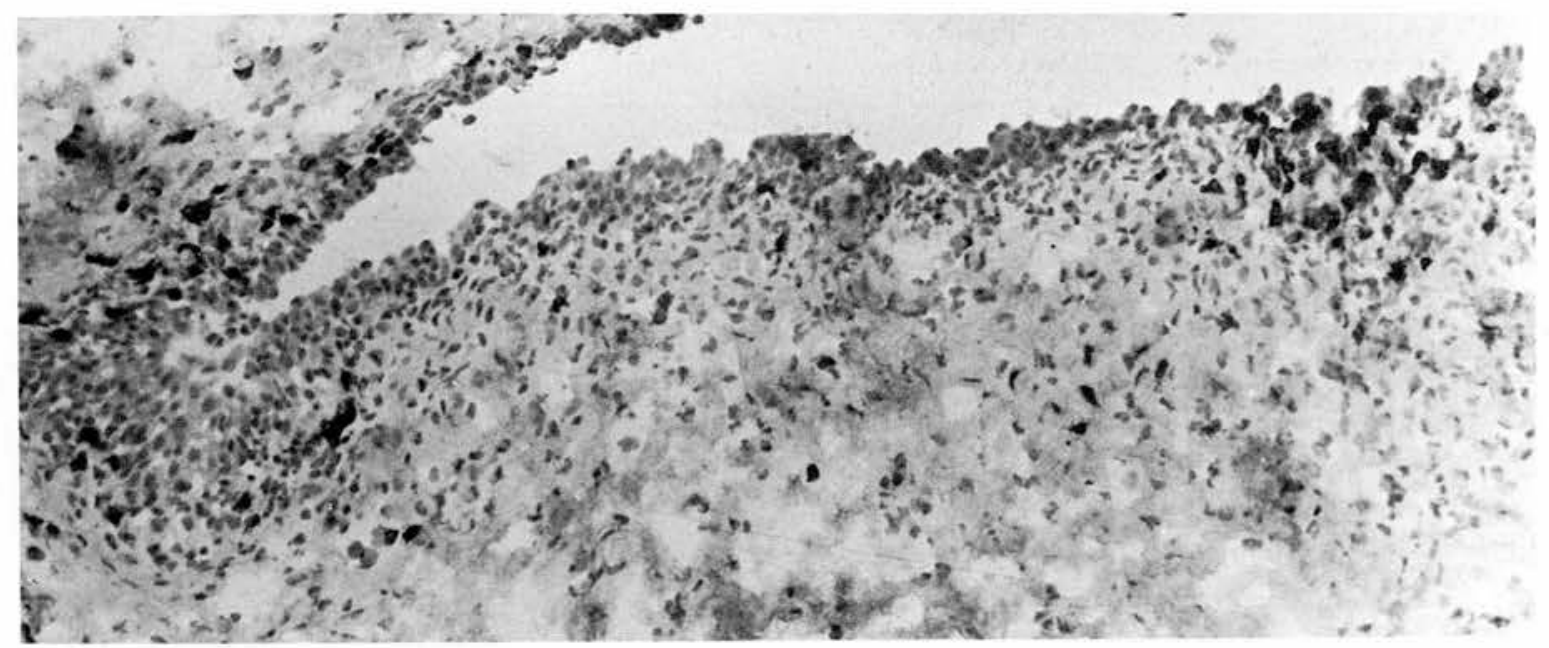

Fig. 2 T11-positive cells (T lymphocytes) in normal bladder mucosa. (Cryostat section: immunoperoxidase $\times 80$ ).

Table 2 Reactivity of Monoclonal Antibodies Specific for T Lymphocytes within the Epithelium and Lamina Propria of Normal Human Ureter (Average Number of Positive Cells $\pm \mathrm{SD} / \mathrm{HPF}(\times 160)$ )

\begin{tabular}{|c|c|c|c|c|c|c|c|c|}
\hline \multirow[b]{2}{*}{ Donors } & \multicolumn{2}{|c|}{$\begin{array}{l}\text { All T lymphocytes } \\
\text { (T11-positive) }\end{array}$} & \multicolumn{2}{|c|}{$\begin{array}{l}\text { Tsuppressor/cytotoxic cells } \\
\text { (T8-positive) }\end{array}$} & \multicolumn{2}{|c|}{$\begin{array}{l}\text { Thelper/inducer cells } \\
\text { (T4-positive) }\end{array}$} & \multicolumn{2}{|c|}{$\begin{array}{l}\text { Tsuppressor/cytotoxic } \\
\text { cells to Thelper/inducer } \\
\text { cells ratios } \\
\text { (T8-positive/T4-positive) }\end{array}$} \\
\hline & Urothelium & $\begin{array}{l}\text { Lamina } \\
\text { propria }\end{array}$ & Urothelium & $\begin{array}{l}\text { Lamina } \\
\text { propria }\end{array}$ & Urothelium & $\begin{array}{l}\text { Lamina } \\
\text { propria }\end{array}$ & Urothelium & $\begin{array}{l}\text { Lamina } \\
\text { propria }\end{array}$ \\
\hline 1 & $46.2 \pm 5.9$ & $12.1 \pm 2.5$ & $34.7 \pm 4.7$ & $6.6 \pm 1.5$ & $3.9 \pm 1.2$ & $4.8 \pm 2.0$ & 8.9 & 1.4 \\
\hline II & $32.0 \pm 4.7$ & $12.8 \pm 2.3$ & $28.6 \pm 2.9$ & $7.9 \pm 1.2$ & $4.7 \pm 1.9$ & $5.9 \pm 1 . i$ & 6.1 & 1.3 \\
\hline III & $50.5 \pm 7.8$ & $16.7 \pm 2.7$ & $43.0 \pm 5.6$ & $11.0 \pm 1.8$ & $4.3 \pm 1.6$ & $7.0 \pm 1.2$ & 10.1 & 1.6 \\
\hline IV & $51.3 \pm 8.9$ & $18.1 \pm 3.0$ & $49.0 \pm 6.4$ & $11.1 \pm 1.9$ & $2.7 \pm 0.8$ & $6.9 \pm 1.4$ & 18.1 & 1.6 \\
\hline V & $42.3 \pm 2.3$ & $15.5 \pm 3.4$ & $31.5 \pm 3.4$ & $9.5 \pm 1.6$ & $2.3 \pm 0.9$ & $5.0 \pm 1.3$ & 13.7 & 1.9 \\
\hline All Donors & $44.5 \pm 9.3$ & $15.0 \pm 3.6$ & $37.4 \pm 8.9$ & $9.2 \pm 2.3$ & $3.6 \pm 1.6$ & $5.9 \pm 1.7$ & 10.4 & 1.6 \\
\hline
\end{tabular}

number of positive cells in a single field (i.e. $\left.0.8 \mathrm{~mm}^{2}\right)$.

\section{Results}

The reactivity of the primary monoclonal antibodies with sections of human tonsils, spleen and lymph nodes (the positive control sections) was confirmed as similar to that previously reported (Poppema et al., 1981; Ritchie et al., 1983).

\section{T Lymphocytes}

$\mathrm{T}$ lymphocytes stained with the pan $\mathrm{T}$ cell monoclonal antibody (T11) were demonstrated within the mucosal lining of both the ureter and the urinary bladder. They showed a uniform distribution in the urothelium around the entire circumference of the ureter (Fig. 1), while cellular distribution within the bladder mucosa was patchy (Fig. 2). In both the ureter and the urinary bladder, the $\mathrm{T}$ lymphocytes (T11-positive) were situated mainly within the epithelium and the lamina propria, with the greater proportion being present within the epithelium (Tables 2 and 3 ). The T lymphocytes were situated alongside the epithelial cells at various levels above the basement membrane but tended to be concentrated towards the basal layer of the epithelium. In the lamina propria the T lymphocytes (T11-positive) cells were found towards the upper part of the sub-epithelial connective tissue layers, also in close proximity to the other surface of the basement membrane.

Most of these mucosal T lymphocytes were identified as T8-positive, i.e. of the suppressor/ cytotoxic phenotype (Figs 3 and 4). In the ureter, 
Table 3 Reactivity of Monoclonal Antibodies Specific for T Lymphocytes within the Epithelium and Lamina Propria of Normal Human Bladder (Average Number of Positive Cells $+\mathrm{SD} / \mathrm{HPF}(\times 160)$ )

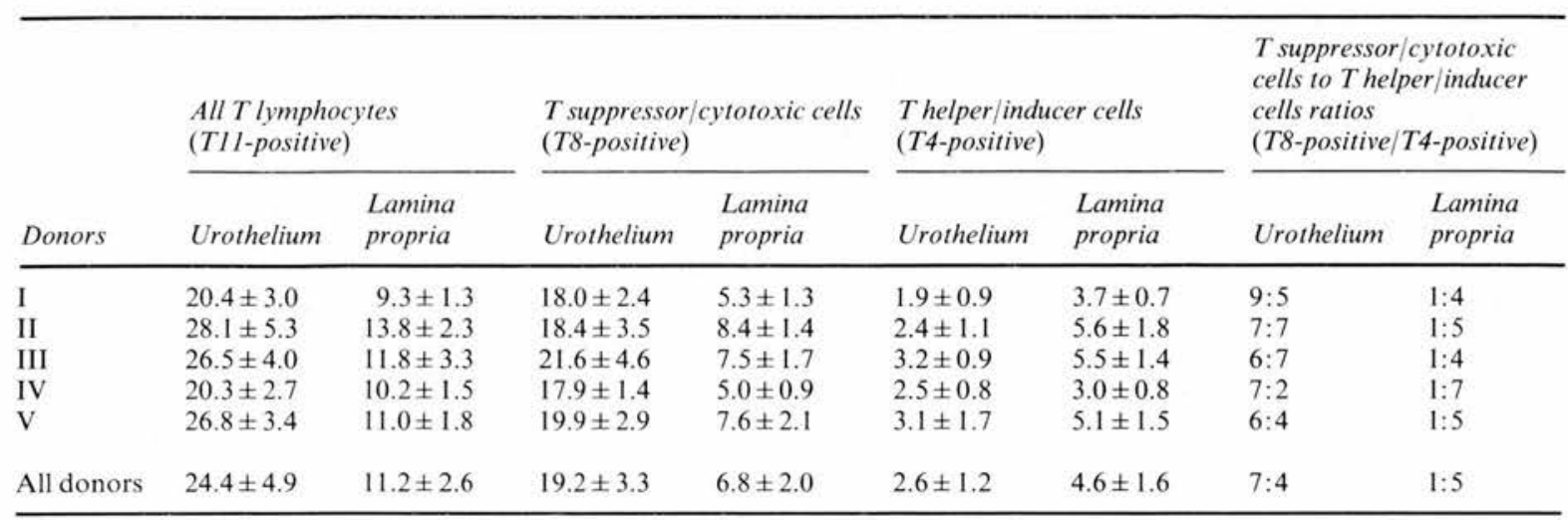

$84.1 \%$ of the intra-epithelial $\mathrm{T}$ lymphocytes and $61.3 \%$ of the lamina propria T lymphocytes exhibited the suppressor/cytotoxic phenotype. In the urinary bladder the distribution of $\mathrm{T}$ lymphocytes was similar; $87.7 \%$ of the intra-epithelial $\mathrm{T}$ cells and $60.7 \%$ of the lamina propria T cells expressed the suppressor/cytotoxic phenotype (Tables 2 and 3).

Some T4-positive cells (helper/inducer T lymphocytes) were also detected both within the epithelium and the lamina propria of the mucosal lining of the ureter and the urinary bladder and were located mainly in the lamina propria. They were fewer in number than the T8-positive cells. The average ratios of the suppressor/cytotoxic $T$ cells to the helper/inducer T cells (T8-positive/T4positive) were $10: 4$ and $7: 4$ in the urothelium of the ureter and the urinary bladder respectively. None of the T lymphocytes showed a detectable reaction with the anti-interleukin-2 receptor monoclonal antibody which detects activated T cells.

\section{B Lymphocytes}

Cells reacting with the $\mathrm{B} 1$ antibody which detects $\mathrm{B}$ lymphocytes were seen as small lymphoid aggregates in the outer part of the serosa of the ureter. No B1-positive cells were detected in the mucosal lining of the ureter and the urinary bladder.

\section{Monocytes and Macrophages}

Cells stained by the anti-Leu M3 monoclonal antibody, which reacts with cell-surface antigens of monocytes/macrophages, were few in number and were detected mainly in the lamina propria. Occasional Leu M3 + cells were also seen within the urothelium.

\section{Anti HLA-DR reactive cells}

Most of the mucosal cells were HLA-DR-negative but scattered HLA-DR-positive cells were observed within the epithelium and within the lamina propria (Fig. 5). Within the epithelium the distribution of HLA-DR-positive cells was similar to that of the $\mathrm{T}$ lymphocytes and this suggested that these HLA-DR-positive cells were T lymphocytes. On the other hand, the HLA-DR-positive cells detected in the lamina had a dendritic morphology.

\section{Discussion}

Using monoclonal antibodies in an indirect immunoperoxidase technique, we have identified lymphocyte subsets and macrophages in normal ureteric and bladder urothelium. Lymphocytes were located within the epithelium as well as in the subepithelial connective tissues and were almost always T cells with a preponderance of the suppressor/cytotoxic phenotype. The lymphocytes were concentrated within the epithelium, where $81.3 \%$ of the intra-epithelial $\mathrm{T}$ lymphocytes were of the suppressor/cytotoxic phenotype.

The pattern of lymphocyte distribution observed in this study is very similar to that noted in our earlier studies on normal human testis, epididymis, vas deferens, prostate and seminal vesicles (Ritchie et al., 1984; El-Demiry et al., 1986) where it was shown that approximately $70 \%$ of the T lymphocytes found within the mucosa of the male genital tract were of the suppressor/cytotoxic phenotype. Selby et al. (1981) showed that $79 \%$ of the intraepithelial $\mathrm{T}$ lymphocytes in the intestinal mucosa 

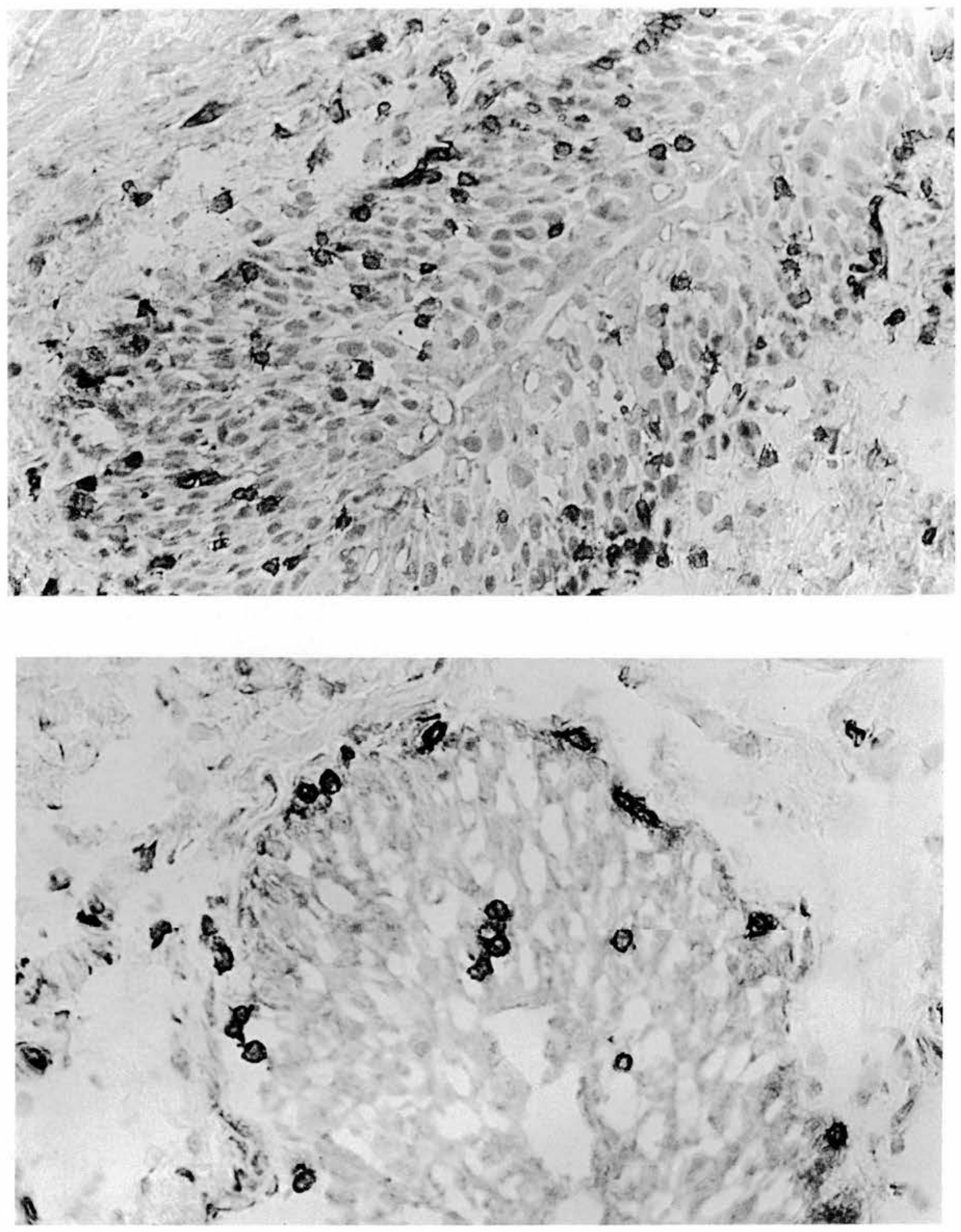

Figs 3A and B T8-positive cells (suppressor/cytotoxic T lymphocytes) within the epithelium and lamina propria of normal human ureter. (Cryostat section: immunoperoxidase $\times 160$ ). 
were also of the suppressor/cytotoxic phenotype. These findings suggest a common pattern of deployment of immune competent cells within mucosal surfaces and perhaps a shared mucosal immunity system. The urothelium has a wide surface area on which there is an interface between the environment and the tissues and although contamination with micro-organisms is frequent, frank infections are rare. Similarly, urine may contain numerous carcinogenic agents and yet urothelial malignancy is limited.

The presence of $\mathrm{T}$ lymphocyte subsets within the urothelium and within the lamina propria may imply that immune-related mechanisms may be directly involved in protection of the ureter and the bladder mucosa. The obvious preponderance of $T$ cells, macrophages and cells expressing HLA-DR antigens, combined with the paucity of B cells, suggests that cell-mediated immunity is more important than humoral immunity in effecting a local mucosal resistance. However, it is not clear whether these demonstrable T8-positive cells are functioning in a suppressor or a cytotoxic role, since both lymphocyte subsets are detected by this reagent. If indeed these lymphocytes were predominantly of the suppressor phenotype it might be argued that their function within the urothelium was to protect the epithelial micro-environment from immune-mediated damage. If, however, the T8-positive cells were mainly of the cytotoxic phenotype, then they would be of value both in resistance against certain infections and in tumour surveillance. Cytotoxic $\mathrm{T}$ cell mediated immune reactions could lead to the removal of damaged cells as well as urothelial cells with adherent bac-

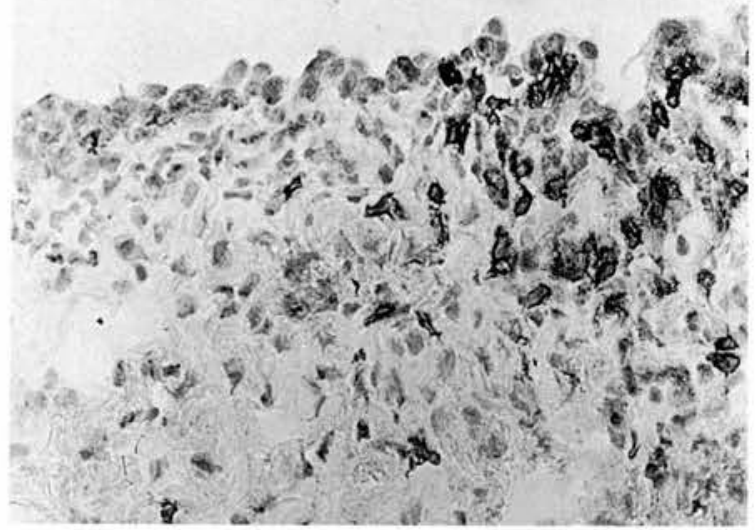

Fig. 4 T8-positive cells (suppressor/cytotoxic T lymphocytes) in normal bladder mucosa. (Cryostat section: immunoperoxidase $\times 160)$
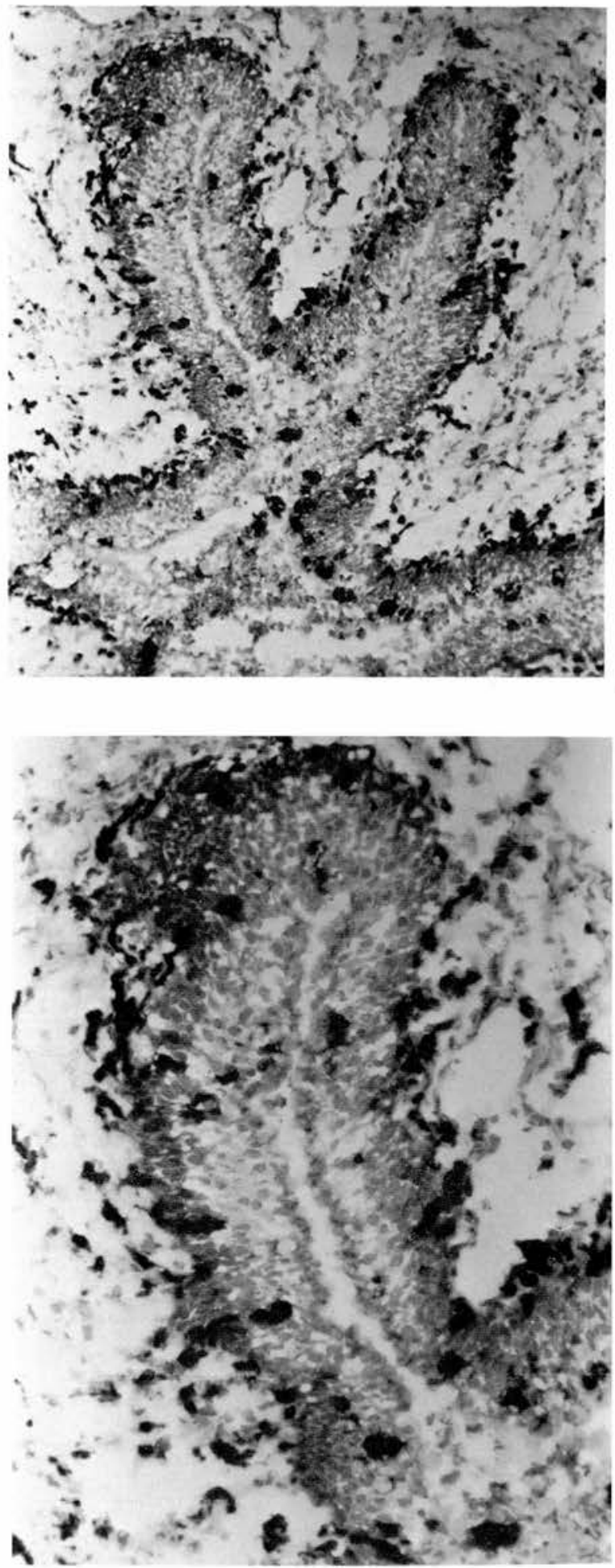

Figs 5A and B HLA-DR-positive cells within the epithelium and lamina propria of normal human ureter. (Cryostat section: immunoperoxidase $\mathrm{A} \times 40$; $\mathrm{B} \times 60$ ). 
teria, infected cells and cells damaged by environmental chemicals. It has been suggested that when an agent destroys a group of intestinal mucosal epithelial cells, local release of cytotoxic cells would provide a first line of cell-mediated defence of the de-epithelialised area, with macrophages further supplementing this initial action (Mowat and Ferguson, 1981). However, this matter will not be resolved until monoclonal antibodies which distinguish $\mathrm{T}$ suppressor from $\mathrm{T}$ cytotoxic lymphocytes are available and functional studies are performed on lymphocytes from the urothelium.

The presence of macrophages (Leu M3 + cells) within the mucosa of the ureter and the urinary bladder suggests that they also have a role in local mucosal immunity. They could perform a direct cytotoxic and/or phagocytic function against the invading micro-organisms or against aberrant cells and could also participate in the complex cellular interactions known to occur in both cellular and humoral immune responses (Friedman et al., 1981). In this context it is interesting to note the presence within the urothelium of cells possessing cell surface class II (Ia Dr-like) antigens which are thought to be essential for the successful presentation of antigen to T cells (Kappler and Marrack, 1976). This finding also suggests that cell-mediated immunity is more important than humoral immunity in the mucosa.

On the strength of these baseline findings in normal subjects, this work will be followed up by a study of mucosa from chronically inflamed and from neoplastic urothelia.

In conclusion, using specific monoclonal antibodies, $\mathrm{T}$ lymphocyte subsets and macrophages were identified in normal urothelium and shown to have a specific pattern of distribution. The existence of these cell populations may contribute to the health and protection of the urothelium, particularly in resistance to infection and tumour surveillance. These cell populations should also be considered in relation to the understanding of mucosal immunity in general.

\section{References}

El-Demiry, M. I. M., Hargreave, T. B., Busuttil, A., James, K., Ritchie, A. W. S. and Chisholm, G. D. (1985). Lymphocyte sub-populations in the male genital tract. British Journal of Urology, 57, 769-774.
El-Demiry, M. I. M., Hargreave, T. B., Smith, G., Busuttil, A., James, K and Chisholm, G. D. (1986). T-lymphocyte subsets in the human epididymis. In Proceedings of the Third International Forum of Andrology, Paris. (In press).

Friedman, S. M., Hunter, S. B., Irigoyen, O. H., Kung, P. C., Goldstein, G. and Chess, R. (1981). Functional analysis of human T-cell subsets defined by monoclonal antibodies. II. Collaborative T-T interactions in the generation of TNPaltered self-reactive cytotoxic T-lymphocytes. Journal of Immunology, 126, 1702-1705.

Kappler, J. W. and Marack, P. C. (1976). Helper T-cells recognise antigen and macrophage surface components simultaneously. Nature, 262, 797-798.

Mowat, A. M. and Ferguson, A. (1981). Induction and expression of mucosal cell-mediated immunity. In The Mucosal Immune System, ed. Bourne, F. J. Pp. 107-129. The Hague, Boston, London: Mertinus Nij Loff.

Poppema, S., Bhan, A. K., Reinherze, E. L., McCluskey, R. T. and Schlossman, S. F. (1981). Distribution of T-cell subsets in human lymph nodes. Journal of Experimental Medicine, 153, $30-41$.

Reinherz, E. L., Morimoto, C., Penta, A. C. and Schlossman, S. F. (1980). Regulation of B-cell immunoglobulin secretion by functional subsets of T-lymphocytes in man. European Journal of Immunology, 10, 570-572.

Reinherz, E. L. and Schlossman, S. F. (1980). The differentiation and function of human T-lymphocytes. Cell, 19, 821-827.

Ritchie, A. W. S., Hargreave, T. B., James, K. and Chisholm, G. D. (1984). Intra-epithelial lymphocytes in the normal epididymis. A mechanism for tolerance to sperm auto-antigens. British Journal of Urology, 56, 79-83.

Ritchie, A. W. S., James, K. and Micklem, H. S. (1983). The distribution and possible significance of cells identified in human lymphoid tissue by monoclonal antibody HNK-1. Clinical and Experimental Immunology, 51, 439-447.

Robertson, M. (1985). T-cell receptor, the present state of recognition. Nature, 317, 768-769.

Selby, W. S., Janossy, G., Goldstein, G. and Jewell, J. P. (1981). T-lymphocyte subsets in human intestinal mucosa: the distribution and relationship to $\mathrm{MHC}$-derived antigens. Clinical and Experimental Immunology, 44, 453-458.

\section{The Authors}

M. I. M. El-Demiry, MB, BCh(Cairo), MSc(Urol Cairo), Senior Resident in Urology, Police Hospital, Cairo, Egypt. Honorary Registrar, University Department of Surgery/Urology, Edinburgh.

T. B. Hargreave, MS, FRCS, FRCSE, Senior Lecturer and Consultant Urologist, University Department of Surgery/ Urology,

A. Busuttil, MD, MRCPath, Consultant Pathologist, Western General Hospital.

K. James, DSc, FRCPath, FRSE, Reader in Surgery, University of Edinburgh.

G. D. Chisholm, ChM, FRCS, FRCSE, Professor of Surgery and Consultant Urologist, Western General Hospital.

Requests for reprints to: M. I. M. El-Demiry, University Department of Surgery/Urology, Western General Hospital, Edinburgh EH4 2XU. 


\title{
Leucocytes in the Ejaculate from Fertile and Infertile Men
}

\author{
M. I. M. EL-DEMIRY, H. YOUNG, R. A. ELTON, T. B. HARGREAVE, K. JAMES and G. D. \\ CHISHOLM
}

\begin{abstract}
University Department of Surgery/Urology, Western General Hospital; Department of Bacteriology and Medical Computing and Statistics Unit, University Medical School, Edinburgh
\end{abstract}

\begin{abstract}
Summary-The presence of leucocytes and their subpopulations was studied in the ejaculate from 69 men with an infertile marriage and 12 fertile men. Monoclonal antibodies specific for human leucocytes were used in an immunoperoxidase technique. In addition to the standard sperm analysis, each specimen was also tested for sperm antibodies, aerobic and anaerobic microorganisms, Ureaplasma urealyticum and Chlamydia trachomatis. Leucocytes were found in large numbers in the fertile men compared with the patients. Lymphocytes were found in $20 \%$ of the patients. Micro-organisms were cultured from a similar proportion of both groups. We found no correlation between leucocyte counts, sperm density and motility, sperm antibodies and growth of micro-organisms. Our results cast doubt on the conventional criteria of subclinical genital tract infection, namely positive culture and excess leucocyte counts.
\end{abstract}

The role of subclinical genital tract infection in male infertility is controversial. Some studies have shown no difference in the results of culture for micro-organisms from semen samples from fertile and infertile populations and no correlation between sperm measurements (motility and density) and bacteriology. Comparing semen characteristics of fertile and infertile groups, McGowan et al. (1981) found that infection in the semen did not significantly affect the count, motility or volume of the specimen. Comhaire et al. (1980) and Naessens et al. (1986) also found no significant differences in the semen motility and density of infertile males with and without infection.

Others believe that there is a substantial proportion of infertile men with very minor symptoms but significant infection. Eliasson et al. (1967) showed that approximately $40 \%$ of infertile men in Sweden had cytological or bacteriological findings indicating infection or inflammation of the accessory

Read at the Combined Meeting of the British Association of Urological Surgeons and The Canadian Urological Association in London, June 1986 genital glands. They suggested that asymptomatic genital infection may be a major cause of infertility (Eliasson and Johannisson, 1978). Queseda et al. (1968), Moberg et al. (1980) and Swenson et al. (1980) also reported that infection of the semen may reduce sperm motility and density and hence affect fertility. There are two important reasons for this controversy. The first is a lack of agreement about criteria to diagnose subclinical genital tract infection and the second is that in many studies there has been a failure to culture the complete range of pathogenic micro-organisms; in most cultures Chlamydia has been omitted.

Traditional diagnosis is made by finding an excess of leucocytes in the ejaculate. However, it is difficult to distinguish leucocytes from the immature sperm cell using current techniques, which have many limitations and are not specific. In view of these problems, this study was undertaken to try and improve the diagnosis of infection by the use of a new technique to stain leucocytes. We have also undertaken culture from the same ejaculate for aerobic and anaerobic organisms, Chlamydia and genital Mycoplasma. 


\section{Patients and Methods}

The study was carried out on 81 men, 69 of whom were attending the infertility clinic because of an infertile marriage. The remainder were fertile men attending for vasectomy. The fertile men had fathered at least two children and at the time of testing had one child under 2 years of age. All were given clearly written instructions about the production and delivery of the semen specimens. In addition, a full medical history was obtained and a complete physical examination was performed.

\section{Semen}

Semen was collected by masturbation into sterile plastic containers after 3 days of sexual abstinence. Using sterile precautions, two aliquots were extracted from each specimen, one for standard semen analysis and the other for cultures for microorganisms. The remainder of the specimen was prepared for immunocytochemical staining for leucocytes. Semen analysis was performed as described in the World Health Organisation (WHO) Laboratory Manual (Belsey et al., 1980). Antisperm antibody measurement was done using the tray agglutination test (TAT) (Friberg, 1974) and the MAR test (Jager et al., 1978).

\section{Microbiological screening}

Bacterial cultures were done for aerobic and anaerobic organisms, Chlamydia trachomatis and Ureaplasma urealyticum. The methods have been described elsewhere (Hargreave et al., 1982; Young et al., 1983). All micro-organisms (aerobes, anaerobes, Ureaplasma urealyticum and Chlamydia trachomatis) were identified and reported separately (Taylor-Robinson et al., 1971; Duerden et al., 1980).

\section{Seminal leucocyte staining}

1. Preparation of a single cell suspension of the ejaculate. $1 \mathrm{ml}$ of the freshly collected ejaculate was mixed with $9 \mathrm{ml}$ Hanks Balanced Salt Solution (HBSS), calcium- and magnesium-free, in a $10 \mathrm{ml}$ centrifuge tube and centrifuged at $200 \times \mathrm{g}$ for $10 \mathrm{~min}$ at room temperature. The supernatant was decanted and the cell pellet was resuspended in $10 \mathrm{ml} \mathrm{HBSS}$ and centrifuged again at $200 \times \mathrm{g}$ for $10 \mathrm{~min}$. The cell pellet was then resuspended at a concentration of 5 to 10 million sperm $/ \mathrm{ml}$ in HBSS.

This procedure removed seminal plasma proteins and resulted in minimal cell loss and damge.

2. Preparation of cytocentrifuge smears of washed ejaculate. $100 \mu \mathrm{l}$ of the cell suspension were put in each cytospin block and spun down on to alcoholcleaned glass slides using a Shandon Cytocentrifuge (Shandon-Elliot, Southern Instruments Ltd). This was achieved by spinning at $800 \mathrm{rpm}$ for $10 \mathrm{~min}$. The smears were circled with a diamond marker, air dried for $1 \mathrm{~h}$ and then fixed in acetone for $10 \mathrm{~min}$ at room temperature. The smears were either stained immediately or stored, wrapped in aluminium foil and kept at $-20^{\circ} \mathrm{C}$ until used.

3. Immunoperoxidase staining. The indirect immunoperoxidase technique was used throughout. The procedure has been fully described by El-Demiry et al. (1986). The essential steps were as follows. Smears were incubated with the different antihuman leucocyte monoclonal antibodies, followed by peroxidase conjugated rabbit anti-mouse antibody. Full details of the antibodies used are given in Table 1. In the case of negative controls the primary antibodies were omitted. As positive controls the monoclonal antibodies were applied to cytocentrifuged smears of human peripheral blood leucocytes. The colour reaction was developed by incubating the smears with diaminobenzidinehydrogen peroxide substrate. Smears were finally counter-stained with haematoxylin, dehydrated and mounted in DPX.

Smears were examined by light microscopy (magnification $\times 320$ ). The positively labelled cells were identified by their dark brown cell membrane staining (Figs 1 and 2). Stained cells with any individual monoclonal antibody were counted in 10 different fields for each smear. The average was taken and multiplied by the dilution (the number of $\mathrm{ml}$ in which the pellet was resuspended).

The resulting numbers of leucocytes reported in the Tables have no independent meaning but they allow comparison between patients and distinction of those with high or low leucocyte numbers.

Table 1 *Monoclonal Antibodies Reacting with Human Leucocytes

\begin{tabular}{ll}
\hline $\begin{array}{l}\text { Monoclonal antibody } \\
\begin{array}{l}\text { Anti-human leucocytes } \\
\text { (Hle-1) }\end{array}\end{array}$ & All leucocytes \\
Anti-Leu 4 & All T lymphocytes \\
Anti-Leu 3a & Helper/inducer T lymphocytes \\
Anti-Leu 2a & $\begin{array}{l}\text { Suppressor/cytotoxic T } \\
\text { lymphocytes }\end{array}$ \\
Anti-Leu 12 & B lymphocytes \\
\hline
\end{tabular}

*These monoclonal antibodies were obtained from Becton/ Dickenson, Lab Impex Limited, Twickenham. 


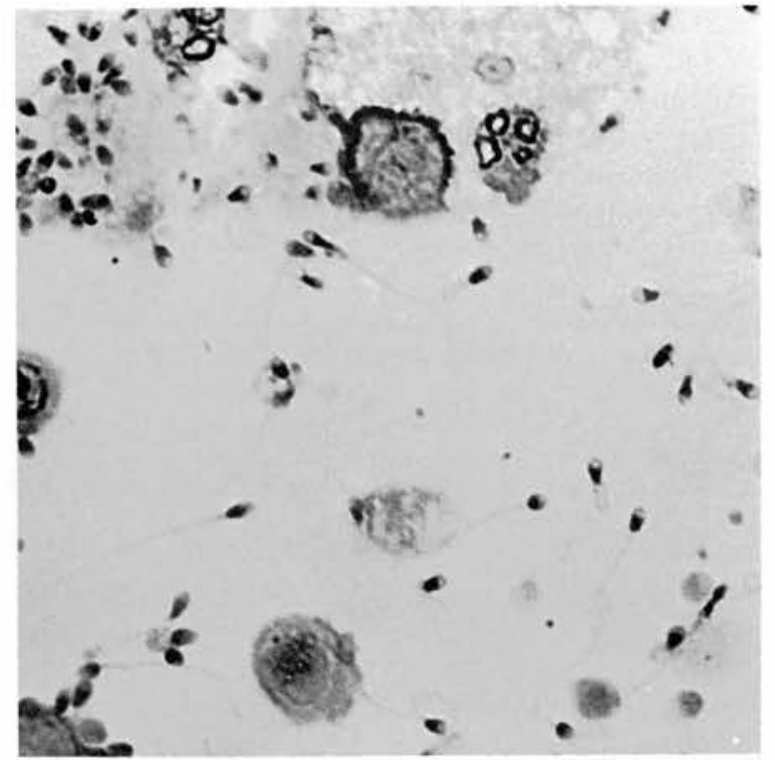

Fig. 1 Immunoperoxidase staining of cytocentrifuge smear of semen with antihuman leucocyte (Hle-1) monoclonal antibody. $(\times 320)$.

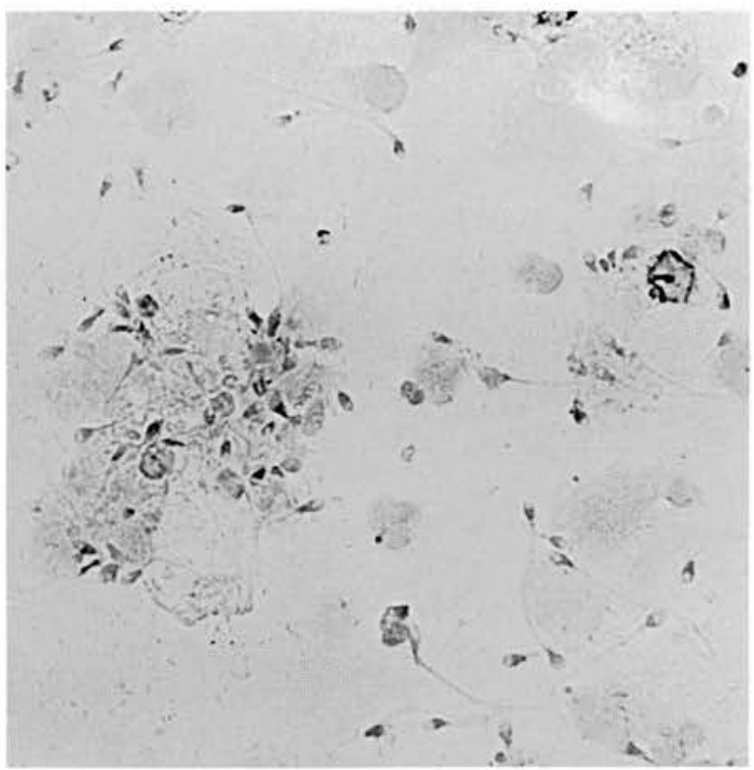

Fig. 2 Leu $2 \mathrm{a}+$ cells (suppressor/cototoxic $\mathrm{T}$ lymphocytes) in semen stained with anti-Leu $2 \mathrm{a}$ monoclonal antibody. (Immunoperoxidase $\times 320$ ).

\section{Statistical methods}

Between-group comparisons of leucocyte and bacterial species and counts were made using Wilcoxon rank sum tests, and the significance of relationships between these measurements was assessed by Kendal rank correlation co-efficients. Relationships between leucocyte or bacterial counts and subsequent fertility were tested by couple-months analysis (Hargreave and Elton, 1983).

\section{Results}

\section{Microbiological investigations}

Sixty-five patients and eight fertile men were tested for the following micro-organisms: aerobes, anaerobes, Ureaplasma urealyticum and Chlamydia trachomatis: 57 of the 65 patients and 7 of the 8 fertile men were found to have positive bacterial cultures for one or more of these micro-organisms (Table 2). Eight patients and one fertile man had completely negative results. There were no positive cultures for Chlamydia.

Aerobic organisms were isolated either alone or in association with anaerobes or Ureaplasma from 55 patients and seven fertile men. Anaerobes were isolated from 43 patients and from all of the fertile men with positive cultures. Nine patients and one fertile man had positive culture for Ureaplasma urealyticum.

There were no significant differences in bacterial counts among individual organisms or groups except for Proteus, which was significantly more common in the fertile men (2/8) compared with the patients $(2 / 65)$. The most frequent organism in both patients and fertile men was Staphylococcus albus (Table 3) with a mean (range) count of colony forming units isolated of $1.1 \times 10^{5}\left(1.5 \times 10^{2}-1.5 \times\right.$ $10^{6}$ ). Correlating the bacterial count of every individual organism detected with sperm density and motility, the results were not significant except

Table 2 Number of Bacterial Species* Isolated per Semen Sample

\begin{tabular}{|c|c|c|}
\hline \multirow[t]{2}{*}{$\begin{array}{l}\text { No. of bacterial species } \\
\text { isolated per patient }\end{array}$} & \multicolumn{2}{|c|}{$\begin{array}{l}\text { No. of patients with } \\
\text { corresponding number of } \\
\text { species (and total number } \\
\text { of isolates) }\end{array}$} \\
\hline & Infertile patients & Controls \\
\hline 0 & $8(0)$ & $1(0)$ \\
\hline 1 & $12(12)$ & $3(3)$ \\
\hline 2 & $11(22)$ & $1(2)$ \\
\hline 3 & $17(51)$ & $3(9)$ \\
\hline 4 & $12(48)$ & $0(0)$ \\
\hline 5 & $3(15)$ & $0(0)$ \\
\hline 6 & $2(12)$ & $0(0)$ \\
\hline Total & $65(160)$ & $8(14)$ \\
\hline
\end{tabular}

* Excluding Ureaplasma urealyticum

Note: Mean number of organisms per control sample $=1.75(14 /$ 8). Mean number of organisms per infertile sample $=2.46(160 /$ 65 ). None of the controls had $>3$ bacterial species present per sample. 
Table 3 Isolation Rate of Bacteria from 73 Semen Samples

\begin{tabular}{lll}
\hline & $\begin{array}{l}\text { Number (and percentage) of } \\
\text { samples with corresponding } \\
\text { organism(s) }\end{array}$ \\
\cline { 2 - 3 } & $\begin{array}{l}\text { Infertile patients } \\
(n=65)\end{array}$ & $\begin{array}{l}\text { Controls } \\
(n=8)\end{array}$ \\
Species group & $48(73.8)$ & $5(62.5)$ \\
Staphylococcus albus & $27(41.5)$ & $3(37.5)$ \\
Diphtheroids & $10(15.4)$ & 0 \\
haemolytic streptococci & $1(1.5)$ & 0 \\
Streptococcus faecalis & $1(1.5)$ & $1(12.5)$ \\
Proteus spp & $2(3.1)$ & $2(25.0)$ \\
Escherichia coli & $3(4.6)$ & 0 \\
Bacteroides spp & $31(47.7)$ & $2(25.0)$ \\
(a) Anaerobic cocci & $31(47.7)$ & $1(12.5)$ \\
(b) Ureaplasma urealyticum & $9(13.9)$ & $2(25.0)$ \\
(c) Others & $6(9.2)$ & 0 \\
\hline
\end{tabular}

(a) Bacteroides and/or anaerobic cocci were isolated from 43 samples $(66.2 \%)$ from infertile patients and from 3 samples $(37.5 \%)$ from controls.

(b) Ureaplasma isolation rate based on 70 valid cultures.

(c) Includes Fusobacterium nucleatum 1; Eubacterium lentium 2; Proprionibacterium acnes 1; Gardnerella vaginalis 1; and Lactobacillus $\operatorname{spp} 1$.

for Bacteroides asaccharolyticus, which showed a negative correlation with density and motility $(P<0.05)$.

\section{Leucocytes in the semen}

Comparison of patients with an infertile marriage and fertile men. All of the specimens from the 12 fertile men showed cells reacting with the pan-antileucocyte monoclonal antibody with a mean count of $44.9 \pm 25.8$ leucocytes per high power field $(\times 320)$. Most of these cells appeared from their morphological appearances to be polymorphonuclear (PMN) leucocytes. No cells reacting with the panantilymphocyte reagent were detected.

Leucocytes were detected in $90 \%(63 / 67)$ of the specimens from the patients, with a mean count of $14.6 \pm 17.1$ leucocyte per HPF $(\times 320)$. T lymphocytes (Leu $4+$ ) with predominance of the suppressor/cytotoxic phenotype (Leu 2a+) (Fig. 2) were also demonstrated in 13 patients with a mean count of $4.5 \pm 3.3 \mathrm{~T}$ lymphocytes per HPF $(\times 320)$. The number of PMN leucocytes was calculated by subtracting the pan-lymphocyte count from the pan-leucocyte count. Wilcoxon rank sum tests showed that the total leucocyte count and the polymorphonuclear leucocytes were significantly higher in fertile men $(P<0.001$ in both), but there were no significant differences in the counts of $\mathrm{T}$ lymphocytes or their subsets.
Relationship of leucocytes to bacterial counts. Each individual bacterial count, Ureaplasma and bacterial groups were correlated with the leucocyte and leucocyte subpopulation counts. The results were not statistically significant (Tables 4,5 and 6) except that $\beta$-streptococci showed a significant positive correlation with T lymphocytes $(P<0.05)$.

Table 4 Relationship between Number of Bacterial Species per Semen Sample and Leucocyte Count

\begin{tabular}{lcccc}
\hline $\begin{array}{l}\text { No. of bacterial species } \\
\text { isolated per patient }\end{array}$ & $\begin{array}{l}\text { No. of patients with leucocyte } \\
\text { counts of }\end{array}$ & Total \\
\hline & $<1$ & $1-20$ & $>20$ & \\
0 & 1 & 3 & 2 & 6 \\
1 & 1 & 8 & 3 & 12 \\
2 & 4 & 6 & 1 & 11 \\
3 & 5 & 10 & 2 & 17 \\
4 & 2 & 8 & 2 & 12 \\
5 & 1 & 2 & 0 & 3 \\
6 & 0 & 1 & 1 & 2 \\
Total & 14 & 38 & 11 & 63 \\
\hline
\end{tabular}

Kendall rank correlation $=\mathrm{t}=0.01$ (not significant).

Table 5 Relationship between Presence of Anaerobes and Leucocyte Count

\begin{tabular}{llcl}
\hline $\begin{array}{l}\text { Leucocyte } \\
\text { count }\end{array}$ & $\begin{array}{l}\text { Anaerobes } \\
\text { present }\end{array}$ & $\begin{array}{l}\text { Anaerobes } \\
\text { absent }\end{array}$ & Total \\
\hline$<1$ & 12 & 2 & 14 \\
$1-20$ & 22 & 16 & 38 \\
$>20$ & 7 & 4 & 11 \\
Total & 41 & 22 & 63 \\
\hline
\end{tabular}

Wilcoxon rank sum test showed non-significant correlation $(\mathrm{z}=$ 1.07).

Table 6 Relationship between Ureaplasma Culture and Leucocyte Count in Infertile Men

\begin{tabular}{lll}
\hline & \multicolumn{2}{l}{ Ureaplasma culture } \\
\cline { 2 - 3 } & Positive & Negative \\
\cline { 2 - 3 } & $\begin{array}{l}\text { No. of patients } \\
(\% \text { of }+v e)\end{array}$ & $\begin{array}{l}\text { No. of patients } \\
(\% \text { of }- \text { ve })\end{array}$ \\
\hline$<1$ & $2(22.2)$ & $12(23)$ \\
$1-20$ & $5(55.5)$ & $30(57)$ \\
$>20$ & $2(22.2)$ & $11(21)$ \\
\hline
\end{tabular}

Relationship of leucocytes to antisperm antibodies. Serum TAT results were available for 50 patients and MAR IgG for 19. Of the correlations with leucocyte counts, only that between MAR IgG and PMN leucocytes was significant (positive $P<0.05$ ). 
However, there was no significant correlation with serum TAT.

Relationship of leucocytes to sperm density, motility and subsequent fertility. There was no correlation between the leucocyte count and the duration of involuntary infertility, sperm motility and sperm density (Kendal rank correlation of $-0.03,0.07$ and 0.05 respectively, NS). Furthermore, using the couple-months analysis method, there was no significant relationship between leucocyte counts and subsequent fertility (Table 7).

Table 7a Relationship of Total Leucocyte Counts to Subsequent Fertility

\begin{tabular}{lll}
\hline Pan leucocyte count & Observed pregnancies & $\begin{array}{l}\text { Expected } \\
\text { pregnancies }\end{array}$ \\
\hline 0 & 2 & 1.47 \\
$0<10$ & 2 & 3.94 \\
$10<20$ & 4 & 2.24 \\
$>20$ & 1 & 1.35 \\
\hline
\end{tabular}

$\chi^{2}=2.63 \mathrm{df}(\mathrm{NS})$

Table 7b Relationship of T lymphocytes to Subsequent Fertility

\begin{tabular}{lll}
\hline Tlymphocyte count & Observed pregnancies & $\begin{array}{l}\text { Expected } \\
\text { pregnancies }\end{array}$ \\
\hline 0 & 5 & 7.11 \\
$>0$ & 4 & 1.89 \\
\hline
\end{tabular}

$\chi^{2}=2.991 \mathrm{df}(\mathrm{NS})$

\section{Discussion}

In this study we tried to find a possible relationship between subclinical genital tract infection and male infertility. We defined precisely the leucocytes and leucocyte subpopulations in the ejaculate using specific monoclonal antibodies in an immunoperoxidase technique. We also performed cultures for a range of micro-organisms.

Leucocytes were detected in all of the fertile men (12) and in 63 of the 65 patients $(90 \%)$ with an infertile marriage. Polymorphonuclear leucocytes were the predominant cell type in both groups. The fertile men were found to have a significantly larger number of leucocytes (mainly PMN leucocytes) compared with the patients. T lymphocytes were detected in only 13 of the 63 patients. A high bacterial isolation rate was found: $87 \%$ of the semen cultures of both the fertile and infertile groups were positive for micro-organisms with no difference between the patients and fertile groups. However, there was no significant correlation between leucocyte counts and the counts of positive cultures for all micro-organisms. The significant correlation between $\beta$-streptococci and T lymphocytes $(P<0.05)$ is difficult to explain but may be a spurious result from multiple statistical tests. Furthermore, no correlations were found between leucocyte counts and sperm density, motility and subsequent fertility.

In 1981 Fowler reviewed the literature and found little evidence to support infection as a frequent cause of infertility.

Our results are also in agreement with those of other studies. Naessens et al. (1986) reported that $97.7 \%$ of the semen cultures were positive for aerobes and anaerobes and they found that no single aerobic or anaerobic organism could be related to abnormal semen samples. Comhaire et al. (1980) found no significant difference in semen characteristics of infertile males with and without infection. McGowan et al. (1981) found that infection in the semen did not significantly affect the count, motility or volume of the specimen. In his experience with 290 patients who had semen cultured, Hendry (1983) found that pregnancies were produced with roughly equal frequency with or without positive semen cultures irrespective of whether the organisms were treated with antibiotics or not.

Our observation that none of the controls and the infertile men was positive for Chlamydia may be due to the fact that all men examined had no history of specific or non-specific genital tract infections and Chlamydia is not a commensal organism. However, Ulstein et al. (1976) were unable to culture Chlamydia from the urine, expressed prostatic secretion or semen of 21 asymptomatic men with unexplained infertility.

Our finding that the fertile men had large numbers of polymorphonuclear leucocytes compared with the patients with an infertile marriage raises many questions about the normal role of these cells in the ejaculate. These cells are important in host defence against invading micro-organisms but are also able to destroy tissue cells. It is possible that these polymorphonuclear leucocytes have a role in the phagocytosis of the residues of degenerating germ cells and of residual bodies in the ejaculate. Another possibility is that they play a role in the destruction and removal of the dead and/ or immature sperm. In this connection it is interesting to note that leucocyte phagocytosis of 
antibody coated sperm has been demonstrated by London et al. (1985).

In conclusion, the evidence from both the leucocyte and the bacterial studies suggests that subclinical genital tract infection in asymptomatic patients has no major role in male infertility. We found no correlation between sperm density, sperm motility, the presence of antisperm antibodies, positive bacterial cultures and the leucocyte response in the semen. Our results cast doubts on the currently accepted criteria of subclinical genital tract infection.

\section{References}

Belsey, M. A., Eliasson, R., Gallegos, A. J., Morghissi, K. S., Paulsen, C. A. and Praval, M. R. N. (eds). (1980). WHO Laboratory Manual for the Examination of Human Semen and Cervical Interaction. Pp. 9-13. Distributed by Special Program Research Development and Research Training in Human Reproduction. Geneva: World Health Organisation.

Comhaire, E., Verschreagen, G. and Vermeulen, L. (1980). Diagnosis of accessory gland infection and its possible role in male infertility. International Journal of Andrology, 3, 32-45.

Duerden, B. I., Collee, J. G., Brown, R., Deacon, A. G. and Halbrook, W. P. (1980). A scheme for the identification of clinical isolates of gram negative anaerobic bacilli by conventional bacteriological tests. Journal of Medical Microbiology, 13, 231-245.

El-Demiry, M. I. M., Hargreave, T. B., Busuttil, A., James, K. and Chisholm, G. D. (1986). Identifying leucocytes and leucocyte subpopulations in semen using monoclonal antibody probes. Urology. (In press).

Eliasson, R., Fredricsson, B., Johannisson, E. and Leander, G. (1967). Biochemical and morphological changes in semen from men with diseases in the accessory genital glands. Excerpta Medica International Congress Series, 133, 625.

Eliasson, R. and Johannisson, E. (1978). Cytological studies of prostatic fluids from men with and without abnormal palpatory findings of the prostate. II. Clinical application. International Journal of Andrology, 1, 582-588.

Fowler, J. E. (1981). Infections of the male reproductive tract and infertility: a selected review. Journal of Andrology, 3, 121131.

Friberg, J. (1974). A simple and sensitive micromethod for demonstration of sperm agglutinating activity in serum from infertile men and women. Acta Obstetricia et Gynecologica Scandinavica Supplement (Stockholm), 36, 21-29.

Hargreave, T. B. and Elton, R. A. (1983). Is conventional sperm analysis of any use? British Journal of Urology, 55, 774-779.

Hargreave, T. B., Torrance, M., Young, H. and Harris, A. B. (1982). Isolation of Ureaplasma urealyticum from seminal plasma in relation to sperm antibody levels and sperm motility. Andrologia, 14, 223-227.

Hendry, W. F. (1983). Treatment of antisperm antibodies. In Male Infertility, ed. Hargreave, T. B. Chapter 16, pp. 280 296. Berlin, Heidelberg, New York, Tokyo: Springer-Verlag.

Jager, S., Kremer, J. and Van Slochteren-Draaisma, T. (1978). A simple method of screening for antisperm antibodies in the human male. Detection of spermatozoal surface IgG with the direct mixed antiglobulin reaction carried out on untreated fresh human semen. International Journal of Fertility, 23, 1221.

London, S. N., Haney, A. I. and Weinberg, J. B. (1985). Macrophages and infertility: enhancement of human macrophage mediated sperm killing by antisperm antibody. Fertility and Sterility, 43, 274-278.

McGowan, M. P., Burger, H. G., Baker, H. W. G., de Kester, D. M. and Kovacs, G. (1981). The incidence of non-specific infection in the semen in fertile and subfertile males. International Journal of Andrology, 4, 657-662.

Moberg, P. J., Eneroth, P., Ljung, A. and Nord, C. (1980). Bacterial flora in semen before and after doxycycline treatment in infertile couples. International Journal of Andrology, 3, 4558.

Naessens, A., Foulon, W., Debrucker, P., Devroey, P. and Lanwers, S. (1986). Recovery of micro-organisms in semen and relationship to semen evaluation. Fertility and Sterility. 45, 101-105.

Queseda, E. M., Dukes, G. D., Deen, G. H. and Franklin, R. R. (1968). Genital infection and sperm agglutinating antibodies in infertile men. Journal of Urology, 19, 106-108.

Swenson, C. E., Toth, A., Toth, C., Wolfgruber, L. and O'Leary, W. M. (1980). Asymptomatic bacteriospermia in infertile men. Andrologia, 12, 7-11.

Taylor-Robinson, D., Martin-Bouron, C., Watanbe, T. and Addey, J. P. (1971). Isolation of T-mycoplasmas from dogs and squirrel monkeys, biological and serological comparison with those isolated from man and cattle. Journal of General Microbiology, 68, 97-107.

Ulstein, M., Capell, P., Holmes, K. K. and Paulsen, C. A. (1976). Non-symptomatic genital tract infection and male infertility. In Human Semen and Fertility Regulation in Men, ed. Hafez, E. S. E. Pp. 355-362. St Louis: Mosby.

Young, H., Sarafian, S. K., Harris, A. B. and McMillan, A. (1983). Non-cultural detection of Neisseria gonorrhoeae in cervical and vaginal washings. Journal of Medical Microbiology, 16, 183-191.

\section{The Authors}

M. I. M. El-Demiry, MB, BCh(Cairo), MSc(Urol, Cairo), Senior Resident in Urology, Police Hospital, Cairo, Egypt; Honorary Registrar, University Department of Surgery/Urology.

H. Young, BSc, PhD, MRCPath, Lecturer, Department of Bacteriology, University of Edinburgh.

R. A. Elton, PhD, Lecturer, Medical Computing and Statistics Unit, University of Edinburgh.

T. B. Hargreave, MS, FRCS, FRCSE, Senior Lecturer and Consultant Urologist, University Department of Surgery/ Urology.

K. James, DSc, FRCPath, FRSE, Reader in Surgery, University of Edinburgh.

G. D. Chisholm, ChM, FRCS, FRCSE, Professor of Surgery and Consultant Urologist, Western General Hospital.

Requests for reprints to: M. I. M. El-Demiry, University Department of Surgery/Urology, Western General Hospital, Edinburgh EH4 2XU. 


\title{
IDENTIFYING LEUCOCYTES AND LEUCOCYTE SUBPOPULATIONS IN SEMEN USING MONOCLONAL ANTIBODY PROBES
}

\author{
M. I. M. EL-DEMIRY, M.B., B.Ch., M.Sc. (Urol.) \\ T. B. HARGREAVE, M.S., F.R.C.S. \\ A. BUSUTTIL, M.D. \\ K. JAMES, F.R.C. PATH., F.R.S.E. \\ G. D. CHISHOLM, CH.M., F.R.C.S. \\ From the University Department of Surgery/Urology, and \\ Department of Pathology, Western General Hospital, \\ Edinburgh, Scotland
}

\begin{abstract}
An indirect immunoperoxidase technique employing specific monoclonal antibodies has been used to identify leucocyte subpopulations in cytocentrifuge smears of washed human ejaculate. Cells reacting with the pan antihuman leucocyte monoconal antibody (HLe-1) were demonstrated in 63/67 specimens from subfertile patients with a mean count of $14.5 \pm 17.1$ leucocytes per $H P F(\times 320)$. Cells with similar reactivity were observed in all specimens examined from 10 fertile men with a mean count of $41.6 \pm 26.3$ leucocytes per HPF $(\times 320)$. Leu-T4 + cells (T-lymphocytes) were demonstrated in only $13 / 63$ of the subfertile group with a mean count of $4.46 \pm 3.3 \mathrm{~T}$ lymphocytes per HPF $(\times 320)$. Studies with the anti-leu $2 a$ antibody revealed that these leu- $4+$ cells were mainly of the suppressor/cytotoxic phenotype. In contrast, no leu-4 + cells were detected in the control group. No leu-12 + cells (B-lymphocytes) were detected in any of the 80 specimens examined.
\end{abstract}

The role of subclinical genital infection in male infertility remains controversial. Male genital tract infection may be difficult to diagnose due to the paucity of symptoms and the lack of clear clinical, bacteriologic, and seminal fluid criteria. The mere presence of bacteria in the seminal plasma is an inadequate criterion for the diagnosis of infection. ${ }^{1}$

The importance, and upper limit of normality, of white cells in the semen as well as the prostate vesicular fluid have not been clearly established with respect to fertility. ${ }^{2,3}$ Also several types of immature germ cells are found in semen including spermatids, spermatocytes, and even spermatogonia, ${ }^{4}$ and these sperm precursors may be confused with white blood cells when ordinary staining techniques are used. Thus early nonseparated spermatids that share a common cytoplasm may be mistaken for polymorph nuclear leucocytes (PMN), while round spermatids (Sa-b) and occasionally spermatocytes may be mistaken for lymphocytes. Because of these problems new techniques have been developed to facilitate discrimination between white blood cells and immature germ cells. For example, Endtz ${ }^{5}$ has applied a histochemical technique based on the use of benzedine for staining leucocytes while Nahoum and Cardozo $^{6}$ have proposed the use of O-toluidine as a substitute for benzedine for histochemical staining of these cells. Couture et al. ${ }^{7}$ have used a combination of Bryan's ${ }^{8}$ sperm stain, which 
reacts with acrosomal cap of the spermatozoa and the spermatids, and Leishman's blood stain which interacts with leucocytes. ${ }^{9}$

These techniques which depend mainly on the endogenous peroxidase activity of the leucocytes have certain limitations. Lymphocytes do not show peroxidase activity at any stage of maturation, basophils do not stain, and monocytes show faint diffuse cytoplasmic staining. ${ }^{10}$ Furthermore PMN leucocytes peroxidase activity depends on the age and condition of the cell and on the freshness of the smear at the time of staining. ${ }^{11}$ Thus older PMN leucocytes and old smears may not exhibit this granular staining. Peroxidase activity also is unstable in light. Also peroxidase reaction may be negative in the neutrophils which appear in the peripheral blood in response to severe infection, ${ }^{10}$ and this may also occur in the semen.

The attempt of Couture et al. ${ }^{7}$ to differentiate lymphocytes from Sa-b spermatids using Bryan's sperm stain ${ }^{8}$ for the acrosomal cap is also unreliable because the acrosome may not be fully developed and is difficult to demonstrate by light microscopy. ${ }^{12}$ Also in abnormal semen there are many aberrations, and the number of immature spermatids with loosened or missing acrosomes may increase.

In an attempt to overcome these limitations in this study, we have used antihuman leucocytes monoclonal antibodies in an immunocytochemical technique to define precisely the leucocytes and their subpopulations in semen.

\section{Material and Methods}

Semen specimens were obtained from 80 men, 70 of which were subfertile. The remainder were from fertile men attending for vasectomy. The fertile men had fathered at least two children, and at the time of testing had one child under two years of age. All were given clearly written instructions about the production and delivery of the specimens. Using sterile precautions, 2 aliquots were extracted from each specimen, one for standard semen analysis and Papanicolaou staining and the other for microbiologic cultures. The remainder of the specimen was prepared for immunocytochemical staining.

\section{Monoclonal antibodies}

A series of monoclonal antibodies that react with human leucocytes and leucocyte subpopulations (The Becton-Dickinson Series) were obtained from Lab Impex Limited (Twick-
TABLE I. Monoclonal antibodies reacting with human leucocytes

\begin{tabular}{ll}
\hline Monoclonal Antibody & \multicolumn{1}{c}{ Specificity } \\
\hline $\begin{array}{c}\text { Anti-human leucocytes } \\
\text { (HLe-1) }\end{array}$ & All leucocytes \\
Anti-leu 4 & All T-lymphocytes \\
Anti-leu 3a & Helper/inducer \\
& T-lymphocytes \\
Anti-leu 2a & Suppressor/cytotoxic \\
Anti-leu 12 & T-lymphocytes \\
\hline
\end{tabular}

enham, Middlesex). Further details of these reagents are listed in Table I. The specifications of these antibodies have been described previously. ${ }^{13-15}$

\section{Immunoperoxidase staining of cytocentrifuged smears of semen}

Cytocentrifuge smears were prepared as follows:

1. One $\mathrm{ml}$ of ejaculate was mixed with $24 \mathrm{ml}$ of Hank's balanced salt solution in a $25-\mathrm{ml}$ universal tube and centrifuged at $200 \times \mathrm{g}$ for ten minutes. This procedure, which removed seminal plasma proteins, resulted in minimal loss and damage of cells.

2. The supernatant was aspirated, and the cell pellet was resuspended at a concentration of 5-10 million spermatozoa per $\mathrm{ml}$ in Hank's solution and spun down on to clean glass slides (100 $\mu \mathrm{l}$ each) using a Shandon cytocentrifuge (Shandon-Elliot, Cheshire, UK). This was done by spinning at $800 \mathrm{rpm}$ for ten minutes.

3 . The smears were circled with a diamond marker, air dried for one hour, and then fixed in acetone for ten minutes after which they were either stained or wrapped in aluminum foil and stored at $-20^{\circ} \mathrm{C}$ until used.

\section{Staining procedures}

The indirect immunoperoxidase technique was used, as follows:

1. The primary monoclonal antibodies diluted 1:100 in tris-buffered saline (TBS) at $\mathrm{pH} 7.6$ were applied to the smears $(60 \mu \mathrm{l}$ each) except the negative control smears. The slides were incubated in humid chambers for thirty minutes at room temperature.

2. Following a five-minute wash in TBS, peroxidase conjugated rabbit antimouse Ig (Dakopatts, Copenhagen, Denmark) diluted $1: 30$ in TBS was applied to the smears $(60 \mu \mathrm{l}$ each). The slides were re-incubated for thirty minutes; then washed in TBS for five minutes. 


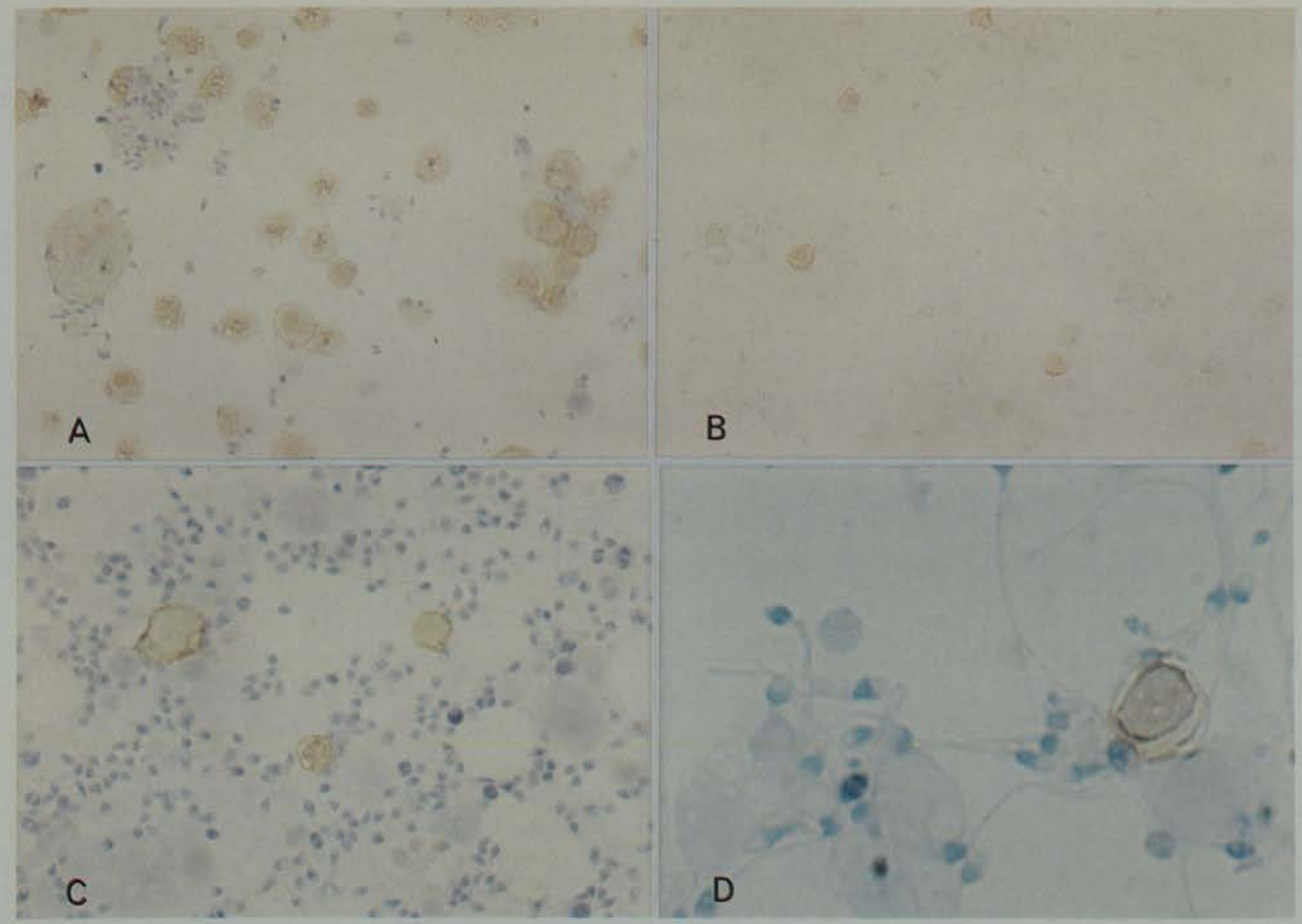

FIGURE 1. Immunoperoxidase staining of cytocentrifuge smear of semen with (A) antihuman leucocyte (HLe-1) monoclonal antibody, (B) anti-leu 4 monoclonal antibody (Pan T-lymphocyte marker), (C) leu-2a+ cells (suppressor/cytotoxic T-lymphocytes) stained with anti-leu $2 a$ monoclonal antibody, and (D) leu-3a+ cell (helper/inducer T-lymphocyte) stained with anti-leu 3 a monoclonal antibody. (Original magnifications $A, B$, and $C, \times 320 ;(D) \times 640$.)

(Normal human serum was added $1: 25 \mathrm{v} / \mathrm{v}$ to the conjugated antibody to block cross reactivity against human immunoglobulins.

3. The slides were finally incubated with freshly prepared diaminobenzidine $(0.6 \mathrm{mg} / \mathrm{ml})$ hydrogen peroxide $(0.01 \% \mathrm{v} / \mathrm{v})$ substrate for five minutes, washed in tap water and counterstained in Mayer's hematoxylin for thirty seconds.

4. The slides were washed once more in tap water and mounted in Apathy's aqueous mounting medium.

It should be stressed that (1) cytocentrifuge preparations of human peripheral blood leucocytes were used as positive controls throughout this study, and (2) phagocytic cells were identified by pre-incubating peripheral blood leucocytes with fluorescent-labeled latex beads for one hour.

\section{Immunologic analysis and scoring}

Smears were examined by light microscopy using a magnification of $\times 320$. The positively labeled cells were identified by their dark brown cell membrane staining. Stained cells with any individual monoclonal antibody were counted in ten different fields for each smear. The average was taken and multiplied by the dilution (number of mls in which the pellet was resuspended). The values expressed as the mean \pm standard deviation.

\section{Results}

\section{Staining pattern}

The staining pattern seen in semen smears was similar to that obtained in peripheral blood leucocyte smears. The positively stained cells were easily identified by their dark brown cell membrane staining (Fig. 1A). The majority of these stained cells appeared from their morphologic appearance to be PMN leucocytes. Cells actively involved in phagocytosis showed specific membrane staining. The distribution of the positively labeled cells was nearly uniform throughout the smears, and in any particular 
TABLE II. Leucocytes in semen detected by antihuman leucocyte (HLe-1) monoclonal antibody

\begin{tabular}{ccc}
\hline \multirow{2}{*}{$\begin{array}{c}\text { Leucocytes/HPF } \\
(\times 320)\end{array}$} & Patients & $\begin{array}{c}\text { Number of } \\
\text { Fertile Men } \\
\text { (Controls) }\end{array}$ \\
\hline 0 & 7 & 0 \\
$1-10$ & 35 & 0 \\
$11-20$ & 15 & 2 \\
$>20$ & 13 & 8 \\
\hline
\end{tabular}

specimen the count from one field to another was consistent. Few leucocytes showed yellowish brown peroxidase positive granules in their cytoplasm. Negative control smears showed no staining.

\section{Fertile men}

All the specimens from the 10 fertile men were found to contain cells reacting with the pan antileucocyte antibody (HLe-1). From their morphologic appearance, these positively stained cells appeared to be polymorphonuclear leucocytes. The mean count was $41.6 \pm 26.3$ leucocytes per high-power field $(\times 320)$. No cells reacting with anti- $T$ and anti-B cell reagents were detected.

\section{Patients}

Cells reacting with the antihuman leucocyte monoclonal antibody (HLe-1), which defines all leucocytes, were demonstrated in 63/67 $(90 \%)$ specimens with a mean count of $14.6 \pm$ 17.1 leucocytes/HPF $(\times 320)$ (Table II). Leu$4+$ cells (T-lymphocytes) were demonstrated in $13 / 63(20.6 \%)$ specimens (Fig. 1B) with a mean count of $4.46 \pm 3.3$ T-lymphocytes/HPF $(x$ 320)(Table III). The mean count of all leucocytes in these 13 patients was $15.6 \pm 12.6$ (leucocytes/HPF), T-lymphocytes were of the suppressor/cytotoxic phenotype (leu-2a +) (Fig. 1C). Although occasionally leu- $3 a+$ cells (helper/inducer T-cells) were seen (Fig. 1D), no leu-12 + cells (B-lymphocytes) were detected in any of the specimens. In smears stained with Papanicolaou technique, leucocytes were reported only in $2 / 60(3.3 \%)$ patients as 2 leucocytes/HPF $(\times 100)$.

\section{Comment}

Monoclonal antibodies with specificity to human leucocyte surface markers were used in an indirect immunoperoxidase technique to stain cytocentrifuge smears of washed ejaculate. This
TABLE III. Lymphocytes in semen detected by anti leu-4 monoclonal antibody (a pan T-cell marker)

\begin{tabular}{ccc}
\hline \multirow{2}{*}{$\begin{array}{c}\text { Leucocytes/HPF } \\
(\times 320)\end{array}$} & Patients & $\begin{array}{c}\text { Number of- } \\
\text { Fertile Men } \\
\text { (Controls) }\end{array}$ \\
\hline 0 & 50 & 10 \\
$1-10$ & 12 & 0 \\
$11-20$ & 1 & 0 \\
\hline
\end{tabular}

immunocytochemical technique has permitted precise identification of leucocytes in the semen. This procedure which utilizes specific monoclonal antibody probes allowed further classification and characterization of the leucocytes into their subpopulations.

The technique has many advantages, mainly its high specificity. For example, the antihuman leucocyte monoclonal antibody (HLe-1) has been shown to react with $>95$ per cent of lymphocytes, monocytes, polymorphonuclear cells, and eosinophils in peripheral blood, spleen, and tonsil. ${ }^{13}$ Furthermore, in a study on frozen sections of human peripheral testis, we have reported that the antibodies used in this study do not react with the germinal epithelium, sperm precursors, or mature sperms. ${ }^{16}$ Therefore, with immunoperoxidase techniques the staining was very specific, and the danger of false negative and false positive results was circumvented by simultaneous study of known positive and negative controls.

It should be stressed that the use of diaminobenzidine/hydrogen peroxide substrate in this technique may allow visualization of the peroxidase positive granules, if still active, in the neutrophils, thus allowing the simultaneous exploration of currently used techniques to identify this cell population. It also should be noted that fixed smears may be examined simultaneously for morphology and staining by light microscopy. The stained preparations provide a permanent record which can be stored at room temperature, and the use of high dilutions of the monoclonal antibodies $(1 / 100)$ makes this technique relatively inexpensive.

In the present study PMN leucocytes were the predominant cell type of leucocytes in the ejaculate in both fertile and subfertile groups. Tlymphocytes were demonstrated only in 13/63 specimens from subfertile patients, and these lymphocytes represented 28.1 per cent of the total leucocyte count in these 13 specimens. This 
is in contrast to the findings of Couture $e t a l .^{7}$ In their study, lymphocytes predominated and represented 66.6 per cent of the total count of leucocytes, while PMN leucocytes represented only 33.3 per cent of the total count. A possible explanation of the difference in results is that in Couture's study round immature germ cells in which the acrosomal cap staining was deficient or difficult to visualize were counted as lymphocytes thus increasing their number and perhaps the PMN leucocytes which failed to show peroxidase-positive granules were considered to be non-separated spermatids, thus lowering the number of PMN leucocytes.

Previous reports have demonstrated immunosuppressive factors in human seminal plasma which can interfere, either directly or indirectly, with the function of most cells of the immune system including T-cells, B-cells, NK cells, and macrophages. ${ }^{17}$ The existence of such seminal immunosuppressive factors may explain the rarity of lymphocytes in the ejaculate of fertile men. Instead of having lymphocytes, which have specific and delayed action, the ejaculate is endowed with cells which are capable of performing immediate action.

Our finding that the fertile men had large numbers of PMN leucocytes compared with infertile men raises many questions about the normal role of these cells in the ejaculate. The primary role of PMN leucocytes is internalization and killing of microorganisms by the accumulation of toxic products within phagocytic vacuoles. However, extracellular release and cytotoxicity of these agents including reactive oxygen derivative and products of myeloperoxidase system are well documented. ${ }^{18}$ So the existence of PMN leucocytes in the ejaculate may provide a nonspecific and rapid defense mechanism and protection against invading organisms. The presence of these cells often is used as a criterion of subclinical genital infection but, perhaps, those cells have another role.

It is well known that during spermiogenesis, excess spermatid cytoplasm is shed as residual bodies. ${ }^{12}$ These cytoplasmic fragments are phagocytosed, broken down, and subsequently resorbed by Sertoli cells lysosomes. A small portion of cytoplasm, the cytoplasmic droplet, however, remains attached to the midpiece of spermatozoa. So, it is possible that the PMN leucocytes in the ejaculate have a role in the final phagocytosis of the residues of degenerating germ cells and of residual bodies in the ejaculate. On the other hand, subfertile men have a high percentage of abnormal sperm forms which would, perhaps, result in an overstimulation of phagocytosis but for the presence of suppressor/cytotoxic cells.

Our findings need to be confirmed by others, but they do cast doubt on the traditional criteria of subclinical genital tract infection.

\section{Edinburgh EH4 2XU, Scotland (DR. EL-DEMIRY)}

\section{References}

1. Comhaire F, Vershraegen G, and Vermeulen L: Diagnosis of accessory gland infection and its possible role in male infertility, Int J Androl 3: 32 (1980).

2. Morton RS: White cell counts in human semen. Their use in the diagnosis of prostatitis with reference to uveitis, $\mathrm{Br} \mathrm{J}$ Vener Dis 44: 72 (1968).

3. Johannisson E, and Eliasson R: Cytological studies of prostatic fluids from men with and without abnormal palpatory findings of the prostate, 1. Methodological aspects, Int J Androl 1: 201 (1978).

4. Holstein C: Morphology of free immature germ cells in human testis, epididymis, and ejaculate, Andrologia 15: 7 (1983).

5. Endtz AW: Ein methode om het vochtige urinesediment en het vochtige menselijke sperma rechtst reeks et kleuren, Ned Tijdschr Geneeskd 116: 681 (1972).

6. Nahoum CRD, and Cardozo D: Staining for volumetric count of leukocytes in semen and prostate-vesicular fluid, Fertil Steril 34: 68 (1980).

7. Couture M, Ulstein M, Leonard J, and Paulsen CA: Improved staining method for differentiating immature germ cells from white blood cells in human seminal fluid, Andrologia 8: 61 (1976).

8. Bryan JHD: An eosin-fast green naphthol yellow mixture for differential staining of cytologic components in mammalian spermatozoa, Stain Technol 45: 231 (1970).

9. Lillie RD: Histopathological technique and practical histochemistry, New York, McGraw-Hill Book Company, 1965, p 137.

10. Nelson DA, and Davey FR: Leukocyte peroxidase, in Williams WJ: Hematology, ed 2, chapt A20, New York, McGraw-Hill Book Company, 1977, p 1627.

11. Belsey MA, et al. (Eds): WHO Laboratory Manual for Examination of Human Semen and Semen Cervical Interactions, Geneva, World Health Organization, 1980, pp 18-21.

12. Clermont $Y$ : Dynamics of human spermatogenesis, in Rosenberg E, and Paulsen CA (Eds): The Human Testis, New York, Plenum Press, 1970, p 47.

13. Beverley PCL: Production and use of monoclonal antibodies in transplantation immunology, in Touraine JL, et al: Transplantation and Clinical Immunology XI, Amsterdam, Excerpta Medica, 1980, p 87.

14. Ledbetter JA, et al: Evolutionary conservation of surface molecules that distinguish T-lymphocyte helper/inducer and T-cytotoxic/suppressor subpopulations in mouse and man, J Exp Med 153: 310 (1981).

15. Engleman EG, Benike CJ, Glickman E, and Evans RL: Antibodies to membrane structures that distinguish suppressor/ cytotoxic and helper T-lymphocytes subpopulations block the mixed leucocyte reaction in man, ibid 154: 193 (1981).

16. El-Demiry MIM, et al: Lymphocyte subpopulations in the male genital tract, Br J Urol 57: 769 (1985).

17. James $\mathrm{K}$, and Hargreave $\mathrm{TB}$ : Immunosuppression by seminal plasma and its possible clinical significance, Immunol Today 5: 357 (1984).

18. Clark RA: Extracellular effects of the myeloperoxidase hydrogen peroxide halide system, in Weissman GL (Ed): Advances in Inflammatory Research, vol 5, New York, Raven Press, 1983, p 107. 


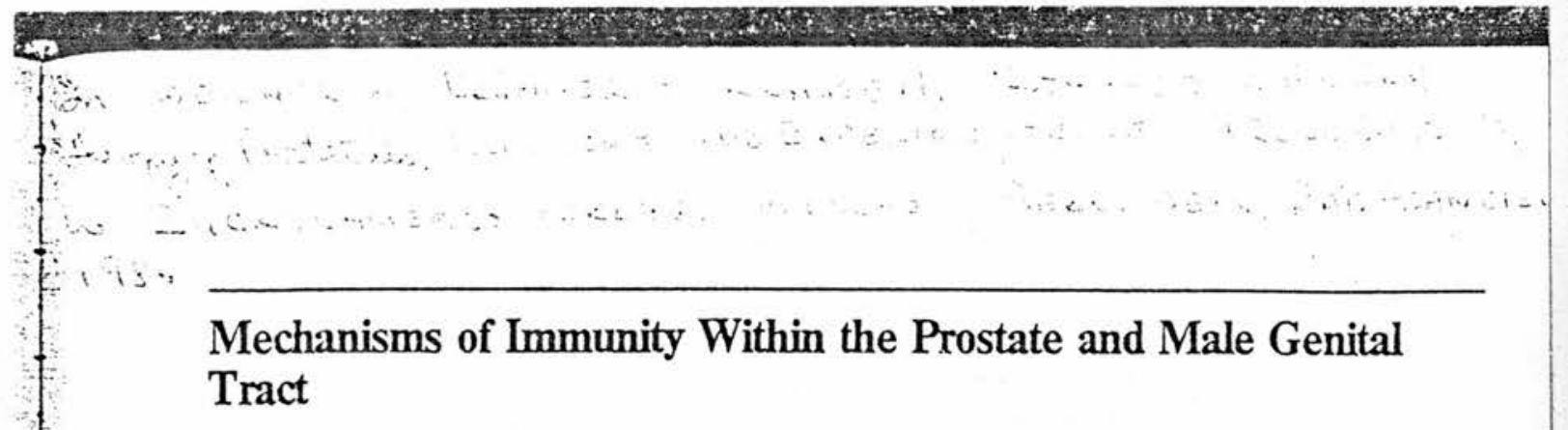

\author{
T.B. Hargreave, K. James, G.D. Chisholm, M. El Demiry, S. Szymaniec, J. Harvey \\ and A.W.S. Ritchie \\ Infertility clinic, Western General Hospital, University of Edinburgh, Scotland, United \\ Kingdom
}

\section{Introduction}

The male genital tract is an area of considerable immunological interest because there is a conflict of need. Antigenic sperm first appears at an age when the immune system is fuily developed and therefore not recognised as "self $"$. When injected they will produce an autoimmune reaction. The first need is to shield sperm from the immune system both in the male and female genital tracts. However, the male urethra is one of the potential pathways of entry of bacterial organisms into the body. Also the close proximity of the urethra to the rectum means that there is a high level of contamination. The second need therefore is for an effective defence against organisms ascending the urinary and genital tract. In our department we have for.the past five years been investigating genital tract immunity mechanisms which may be relevant to how this balance is achieved. The purpose of this paper is to summarise our work with specific references to prostatitis.

\section{Patients}

Serum and semen samples were obtained during the course of routine investigation of male patients attending the infertility clinic at our hospital. Testicular and epididymal biopsy samples were obtained during the course of investigation of infertility. Prostatic biopsy specimens were obtained from prostatecto- my specimens following operations for benign prostatic hypertrophy. Normal maie genital tract tissue including prostatic tissue was obtained from young men who had been killed accidentally and where relatives had given permission for multi organ donation for transplantation and research.

\section{Methods}

The methods used have all been described fully elsewhere $(3,0)$. The following techniques were used to study the effect of seminal plasma:

1) the effect of concanavalin A induced transformation of mouse and human lymphocytes; 2) effect on concanavalin $A$ induced transformation of mouse lymphocyte after depletion of adherent accessory cells and reconstitution with peritoneal exudate cells from seminal plasma treated mice; 3 ) the effect on the ability of $C$. parvum treated mouse peritoneal exudate cells to attach to glass, spread and phagocytose fluoroscein coated latex beads; 4) the effect on the chemiluminescence response of human peripheral blood monocytes to opsonised zymozan; 5) the effect on chemiluminescence response of human neutrophils to opsonised zymozan; 6 ) the effect on the expression of $F_{c}$ (but not $C 3$ ) receptors by macrophages.

More recently we have also examined the effect of seminal plasma on the natural killer 
(NK) activity of peripheral blood ?eucocytes (4).

For the immunohistologic studies frozen sections were cut using a cryostat and staining

Table I. Summary of our findings of the inhibitory effects of seminal plasma on cells of the immune response (3).

\begin{tabular}{ll}
\hline Target & Response suppressed \\
\hline $\begin{array}{l}\text { T lymphocytes } \\
\text { human and murine }\end{array}$ & $\begin{array}{l}\text { Proliferative response to } \\
\text { concanavalin A }\end{array}$ \\
$\begin{array}{l}\text { Monocytes and } \\
\text { macrophages }\end{array}$ & $\begin{array}{l}\text { Attachments and spreading } \\
\text { of activated macrophages } \\
\text { Expression of Fc (but not } \\
\text { C3 receptors }\end{array}$ \\
& $\begin{array}{l}\text { Phagocytosis of latex parti- } \\
\text { cles } \\
\text { Generation of reactive oxy- } \\
\text { gen species } \\
\text { Lysis of susceptible target } \\
\text { cells (4) } \\
\text { Generation of reactive oxy- } \\
\text { gen species }\end{array}$ \\
Natural killer cells & \\
Neutrophils & \\
\hline a Szymaniec and James (unpublished data)
\end{tabular}

was undertaken using a panel of monoclanal antibodies against $\mathrm{T}$-cells and their sub-sets. The antisera used were purchased from Coulter-Electronics, Bedforshire, England. They included reagents reacting with all $T$ cells $\left(T_{11}\right)$, the suppressor/cytotoxic subset $\left(T_{8}\right)$ and the helper/inducer subset $\left(T_{4}\right)$. The slides were then stained using immunoperoxidase and counterstained with haematoxilin. The full details of the method have been published elsewhere $(6,1)$.

\section{Results}

Our seminal plasma results which are summarised in table 1 confirm and extend the work of others. Seminal plasma has a potent immunosuppressive effect and has been demonstrated by us and others to suppress $T$ lymphocytes, B-lymphocytes, NK-cells, macrophages and neutrophils (2). The variety of effects observed might be due to different fractions within seminal plasma (3) but it has so far not been possible to determine whether

Table II. Summary of our findings from immunohistological studies of normal tissue from the male genital tract (data from $(6,1)$.

\begin{tabular}{|c|c|c|}
\hline \multirow[t]{2}{*}{ Tissue } & \multicolumn{2}{|l|}{ Finding } \\
\hline & intraepithelial & interstitial tissues and lamina propria \\
\hline Testis & & \\
\hline peripheral & None & Occasional T helper/inducer cells \\
\hline rete & $\begin{array}{l}\text { Occasional T suppressor/ } \\
\text { cytotoxic }\end{array}$ & $\begin{array}{l}\text { Occasional T helper/inducer cells } \\
\text { in the interstitium }\end{array}$ \\
\hline Epididymis & $\begin{array}{l}\text { T suppressor/cytotoxic cells } \\
\text { throughout the epithelium of } \\
\text { the epididymis }\end{array}$ & $\begin{array}{l}\text { Aggregates of } \mathrm{T} \text { helper/inducer ceils } \\
\text { in the interstitium }\end{array}$ \\
\hline Vas & $\begin{array}{l}\text { Dense population of intraepi- } \\
\text { thelial T suppressor/cytotoxic } \\
\text { cells }\end{array}$ & Occassional T helper/inducer \\
\hline $\begin{array}{l}\text { Seminal vesicle and prostate } \\
\text { (figure 1) }\end{array}$ & $\begin{array}{l}\text { Suppressor/cytotoxic cells } \\
\text { within the epithelium though } \\
\text { less prevalent than in the } \\
\text { epididymis }\end{array}$ & $\begin{array}{l}\text { Occasional aggregates of } \mathrm{T} \text { helper/ } \\
\text { inducer cells }\end{array}$ \\
\hline
\end{tabular}


the components responsible are secreted by the prostate, seminal vesicles or epididymis. Our tissue results demonstrate that in addition there may be a mechanism of mucosal immunity within the genital tract. The results are summarised in table II. We find a common pattern throughout the genital tract. $T$ cells of the suppressor cytotoxic subset are present within the epithelium while the majority of the T-cells of the helper/inducer subset are found within the interstitium. This pattern is also true for the prostate gland.

\section{Discussion}

Our studies demonstrate two possible mechanisms for moduiation of the immune response within the genital tract. We confirm the work of Prakash and Lang (1980) and others reviewed in James and Hargreave (1984) that seminal plasma has strong immunosuppressive effects. Our studies (3) demonstrate a particular suppression of macrophage function which is relevant as macrophages are thought to "programme" both cellular and humoral immune responses. The components of seminal plasma responsible for immunosuppression have not yet been characterised but more than one fraction seems to be responsible. At present we are working to characterise the components. Once we have done this we hope to find which of the accessory sex glands is responsible for their production by examination of split ejaculate samples and further immunological studies. If some or all of these components arise in the prostate gland this may help explain why prostatitis is such a chronic condition.

Our immunohistological studies demonstrate another possible reason for the chronicity of the genital tract infections. We find a predominance of T-cells of the suppressor/ cytotoxic subset within the epithelium. This finding has now been confirmed by two studies from our department $(6,1)$ and as far as we

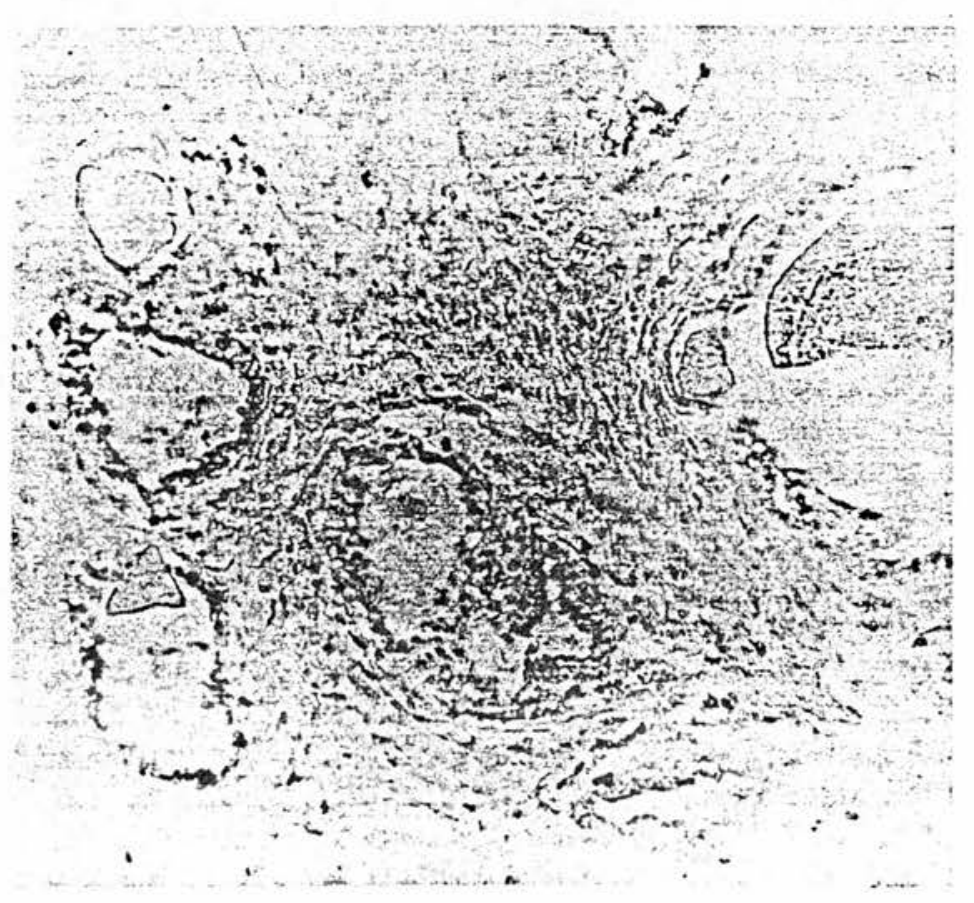

Figure 1. Section of normal prostate stained with McAB for T suppressor/ cytotoxic cells. Note the suppressor/cytotoxic cells appear as black in this black and white photograph and are mainly in the epithelium. The distinction is much clearer in colour as the $T$ cells appear brown against a blue background. For colour illustrations see (6). 
know this is the first time these observations have been made within the genital tract. The pattern we find within the genital tract is however similar to that seen in human intestinal mucosa (7) where the high suppressor cell content may inhibit the response to dietary antigen. As we have suggested it is possible that the presence of T-suppressor/cytotoxic cells within the epithelium of the epididymis, vas and prostate may minimize sensitisation to autoantigens of the sperm. One consequence of this might be an impaired response to opportunistic microorganisms. We do not yet know whether the cells within the genital tract mucosa are suppressor or cytotoxic because monoclonal antibodies to further differentiate the cell types were not available to us when we did our studies. Such antibodies are now being developed and we hope soon to be able to define whether the mucosal cells are suppressor or cytotoxic. We also hope to try to collect sufficient numbers of cells to untertake functional studies to see if the cells are active or not.

Clinicians have been aware for many years that prostatitis is a difficult disease to treat and often recurrs. There has however been no satisfactory explanation why this should be so. The clinical situation has been further confused because prostatitis is difficult to diagnose as the symptoms are nonspecific and often there is no bacteriological evidence. In a proportion of cases the symptoms suggestive of prostatitis may in fact be caused by bladder neck obstruction and urodynamic evaluation provides the answer. Nevertheless when histological specimens are examined or when more accurate culture techniques are used, for example, the four-specimen-technique (8) a population of men can be defined who have proven prostatitis with microbiological evidence. Often, however, despite long term therapy with tetracycline, an antibiotic which is known to be toxic to the organisms concerned, the condition relapses. We suggest that the genital tract immunosuppressive mechanisms we have de- scribed in this paper may be relevant to this problem of relapse. These mechanisms may also be relevant to other infections within the genital tract and to the acquired immune deficiency syndrome (AIDS) and finally to cancer of the cervix. It is our hope and at present it can only be a hope, that better understanding of these mechanisms may lead to improved understanding of genital tract disease and to improvements in therapy.

\section{References}

1 El Demiry MIM, Hargreave TB, Busuttil A, James K, Ritchie AWS, Chisholm GD (1985) Lymphocyte subpopulations in the male genital tract. Br J Urol 57: 769

2 James K, Hargreave TB (1984) Immunosuppression by seminal plasma and its possible clinical significance. Immunol Today 5: 357

3 James K, Harvey J, Bradbury AW, Hargreave TB, Cullen RT, Donaldson K (1983) Preliminary report: The effect of seminal plasma on macrophage function - a possible contributory factor in sexually transmitted disease. Aids Res 1: 45

4 James K, Szymaniec S (in press) Human seminal plasma is a potent inhibitor of natural killer cell activity in vitro. J reprod Immunol

5 Prakash C, Lang RW (1980 Studies on immune fertility: A hypothesis on the etiology of immune fertility based on the biological role of seminal plasma immune response inhibitor. Mt Sinai J Med 47: 491

6 Ritchie AWS, Hargreave TB, James K, Chisholm GD (1984) Intra-epithelial lymphocytes in the normal epididymis. A mechanism for tolerance to sperm auto-antigens? $\mathrm{Br} \mathrm{J}$ Urol 56: 79

7 Selby WS, Janossy G, Goldstein G, Jewell JP (1981) T-lymphocyte subsets in human intestinal mucosa: the distribution and relationship to MHC-derived antigens. Clin exp Immunol 44: 453

8 Weidner W, Krause W, Schiefer HG, Brunner H, Friedrich HJ (1985) Ureaplasma infections of the male urogenital tract, in particular prostatitis, and semen quality. Urol Int 40: 5

Corresponding address: T. B. Hargreave, M. D., Infertility clinic, Western General Hospital, Edinburgh, EH4 2XU, Scotland 


\title{
The Role of Ureaplasma Urealyticum in Male Infertility
}

\author{
T.B. Hargreave, H. Young ${ }^{a}$, I.W. Wood ${ }^{a}$, J. Harvey, M. El Demiry, K. James and \\ G.D. Chisholm \\ Department of Surgery/Urology, Western General Hospital, and \\ 'Department of Bacteriology, Medical School, Teviot Place, University of Edinburgh, \\ Edinburgh, United Kingdom
}

\section{Introduction}

There is conflicting information in the literature about the role in male infertility of subclinical genital tract infection (10) and in particular infection with Ureaplasma urealyticum (22). It is therefore relevant to ask why there is difficulty in obtaining clear evidence. There are several good reasons for the difficulties and some are common to all attempts to assess the causes of male infertility. The first problem is that it is very difficult to define what is male fertility for the obvious reason that the end result, a successful pregnancy, involves the female partner and therefore introduces another set of variables. This would not matter if there was good or exact correlation between semen analysis measurements and fertility, but there is not (11). Indeed the standard techniques of semen analysis (23) are inaccurate (17). The use of accurate clinical record keeping for both male and female partner and multivariate methods of statistical analysis (12) may give better help in the future when relating male laboratory results to male fertility but the numbers of variables mean that computer facilities have to be used. So far this approach has not been used to analyse the results of semen bacterial culture but it has only been possible to use less sophisticated techniques such as the comparison of results between a population of men complaining of an infertile marriage compared with men with a fertile marriage or simply to relate culture results from men with an infertile mar- riage to symptoms or other laboratory measurements. However these methods of assessing the role of infection, provide only indirect evidence about fertility. When considering Ureaplasma urealyticum there is an additional problem that the organism is difficult to culture, some serotypes may be pathogenic but others only commensual and facilities for quantitative culture do not exist in all hospitals. We are fortunate in this city to have good facilities for bacterial culture as well as a busy infertility clinic and we have taken the opportunity to investigate the role of Ureaplasma infection. The purpose of this paper is to review our work to date to help answer the question does subclinical Ureaplasma urealyticum infection play any part in male infertility? We have unfortunately only been able to do simple studies involving the correlation of Ureaplasma culture results with other laboratory measurements and for the reasons given above our results cannot give the final answer but rather they provide some more evidence. It is relevant to note that our results relate only to patients complaining of an infertile marriage and who did not have any symptoms or signs of prostatitis or genital tract infection i.e. true subclinical infection.

\section{Patients}

Our first study was performed in 1981 (13) and the second started in 1985 and is continuing. Semen samples were collected from patients 
attending a general infertility clinic. The-policy in the clinic is to fully investigate and offer treatment to both husband and wife as well as conduct studies into male infertility. The purpose of our first study was to search for correlation between antisperm antibodies and Ureaplasma culture to try to define whether such an infection may have a role in triggering antibody production. Because the study objective was to study cause of antisperm antibody production the clinic appointments were biased during the study period to give an increased number of attenders who were known to have antisperm antibodies from previous tests. It was possible although extremely unlikely that this bias had inadvertently excluded patients with significant Ureaplasma infection and it was therefore decided to conduct a second study and this was started in 1985. In order to eliminate any inadvertent bias in our second study semen samples were cultured for Ureaplasma from consecutive new patients attending the clinic.

\section{Methods}

Semen samples were collected and immediately taken to the laboratory. Using sterile precautions an aliquot was extracted for standard perm measurements. The remainder of the specimen was transported to the bacteriology department for culture. The methods of semen analysis were standard as described in the World Health Organization laboratory manual (23).

\section{Antisperm antibody measurement}

The methods have been described elsewhere (13). The Tray agglutination test (TAT) (9) and the microimmobilisation test (MIT) (15) were used.

\section{Ureaplasma urealyticum culture}

The methods have been described elsewhere (13). Seminal plasma $(0.2 \mathrm{ml})$ was added to 1.8 ml of TALC (Trimethoprim, amphotericin, lincomycin, colistin) colour change medium (24) and transported to the laboratory within four hours of collection. On receipt in the laboratory a series of tenfold dilutions was made in $1.8 \mathrm{ml}$ volumes of TALC medium: the final dilution was $10^{10}$. Cultures were incubated at $37^{\circ} \mathrm{C}$ and examined after 24 and 48 hours. The presence of Ureaplasmas was indicated by a colour change in the medium from yellow to pink. One colour-changing unit (CCU) of activity was defined as the highest dilution of the Ureaplasma suspension that produced a colour change (20).

\section{Seminal teucocyte staining}

Seminal leucocytes were estimated in two ways. The standard method was to make a direct smear from the sperm sample and to stain it using the Papanicolaou method. The number of white cells per high power field (times 100) were then estimated.

There is, however, some doubt about the accuracy of discrimination of leucocytes from immature sperm forms when the Papanicolaou method is used and for this reason there has been a search for more specific staining methods (3). During our second study period we have used a new sensitive monoclonal antibody based immunoperoxidase procedure (๑). The monoclonal antibody used was directed against all leucocytes but did not stain sperm or sperm precursors. Because of the expense and limited quantity of reagents we stained cytospin preparations of washed ejaculate. Varying dilutions of the washed and resuspended ejaculate were made to given approximately constant sperm numbers $\left(10^{6}\right.$ per $\left.\mathrm{ml}\right)$ to make counting practical. Leucocyte counts were then made for five high power fields and the average taken. In order to minimise mathematical manipulation of the readings we have chosen in this paper not to report the leucocyte count in absolute terms but simply to multiply the count per high power field by the dilution. 
The resulting numbers of leucocytes reported in the tables do not have any independent meaning but they do allow comparison between patients and distinction of those with high or low leucocyte numbers. Further work is in progress when the same technique will be applied to samples from a fertile control group and the results will be correlated with culture results for a variety of anaerobic and aerobic organisms (7). The full work will be published later but the preliminary results which pertained to Ureaplasma culture are presented here.

\section{Results}

In our first study (13) sperm samples were cultured for Ureaplasma from 115 men and correlations were made between the culture results, sperm measurements and antisperm antibody measurements. The results are summarised in table I which shows that there was no correlation between Ureaplasma culture results and any of the other test results.

Table I. Summary of results from first Ureaplasma study (13).

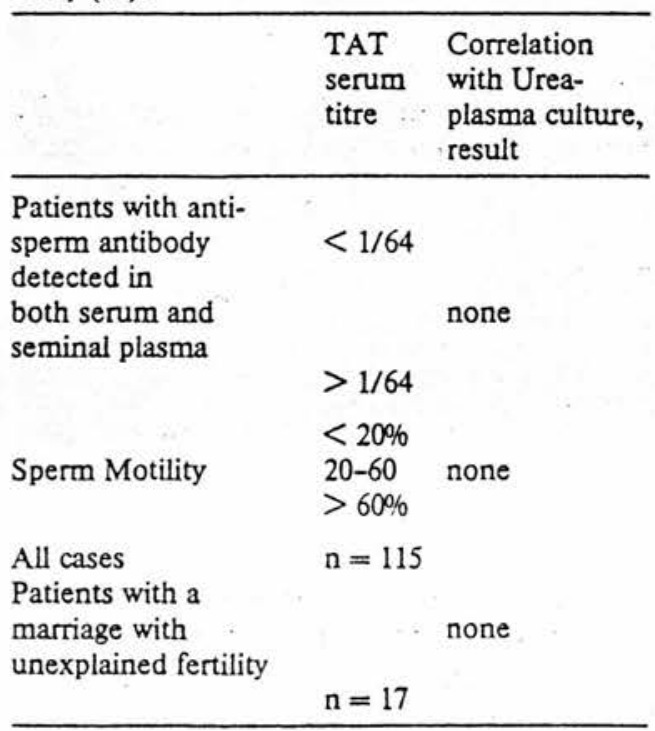

Table II. Correlation between Ureaplasma culture and sperm motility - 2nd study period.

\begin{tabular}{llc}
\hline & \multicolumn{2}{c}{ Ureaplasma culture } \\
\cline { 2 - 3 } & +ve & -ve \\
\hline Progressive motility & & \\
$<10 \%$ & 1 & 5 \\
$11-60 \%$ & 3 & 14 \\
$>60 \%$ & 4 & 24 \\
$\mathrm{X}^{2}=0.10,2$ d.f., NS & & \\
\hline
\end{tabular}

Table III. Correlation between Ureaplasma culture and leucocyte count (Papanicolaou method) - 2nd study period.

\begin{tabular}{llc}
\hline & \multicolumn{2}{l}{ Ureaplasma culture } \\
\hline Leucocytes, Pap. stain & +ve & - ve \\
Present & & 3 \\
Absent & 8 & 40 \\
$\mathrm{X}^{2}=0.53$ 1 d.f., NS. & & \\
\hline
\end{tabular}

The possibility remained however that in some cases the Ureaplasmas were commensual but in others pathogenic and the problem was how to differentiate between these two alternatives. We decided to repeat a study of Ureaplasma culture results in samples from consecutive new patients attending our clinic. The results from the first 55 patients are shown in the tables. Four patients did not have either Papanicolaou stain or progressive motility measurement and this accounts for only 51 patients appearing in tables II and III. In table II the Ureaplasma results are shown in relation to sperm motility and again no correlation was seen. This supports our first study and is also in agreement with the findings of Weidner et al. (1985). The results of correlation between leucocyte counting using the Papanicolaou staining method and Ureaplasma culture is shown in table III. There was again no correlation although only eight patients had leucocytes detected using this method and the highest count was 3 per high power field. It was because of the doubts about accuracy of this method that a new spe- 
Table IV. Correlation between Ureaplasma culture and leucocyte count (McAB stain) - 2nd study 55 men with an infertile marriage.

\begin{tabular}{|c|c|c|}
\hline \multirow{3}{*}{$\begin{array}{l}\text { No of } \\
\text { leucocytes } \\
\text { per field }\end{array}$} & \multicolumn{2}{|c|}{ Ureaplasma culture } \\
\hline & $+\mathrm{ve}$ & $-\mathrm{ve}$ \\
\hline & $\begin{array}{l}\text { patients, } n \\
(\% \text { of }+v e)\end{array}$ & $\begin{array}{l}\text { patients, } n \\
(\% \text { of }-v e)\end{array}$ \\
\hline $\begin{array}{l}<1 \\
1-20 \\
>20 \\
X^{2}=0.232 \text { d.f., NS }\end{array}$ & $\begin{array}{l}1(12) \\
5(62) \\
2(24)\end{array}$ & $\begin{array}{r}9(19) \\
26(56) \\
11(24)\end{array}$ \\
\hline
\end{tabular}

cific staining technique for leucocytes was devised. The results of the correlation between leucocyte staining using the monoclonal antibody method and Ureaplasma culture are shown in table IV and despite leucocytes being detected in all but five patients there was again no correlation between the two sets of measurements.

\section{Discussion}

There is conflicting evidence about the role of any infection in male infertility. The first distinction to be made is between symptomatic clinically overt infection such as sexually transmitted disease (STD), prostatitis, epididymitis, orchitis, etc and sub-clinical infection.

There is a well known association between STD and infertility usually secondary to the complications rather than the primary infection. The most common complications causing infertility are epididymitis with secondary epididymal obstruction and urethral stricture especially if the later requires surgical repair. The percentage of cases of male infertility secondary to STD is very variable depending on local customs and the availability of effective medical help. In the United Kingdom clinics for sexually transmitted disease are generally available and most men with urethritis seek help rapidly. Despite the high prevalence of STD in the UK (2) treatment is usually rapid and its complications do not seem to be a common cause of male infertility in our clinic. It could be argued that many of our patients have not told us about their past history of STD. Evidence to back up our impression of the minor role of STD induced male infertility in our clinic can be found by examining our clinic records of men with obstructive azoospermia. From the last two hundred men with obstructive azoospermia we have identified only one such case. Our most common cause of obstructive azoospermia was congenital obstruction probably due to a failure of fusion of ducts between the head and body of the epididymis. The clinical finding is an engorged epididymal head with an empty epididymal body and tail and with no evidence of any inflammatory damage of adhesions. Our second most common cause is Young's syndrome (azoospermia in association with respiratory disease). Even cases of azoospermia secondary to previous surgical trauma are more common in our practice than those after venereal disease. This low incidence of azoospermia secondary to gonococcal epididymitis is completely different to that reported in some other series (19). It is probably reasonable to assume that the low incidence of STD induced male infertility we find in our azoospermic patients is also true for the rest of our patients. In the last two hundred unselected patients we have seen only ten gave past history of STD although all were asked.

If overt STD does not play a major role in our male patients could subclinical genital tract infection be important? The majority of men attending our clinic have oligozoospermia but there is no obvious cause. There is conflicting evidence in the literature about the role of infection in general (10) and Ureaplasma urealyticum infection in particular. De Louvois et al. (1974) found no difference in the frequency of Ureaplasma urealyticum between a fertile and infertile group of patients but Idriss et al. (1978) found that $44 \%$ of pa- 
tients with unexplained infertility had Ureaplasma urealyticum infection against $32 \%$ in a control population and this difference was significant. Ureaplasma urealyticum has been shown to stick to sperm (8) and therefore because of the conflicting reports in the literature we thought it worthy of further study. In our first study of 115 men we found no obvious correlation between Ureaplasma culture and the fertility status of the patient, sperm motility or the presence of antisperm antibodies. The isolation of Ureaplasma from $51(44.3 \%)$ of 115 men in our first study is similar to the incidence $32.6 \%$ reported previously in a similar population (5). These isolation rates are also similar to those obtained in other populations; when urine specimens from men with non gonococcal urethritis and from men with no urethritis were cultured, Ureaplasma was found in 53\% and $33 \%$ of patients respectively (14). These findings lend support to the suggestion of Desai et al. 1980 that the presence of Ureaplas$\mathrm{ma}$ in the semen is merely the result of contamination at the time of ejaculation. We could not, however, entirely exclude the possiblity that in some cases Ureaplasma were commensual but in others pathogenic. The problem remained to distinguish the mere presence of Ureaplasma in semen samples as a result of contamination during passage through the urethra from their aetiological role in fertility. One possible index of the pathogenecity of organisms in the semen is the leucocyte response and we therefore decided to repeat the Ureaplasma culture work in association with a special attempt to distinguish leucocytes from immature sperm cells. Because of doubt about our ability to distinguish leucocytes from immature sperm forms using Papanicolaou method we have also used a specific leucocyte monoclonal antibody ( $\mathrm{Pan} \mathrm{T}$ cells) in association with the immunoperoxidase technique. Despite the use of this more sophisticated and sensitive technique our preliminary results show no correlation between Ureaplasma culture results and leucocyte response. This result may be interpreted in a number of ways. Firstly the number of patients in our preliminary report is small and we may still be missing the occasional case where Ureaplasma is in fact pathogenic. It should be remembered that the patients we are studying are asymptomatic presenting with infertility and this may explain why our results differ in some respects from those published by Weidner et al. (1985) where the population under study was 597 men with symptoms of chronic prostatitis attending a special prostatitis clinic. Our results are in agreement with those published by Upadhaga et al. (1984) who also studied an asymptomatic population on men. Another possible interpretation of our results is that other organisms may be responsible. Several of our patients in fact had large numbers of leucocytes and although we find no correlation with Ureaplasma culture results there may be correlation with culture results for other organisms. We are currently expanding our series and performing a full range of cultures and hope to be able to comment about this second possibility in the future.

Finally we did not serotype the Ureaplasma and the possibility remains that we missed infection with those serotypes which are thought to be significant. With this in mind it is interesting to note that Busalo and Zanchel$\operatorname{ter}(1985)$ found Ureaplasma urealyticum serotypes 4,8 and 6 had a high infection activity with in vitro penetration by human sperm of hamster eggs.

In conclusion the evidence from both our Ureaplasma studies suggests that this organism does not play a major role in male infertility if the patient has no symptoms of prostatitis. We did not find any correlation between the fertility status of the patient, sperm motility, the presence of antisperm antibodies and the leucocyte response in the semen. We have therefore found no evidence for true subclinical Ureaplasma urealyticum infection as a major factor in causing male infertility. 


\section{References}

1 Busolo F, Zanchetta R (1985) The effect of Mycoplasma hominis and Ureaplasma urealyticum on hamster egg in vitro penetration by human spermatozoa. Fertil Steril 43: 110

2 Communicable Diseases Surveillance Unit, Middlesex Hospital Medical School, Communicable Diseases (Scotland) (1979) Sexually transmitted disease: surveillance 1978. Br Med J ii: 1375

3 Couture M, Ulstein M, Leonard J, Paulsen CA (1976) Improved staining method for differentiating immature germ ceils from white blood cells in human seminal fluid. Andrologia 8: 61

4 de Louvois J, Blades M, Harrison RF, Hurley R, Stanley VC (1974) Frequency of mycoplasma in fertile and infertile couples. Lancet $\mathrm{i}: 1073$

5 Desai S, Cohen MS, Kharamee M, Leiter E (1980) Ureaplasma urealyticum (T-mycoplasma) infection: does it have a role in male infertility? J Urol 124: 469

6 El Demiry J, James $\mathrm{K}$, Hargreave TB (in press 1985a) A new method of semen leucocyte estimation. Brit J Urol

7 El Demiry M, Young H, James K, Hargreave TB (in preparation 1985b) Bacteriological studies and semen leucocytes estimation in fertile and infertile men

8 Fowlkes DM, Dooher GB, O'Leary WM (1975) Evidence by scanning electron microscopy for an association between spermatozoa and T-mycoplasmas in men of infertile marriage. Fertil Steril 26: 1203

9 Friberg J (1974) A simple and sensitive micromethod for demonstration of sperm-agglutinating activity in serum from infertile men and women. Acta obstet gynec scand (suppl) 36 : 21

10 Hargreave TB (1983) Non-specific treatment to improve fertility. In: Hargreave TB (ed) Male Infertility. Springer, Berlin Heidelberg New York Tokyo, pp 240

11 Hargreave TB, Elton RA (1983) Is conventional sperm analysis of any use? $\mathrm{Br} \mathrm{J}$ Urol 55 : 774

12 Hargreave TB, McGowan B, Harvey J, Mc Parland $\mathrm{M}$, Elton RA (in press) Is a male infertility clinic of any use? $\mathrm{Br} \mathrm{J}$ Urol

13 Hargreave TB, Torrance M, Young H, Harris $A B$ (1982) Isolation of Ureaplasma urealyticum from seminal plasma in relation to sperm antibody levels and sperm motility. Andrologia 14: 223

14 Hunter JM, Smith IM, Peutherer JF, Macaulay A, Tuach S, Young $H$ (1981) Chlamydia trachomatis and Ureaplasma urealyticum in men attending a sexually transmitted diseases clinic. $\mathrm{Br} \mathrm{J}$ vener $\mathrm{Dis} 57$ : 130

15 Husted S, Hjort T (1976) Quoted in: Techniques for detection of iso- and auto-antibodies to human spermatozoa. Clin exp Immunol 23: 175

16 Idriss WM, Patton WD, Taymor ML (1978) On the etiologic role of Ureaplasma urealyticum (T-mycoplasma) infection in infertility. Fertil Steril 30: 293

17 Jequier AM, Ukombe EB (1983) Errors inherent in the performace of a routine semen analysis. Br J Urol 55: 434

18 Prakash C, Lang RW (1980) Studies on immune infertility: A hypothesis on the etiology of immune infertility based on biological role of seminal plasma immune response inhibitor. Mt Sinai J Med 47: 491

19 Schoysman R (1981) Presentation at 2nd Int Congr Andrology, Tel Aviv

20 Taylor-Robinson D, Martin-Bourgon C, Watanabe T, Addey JP (1971) Isolation of T-mycopiasmas from dogs and squirrel monkeys: biological and serological comparison with those isolated from man and cattle. J gen Microbiol 68: 97

21 Upadhyaya M, Hibbard BM, Walker SM (1984) The effect of urealyticum on semen characteristics. Fertil Steril 41: 304

22 Weidner W, Krause W, Schiefer HG, Brunner $\mathrm{H}$, Friedrich HJ (1985) Ureaplasmal infections of the male urogenital tract, in particular prostatitis, and semen quality. Urol int 40: 5

23 WHO Laboratory Manual for the examination of human semen and semen cervical interactions (1980) In: Belsey MA, Eliasson R, Gallegos AJ, Morghissi KS, Paulsen CA, Praval MRN (eds) Distributed by Special Programme Research Development and Research Training in Human Reproduction. World Health Organization, Geneva

24 Young H, Tuach S, Bain SR (1981) Incidence of Ureaplasma urealyticum in women attending a clinical for sexually transmitted disease. $\mathrm{J}$ infect Dis 3: 258

Corresponding address: $T$. B. Hargreave, $M . D$., Infertility Clinic, Western General Hospital, Crewe Road, GB-Edinburgh EH4 2XU 